Supporting Information

\title{
Visible Light Promoted Synthesis of $\alpha-\mathrm{CF}_{2} \mathrm{H}-$ Substituted Ketones by Radical Difluoromethylation of Enol Acetates
}

Zengqiang Feng, ${ }^{\dagger \mid}$ Baoxiang Zhu, ${ }^{\dagger \|}$ Bingbing Dong, ${ }^{\dagger}$ Li Cheng, ${ }^{\dagger}$ Yunpu Li, ${ }^{\dagger}$ Zechao Wang, * and Junliang $\mathrm{Wu}^{\dagger *}$

${ }^{\dagger}$ College of Chemistry, and Institute of Green Catalysis; Zhengzhou University, Zhengzhou 450001, P. R. China

${ }^{\ddagger}$ Division of Molecular Catalysis \& Synthesis, Henan Institute of Advanced Technology, Zhengzhou University, Zhengzhou 450001, P. R. China

Email:wujl@zzu.edu.cn

Email: zechaowang@zzu.edu.cn 


\section{Table of Contents}

1. General Information ......................................................................................................................................

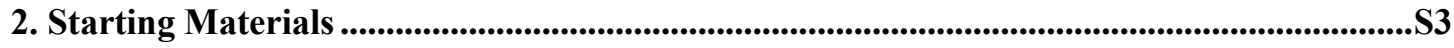

2.1 General Procedure for Synthesis of Enol Acetates ................................................................S3

2.2 General Procedure for Synthesis of $\mathrm{CF}_{2} \mathrm{H}$ Reagent 2a .....................................................S3

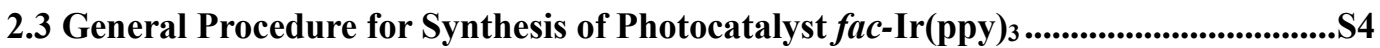

3. Detailed Optimization of Reaction Conditions................................................................................S5

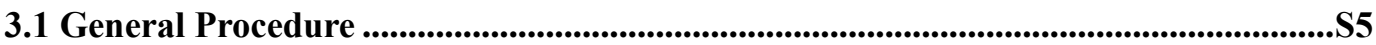

4. Investigation of Reaction Mechanism .....................................................................................................S8

4.1 BHT Trapping Experiment …............................................................................................58

4.2 TEMPO Trapping Experiment ........................................................................................................59

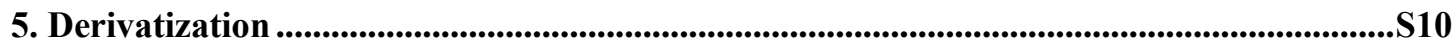

5.1 Synthesis of 3,3-difluoro-1-(p-tolyl)propan-1-ol (4) ............................................................S10

5.2 Synthesis of 4,4-difluoro-2-(p-tolyl)-2-((trimethylsilyl)oxy)butanenitrile (5)............S10

5.3 Synthesis of 4,4-difluoro-2-(p-tolyl)-2-((trimethylsilyl)oxy)butanamide (6) ..............S10

5.4 Reaction of the $\alpha-\mathrm{CF}_{2} \mathrm{H}$ ketone with Aniline...................................................................S11

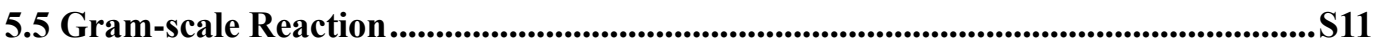

5.6 1-Phenylvinyl Trifluoromethanesulfonate as a Substrate...............................................S11

5.7 Trimethyl((1-phenylvinyl)oxy)silane as a Substrate........................................................S12

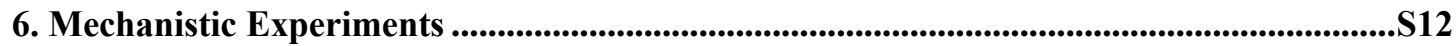

7. Stern-Volmer Quenching Experiments ..........................................................................................S14

8. X-ray Crystallographic Data of 3b .................................................................................................S15

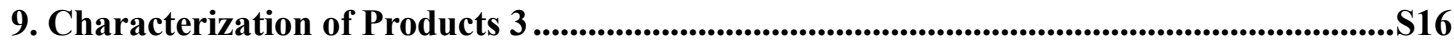

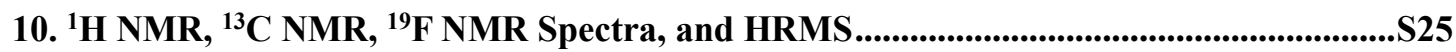

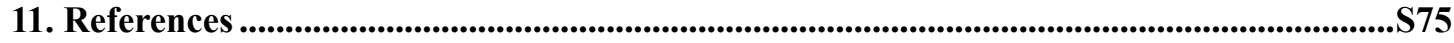




\section{General Information}

Unless otherwise noted, materials were purchased from commercial suppliers and used without further purification. All reactions were carried out in sealed tubes filled with argon. All reactions were monitored by TLC (thin layer chromatography) and visualized using UV light. Products were purified by column chromatography by 200-300 mesh silica. ${ }^{1} \mathrm{H}$ NMR spectra were recorded on 400 or 600 $\mathrm{MHz}$ spectrophotometers. Chemical shifts $(\delta)$ were reported in ppm from the resonance of tetramethyl silane as the internal standard (TMS: $0.00 \mathrm{ppm}$ ). Data were reported as follows: chemical shift, multiplicity $(\mathrm{s}=$ singlet, $\mathrm{d}=$ doublet, $\mathrm{t}=$ triplet, $\mathrm{q}=$ quartet, $\mathrm{m}=$ multiplet $)$, coupling constants $(\mathrm{Hz})$ and integration. ${ }^{13} \mathrm{C}$ NMR spectra were recorded on 100 or 151 with complete proton decoupling spectrophotometers. ${ }^{19} \mathrm{~F}-\mathrm{NMR}$ spectra were observed in the ${ }^{1} \mathrm{H}$-decoupled mode. NMR yield was determined by ${ }^{19} \mathrm{~F}$ NMR using trifluoromethylbenzene as an internal standard before working up the reaction. High-resolution mass spectra (HRMS) were obtained from Shimadzu LCMS-IT-TOF mass spectrometer and DIONEX UltiMate 3000 \& Bruker Compact TOF mass spectrometer.

\section{Starting Materials}

\subsection{General Procedure for Synthesis of Enol Acetates ${ }^{11}$}

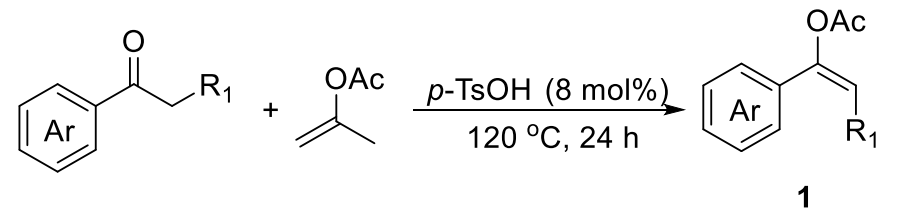

A $100 \mathrm{~mL}$ round-bottom flask equipped with a magnetic stir bar and a reflux condenser was charged with ketone (50 mmol, 1 equiv), isopropenyl acetate ( $250 \mathrm{mmol}, 5$ equiv) and $p$ - $\mathrm{TsOH}$ (4. mmol, 0.08 equiv). The reaction mixture was heated to $120{ }^{\circ} \mathrm{C}$ in aluminum dry block heater. After 24 hours the reaction mixture was allowed to cool to room temperature and the remaining isopropenyl acetate was subsequently evaporated under reduced pressure. The residue was redissolved in ethyl acetate $(100 \mathrm{~mL})$, washed with $\mathrm{H}_{2} \mathrm{O}(3 \times 50 \mathrm{~mL})$ and dried over $\mathrm{Na}_{2} \mathrm{SO}_{4}$. The solvent was evaporated in vacuo to give a dark red oil. The pure product was obtained by distillation under reduced pressure or by purification on $\mathrm{SiO}_{2}$ column chromatography (DCM/PE, 1/1). All enol acetates 1a-1y were synthesized and purified according to the above procedure and in agreement with reference. ${ }^{2-7}$

\subsection{General Procedure for Synthesis of $\mathrm{CF}_{2} \mathrm{H}$ Reagent $2 \mathrm{a}^{8-9}$}

\section{Potassium 2-bromo-2,2-difluoroacetate (S1):}<smiles>[X]OC(=O)C(F)(F)Br</smiles>

A $100 \mathrm{~mL}$ round-bottom flask equipped with a magnetic stir bar was charged with potassium hydroxide 
(4.03 g, $71.7 \mathrm{mmol}, 1$ equiv) was dissolved in $\mathrm{MeOH}(70 \mathrm{~mL})$ at $0{ }^{\circ} \mathrm{C}$. Ethyl bromo 2,2-difluoroacetate $\left(9.48 \mathrm{~mL}, 15.0 \mathrm{~g}, 71.7 \mathrm{mmol}, 1\right.$ equiv) was added slowly to the reaction mixture at $0{ }^{\circ} \mathrm{C}$. The reaction mixture was slowly allowed to warm up to room temperature overnight. The solvent was removed under reduced pressure and compound S1 (13.6 g, $63.8 \mathrm{mmol}, 89 \%$ yield) was obtained as a colorless solid.

\section{2,2-Difluoro-2-(triphenylphosphonio)acetate (S2):}<smiles>O=C([O-])C(F)(C(F)(F)P(c1ccccc1)c1ccccc1)P(=O)(c1ccccc1)c1ccccc1</smiles>

A $100 \mathrm{~mL}$ round-bottom flask equipped with a magnetic stir bar was charged with S1 (13.6 g, 63.8 mmol, 1 equiv) and triphenylphosphine (16.7 g, $63.8 \mathrm{mmol}, 1$ equiv) were dissolved in DMF $(80 \mathrm{~mL})$. The reaction mixture was stirred at room temperature overnight. The formed precipitate was filtered off, washed with cold DMF $(3 \times 20 \mathrm{~mL})$, water $(3 \times 20 \mathrm{~mL})$ and ethyl acetate $(3 \times 20 \mathrm{~mL})$. The precipitate was dried to obtain compound $\mathbf{S 2}(12.7 \mathrm{~g}, 35.6 \mathrm{mmol}, 56 \%$ yield) as a colorless solid.

\section{(Difluoromethyl)triphenylphosphonium bromide (2a):}

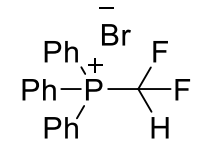

A $100 \mathrm{~mL}$ round-bottom flask equipped with a magnetic stir bar was charged with $\mathbf{S 2}$ (5.04 g, 14.2 mmol, 1 equiv) was dissolved in THF (14 mL). To this mixture $\mathrm{HBr}(48 \%, 1.94 \mathrm{~mL}, 2.87 \mathrm{~g}, 17.0 \mathrm{mmol}$, 1.2 equiv) was added and the reaction mixture was refluxed for 2 hours. The reaction was quenched by addition of water $(50 \mathrm{~mL})$ and DCM $(50 \mathrm{~mL})$. The aqueous layer was extracted with DCM $(2 \times 50 \mathrm{~mL})$. The combined organic layers were dried over $\mathrm{MgSO}_{4}$. Solvent was removed under reduced pressure and the residue was washed multiple times with cold THF $(3 \times 10 \mathrm{~mL})$ to obtain compound $\mathbf{2 a}(4.8 \mathrm{~g}$, $12.2 \mathrm{mmol}, 86 \%$ yield) as a colorless solid.

\subsection{General Procedure for Synthesis of Photocatalyst fac-Ir(ppy)3}

\section{Synthesis of $\left[(\mathrm{ppy})_{2} \mathrm{Ir}-\mu-\mathrm{Cl}\right]_{2}$ :}<smiles></smiles> 
$\left[(\text { ppy })_{2}[\mathrm{r}-\mu-\mathrm{Cl}]_{2}\right.$ was prepared according to the previous literature. ${ }^{10} \mathrm{~A}$ mixture of iridium chloride hydrate $(388 \mathrm{mg}, 1.2 \mathrm{mmol})$ and 2-phenylpyridine $(760 \mathrm{mg}, 4.8 \mathrm{mmol})$ in 2-methoxyethanol/ $\mathrm{H}_{2} \mathrm{O}(30$ $\mathrm{mL} / 10 \mathrm{~mL}$ ) was stirred at $120{ }^{\circ} \mathrm{C}$ under argon for 24 hours in a $10 \mathrm{~mL}$ Schlenk tube equipped with a magnetic stir bar. After cooling to room temperature, the precipitate was filtered off, washed with water and acetone, then dried in vacuo to afford $\left[(\mathrm{ppy})_{2} \mathrm{Ir}-\mu-\mathrm{Cl}\right]_{2}(533 \mathrm{mg}, 83 \%$ yield) as a bright yellow powder.

\section{Synthesis of $f a c-\operatorname{Ir}(\mathrm{ppy})_{3}$ :}

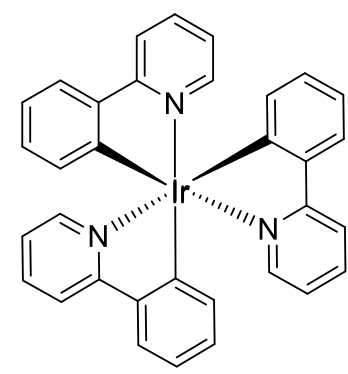

$f a c-\operatorname{Ir}(\text { ppy })_{3}$ was prepared according to the previous literature. ${ }^{10}\left[(\text { ppy })_{2} \mathrm{Ir}-\mu-\mathrm{Cl}\right]_{2}(429 \mathrm{mg}, 0.4 \mathrm{mmol})$, 2-phenylpyridine (186 mg, $1.2 \mathrm{mmol}$ ), and $\mathrm{K}_{2} \mathrm{CO}_{3}(554 \mathrm{mg}, 4 \mathrm{mmol})$ were stirred in glycerol (33 mL) at $200{ }^{\circ} \mathrm{C}$ under argon for 20 hours in a $10 \mathrm{~mL}$ Schlenk tube equipped with a magnetic stir bar. After cooling to room temperature, water was added. The crude product was purified by flash column chromatography on silica gel (DCM/PE, 1/2) to afford $f a c-\operatorname{Ir}(\mathrm{ppy})_{3}(351 \mathrm{mg}, 67 \%$ yield) as a bright yellow powder.

\section{Detailed Optimization of Reaction Conditions}

\subsection{General Procedure}<smiles>[R]C=C(O[C+])c1ccccc1</smiles>

1

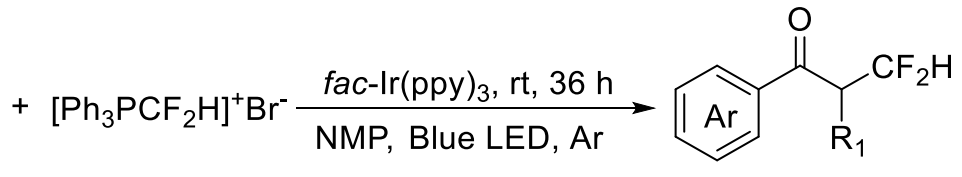

$2 a$

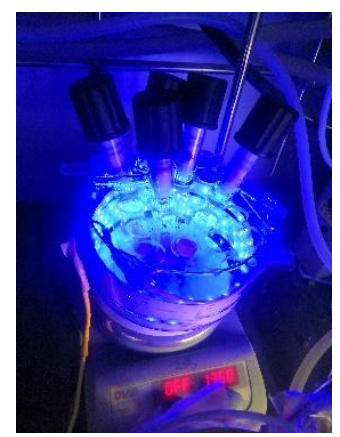

A $10 \mathrm{~mL}$ Schlenk tube equipped with a magnetic stir bar was charged with $0.2 \mathrm{mmol}$ enol acetate 1, 0.4 mmol (difluoromethyl)triphenylphosphonium bromide $2 \mathbf{a}, 3$ mol\% of photocatalysis $f a c-\operatorname{Ir}(\mathrm{ppy})_{3}$ and 2 mL NMP under argon atmosphere. The flask was sealed by a plastic screw-cap with a Teflon sealed inlet for a glass rod. A blue LED was attached to the top of the glass rod, which then could act as an optical fiber. After irradiation at room temperature for 36 hours, the blue LED was removed. The solvent was poured into $20 \mathrm{~mL}$ water and extracted with $20 \mathrm{~mL}$ ethyl ether for three times. The combined organic layer was then washed with $20 \mathrm{~mL}$ brine for three times, dried with $\mathrm{MgSO}_{4}$ and evaporated to give the crude product, which was then purified on silica gel chromatography to afford target compounds $\mathbf{3}$. 
Table S1. Screening of Solvent $\mathrm{t}^{\mathrm{a}}$

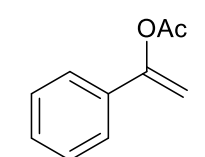

$1 \mathrm{a}$

$+\left[\mathrm{Ph}_{3} \mathrm{PCF}_{2} \mathrm{H}\right]^{+} \mathrm{Br}^{-} \frac{\text { fac-Ir(ppy })_{3}, \mathrm{rt}, 36 \mathrm{~h}}{\text { Solvent, Blue LED, } \mathrm{Ar}}$

2a

$3 \mathbf{a}$

\begin{tabular}{|c|c|c|c|c|}
\hline Entry & PC & $2 a$ & Solvent & Yield $(\%)^{b}$ \\
\hline 1 & $f a c-\operatorname{Ir}(p p y)_{3}$ & 1.5 & $\mathrm{MeCN}$ & trace \\
\hline 2 & $f a c-\operatorname{Ir}(p p y)_{3}$ & 1.5 & $\mathrm{DCM}$ & trace \\
\hline 3 & $f a c-\operatorname{Ir}(p p y)_{3}$ & 1.5 & DCE & trace \\
\hline 4 & $f a c-\operatorname{Ir}(p p y)_{3}$ & 1.5 & Toluene & trace \\
\hline 5 & $f a c-\operatorname{Ir}(p p y)_{3}$ & 1.5 & DMF & 46 \\
\hline 6 & $f a c-\operatorname{Ir}(p p y)_{3}$ & 1.5 & DMAC & 64 \\
\hline 7 & $f a c-\operatorname{Ir}(p p y)_{3}$ & 1.5 & DMSO & 86 \\
\hline 8 & $f a c-I r(p p y)_{3}$ & 1.5 & NMP & 88 \\
\hline 9 & $f a c-\operatorname{Ir}(p p y)_{3}$ & 1.5 & THF & 74 \\
\hline 10 & fac-Ir(ppy) 3 & 1.5 & Acetone & mess \\
\hline
\end{tabular}

[a] Reaction conditions: 1 a $(0.2 \mathrm{mmol}, 1$ equiv), 2 a $(0.3 \mathrm{mmol}, 1.5$ equiv), PC (0.006 $\mathrm{mmol}, 3 \mathrm{~mol} \%)$, solvent $(2 \mathrm{~mL})$, room temperature, $36 \mathrm{~h}$, argon atmosphere, $15 \mathrm{~W}$ blue LED.

[b] Isolated yield.

Table S2. Screening of Ratio of $1 \mathrm{a}$ and $2 \mathrm{a}^{\mathrm{a}}$<smiles>C=C(OC(C)=O)c1ccccc1</smiles>

$1 \mathrm{a}$

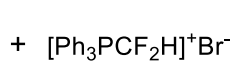

$2 a$

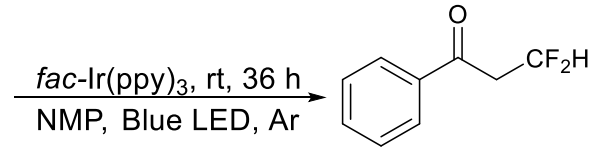

$3 a$

\begin{tabular}{lccll}
\hline Entry & PC & 2a & Solvent & Yield $(\%)^{\mathrm{b}}$ \\
\hline 1 & $\mathrm{fac}-\operatorname{Ir}(\mathrm{ppy})_{3}$ & 1.5 & NMP & 88 \\
2 & $\mathrm{fac}-\operatorname{Ir}(\mathrm{ppy})_{3}$ & $\mathbf{2}$ & NMP & $\mathbf{8 9}$ \\
3 & $\mathrm{fac}-\operatorname{Ir}(\mathrm{ppy})_{3}$ & 3 & NMP & 85 \\
\hline
\end{tabular}

[a] Reaction conditions: $1 \mathrm{a}(0.2 \mathrm{mmol}, 1$ equiv), PC ( $0.006 \mathrm{mmol}, 3 \mathrm{~mol} \%)$, NMP (2 $\mathrm{mL}$ ), room temperature, $36 \mathrm{~h}$, argon atmosphere, $15 \mathrm{~W}$ blue LED.

[b] Isolated yield. 
Table S3. Screening of $\mathbf{P C}^{\text {a }}$

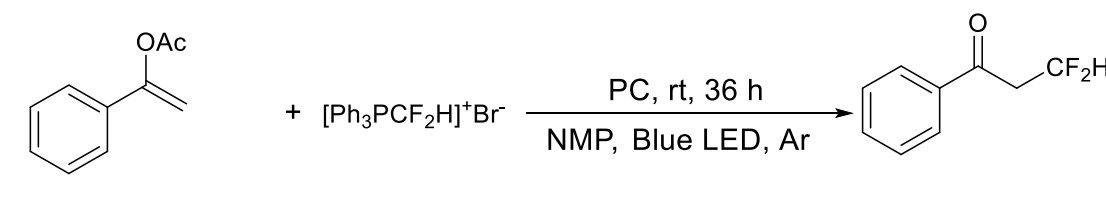

$1 \mathbf{a}$

$2 a$

$3 a$

\begin{tabular}{lcccc}
\hline Entry & PC & $\mathbf{2 a}$ & Solvent & Yield $(\%)^{\mathbf{b}}$ \\
\hline 1 & fac-Ir(ppy) $)_{3}$ & $\mathbf{2}$ & NMP & $\mathbf{8 9}$ \\
2 & Eosin Y Disodium Salt & 2 & NMP & trace \\
3 & $4 \mathrm{CzIPN}$ & 2 & NMP & trace \\
4 & $\mathrm{Ru}(\mathrm{bpy})_{2} \mathrm{Cl}_{2}$ & 2 & NMP & 0 \\
5 & Solvent Red 43 & 2 & NMP & 0 \\
\hline
\end{tabular}

[a] Reaction conditions: 1 a $(0.2 \mathrm{mmol}, 1$ equiv), 2a (0.4 mmol, 2 equiv), PC (0.006 mmol, $3 \mathrm{~mol} \%)$, NMP $(2 \mathrm{~mL})$, room temperature, $36 \mathrm{~h}$, argon atmosphere, $15 \mathrm{~W}$ blue LED.

[b] Isolated yield.

Table S4. Screening of Base ${ }^{\mathrm{a}}$<smiles>C=C(OC(C)=O)c1ccccc1</smiles>

1a
$+\left[\mathrm{Ph}_{3} \mathrm{PCF}_{2} \mathrm{H}\right]^{+} \mathrm{Br}^{-} \stackrel{\text { fac-Ir(ppy })_{3}, \mathrm{rt}, 36 \mathrm{~h}, \text { Base }}{\mathrm{NMP}, \text { Blue LED, Ar }}$

$2 a$

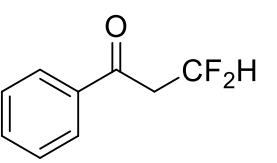

$3 a$

\begin{tabular}{cccccc}
\hline Entry & PC & $\mathbf{2 a}$ & Base & Solvent & ${\text { Yield }(\%)^{\mathrm{b}}}^{\mathrm{b}}$ \\
\hline $\mathbf{f}$ & $\mathrm{fac}-\operatorname{Ir}(\mathrm{ppy})_{3}$ & $\mathbf{2}$ & $-\cdots$ & $\mathrm{NMP}$ & $\mathbf{8 9}$ \\
2 & $\mathrm{fac}-\operatorname{Ir}(\mathrm{ppy})_{3}$ & 2 & $\mathrm{NEt}_{3}$ & $\mathrm{NMP}$ & 79 \\
3 & $\mathrm{fac}-\operatorname{Ir}(\mathrm{ppy})_{3}$ & 2 & $\mathrm{~K}_{2} \mathrm{CO}_{3}$ & $\mathrm{NMP}$ & 51 \\
4 & $\mathrm{fac}-\operatorname{Ir}(\mathrm{ppy})_{3}$ & 2 & $\mathrm{DBU}$ & $\mathrm{NMP}$ & 66 \\
5 & $\mathrm{fac}-\operatorname{Ir}(\mathrm{ppy})_{3}$ & 2 & $\mathrm{Py}$ & $\mathrm{NMP}$ & 76 \\
6 & $\mathrm{fac}-\operatorname{Ir}(\mathrm{ppy})_{3}$ & 2 & $\mathrm{~K}_{2} \mathrm{PO}_{4}$ & $\mathrm{NMP}$ & 74
\end{tabular}

[a] Reaction conditions: $1 \mathrm{a}\left(0.2 \mathrm{mmol}, 1\right.$ equiv), $2 \mathrm{a}(0.4 \mathrm{mmol}, 2 \text { equiv), fac-Ir(ppy) })_{3}(0.006 \mathrm{mmol}, 3 \mathrm{~mol} \%)$, NMP ( $2 \mathrm{~mL}$ ), base (2 equiv), room temperature, $36 \mathrm{~h}$, argon atmosphere, $15 \mathrm{~W}$ blue LED.

[b] Isolated yield. 
Table S5. In the Dark and without $\mathrm{PC}^{\mathrm{a}}$<smiles>C=C(OC)c1ccccc1</smiles>

1a
$+\left[\mathrm{Ph}_{3} \mathrm{PCF}_{2} \mathrm{H}\right]^{+} \mathrm{Br} \frac{\mathrm{PC}, \mathrm{rt}, 36 \mathrm{~h}}{\mathrm{NMP}, \mathrm{Blue} \mathrm{LED,} \mathrm{Ar}}$

2a
$3 \mathbf{a}$

\begin{tabular}{lcccc}
\hline Entry & $\mathrm{PC}$ & $\mathbf{2 a}$ & Solvent & ${\text { Yield }(\%)^{\mathrm{b}}}^{\mathrm{a}}$ \\
\hline 1 & $\mathrm{fac}-\operatorname{Ir}(\mathrm{ppy})_{3}$ & $\mathbf{2}$ & NMP & $\mathbf{8 9}$ \\
2 & --- & 2 & NMP & 0 \\
$3^{\mathrm{c}}$ & fac-Ir(ppy $)_{3}$ & 2 & NMP & 0 \\
\hline
\end{tabular}

[a] Reaction conditions: $1 \mathrm{a}(0.2 \mathrm{mmol}, 1$ equiv), 2a ( $0.4 \mathrm{mmol}, 2$ equiv), PC (0.006 mmol, $3 \mathrm{~mol} \%)$, NMP ( $2 \mathrm{~mL})$, room temperature, $36 \mathrm{~h}$, argon atmosphere, $15 \mathrm{~W}$ blue LED.

[b] Isolated yield.

[c] In the dark.

\section{Investigation of Reaction Mechanism}

\subsection{BHT Trapping Experiment}<smiles>C=C(OC(C)=O)c1ccccc1</smiles>

$1 a$
$+\left[\mathrm{Ph}_{3} \mathrm{PCF}_{2} \mathrm{H}^{+} \mathrm{Br}^{-}\right.$

$2 a$<smiles>O=C(CC(F)(F)F)c1ccccc1</smiles>

$3 a, 45 \%$ isolated yield

A $10 \mathrm{~mL}$ Schlenk tube equipped with a magnetic stir bar was charged with 1a (32.4 mg, $0.2 \mathrm{mmol}), \mathbf{2 a}$ (157.3 mg, $0.4 \mathrm{mmol}$ ), BHT (2 equiv), $3 \mathrm{~mol} \%$ photocatalysis $f a c-\operatorname{Ir}(\mathrm{ppy})_{3}$ and $2 \mathrm{~mL} \mathrm{NMP} \mathrm{under} \mathrm{argon}$ atmosphere. The flask was sealed by a plastic screw-cap with a Teflon sealed inlet for a glass rod. A blue LED was attached to the top of the glass rod, which then could act as an optical fiber. After irradiation at room temperature for 36 hours, the blue LED was removed. The solvent was poured to 20 $\mathrm{mL}$ water and extracted with $20 \mathrm{~mL}$ ethyl ether for three times. The combined organic layer was then washed with $20 \mathrm{~mL}$ water for three times, dried with $\mathrm{MgSO}_{4}$ and evaporated giving the crude product, which was purified on silica gel chromatography $(\mathrm{DCM} / \mathrm{PE}=1 / 2)$ to give target compounds $3 \mathbf{a}$ as colorless oil in $45 \%$ yield. 


\subsection{TEMPO Trapping Experiment ${ }^{11}$}

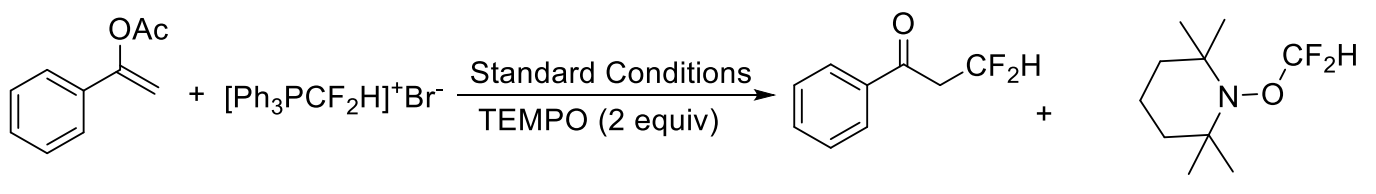

1a

2a

3a, $12 \%{ }^{19} \mathrm{~F}$ NMR yield Detected by ${ }^{19} \mathrm{~F}$ NMR

A $10 \mathrm{~mL}$ Schlenk tube equipped with a magnetic stir bar was charged with $\mathbf{1 a}(32.4 \mathrm{mg}, 0.2 \mathrm{mmol}), \mathbf{2 a}$ ( $157.3 \mathrm{mg}, 0.4 \mathrm{mmol}$ ), TEMPO ( 2 equiv), $3 \mathrm{~mol} \%$ photocatalysis $f a c$-Ir(ppy) 3 and $2 \mathrm{~mL}$ NMP under argon atmosphere. The flask was sealed by a plastic screw-cap with a Teflon sealed inlet for a glass rod. A blue LED was attached to the top of the glass rod, which then could act as an optical fiber. After irradiation at room temperature for 36 hours, the blue LED was removed. The trifluorotoluene was dripped into the mixture. The desired product 3a in $12 \%{ }^{19} \mathrm{~F}$ NMR yield and the previous literature were also detected by ${ }^{19} \mathrm{~F}$ NMR spectroscopy.
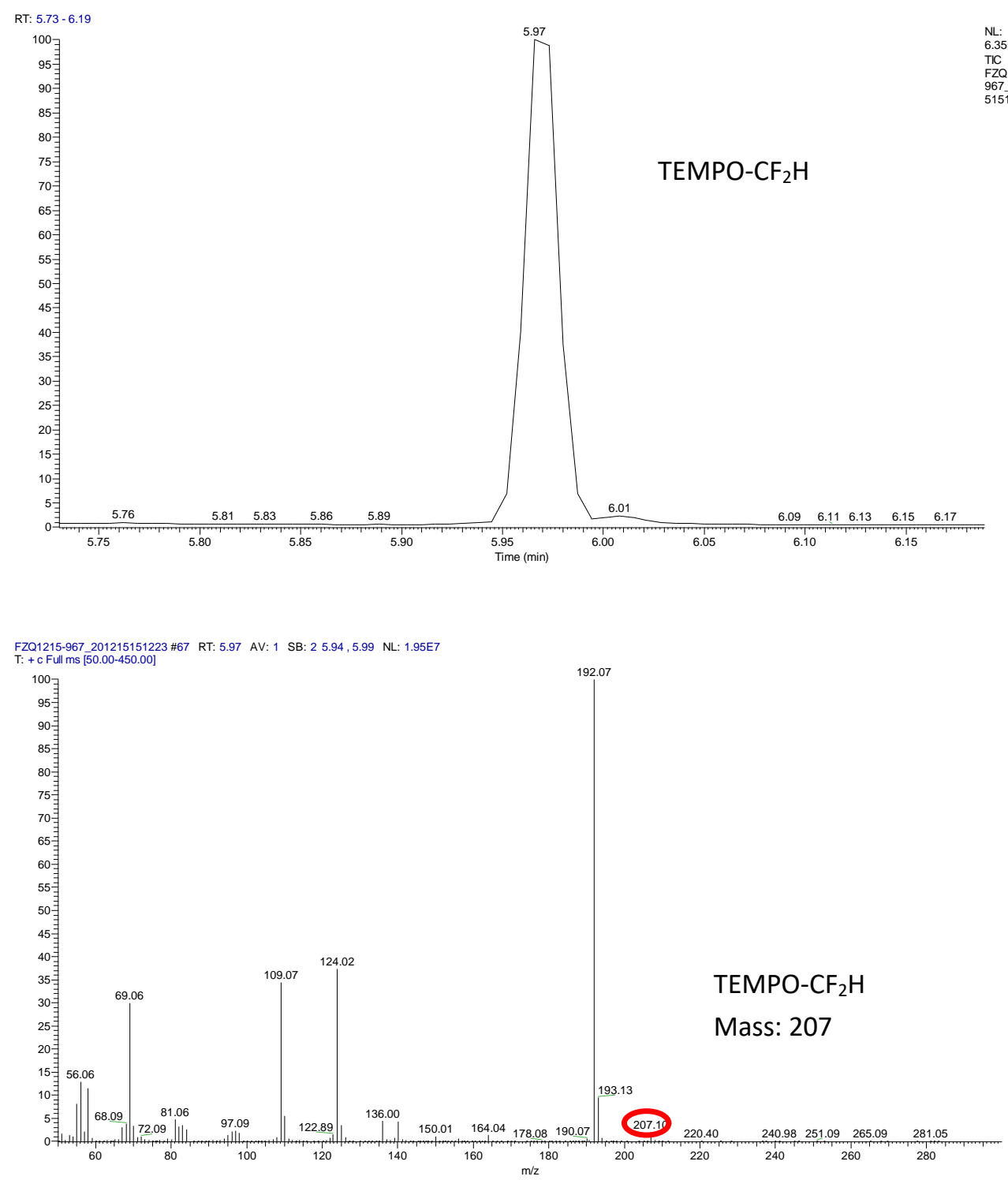


\section{Derivatization}

\subsection{Synthesis of 3,3-difluoro-1-(p-tolyl)propan-1-ol (4) ${ }^{12}$}

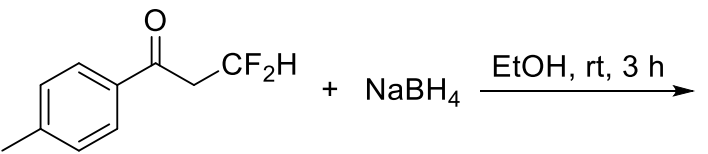

$3 \mathbf{b}$<smiles>Cc1ccc(C(O)CC(F)F)cc1</smiles>

$4,95 \%$ yield

A $10 \mathrm{~mL}$ Schlenk tube equipped with a magnetic stir bar was charged with $\mathbf{3 b}(82.0 \mathrm{mg}, 0.45 \mathrm{mmol})$, $\mathrm{NaBH}_{4}(18.6 \mathrm{mg}, 0.51 \mathrm{mmol})$ and ethanol $(5 \mathrm{~mL})$ under argon. The reaction mixture was stirred at room temperature for 3 hours. The product mixture was concentrated in vacuo and purified by column chromatography $(\mathrm{EA} / \mathrm{PE}=1 / 5)$ to afford 4 as a colorless oil $(79.6 \mathrm{mg}, 95 \%$ yield $)$.

\subsection{Synthesis of 4,4-difluoro-2-(p-tolyl)-2-((trimethylsilyl)oxy)butanenitrile (5) ${ }^{13}$}

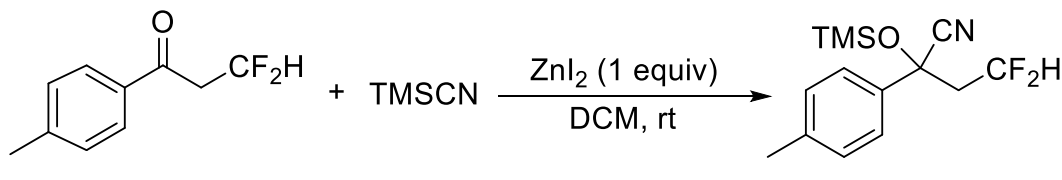

A $10 \mathrm{~mL}$ Schlenk tube equipped with a magnetic stir bar was charged with $\mathbf{3 b}(36.8 \mathrm{mg}, 0.2 \mathrm{mmol})$, $\mathrm{ZnI}_{2}(64.0 \mathrm{mg}, 0.2 \mathrm{mmol})$ and DCM $(2 \mathrm{~mL})$ under argon. With vigorous stirring, trimethylsilylcyanide (49.3 $\mathrm{mg}, 0.51 \mathrm{mmol}$ ) was added dropwise. The reaction mixture was stirred under argon at room temperature for 24 hours. The mixture was diluted with chloroform $(10 \mathrm{~mL})$, washed with sat. $\mathrm{NaHCO}_{3}$ aqueous, dried over $\mathrm{MgSO}_{4}$ and filtered. The filtrate was concentrated in vacuo and purified by column chromatography $(\mathrm{PE} / \mathrm{DCM}=3 / 1)$ to afford 5 as a colorless oil (50.9 $\mathrm{mg}, 90 \%$ yield).

\subsection{Synthesis of 4,4-difluoro-2-(p-tolyl)-2-((trimethylsilyl)oxy)butanamide (6) ${ }^{14}$}

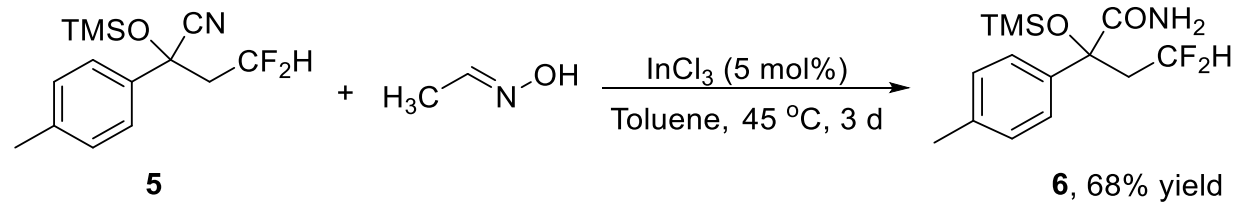

A stirred solution of 5 (56.6 mg, $0.2 \mathrm{mmol})$, acetaldoxime $(33.6 \mathrm{mg}, 0.6 \mathrm{mmol})$, and $\mathrm{InCl}_{3}(1.86 \mathrm{mg}$, $0.01 \mathrm{mmol})$ in toluene $(1 \mathrm{ml})$ was heated at $45^{\circ} \mathrm{C}$ in aluminum dry block heater for 3 days in a $10 \mathrm{~mL}$ Schlenk tube equipped with a magnetic stir bar. After removal of the solvent, the residue was purified by column chromatography $(\mathrm{PE} / \mathrm{EA}=2 / 1)$ to afford 6 as a colorless oil $(41.0 \mathrm{mg}, 68 \%$ yield). 


\subsection{Reaction of the $\alpha-\mathrm{CF}_{2} \mathrm{H}$ ketone with Aniline}

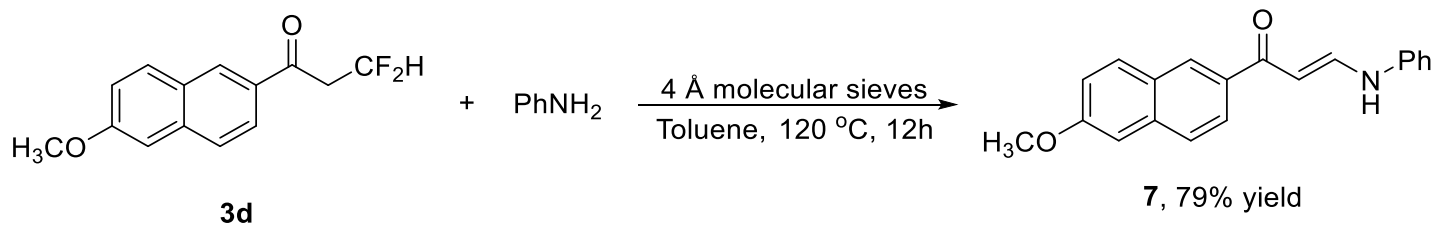

Under inert atmosphere, a $10 \mathrm{~mL}$ Schlenk tube equipped with a magnetic stir bar was charged with 3d (50.0 mg, $0.2 \mathrm{mmol})$, aniline $(37.3 \mathrm{mg}, 0.4 \mathrm{mmol})$, and $4 \AA$ molecular sieves $(200 \mathrm{mg})$ in toluene (1 $\mathrm{mL}$ ). The reaction mixture was heated to $120{ }^{\circ} \mathrm{C}$ in aluminum dry block heater for 12 hours. After removal of the solvent, the residue was purified by column chromatography $(\mathrm{DCM} / \mathrm{PE}=1 / 1)$ to afford 7 as a pale yellow solid (47.9 $\mathrm{mg}, 79 \%$ yield).

\subsection{Gram-scale Reaction}

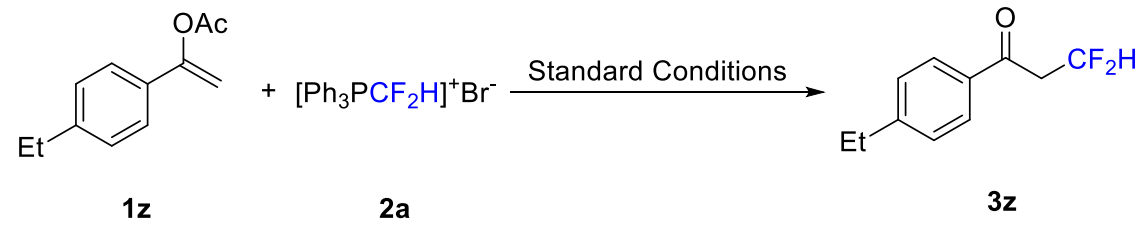

A $250 \mathrm{~mL}$ round-bottom flask equipped with a magnetic stir bar as charged with $\mathbf{1 z}(1.53 \mathrm{~g}, 8 \mathrm{mmol})$, 2a $(6.28 \mathrm{~g}, 1.6 \mathrm{mmol})$, photocatalysis $f a c-\operatorname{Ir}(\mathrm{ppy})_{3}(156.8 \mathrm{mg}, 0.24 \mathrm{mmol})$ and $80 \mathrm{~mL}$ NMP under argon atmosphere. A blue LED was attached to the top of the glass rod, which then could act as an optical fiber. After irradiation at room temperature for 72 hours, the blue LED was removed. The solvent was poured into $50 \mathrm{~mL}$ water and extracted with $50 \mathrm{~mL}$ ethyl ether for three times. The combined organic layer was then washed with $50 \mathrm{~mL}$ brine for three times, dried with $\mathrm{MgSO}_{4}$ and evaporated to give the crude product, which was then purified on silica gel chromatography (DCM/PE $=1 / 2)$ to afford target compounds $3 z$ (1.05 g, 66\%).

\subsection{1-Phenylvinyl Trifluoromethanesulfonate as a Substrate}

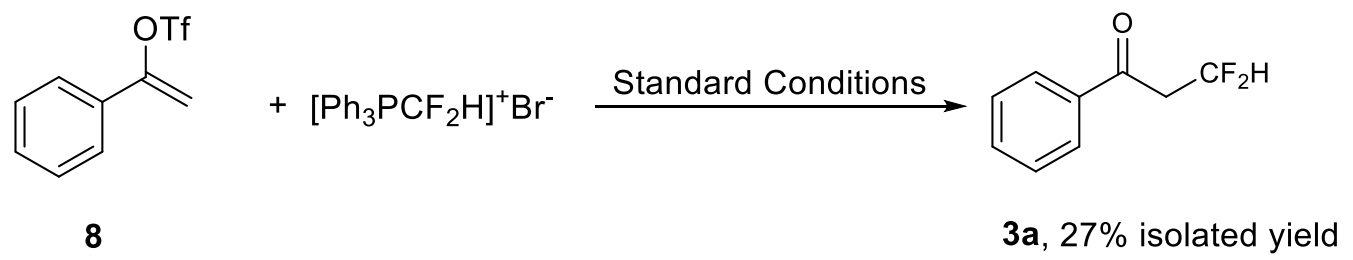

A $10 \mathrm{~mL}$ Schlenk tube equipped with a magnetic stir bar was charged with $(50.4 \mathrm{mg}, 0.2 \mathrm{mmol})$ 1-phenylethenyl trifluoromethanesulfonate $\mathbf{8}$ (commercially available), $0.4 \mathrm{mmol}$ (difluoromethyl)triphenylphosphonium bromide $\mathbf{2 a}, 3 \mathrm{~mol} \%$ of photocatalysis $f a c-\operatorname{Ir}(\mathrm{ppy})_{3}$ and $2 \mathrm{~mL}$ NMP under argon atmosphere. The flask was sealed by a plastic screw-cap with a Teflon sealed inlet for a glass rod. A blue LED was attached to the top of the glass rod, which then could act as an optical 
fiber. After irradiation at room temperature for 36 hours, the blue LED was removed. The solvent was poured into $20 \mathrm{~mL}$ water and extracted with $20 \mathrm{~mL}$ ethyl ether for three times. The combined organic layer was then washed with $20 \mathrm{~mL}$ brine for three times, dried with $\mathrm{MgSO}_{4}$ and evaporated to give the crude product, which was then purified on silica gel chromatography (DCM/PE $=1 / 2)$ to afford target compounds 3a (9.2 $\mathrm{mg}, 27 \%$ isolated yield).

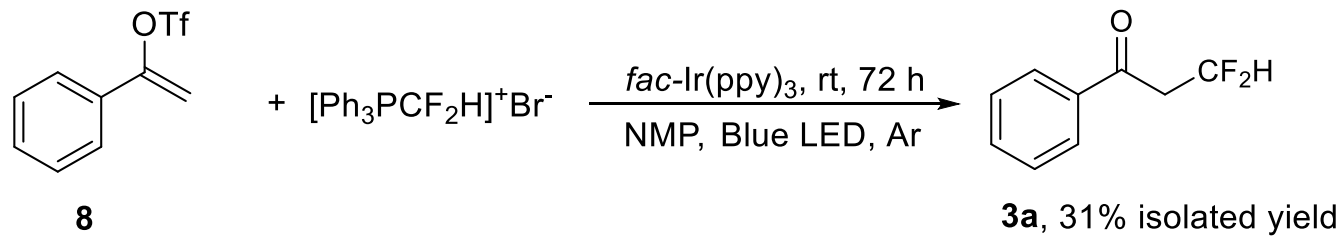

A $10 \mathrm{~mL}$ Schlenk tube equipped with a magnetic stir bar was charged with $(50.4 \mathrm{mg}, 0.2 \mathrm{mmol})$ $\begin{array}{llllll}\text { trifluoromethanesulfonate } & 8 & \text { (commercially } & \text { available), } & 0.4 & \mathrm{mmol}\end{array}$ (difluoromethyl)triphenylphosphonium bromide $2 \mathbf{a}, 3 \mathrm{~mol} \%$ of photocatalysis $f a c-\operatorname{Ir}(\mathrm{ppy})_{3}$ and $2 \mathrm{~mL}$ NMP under argon atmosphere. The flask was sealed by a plastic screw-cap with a Teflon sealed inlet for a glass rod. A blue LED was attached to the top of the glass rod, which then could act as an optical fiber. After irradiation at room temperature for 72 hours, the blue LED was removed. The solvent was poured into $20 \mathrm{~mL}$ water and extracted with $20 \mathrm{~mL}$ ethyl ether for three times. The combined organic layer was then washed with $20 \mathrm{~mL}$ brine for three times, dried with $\mathrm{MgSO}_{4}$ and evaporated to give the crude product, which was then purified on silica gel chromatography (DCM/PE $=1 / 2)$ to afford target compounds 3a (10.6 mg, 31\% isolated yield).

\subsection{Trimethyl((1-phenylvinyl)oxy)silane as a Substrate}

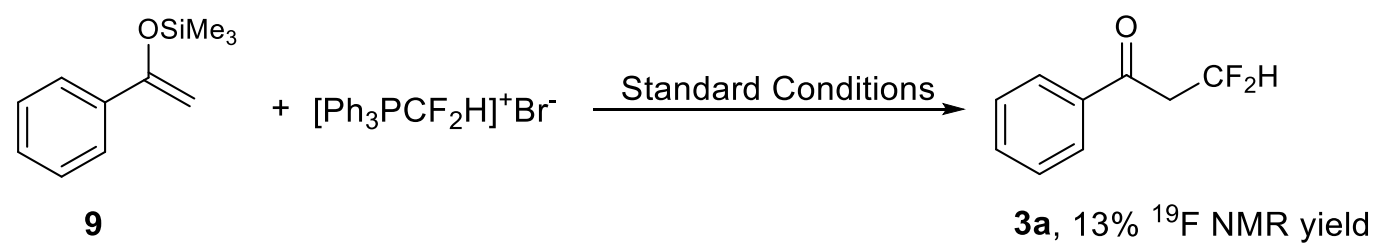

A $10 \mathrm{~mL}$ Schlenk tube equipped with a magnetic stir bar was charged with 1-Phenyl-1-triMethylsilyloxyethylene (38.5 mg, $0.2 \mathrm{mmol}$ ), $2 \mathrm{a}(157.3 \mathrm{mg}, 0.4 \mathrm{mmol}), 3 \mathrm{~mol} \%$ photocatalysis $f a c-\operatorname{Ir}(\text { ppy })_{3}$ and $2 \mathrm{~mL}$ NMP under argon atmosphere. The flask was sealed by a plastic screw-cap with a Teflon sealed inlet for a glass rod. A blue LED was attached to the top of the glass rod, which then could act as an optical fiber. After irradiation at room temperature for 36 hours, the blue LED was removed. The trifluoromethylbenzene was dripped into the mixture. The desired product 3a in $13 \%{ }^{19} \mathrm{~F}$ NMR yield.

\section{Mechanistic Experiments}

We examined the addition of alcohol. Ethanol and 4-phenyl-1-butanol were added in reaction a and $b$ shown below. We obtained target product $3 \mathbf{a}$ in $75 \%$ and $83 \%$ yield, respectively. In reaction $\mathrm{b}$, we found the signal of 4-phenylbutyl acetate via GC-MS. 
a)

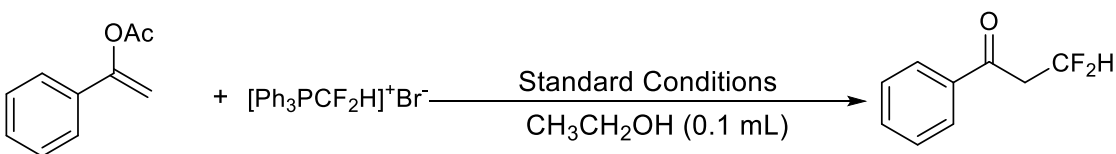

$3 a, 75 \%$ isolated yield

b)

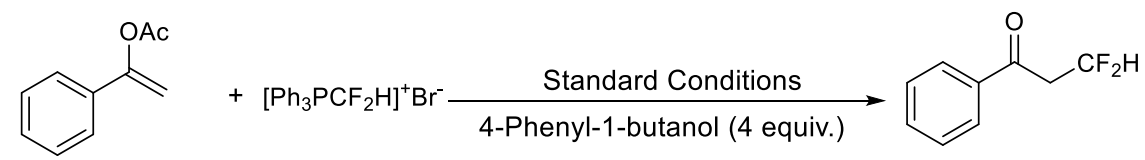

$3 a, 83 \%$ isolated yield

Reaction a: A $10 \mathrm{~mL}$ Schlenk tube equipped with a magnetic stir bar was charged with $\mathbf{1 a}$ (32.4 mg, 0.2 mmol), 2a (157.3 mg, $0.4 \mathrm{mmol}), \mathrm{CH}_{3} \mathrm{CH}_{2} \mathrm{OH}(0.1 \mathrm{~mL}), 3 \mathrm{~mol} \%$ photocatalysis $f a c$ - $\operatorname{Ir}(\mathrm{ppy})_{3}$ and $2 \mathrm{~mL}$ NMP under argon atmosphere. The flask was sealed by a plastic screw-cap with a Teflon sealed inlet for a glass rod. A blue LED was attached to the top of the glass rod, which then could act as an optical fiber. After irradiation at room temperature for 36 hours, the blue LED was removed. The solvent was poured into $50 \mathrm{~mL}$ water and extracted with $50 \mathrm{~mL}$ ethyl ether for three times. The combined organic layer was then washed with $50 \mathrm{~mL}$ brine for three times, dried with $\mathrm{MgSO}_{4}$ and evaporated to give the crude product, which was then purified on silica gel chromatography (DCM/PE $=1 / 2$ ) to afford target compounds $\mathbf{3 y}$ in $75 \%$ isolated yield.

Reaction b: A $10 \mathrm{~mL}$ Schlenk tube equipped with a magnetic stir bar was charged with $1 \mathrm{a}$ (32.4 mg, 0.2 mmol), 2a (157.3 mg, $0.4 \mathrm{mmol})$, 4-Phenyl-1-butanol (4 equiv.), $3 \mathrm{~mol} \%$ photocatalysis $f a c-\operatorname{Ir}(\mathrm{ppy})_{3}$ and $2 \mathrm{~mL}$ NMP under argon atmosphere. The flask was sealed by a plastic screw-cap with a Teflon sealed inlet for a glass rod. A blue LED was attached to the top of the glass rod, which then could act as an optical fiber. After irradiation at room temperature for 36 hours, the blue LED was removed. The solvent was poured into $50 \mathrm{~mL}$ water and extracted with $50 \mathrm{~mL}$ ethyl ether for three times. The combined organic layer was then washed with $50 \mathrm{~mL}$ brine for three times, dried with $\mathrm{MgSO}_{4}$ and evaporated to give the crude product, which was then purified on silica gel chromatography (DCM/PE $=1 / 2$ ) to afford target compounds $\mathbf{3 y}$ in $83 \%$ isolated yield.

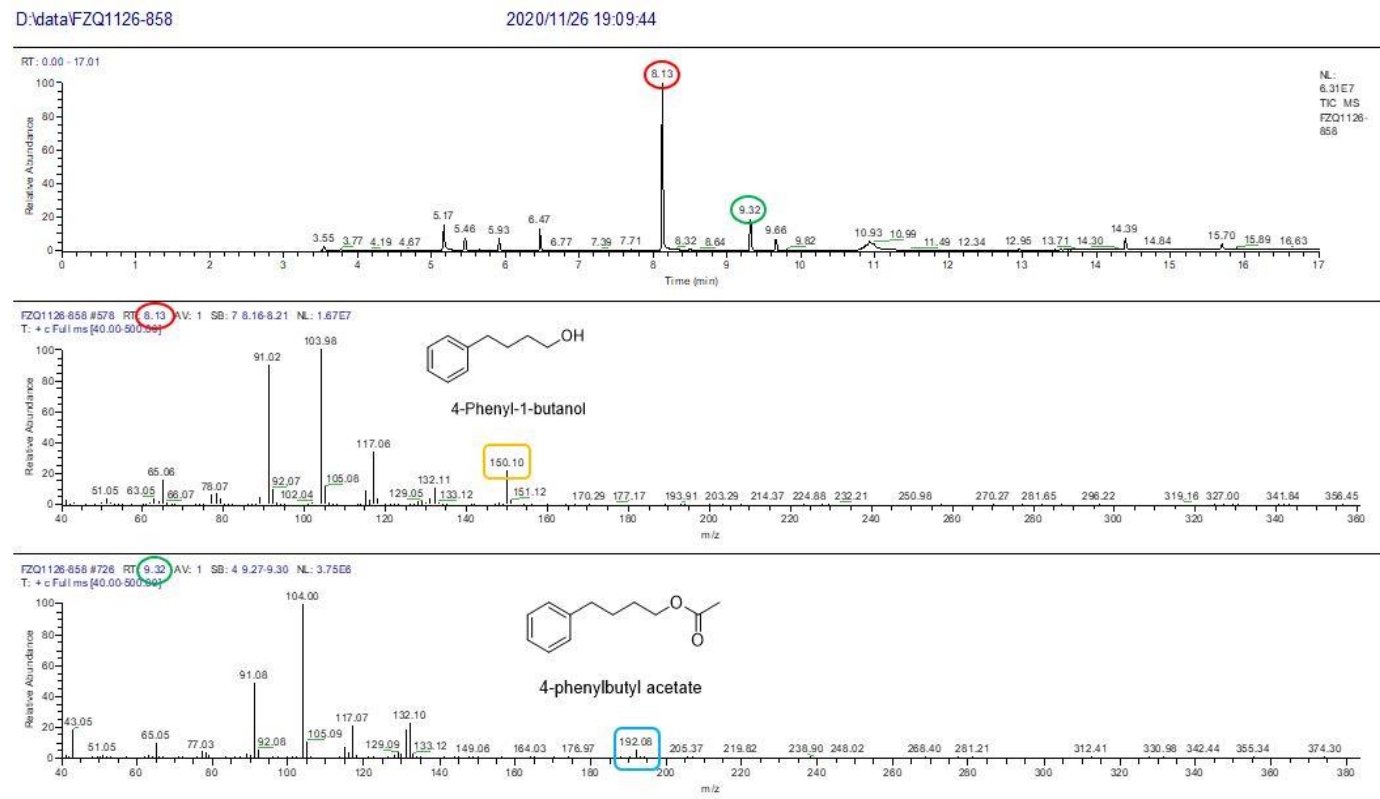




\section{Stern-Volmer Quenching Experiments}

Stern-Volmer fluorescence quenching experiments were run with freshly prepared solution of $5 \times 10^{-5}$ $\mathrm{M}$ solution of $f a c$-Ir(ppy) 3 in dry NMP added the appropriate amount of a quencher in a screw-top quartz cuvette at room temperature. The solutions were irradiated at $370 \mathrm{~nm}$ and fluorescence was measured from $390 \mathrm{~nm}$ to $720 \mathrm{~nm}$. After degassing the sample with a stream of $\mathrm{N}_{2}$ for 10 minutes, the emission of the sample was collected.
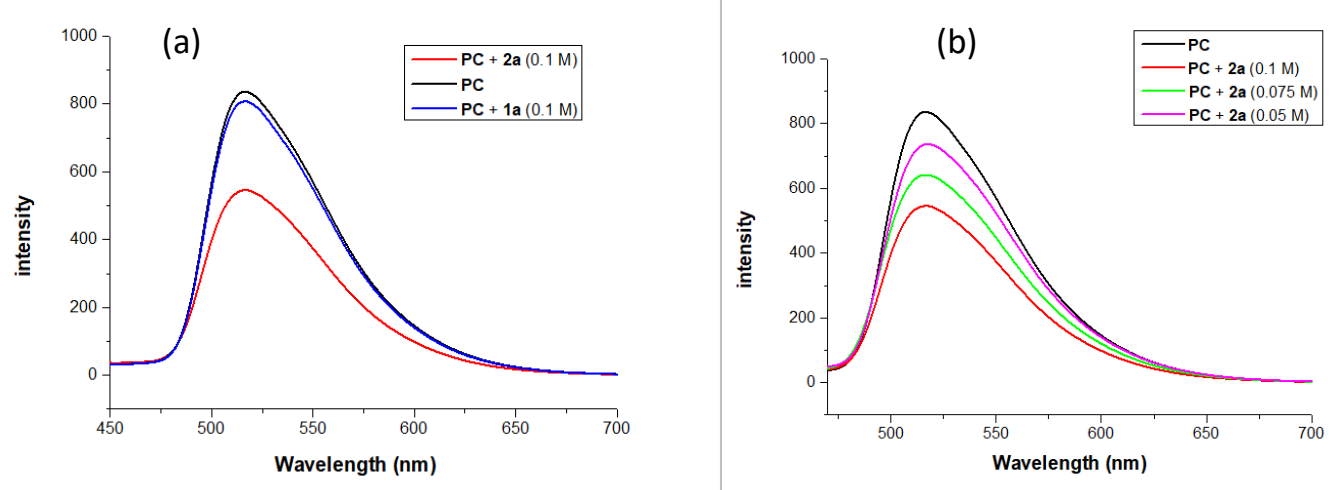

Figure S1. Luminescence quenching study: (a) the emission spectra of a $5 \times 10^{-5} \mathrm{M}$ solution of $f a c-\operatorname{Ir}(\text { ppy })_{3}$ with reactants in degassed anhydrous NMP excited at $370 \mathrm{~nm}$; (b) the emission spectra of a $5 \times 10^{-5} \mathrm{M}$ solution of $f a c-\operatorname{Ir}(\mathrm{ppy})_{3}$ with various concentrations of $\mathbf{2 a}$ in degassed anhydrous NMP excited at $370 \mathrm{~nm}$.

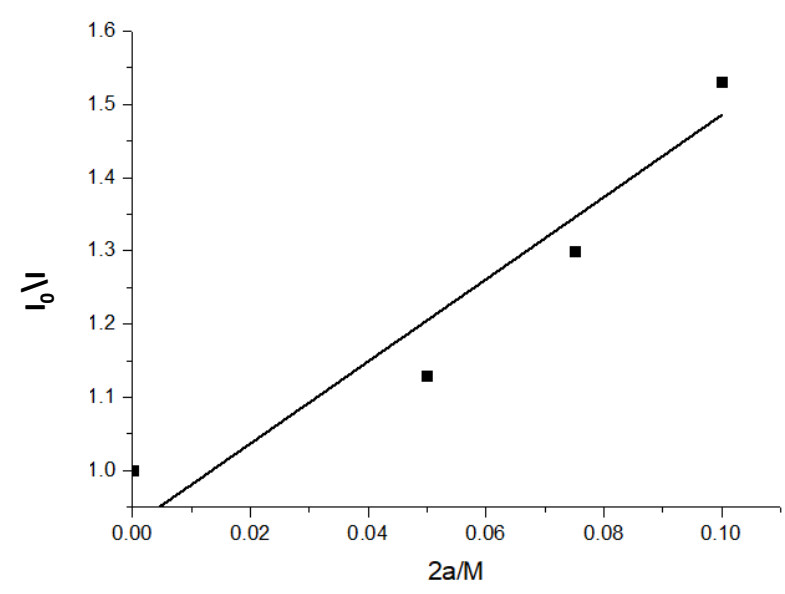

Figure S2. the linear relationship between $\mathrm{I}_{0} / \mathrm{I}$ and the increasing concentration of $\mathbf{2 a}$

Figures S1 and S2 showed an obvious linear relationship between the fluorescence intensities and the concentrations of difluoromethyltriphenylphosphonium bromide 2a. All of these results suggested that the excited state of the photocatalyst was quenched by the substrate difluoromethyltriphenylphosphonium bromide $\mathbf{2 a}$. 


\section{X-ray Crystallographic Data of $\mathbf{3 b}$}
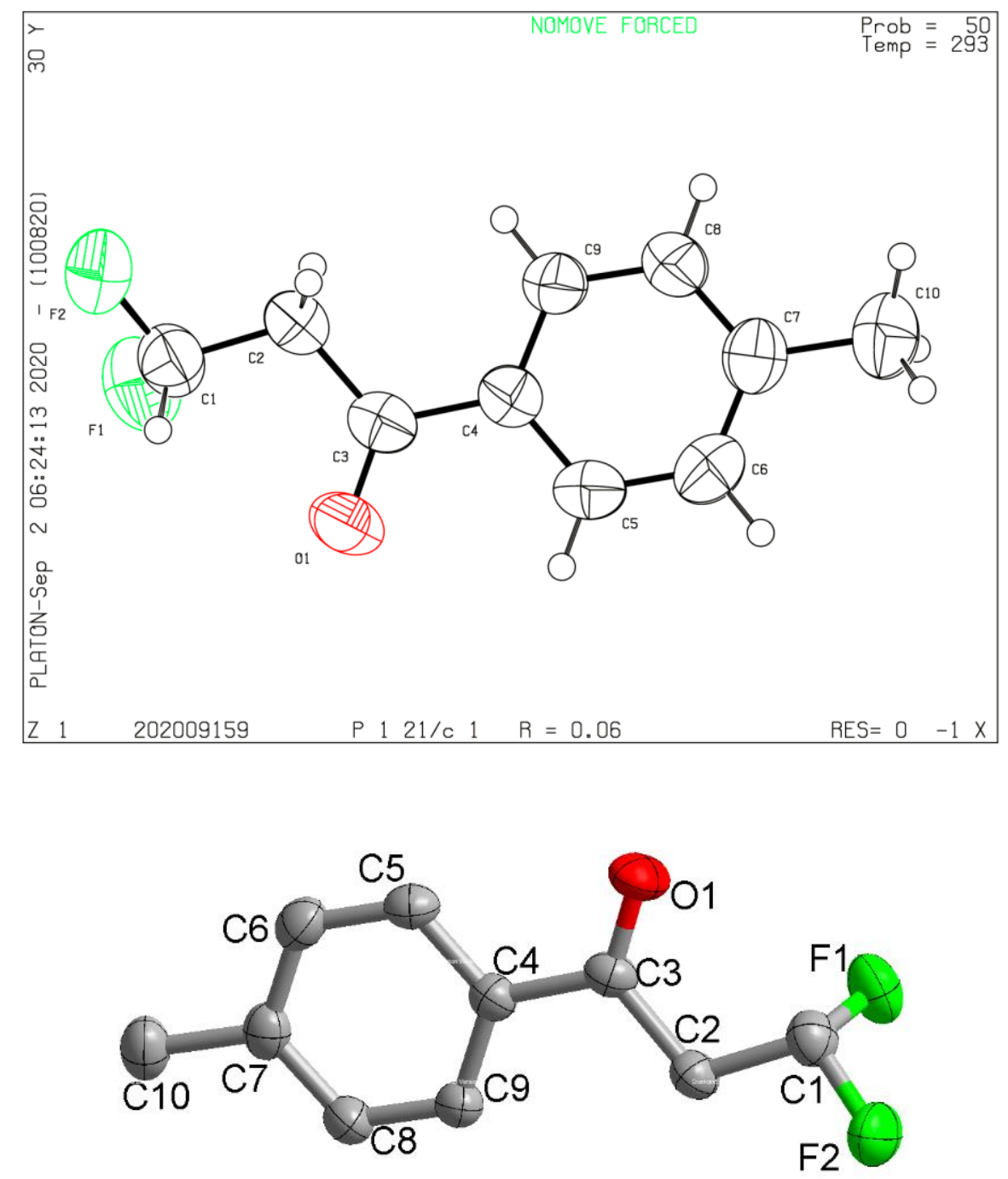

The product $\mathbf{3 b}$ was crystallized from a hexane/DCM mixture $(1 / 3, \mathrm{v} / \mathrm{v})$. The atoms are depicted with $50 \%$ probability ellipsoids. The crystallographic data are summarized in the following table.

\begin{tabular}{|l|l|}
\hline Identification code & $\mathbf{3 b}$ \\
\hline Empirical formula & $\mathrm{C}_{10} \mathrm{H}_{10} \mathrm{~F}_{2} \mathrm{O}$ \\
\hline Formula weight & 184.18 \\
\hline Temperature/K & $293(2)$ \\
\hline Crystal system & monoclinic \\
\hline Space group & $\mathrm{P}{ }_{1} / \mathrm{c}$ \\
\hline $\mathrm{a} / \AA$ & $9.6731(11)$ \\
\hline $\mathrm{b} / \AA$ & $9.4736(9)$ \\
\hline $\mathrm{c} / \AA$ & $10.2537(13)$ \\
\hline$\alpha /{ }^{\circ}$ & 90 \\
\hline
\end{tabular}




\begin{tabular}{|l|l|}
\hline$\beta /{ }^{\circ}$ & $103.811(13)$ \\
\hline$\gamma /{ }^{\circ}$ & 90 \\
\hline Volume $/ \AA^{3}$ & $912.48(18)$ \\
\hline $\mathrm{Z}$ & 4 \\
\hline$\rho_{\text {calc }} / \mathrm{cm}^{3}$ & 1.341 \\
\hline$\mu / \mathrm{mm}^{-1}$ & 0.967 \\
\hline $\mathrm{F}(000)$ & 384.0 \\
\hline Crystal size $/ \mathrm{mm}^{3}$ & $0.15 \times 0.13 \times 0.1$ \\
\hline Radiation & $\mathrm{CuK \alpha}(\lambda=1.54184)$ \\
\hline $2 \Theta$ range for data collection/ & \\
\hline Index ranges & 9.416 to 134.144 \\
\hline Reflections collected & $-8 \leqslant \mathrm{~h} \leqslant 11,-9 \leqslant \mathrm{k} \leqslant 11,-10 \leqslant 1 \leqslant 12$ \\
\hline Independent reflections & 3330 \\
\hline Data/restraints/parameters & $1632\left[\mathrm{R}_{\text {int }}=0.0269, \mathrm{R}_{\text {sigma }}=0.0409\right]$ \\
\hline Goodness-of-fit on $\mathrm{F}^{2}$ & $1632 / 0 / 119$ \\
\hline Final $\mathrm{R}$ indexes [I $>=2 \sigma(\mathrm{I})]$ & 1.049 \\
\hline Final R indexes [all data] & $\mathrm{R}_{1}=0.0574, \mathrm{wR}_{2}=0.1524$ \\
\hline Largest diff. peak/hole / e $\AA^{-3}$ & $\mathrm{R}_{1}=0.0823, \mathrm{wR}_{2}=0.1789$ \\
\hline & $0.14 /-0.22$ \\
\hline
\end{tabular}

\section{Characterization of Products 3}<smiles>O=C(CC(F)F)c1ccccc1</smiles>

3,3-difluoro-1-phenylpropan-1-one (3a): The reaction was conducted on $0.2 \mathrm{mmol} \mathrm{scale}$. The product 3a (30.4 mg, 89\% yield) as colorless oil was purified by flash column chromatography (PE/DCM = 2/1); ${ }^{1} \mathrm{H}$ NMR (400 MHz, CDCl $)$ ): (ppm) $\delta 7.94(\mathrm{~d}, J=7.6 \mathrm{~Hz}, 2 \mathrm{H}), 7.63(\mathrm{t}, J=7.5 \mathrm{~Hz}, 1 \mathrm{H}), 7.50(\mathrm{t}, J$ $=7.3 \mathrm{~Hz}, 2 \mathrm{H}), 6.41(\mathrm{tt}, J=55.4 \mathrm{~Hz}, 3.8 \mathrm{~Hz}, 1 \mathrm{H}), 3.55(\mathrm{td}, J=15.2 \mathrm{~Hz}, 4.1 \mathrm{~Hz}, 2 \mathrm{H}) \cdot{ }^{13} \mathrm{C}$ NMR $(100$ $\left.\mathrm{MHz}, \mathrm{CDCl}_{3}\right)$ : (ppm) $\delta 193.8\left(\mathrm{t}, \mathrm{C}-\mathrm{F}, 3 J_{C-F}=6.7 \mathrm{~Hz}\right), 136.0,134.0,128.9,128.2,114.8\left(\mathrm{t}, \mathrm{C}-\mathrm{F}, 1 J_{C-F}=\right.$ $236.9 \mathrm{~Hz}), 43.0\left(\mathrm{t}, \mathrm{C}-\mathrm{F}, 2 J_{C-F}=23.3 \mathrm{~Hz}\right) .{ }^{19} \mathrm{~F}$ NMR $\left(376.5 \mathrm{MHz}, \mathrm{CDCl}_{3}\right)$ : (ppm) $\delta-116.8$. HRMS (ESI) $\mathrm{m} / \mathrm{z}:[\mathrm{M}+\mathrm{H}]^{+}$Calcd for $\mathrm{C}_{9} \mathrm{H}_{9} \mathrm{~F}_{2} \mathrm{O}$ 171.0616; Found 171.0619.<smiles>Cc1ccc(C(=O)CC(F)F)cc1</smiles>

3,3-difluoro-1-(p-tolyl)propan-1-one (3b): The reaction was conducted on $0.2 \mathrm{mmol}$ scale. The product $3 \mathbf{b}$ (31.6 $\mathrm{mg} 86 \%$ yield) as white powder was purified by flash column chromatography $(\mathrm{PE} / \mathrm{DCM}=2 / 1) ;{ }^{1} \mathrm{H}$ NMR $\left(600 \mathrm{MHz}, \mathrm{CDCl}_{3}\right)$ : (ppm) $\delta 7.83(\mathrm{~d}, J=8.0 \mathrm{~Hz}, 2 \mathrm{H}), 7.29(\mathrm{~d}, J=7.9 \mathrm{~Hz}$, 2H), 6.39 (tt, $J=55.6 \mathrm{~Hz}, 4.5 \mathrm{~Hz}, 1 \mathrm{H}), 3.51$ (td, $J=15.2 \mathrm{~Hz}, 4.7 \mathrm{~Hz}, 2 \mathrm{H}), 2.43(\mathrm{~s}, 3 \mathrm{H}) .{ }^{13} \mathrm{C}$ NMR $(151$ $\left.\mathrm{MHz}, \mathrm{CDCl}_{3}\right)$ : (ppm) $\delta 193.4\left(\mathrm{t}, \mathrm{C}-\mathrm{F}, 3 J_{C-F}=6.6 \mathrm{~Hz}\right), 145.0,133.7,129.5,128.3,114.9\left(\mathrm{t}, \mathrm{C}-\mathrm{F}, 1 J_{C-F}=\right.$ 
$238.6 \mathrm{~Hz}), 42.9$ (t, C-F, $\left.2 J_{C-F}=23.3 \mathrm{~Hz}\right), 21.7 .{ }^{19} \mathrm{~F}$ NMR $\left(565 \mathrm{MHz}, \mathrm{CDCl}_{3}\right):(\mathrm{ppm}) \delta$-116.8. HRMS (ESI) $\mathrm{m} / \mathrm{z}:[\mathrm{M}+\mathrm{H}]^{+}$Calcd for $\mathrm{C}_{10} \mathrm{H}_{11} \mathrm{~F}_{2} \mathrm{O}$ 185.0772; Found 185.0773.<smiles>O=C(CC(F)F)c1ccc(-c2ccccc2)cc1</smiles>

1-([1,1'-biphenyl]-4-yl)-3,3-difluoropropan-1-one (3c): The reaction was conducted on $0.2 \mathrm{mmol}$ scale. The product $3 \mathbf{c}\left(37.9 \mathrm{mg}, 77 \%\right.$ yield) as white powder ( $\left.\mathrm{mp} \mathrm{131.2-132.0}{ }^{\circ} \mathrm{C}\right)$ was purified by flash column chromatography (PE/DCM = 2/1); ${ }^{1} \mathrm{H}$ NMR $\left(400 \mathrm{MHz}, \mathrm{CDCl}_{3}\right):(\mathrm{ppm}) \delta 8.02(\mathrm{~d}, J=8.6 \mathrm{~Hz}$, $2 \mathrm{H}), 7.72(\mathrm{~d}, J=8.6 \mathrm{~Hz}, 2 \mathrm{H}), 7.65-7.62(\mathrm{~m}, 2 \mathrm{H}), 7.50-7.46(\mathrm{~m}, 2 \mathrm{H}), 7.44-7.40(\mathrm{~m}, 1 \mathrm{H}), 6.44(\mathrm{tt}, J=$ $55.5 \mathrm{~Hz}, 4.7 \mathrm{~Hz}, 1 \mathrm{H}), 3.58$ (td, $J=15.2 \mathrm{~Hz}, 4.6 \mathrm{~Hz}, 2 \mathrm{H}) .{ }^{13} \mathrm{C} \mathrm{NMR}\left(100 \mathrm{MHz}, \mathrm{CDCl}_{3}\right)$ : (ppm) $\delta 193.4$ (t, C-F, $\left.3 J_{C-F}=6.6 \mathrm{~Hz}\right), 146.7,139.5,134.7,129.1,128.8,128.5,127.5,127.3,114.9$ (t, C-F, $1 J_{C-F}=$ $237.0 \mathrm{~Hz}$ ), 43.0 (t, C-F, $\left.2 J_{C-F}=23.4 \mathrm{~Hz}\right) .{ }^{19} \mathrm{~F}$ NMR $\left(376.5 \mathrm{MHz}, \mathrm{CDCl}_{3}\right.$ ): (ppm) $\delta$-116.7. HRMS (ESI) $\mathrm{m} / \mathrm{z}:[\mathrm{M}+\mathrm{H}]^{+}$Calcd for $\mathrm{C}_{15} \mathrm{H}_{13} \mathrm{~F}_{2} \mathrm{O} 247.0929$; Found 247.0932.<smiles>COc1ccc2cc(C(=O)CC(F)F)ccc2c1</smiles>

3,3-difluoro-1-(6-methoxynaphthalen-2-yl)propan-1-one (3d): The reaction was conducted on 0.2 mmol scale. The product $3 \mathbf{d}\left(37.1 \mathrm{mg}, 74 \%\right.$ yield) as white powder $\left(\mathrm{mp} 90.4-91.8^{\circ} \mathrm{C}\right)$ was purified by flash column chromatography ( $\mathrm{PE} / \mathrm{DCM}=2 / 1) ;{ }^{1} \mathrm{H}$ NMR $\left(400 \mathrm{MHz}, \mathrm{CDCl}_{3}\right)$ : (ppm) $\delta 8.33(\mathrm{~s}, 1 \mathrm{H})$, $7.95(\mathrm{~d}, J=8.6 \mathrm{~Hz}, 1 \mathrm{H}), 7.84$ (d, $J=8.9 \mathrm{~Hz}, 1 \mathrm{H}), 7.77(\mathrm{~d}, J=8.6 \mathrm{~Hz}, 1 \mathrm{H}), 7.22(\mathrm{~d}, J=9.0 \mathrm{~Hz}, 1 \mathrm{H})$, 7.15(s, 1H), 6.46 (tt, $J=55.6 \mathrm{~Hz}, 4.2 \mathrm{~Hz}, 1 \mathrm{H}), 3.95(\mathrm{~s}, 3 \mathrm{H}), 3.63$ (td, $J=15.3 \mathrm{~Hz}, 4.3 \mathrm{~Hz}, 2 \mathrm{H}) .{ }^{13} \mathrm{C}$ NMR (100 MHz, CDCl $)$ : (ppm) $\delta 193.4\left(\mathrm{t}, \mathrm{C}-\mathrm{F}, 3 J_{C-F}=6.6 \mathrm{~Hz}\right), 160.2,137.7,131.5,131.3,130.2$, 127.7, 127.5, 124.2, 120.1, 115.1 (t, C-F, $1 J_{C-F}=236.9 \mathrm{~Hz}$ ), 105.8, 55.5, 42.9 (t, C-F, $2 J_{C-F}=23.3 \mathrm{~Hz}$ ). ${ }^{19} \mathrm{~F}$ NMR (376.5 MHz, $\mathrm{CDCl}_{3}$ ): (ppm) $\delta$-116.6. HRMS (ESI) $\mathrm{m} / \mathrm{z}$ : $[\mathrm{M}+\mathrm{H}]^{+}$Calcd for $\mathrm{C}_{14} \mathrm{H}_{13} \mathrm{~F}_{2} \mathrm{O}_{2}$ 251.0878; Found 251.0881.

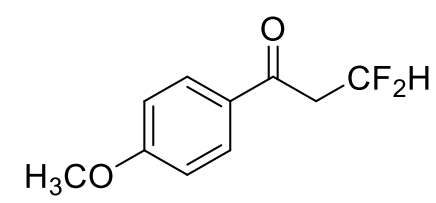

3,3-difluoro-1-(4-methoxyphenyl)propan-1-one (3e): The reaction was conducted on $0.2 \mathrm{mmol}$ scale. The product 3e (28.4 mg, $71 \%$ yield) as white powder $\left(83.5-84.5^{\circ} \mathrm{C}\right)$ was purified by flash column chromatography (PE/DCM = 2/1); ${ }^{1} \mathrm{H}$ NMR $\left(400 \mathrm{MHz}, \mathrm{CDCl}_{3}\right)$ : (ppm) $\delta 7.92(\mathrm{~d}, J=8.9 \mathrm{~Hz}, 2 \mathrm{H}), 6.96$ (d, $J=8.9 \mathrm{~Hz}, 2 \mathrm{H}), 6.39$ (tt, $J=55.6 \mathrm{~Hz}, 4.7 \mathrm{~Hz}, 1 \mathrm{H}), 3.88$ (s, 3H), 3.49 (td, $J=15.4 \mathrm{~Hz}, 4.6 \mathrm{~Hz}, 2 \mathrm{H}$ ). ${ }^{13} \mathrm{C}$ NMR $\left(100 \mathrm{MHz}, \mathrm{CDCl}_{3}\right.$ ): (ppm) $\delta 192.2\left(\mathrm{t}, \mathrm{C}-\mathrm{F}, 3 J_{\mathrm{C}-F}=6.6 \mathrm{~Hz}\right), 164.2,130.6,129.2,115.1$ (t, C-F, $\left.1 J_{C-F}=236.8 \mathrm{~Hz}\right), 114.0,55.6,42.6\left(\mathrm{t}, \mathrm{C}-\mathrm{F}, 2 J_{C-F}=23.1 \mathrm{~Hz}\right) .{ }^{19} \mathrm{~F}$ NMR $\left(376.5 \mathrm{MHz}, \mathrm{CDCl}_{3}\right):(\mathrm{ppm}) \delta$ -116.7. HRMS (ESI) m/z: [M+H] Calcd for $\mathrm{C}_{10} \mathrm{H}_{11} \mathrm{~F}_{2} \mathrm{O}_{2}$ 201.0722; Found 201.0723. 
<smiles>O=C(CC(F)(F)F)c1ccc(OCc2ccccc2)cc1</smiles>

1-(4-(benzyloxy)phenyl)-3,3-difluoropropan-1-one (3f): The reaction was conducted on $0.2 \mathrm{mmol}$ scale. The product $3 \mathbf{f}$ (38.1 mg, 69\% yield) as white powder ( $\mathrm{mp} 79.4-80.2{ }^{\circ} \mathrm{C}$ ) was purified by flash column chromatography $(\mathrm{PE} / \mathrm{DCM}=2 / 1) ;{ }^{1} \mathrm{H} \mathrm{NMR}\left(400 \mathrm{MHz}, \mathrm{CDCl}_{3}\right)$ : (ppm) $\delta 7.92(\mathrm{~d}, J=8.9 \mathrm{~Hz}$, 2H), 7.45-7.34 (m, 5H), 7.04 (d, $J=8.9 \mathrm{~Hz}, 2 \mathrm{H}), 6.39$ (tt, $J=55.6 \mathrm{~Hz}, 4.6 \mathrm{~Hz}, 1 \mathrm{H}), 5.15(\mathrm{~s}, 2 \mathrm{H}), 3.48$ $(\mathrm{td}, J=15.4 \mathrm{~Hz}, 4.7 \mathrm{~Hz}, 2 \mathrm{H}) .{ }^{13} \mathrm{C} \mathrm{NMR}\left(100 \mathrm{MHz}, \mathrm{CDCl}_{3}\right)$ : (ppm) $\delta 192.2\left(\mathrm{t}, \mathrm{C}-\mathrm{F}, 3 J_{C-F}=6.6 \mathrm{~Hz}\right)$, $163.3,135.9,130.6,129.4,128.8,128.4,127.5,115.0\left(\mathrm{t}, \mathrm{C}-\mathrm{F}, 1 J_{C-F}=236.9 \mathrm{~Hz}\right), 114.9,70.3,42.7(\mathrm{t}$, $\left.\mathrm{C}-\mathrm{F}, 2 J_{C-F}=23.2 \mathrm{~Hz}\right) .{ }^{19} \mathrm{~F}$ NMR $\left(376.5 \mathrm{MHz}, \mathrm{CDCl}_{3}\right):(\mathrm{ppm}) \delta-116.7$. HRMS (ESI) m/z: $[\mathrm{M}+\mathrm{H}]^{+}$ Calcd for $\mathrm{C}_{16} \mathrm{H}_{15} \mathrm{~F}_{2} \mathrm{O}_{2} 277.1035$, found 277.1038 .<smiles>O=C(CC(F)(F)F)c1cccc(OCc2ccccc2)c1</smiles>

1-(3-(benzyloxy)phenyl)-3,3-difluoropropan-1-one (3g): The reaction was conducted on $0.2 \mathrm{mmol}$ scale. The product $3 \mathrm{~g}$ (42.0 $\mathrm{mg}, 76 \%$ yield) as colorless oil was purified by flash column chromatography $(\mathrm{PE} / \mathrm{DCM}=2 / 1) ;{ }^{1} \mathrm{H} \mathrm{NMR}\left(400 \mathrm{MHz}, \mathrm{CDCl}_{3}\right)$ : (ppm) $\delta$ 7.56-7.55 (m, 1H), 6.39 (t, $J$ $=7.8 \mathrm{~Hz}, 1 \mathrm{H}), 7.46-7.33(\mathrm{~m}, 6 \mathrm{H}), 7.25-7.22(\mathrm{~m}, 1 \mathrm{H}), 6.40(\mathrm{tt}, J=55.5 \mathrm{~Hz}, 4.6 \mathrm{~Hz}, 1 \mathrm{H}), 5.12(\mathrm{~s}, 2 \mathrm{H})$, $3.52(\mathrm{td}, J=15.2 \mathrm{~Hz}, 4.6 \mathrm{~Hz}, 2 \mathrm{H}) .{ }^{13} \mathrm{C} \mathrm{NMR}\left(100 \mathrm{MHz}, \mathrm{CDCl}_{3}\right):(\mathrm{ppm}) \delta 193.6\left(\mathrm{t}, \mathrm{C}-\mathrm{F}, 3 J_{C-F}=6.8 \mathrm{~Hz}\right)$, $159.2,137.4,136.3,130.0,128.7,128.3,127.6,121.2,121.1,114.8$ (t, C-F, $\left.1 J_{C-F}=237.0 \mathrm{~Hz}\right), 113.5$, $70.3,43.1\left(\mathrm{t}, \mathrm{C}-\mathrm{F}, 2 J_{C-F}=23.5 \mathrm{~Hz}\right) .{ }^{19} \mathrm{~F}$ NMR (376.5 MHz, $\left.\mathrm{CDCl}_{3}\right)$ : (ppm) $\delta-116.8 . \mathrm{HRMS}(\mathrm{ESI}) \mathrm{m} / \mathrm{z}$ : $[\mathrm{M}+\mathrm{H}]^{+}$Calcd for $\mathrm{C}_{16} \mathrm{H}_{15} \mathrm{~F}_{2} \mathrm{O}_{2} 277.1035$; Found 277.1033 .<smiles>CC(=O)Oc1ccc(C(=O)CC(F)(F)F)cc1</smiles>

4-(3,3-difluoropropanoyl)phenyl acetate (3h): The reaction was conducted on $0.2 \mathrm{mmol}$ scale. The product $3 \mathrm{~h}$ (29.6 $\mathrm{mg}, 65 \%$ yield) as white powder was purified by flash column chromatography $(\mathrm{PE} / \mathrm{DCM}=2 / 1) ;{ }^{1} \mathrm{H} \mathrm{NMR}\left(400 \mathrm{MHz}, \mathrm{CDCl}_{3}\right):(\mathrm{ppm}) \delta 7.97(\mathrm{~d}, J=8.2 \mathrm{~Hz}, 2 \mathrm{H}), 7.23(\mathrm{~d}, J=8.2 \mathrm{~Hz}$, 2H), 6.39 (tt, $J=55.4 \mathrm{~Hz}, 4.5 \mathrm{~Hz}, 1 \mathrm{H}), 3.52$ (td, $J=15.1 \mathrm{~Hz}, 4.6 \mathrm{~Hz}, 2 \mathrm{H}), 2.33(\mathrm{~s}, 3 \mathrm{H}) .{ }^{13} \mathrm{C}$ NMR $(100$ $\left.\mathrm{MHz}, \mathrm{CDCl}_{3}\right):(\mathrm{ppm}) \delta 192.6\left(\mathrm{t}, \mathrm{C}-\mathrm{F}, 3 J_{C-F}=6.7 \mathrm{~Hz}\right), 168.7,155.0,133.6,129.9,122.1,114.7(\mathrm{t}, \mathrm{C}-\mathrm{F}$, $\left.1 J_{C-F}=237.0 \mathrm{~Hz}\right), 42.9\left(\mathrm{t}, \mathrm{C}-\mathrm{F}, 2 J_{C-F}=23.5 \mathrm{~Hz}\right), 21.2 .{ }^{19} \mathrm{~F} \mathrm{NMR}\left(376.5 \mathrm{MHz}, \mathrm{CDCl}_{3}\right):(\mathrm{ppm}) \delta-116.8$. HRMS (ESI) m/z: [M+H] $]^{+}$Calcd for $\mathrm{C}_{11} \mathrm{H}_{11} \mathrm{~F}_{2} \mathrm{O}_{3} 229.0761$; Found 229.0765.

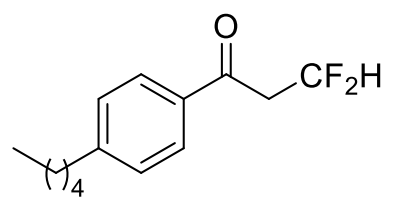

1-(4-(pentan-2-yl)phenyl)-3,3-difluoropropan-1-one (3i): The reaction was conducted on $0.2 \mathrm{mmol}$ scale. The product $3 \mathbf{i}(39.4 \mathrm{mg}, 82 \%$ yield) as white powder was purified by flash column 
chromatography (PE/DCM = 2/1); ${ }^{1} \mathrm{H}$ NMR $\left(400 \mathrm{MHz}, \mathrm{CDCl}_{3}\right)$ : (ppm) $\delta 7.85(\mathrm{~d}, J=8.0 \mathrm{~Hz}, 2 \mathrm{H}), 7.30$ $(\mathrm{d}, J=8.0 \mathrm{~Hz}, 2 \mathrm{H}), 6.40$ (tt, $J=55.6 \mathrm{~Hz}, 4.6 \mathrm{~Hz}, 1 \mathrm{H}), 3.52$ (td, $J=15.3 \mathrm{~Hz}, 4.6 \mathrm{~Hz}, 2 \mathrm{H}), 2.67$ (t, $J=$ $7.6 \mathrm{~Hz}, 2 \mathrm{H}), 1.67-1.60(\mathrm{~m}, 2 \mathrm{H}), 1.37-1.26(\mathrm{~m}, 4 \mathrm{H}), 0.89(\mathrm{t}, J=6.4 \mathrm{~Hz}, 3 \mathrm{H}) .{ }^{13} \mathrm{C}$ NMR $(100 \mathrm{MHz}$, $\left.\mathrm{CDCl}_{3}\right)$ : (ppm) $\delta 193.4\left(\mathrm{t}, \mathrm{C}-\mathrm{F}, 3 J_{C-F}=6.6 \mathrm{~Hz}\right), 150.0,133.8,128.9,128.4,115.0\left(\mathrm{t}, \mathrm{C}-\mathrm{F}, 1 J_{C-F}=236.9\right.$ $\mathrm{Hz}), 42.9$ (t, C-F, $\left.2 J_{C-F}=23.3 \mathrm{~Hz}\right), 36.0,31.4,30.7,22.5,14.0 .{ }^{19} \mathrm{~F}$ NMR $\left(376.5 \mathrm{MHz}, \mathrm{CDCl}_{3}\right):(\mathrm{ppm}) \delta$ -116.8. HRMS (ESI) m/z: [M+H] ${ }^{+}$Calcd for $\mathrm{C}_{14} \mathrm{H}_{19} \mathrm{~F}_{2} \mathrm{O}$ 241.1398; Found 241.1400.<smiles>Cc1cccc(C(=O)CC(F)(F)F)c1</smiles>

3,3-difluoro-1-(m-tolyl)propan-1-one (3j): The reaction was conducted on $0.2 \mathrm{mmol}$ scale. The product $\mathbf{3 j}$ (25.0 $\mathrm{mg}, 68 \%$ yield) as colorless oil was purified by flash column chromatography $(\mathrm{PE} / \mathrm{DCM}=2 / 1) ;{ }^{1} \mathrm{H}$ NMR $\left(400 \mathrm{MHz}, \mathrm{CDCl}_{3}\right)$ : (ppm) $\delta$ 7.75-7.72 (m, 2H), $7.43(\mathrm{~d}, J=7.5 \mathrm{~Hz}, 1 \mathrm{H})$, $7.38(\mathrm{t}, J=7.5 \mathrm{~Hz}, 1 \mathrm{H}), 6.40$ (tt, $J=55.5 \mathrm{~Hz}, J=4.6 \mathrm{~Hz}, 1 \mathrm{H}), 3.53(\mathrm{td}, J=15.2 \mathrm{~Hz}, 4.6 \mathrm{~Hz}, 2 \mathrm{H}), 2.43$ (s, 3H). ${ }^{13} \mathrm{C}$ NMR $\left(100 \mathrm{MHz}, \mathrm{CDCl}_{3}\right)$ : (ppm) $\delta 194.0\left(\mathrm{t}, \mathrm{C}-\mathrm{F}, 3 J_{\mathrm{C}-F}=6.6 \mathrm{~Hz}\right), 138.8,136.1,134.8$, 128.74, 128.66, 125.4, 114.9 (t, C-F, $1 J_{C-F}=236.9 \mathrm{~Hz}$ ), 43.0 (t, C-F, $2 J_{C-F}=23.5 \mathrm{~Hz}$ ), 21.3. ${ }^{19} \mathrm{~F}$ NMR (376.5 MHz, $\mathrm{CDCl}_{3}$ ): (ppm) $\delta$-116.9. HRMS (ESI) m/z: $[\mathrm{M}+\mathrm{H}]^{+}$Calcd for $\mathrm{C}_{10} \mathrm{H}_{11} \mathrm{~F}_{2} \mathrm{O}$ 185.0772; Found 185.0775 .<smiles>CC(C)(C)c1ccc(C(=O)CC(F)F)cc1</smiles>

1-(4-(tert-butyl)phenyl)-3,3-difluoropropan-1-one (3k): The reaction was conducted on $0.2 \mathrm{mmol}$ scale. The product $\mathbf{3 k}(37.9 \mathrm{mg}, 84 \%$ yield) as colorless oil was purified by flash column chromatography (PE/DCM = 2/1); ${ }^{1} \mathrm{H}$ NMR $\left(400 \mathrm{MHz}, \mathrm{CDCl}_{3}\right)$ : (ppm) $\delta 7.88(\mathrm{~d}, J=7.2 \mathrm{~Hz}, 2 \mathrm{H}), 7.51$ (d, $J=7.2 \mathrm{~Hz}, 2 \mathrm{H}), 6.40$ (tt, $J=55.5 \mathrm{~Hz}, 3.8 \mathrm{~Hz}, 1 \mathrm{H}), 3.52$ (td, $J=15.2 \mathrm{~Hz}, 3.7 \mathrm{~Hz}, 2 \mathrm{H}), 1.35$ (s, 9H). ${ }^{13} \mathrm{C} \mathrm{NMR}\left(100 \mathrm{MHz}, \mathrm{CDCl}_{3}\right)$ : (ppm) $\delta 193.4\left(\mathrm{t}, \mathrm{C}-\mathrm{F}, 3 J_{C-F}=6.6 \mathrm{~Hz}\right), 158.0,133.5,128.2,125.8,115.0$ (t, C-F, $\left.1 J_{C-F}=236.9 \mathrm{~Hz}\right), 42.9$ (t, C-F, $2 J_{C-F}=23.2 \mathrm{~Hz}$ ), 35.3, 31.0. ${ }^{19} \mathrm{~F}$ NMR (376.5 MHz, $\mathrm{CDCl}_{3}$ ): (ppm) $\delta$-116.8. HRMS (ESI) m/z: $[\mathrm{M}+\mathrm{H}]^{+}$Calcd for $\mathrm{C}_{13} \mathrm{H}_{17} \mathrm{~F}_{2} \mathrm{O} 227.1242$; Found 227.1245.<smiles>O=C(CC(F)F)c1ccc(Oc2ccccc2)cc1</smiles>

3,3-difluoro-1-(4-phenoxyphenyl)propan-1-one (31): The reaction was conducted on $0.2 \mathrm{mmol}$ scale. The product 31 ( $33.5 \mathrm{mg}, 64 \%$ yield) as colorless oil was purified by flash column chromatography $(\mathrm{PE} / \mathrm{DCM}=2 / 1) ;{ }^{1} \mathrm{H} \mathrm{NMR}\left(400 \mathrm{MHz}, \mathrm{CDCl}_{3}\right)$ : (ppm) $\delta 7.92(\mathrm{~d}, J=8.9 \mathrm{~Hz}, 2 \mathrm{H}), 7.42$ (t, $J=8.4 \mathrm{~Hz}$, 2H), $7.21(\mathrm{t}, J=6.6 \mathrm{~Hz}, 1 \mathrm{H}), 7.09-7.01(\mathrm{~m}, 4 \mathrm{H}), 6.39(\mathrm{tt}, J=55.6 \mathrm{~Hz}, 4.7 \mathrm{~Hz}, 1 \mathrm{H}), 3.50(\mathrm{td}, J=15.3$ $\mathrm{Hz}, 4.6 \mathrm{~Hz}, 2 \mathrm{H}) .{ }^{13} \mathrm{C}$ NMR $\left(100 \mathrm{MHz}, \mathrm{CDCl}_{3}\right):(\mathrm{ppm}) \delta 192.3$ (t, C-F, $\left.3 J_{C-F}=6.6 \mathrm{~Hz}\right), 162.9,155.1$, 130.6, 130.6 (overlap), 130.2, 125.0, 120.4, 117.3, 114.9 (t, C-F, $\left.1 J_{C-F}=238.8 \mathrm{~Hz}\right), 42.8\left(\mathrm{t}, \mathrm{C}-\mathrm{F}, 2 J_{C-F}=\right.$ $23.4 \mathrm{~Hz}) .{ }^{19} \mathrm{~F}$ NMR $\left(376.5 \mathrm{MHz}, \mathrm{CDCl}_{3}\right)$ : (ppm) $\delta$-116.7. HRMS (ESI) $\mathrm{m} / \mathrm{z}:[\mathrm{M}+\mathrm{H}]^{+}$Calcd for $\mathrm{C}_{15} \mathrm{H}_{13} \mathrm{~F}_{2} \mathrm{O}_{2}$ 263.0878; Found 263.0881. 
<smiles>COc1ccc(C(=O)CC(F)F)cc1OC</smiles>

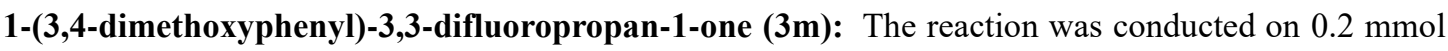
scale. The product $3 \mathrm{~m}\left(30.8 \mathrm{mg}, 67 \%\right.$ yield) as white powder $\left(\mathrm{mp} 123.8-124.2{ }^{\circ} \mathrm{C}\right)$ was purified by flash column chromatography $(\mathrm{PE} / \mathrm{DCM}=2 / 1) ;{ }^{1} \mathrm{H} \mathrm{NMR}\left(400 \mathrm{MHz}, \mathrm{CDCl}_{3}\right)$ : (ppm) $\delta$ 7.54-7.51 (m, 2H), $6.91(\mathrm{~d}, J=8.3 \mathrm{~Hz}, 1 \mathrm{H}), 6.39$ (tt, $J=55.6 \mathrm{~Hz}, 4.7 \mathrm{~Hz}, 1 \mathrm{H}), 3.96$ (s, 3H), 3.94 (s, 3H), 3.50 (td, $J=$ $15.4 \mathrm{~Hz}, 4.7 \mathrm{~Hz}, 2 \mathrm{H}) .{ }^{13} \mathrm{C}$ NMR (100 MHz, $\left.\mathrm{CDCl}_{3}\right):(\mathrm{ppm}) \delta 192.3\left(\mathrm{t}, \mathrm{C}-\mathrm{F}, 3 J_{C-F}=6.6 \mathrm{~Hz}\right), 154.1$, $149.3,129.4,123.2,115.0$ (t, C-F, $1 J_{C-F}=236.9 \mathrm{~Hz}$ ), 110.1, 109.9, 56.2, 56.0, 42.6 (t, C-F, $2 J_{C-F}=23.2$ $\mathrm{Hz}$ ). ${ }^{19} \mathrm{~F}$ NMR (376.5 MHz, $\mathrm{CDCl}_{3}$ ): (ppm) $\delta$-116.7. HRMS (ESI) m/z: $[\mathrm{M}+\mathrm{H}]^{+} \mathrm{Calcd}$ for $\mathrm{C}_{11} \mathrm{H}_{13} \mathrm{~F}_{2} \mathrm{O}_{3}$ 231.0827; Found 231.0828.<smiles>COc1ccc(C(=O)CC(F)F)c(F)c1</smiles>

3,3-difluoro-1-(2-fluoro-4-methoxyphenyl)propan-1-one (3n): The reaction was conducted on 0.2 mmol scale. The product $3 \mathbf{n}$ ( $30.7 \mathrm{mg}, 76 \%$ yield) as colorless oil was purified by flash column chromatography (PE/DCM $=2 / 1) ;{ }^{1} \mathrm{H}$ NMR $\left(400 \mathrm{MHz}, \mathrm{CDCl}_{3}\right)$ : (ppm) $\delta 7.89$ (t, $\left.J=8.8 \mathrm{~Hz}, 1 \mathrm{H}\right)$, 6.78-6.76 (m, 1H), 6.64-6.60 (m, 1H), 6.39 (tt, $J=55.5 \mathrm{~Hz}, 4.6 \mathrm{~Hz}, 1 \mathrm{H}), 3.87$ (s, 3H), 3.50 (tt, $J=15.2$ $\mathrm{Hz}, 4.0 \mathrm{~Hz}, 2 \mathrm{H}) .{ }^{13} \mathrm{C}$ NMR $\left(100 \mathrm{MHz}, \mathrm{CDCl}_{3}\right):(\mathrm{ppm}) \delta 190.2$ (td, C-F, $3 J_{C-F}=67.2 \mathrm{~Hz}, 4.0 \mathrm{~Hz}$ ), 165.7 $\left(\mathrm{d}, \mathrm{C}-\mathrm{F}, 3 J_{C-F}=12.0 \mathrm{~Hz}\right), 163.9\left(\mathrm{~d}, \mathrm{C}-\mathrm{F}, 1 J_{C-F}=253.6 \mathrm{~Hz}\right), 132.1\left(\mathrm{~d}, \mathrm{C}-\mathrm{F}, 3 J_{C-F}=2.1 \mathrm{~Hz}\right), 117.4(\mathrm{~d}, \mathrm{C}-\mathrm{F}$, $2 J_{C-F}=12.3 \mathrm{~Hz}$ ), 114.5 (td, C-F, $\left.J_{C-F}=236.3 \mathrm{~Hz}, 2.8 \mathrm{~Hz}\right), 111.2\left(\mathrm{~d}, \mathrm{C}-\mathrm{F}, 3 J_{C-F}=2.3 \mathrm{~Hz}\right), 101.7$ (d, C-F, $\left.2 J_{C-F}=27.4 \mathrm{~Hz}\right), 56.0,47.4\left(\mathrm{td}, \mathrm{C}-\mathrm{F}, J_{C-F}=23.7 \mathrm{~Hz}, 8.8 \mathrm{~Hz}\right) .{ }^{19} \mathrm{~F} \mathrm{NMR}\left(376.5 \mathrm{MHz}, \mathrm{CDCl}_{3}\right)$ : (ppm) $\delta$ -106.0, -117.5. HRMS (ESI) m/z: [M+H] ${ }^{+}$Calcd for $\mathrm{C}_{10} \mathrm{H}_{10} \mathrm{~F}_{3} \mathrm{O}_{2}$ 219.0627; Found 219.0628.<smiles>Cc1ccc(C(=O)CC(F)F)cc1C</smiles>

1-(3,4-dimethylphenyl)-3,3-difluoropropan-1-one (3o): The reaction was conducted on $0.2 \mathrm{mmol}$ scale. The product $3 \mathrm{o}(23.8 \mathrm{mg}, 60 \%$ yield $)$ as white powder was purified by flash column chromatography (PE/DCM = 2/1); ${ }^{1} \mathrm{H}$ NMR $\left(400 \mathrm{MHz}, \mathrm{CDCl}_{3}\right):(\mathrm{ppm}) \delta 7.71(\mathrm{~s}, 1 \mathrm{H}), 7.66(\mathrm{~d}, J=7.9$ $\mathrm{Hz}, 1 \mathrm{H}), 7.24$ (d, $J=7.9 \mathrm{~Hz}, 1 \mathrm{H}), 6.39$ (tt, $J=55.6 \mathrm{~Hz}, 4.4 \mathrm{~Hz}, 1 \mathrm{H}), 3.51$ (td, $J=15.3 \mathrm{~Hz}, 4.5 \mathrm{~Hz}, 2 \mathrm{H})$, $2.33(\mathrm{~s}, 6 \mathrm{H}) .{ }^{13} \mathrm{C}$ NMR $\left(100 \mathrm{MHz}, \mathrm{CDCl}_{3}\right):(\mathrm{ppm}) \delta 193.7$ (t, C-F, $\left.3 J_{C-F}=6.6 \mathrm{~Hz}\right), 143.8,137.3,134.0$, 130.1, 129.3, 126.0, 115.0 (t, C-F, $\left.1 J_{C-F}=236.8 \mathrm{~Hz}\right), 42.9$ (t, C-F, $2 J_{C-F}=23.2 \mathrm{~Hz}$ ), 20.1, $19.8 .{ }^{19} \mathrm{~F}$ NMR (376.5 MHz, $\mathrm{CDCl}_{3}$ ): (ppm) $\delta$-116.8. HRMS (ESI) m/z: $[\mathrm{M}+\mathrm{H}]^{+}$Calcd for $\mathrm{C}_{11} \mathrm{H}_{13} \mathrm{~F}_{2} \mathrm{O}$ 199.0929; Found 199.0931. 
<smiles>O=C(CC(F)F)c1ccc2c(c1)Cc1ccccc1-2</smiles>

1-(9H-fluoren-2-yl)-3,3-difluoropropan-1-one (3p): The reaction was conducted on $0.2 \mathrm{mmol}$ scale. The product 3p (26.3 mg, 51\% yield) as white powder (mp 138.5-138.8 ${ }^{\circ} \mathrm{C}$ ) was purified by flash column chromatography $(\mathrm{PE} / \mathrm{DCM}=2 / 1) ;{ }^{1} \mathrm{H} \mathrm{NMR}\left(400 \mathrm{MHz}, \mathrm{CDCl}_{3}\right)$ : (ppm) $\delta 8.09(\mathrm{~s}, 1 \mathrm{H}), 7.96(\mathrm{~d}$, $J=8.0 \mathrm{~Hz}, 1 \mathrm{H}), 7.85-7.83(\mathrm{~m}, 2 \mathrm{H}), 7.59(\mathrm{~d}, J=6.8 \mathrm{~Hz}, 1 \mathrm{H}), 7.45-7.38(\mathrm{~m}, 2 \mathrm{H}), 6.45(\mathrm{tt}, J=55.6 \mathrm{~Hz}$, $4.6 \mathrm{~Hz}, 1 \mathrm{H}), 3.94(\mathrm{~s}, 2 \mathrm{H}), 3.58(\mathrm{td}, J=15.3 \mathrm{~Hz}, 4.6 \mathrm{~Hz}, 2 \mathrm{H}) .{ }^{13} \mathrm{C} \mathrm{NMR}\left(100 \mathrm{MHz}, \mathrm{CDCl}_{3}\right):(\mathrm{ppm}) \delta$ $193.5\left(\mathrm{t}, \mathrm{C}-\mathrm{F}, 3 J_{C-F}=6.6 \mathrm{~Hz}\right), 147.4,144.6,143.6,140.2,134.4,128.4,127.6,127.2,125.3,124.8$, 121.1, 119.9, $115.1\left(\mathrm{t}, \mathrm{C}-\mathrm{F}, 1 J_{C-F}=236.9 \mathrm{~Hz}\right), 43.1\left(\mathrm{t}, \mathrm{C}-\mathrm{F}, 2 J_{C-F}=23.3 \mathrm{~Hz}\right), 36.9 .{ }^{19} \mathrm{~F}$ NMR $(376.5$ $\left.\mathrm{MHz}, \mathrm{CDCl}_{3}\right):(\mathrm{ppm}) \delta$-116.7. HRMS (ESI) m/z: $[\mathrm{M}+\mathrm{H}]^{+}$Calcd for $\mathrm{C}_{16} \mathrm{H}_{13} \mathrm{~F}_{2} \mathrm{O}$ 259.0929; Found 259.0932 .<smiles>O=C(CC(F)F)c1ccc(F)cc1</smiles>

3,3-difluoro-1-(4-fluorophenyl)propan-1-one (3q): The reaction was conducted on 0.2 mmol scale. The product 3q (17.7 $\mathrm{mg}, 47 \%$ yield) as colorless oil was purified by flash column chromatography $(\mathrm{PE} / \mathrm{DCM}=2 / 1) ;{ }^{1} \mathrm{H}$ NMR $\left(400 \mathrm{MHz}, \mathrm{CDCl}_{3}\right)$ : (ppm) $\delta$ 8.00-7.95 (m, 2H), 7.20-7.15 (m, 2H), 6.39 (tt, $J=55.5 \mathrm{~Hz}, 4.6 \mathrm{~Hz}, 1 \mathrm{H}), 3.52(\mathrm{td}, J=15.1 \mathrm{~Hz}, 4.6 \mathrm{~Hz}, 2 \mathrm{H}) .{ }^{13} \mathrm{C} \mathrm{NMR}\left(100 \mathrm{MHz}, \mathrm{CDCl}_{3}\right):(\mathrm{ppm}) \delta$ $192.2\left(\mathrm{t}, \mathrm{C}-\mathrm{F}, 3 J_{C-F}=6.8 \mathrm{~Hz}\right), 166.3\left(\mathrm{~d}, \mathrm{C}-\mathrm{F}, 1 J_{C-F}=255.0 \mathrm{~Hz}\right), 132.5,130.9\left(\mathrm{~d}, \mathrm{C}-\mathrm{F}, 3 J_{C-F}=9.4 \mathrm{~Hz}\right)$, $116.1\left(\mathrm{~d}, \mathrm{C}-\mathrm{F}, 2 J_{C-F}=22.1 \mathrm{~Hz}\right), 114.7\left(\mathrm{t}, \mathrm{C}-\mathrm{F}, 1 J_{C-F}=237.3 \mathrm{~Hz}\right), 42.9\left(\mathrm{t}, \mathrm{C}-\mathrm{F}, 2 J_{C-F}=23.4 \mathrm{~Hz}\right) .{ }^{19} \mathrm{~F}$ NMR (376.5 MHz, $\mathrm{CDCl}_{3}$ ): (ppm) $\delta-103.3$, -116.8. HRMS (ESI) m/z: $[\mathrm{M}+\mathrm{H}]^{+}$Calcd for $\mathrm{C}_{9} \mathrm{H}_{8} \mathrm{~F}_{3} \mathrm{O}$ 189.0522; Found 189.0525.<smiles>O=C(CC(F)(F)F)c1ccc(Cl)cc1</smiles>

1-(4-chlorophenyl)-3,3-difluoropropan-1-one (3r): The reaction was conducted on 0.2 mmol scale. The product $3 \mathbf{r}(22.5 \mathrm{mg}, 55 \%$ yield $)$ as colorless oil was purified by flash column chromatography $(\mathrm{PE} / \mathrm{DCM}=2 / 1) ;{ }^{1} \mathrm{H} \mathrm{NMR}\left(400 \mathrm{MHz}, \mathrm{CDCl}_{3}\right):(\mathrm{ppm}) \delta 7.88(\mathrm{~d}, J=8.7 \mathrm{~Hz}, 2 \mathrm{H}), 7.48(\mathrm{~d}, J=8.7 \mathrm{~Hz}$, $2 \mathrm{H}), 6.39(\mathrm{tt}, J=55.4 \mathrm{~Hz}, 4.6 \mathrm{~Hz}, 1 \mathrm{H}), 3.51(\mathrm{td}, J=15.1 \mathrm{~Hz}, 4.6 \mathrm{~Hz}, 2 \mathrm{H}) .{ }^{13} \mathrm{C} \mathrm{NMR}(100 \mathrm{MHz}$, $\left.\mathrm{CDCl}_{3}\right):(\mathrm{ppm}) \delta 192.6\left(\mathrm{t}, \mathrm{C}-\mathrm{F}, 3 J_{C-F}=6.6 \mathrm{~Hz}\right), 140.7,134.3,129.6,129.3,114.6\left(\mathrm{t}, \mathrm{C}-\mathrm{F}, 1 J_{C-F}=237.4\right.$ $\mathrm{Hz}$ ), 43.0 (t, C-F, $\left.2 J_{C-F}=23.6 \mathrm{~Hz}\right) .{ }^{19} \mathrm{~F}$ NMR (376.5 MHz, $\left.\mathrm{CDCl}_{3}\right)$ : (ppm) $\delta-116.8$. HRMS (ESI) m/z: $[\mathrm{M}+\mathrm{H}]^{+}$Calcd for $\mathrm{C}_{9} \mathrm{H}_{8} \mathrm{ClF}_{2} \mathrm{O} 205.0226$; Found 205.0229.<smiles>O=C(CC(F)F)c1ccc(Br)cc1</smiles> 
1-(4-bromophenyl)-3,3-difluoropropan-1-one (3s): The reaction was conducted on $0.2 \mathrm{mmol}$ scale. The product 3s (21.4 mg, 43\% yield) as white powder was purified by flash column chromatography $(\mathrm{PE} / \mathrm{DCM}=2 / 1) ;{ }^{1} \mathrm{H} \mathrm{NMR}\left(400 \mathrm{MHz}, \mathrm{CDCl}_{3}\right):(\mathrm{ppm}) \delta 7.80(\mathrm{~d}, J=8.7 \mathrm{~Hz}, 2 \mathrm{H}), 7.65(\mathrm{~d}, J=8.7 \mathrm{~Hz}$, $2 \mathrm{H}), 6.39(\mathrm{tt}, J=55.4 \mathrm{~Hz}, 4.6 \mathrm{~Hz}, 1 \mathrm{H}), 3.51(\mathrm{td}, J=15.1 \mathrm{~Hz}, 4.6 \mathrm{~Hz}, 2 \mathrm{H}) .{ }^{13} \mathrm{C} \mathrm{NMR}(100 \mathrm{MHz}$, $\left.\mathrm{CDCl}_{3}\right):(\mathrm{ppm}) \delta 192.8\left(\mathrm{t}, \mathrm{C}-\mathrm{F}, 3 J_{C-F}=6.8 \mathrm{~Hz}\right), 134.7,132.3,129.7,129.4,114.6\left(\mathrm{t}, \mathrm{C}-\mathrm{F}, 1 J_{C-F}=237.4\right.$ $\mathrm{Hz}), 42.9$ (t, C-F, $\left.2 J_{C-F}=23.4 \mathrm{~Hz}\right) .{ }^{19} \mathrm{~F}$ NMR (376.5 MHz, $\left.\mathrm{CDCl}_{3}\right)$ : (ppm) $\delta-116.8$. HRMS (ESI) m/z: $[\mathrm{M}+\mathrm{H}]^{+}$Calcd for $\mathrm{C}_{9} \mathrm{H}_{8} \mathrm{BrF}_{2} \mathrm{O} 248.9721$; Found 248.9724.<smiles>O=C1c2ccccc2CCC1C(F)(F)F</smiles>

2-(difluoromethyl)-3,4-dihydronaphthalen-1(2H)-one (3u): The reaction was conducted on 0.2 mmol scale. The product $3 \mathbf{u}(23.9 \mathrm{mg}, 61 \%$ yield) as colorless oil was purified by flash column chromatography $(\mathrm{PE} / \mathrm{DCM}=2 / 1) ;{ }^{1} \mathrm{H} \mathrm{NMR}\left(400 \mathrm{MHz}, \mathrm{CDCl}_{3}\right)$ : $(\mathrm{ppm}) \delta 8.05-8.03(\mathrm{~m}, 1 \mathrm{H}), 7.52$ (td, $J$ $=7.5 \mathrm{~Hz}, 1.4 \mathrm{~Hz}, 1 \mathrm{H}), 7.34(\mathrm{t}, J=7.5 \mathrm{~Hz}, 1 \mathrm{H}), 7.28(\mathrm{~d}, J=7.8 \mathrm{~Hz}, 1 \mathrm{H}), 6.53(\mathrm{td}, J=55.6 \mathrm{~Hz}, 2.2 \mathrm{~Hz}$, $1 \mathrm{H}), 3.13-2.90(\mathrm{~m}, 3 \mathrm{H}), 2.45-2.38(\mathrm{~m}, 1 \mathrm{H}), 2.25-2.14(\mathrm{~m}, 1 \mathrm{H}) .{ }^{13} \mathrm{C} \mathrm{NMR}\left(100 \mathrm{MHz}, \mathrm{CDCl}_{3}\right):(\mathrm{ppm}) \delta$ 194.2, 143.9, 134.1, 131.9, 128.9, 127.5, $127.0,115.4\left(\mathrm{t}, \mathrm{C}-\mathrm{F}, 1 J_{C-F}=238.8 \mathrm{~Hz}\right), 51.0\left(\mathrm{t}, \mathrm{C}-\mathrm{F}, 2 J_{C-F}=\right.$ $22.3 \mathrm{~Hz}), 28.1,20.7$ (t, C-F, $\left.3 J_{C-F}=4.2 \mathrm{~Hz}\right) .{ }^{19} \mathrm{~F} \mathrm{NMR}\left(376.5 \mathrm{MHz}, \mathrm{CDCl}_{3}\right)$ : (ppm) $\delta-127.4(\mathrm{~d}, J=8.4$ $\mathrm{Hz}$ ). HRMS (ESI) m/z: [M+H] $]^{+}$Calcd for $\mathrm{C}_{11} \mathrm{H}_{11} \mathrm{~F}_{2} \mathrm{O}$ 197.0772; Found 197.0774.<smiles>Cc1ccc(C(=O)C(C)C(F)(F)F)cc1</smiles>

3,3-difluoro-2-methyl-1-(p-tolyl)propan-1-one (3v): The reaction was conducted on $0.2 \mathrm{mmol}$ scale. The product $3 \mathbf{v}$ (24.6 mg, 62\% yield) as colorless oil was purified by flash column chromatography $(\mathrm{PE} / \mathrm{DCM}=2 / 1)$; ${ }^{1} \mathrm{H}$ NMR $\left(400 \mathrm{MHz}, \mathrm{CDCl}_{3}\right)$ : (ppm) $\delta 7.85(\mathrm{~d}, J=8.3 \mathrm{~Hz}, 2 \mathrm{H}), 7.30(\mathrm{~d}, J=8.0 \mathrm{~Hz}$, $2 \mathrm{H}), 6.23-5.94(\mathrm{~m}, 1 \mathrm{H}), 3.96-3.83(\mathrm{~m}, 1 \mathrm{H}), 2.43(\mathrm{~s}, 3 \mathrm{H}), 1.34(\mathrm{~d}, J=7.2 \mathrm{~Hz}, 3 \mathrm{H}) .{ }^{13} \mathrm{C} \mathrm{NMR}(100 \mathrm{MHz}$, $\left.\mathrm{CDCl}_{3}\right):(\mathrm{ppm}) \delta 198.2\left(\mathrm{dd}, J_{C-F}=7.3 \mathrm{~Hz}, 2.2 \mathrm{~Hz}\right), 144.9,133.1\left(\mathrm{~d}, \mathrm{C}-\mathrm{F}, 3 J_{C-F}=2.1 \mathrm{~Hz}\right), 129.6,128.6$, $117.6\left(\mathrm{t}, \mathrm{C}-\mathrm{F}, 1 J_{C-F}=240.1 \mathrm{~Hz}\right), 45.0\left(\mathrm{t}, \mathrm{C}-\mathrm{F}, 2 J_{C-F}=21.6 \mathrm{~Hz}\right), 21.7,12.4\left(\mathrm{dd}, J_{C-F}=7.0 \mathrm{~Hz}, 3.6 \mathrm{~Hz}\right)$. ${ }^{19} \mathrm{~F} \mathrm{NMR}\left(376.5 \mathrm{MHz}, \mathrm{CDCl}_{3}\right)$ : (ppm) $\delta-116.5(\mathrm{~d}, J=283.0 \mathrm{~Hz}),-126.6(\mathrm{~d}, J=283.0 \mathrm{~Hz}) . \mathrm{HRMS}(\mathrm{ESI})$ $\mathrm{m} / \mathrm{z}:[\mathrm{M}+\mathrm{H}]^{+}$Calcd for $\mathrm{C}_{11} \mathrm{H}_{13} \mathrm{~F}_{2} \mathrm{O}$ 199.0929; Found 199.0931 .<smiles>O=C(CC(F)(F)F)c1ccsc1</smiles>

3,3-difluoro-1-(thiophen-3-yl)propan-1-one (3w): The reaction was conducted on 0.2 mmol scale. The product $3 \mathbf{w}\left(17.6 \mathrm{mg}, 50 \%\right.$ yield) as white powder $\left(\mathrm{mp} 43.5-44.0{ }^{\circ} \mathrm{C}\right.$ ) was purified by flash column chromatography (PE/DCM = 2/1); ${ }^{1} \mathrm{H}$ NMR (400 MHz, $\left.\mathrm{CDCl}_{3}\right)$ : (ppm) $\delta 8.09$ (d, $\left.J=2.7 \mathrm{~Hz}, 1 \mathrm{H}\right), 7.55$ $(\mathrm{d}, J=5.1 \mathrm{~Hz}, 1 \mathrm{H}), 7.37-7.35(\mathrm{~m}, 1 \mathrm{H}), 6.36(\mathrm{tt}, J=55.5 \mathrm{~Hz}, 4.7 \mathrm{~Hz}, 1 \mathrm{H}), 3.45(\mathrm{td}, J=15.4 \mathrm{~Hz}, 4.7 \mathrm{~Hz}$, 2H). ${ }^{13} \mathrm{C}$ NMR $\left(100 \mathrm{MHz}, \mathrm{CDCl}_{3}\right)$ : (ppm) $\delta 187.8\left(\mathrm{t}, \mathrm{C}-\mathrm{F}, 3 J_{C-F}=6.8 \mathrm{~Hz}\right), 141.5,133.3,127.1,126.7$, $114.6\left(\mathrm{t}, \mathrm{C}-\mathrm{F}, 1 J_{C-F}=237.2 \mathrm{~Hz}\right), 44.2\left(\mathrm{t}, \mathrm{C}-\mathrm{F}, 2 J_{C-F}=23.2 \mathrm{~Hz}\right) .{ }^{19} \mathrm{~F}$ NMR $\left(376.5 \mathrm{MHz}, \mathrm{CDCl}_{3}\right):(\mathrm{ppm}) \delta$ 
<smiles>O=C(CC(F)F)c1cn([12F])c2ccccc12</smiles>

3,3-difluoro-1-(1-tosyl-1H-indol-3-yl)propan-1-one (3x): The reaction was conducted on $0.2 \mathrm{mmol}$ scale. The product $3 \mathbf{x}(25.4 \mathrm{mg}, 35 \%$ yield $)$ as colorless oil was purified by flash column chromatography (PE/DCM = 2/1); ${ }^{1} \mathrm{H}$ NMR $\left(400 \mathrm{MHz}, \mathrm{CDCl}_{3}\right)$ : (ppm) $\delta 8.30(\mathrm{~d}, J=7.8 \mathrm{~Hz}, 1 \mathrm{H}), 8.25$ (s, 1H), $7.93(\mathrm{~d}, J=8.2 \mathrm{~Hz}, 1 \mathrm{H}), 7.85(\mathrm{~d}, J=8.2 \mathrm{~Hz}, 2 \mathrm{H}), 7.41-7.33(\mathrm{~m}, 2 \mathrm{H}), 7.29(\mathrm{~d}, J=8.2 \mathrm{~Hz}, 2 \mathrm{H})$, $6.38(\mathrm{tt}, J=55.6 \mathrm{~Hz}, 4.7 \mathrm{~Hz}, 1 \mathrm{H}), 3.47$ (td, $J=15.5 \mathrm{~Hz}, 4.7 \mathrm{~Hz}, 2 \mathrm{H}), 2.37$ (s, 3H). ${ }^{13} \mathrm{C} \mathrm{NMR}(100 \mathrm{MHz}$, $\mathrm{CDCl}_{3}$ ): (ppm) $\delta 188.9\left(\mathrm{t}, \mathrm{C}-\mathrm{F}, 3 J_{C-F}=6.9 \mathrm{~Hz}\right), 146.3,134.9,134.2,132.8,130.4,127.2,127.1,126.2$, 125.2, 123.0, 120.9, $114.6\left(\mathrm{t}, \mathrm{C}-\mathrm{F}, 1 J_{C-F}=237.7 \mathrm{~Hz}\right), 113.2,44.5\left(\mathrm{t}, \mathrm{C}-\mathrm{F}, 2 J_{C-F}=23.1 \mathrm{~Hz}\right), 21.7 .{ }^{19} \mathrm{~F}$ NMR (376.5 MHz, CDCl $)$ : (ppm) $\delta$-116.3. HRMS (ESI) $\mathrm{m} / \mathrm{z}$ : $[\mathrm{M}+\mathrm{H}]^{+}$Calcd for $\mathrm{C}_{18} \mathrm{H}_{16} \mathrm{~F}_{2} \mathrm{NO}_{3} \mathrm{~S}$ 364.0813; Found 364.0818.<smiles>CCc1ccc(C(=O)CC(F)F)cc1</smiles>

1-(4-ethylphenyl)-3,3-difluoropropan-1-one (3z): The reaction was conducted on $8 \mathrm{mmol} \mathrm{scale.} \mathrm{The}$ product $3 \mathrm{z}$ ( $1.045 \mathrm{~g}, 66 \%$ yield) as colorless oil was purified by flash column chromatography $(\mathrm{PE} / \mathrm{DCM}=2 / 1) ;{ }^{1} \mathrm{H} \mathrm{NMR}\left(400 \mathrm{MHz}, \mathrm{CDCl}_{3}\right)$ : (ppm) $\delta 7.86(\mathrm{~d}, J=8.3 \mathrm{~Hz}, 2 \mathrm{H}), 7.31(\mathrm{~d}, J=8.2 \mathrm{~Hz}$, $2 \mathrm{H}), 6.40(\mathrm{tt}, J=55.6 \mathrm{~Hz}, 4.6 \mathrm{~Hz}, 1 \mathrm{H}), 3.52(\mathrm{td}, J=15.3 \mathrm{~Hz}, J=4.7 \mathrm{~Hz}, 2 \mathrm{H}), 2.72(\mathrm{dd}, J=15.2 \mathrm{~Hz}$, $7.6 \mathrm{~Hz}, 2 \mathrm{H}), 1.26$ (t, $J=7.6 \mathrm{~Hz}, 3 \mathrm{H}) .{ }^{13} \mathrm{C} \mathrm{NMR}\left(100 \mathrm{MHz}, \mathrm{CDCl}_{3}\right)$ : (ppm) $\delta 193.5\left(\mathrm{t}, \mathrm{C}-\mathrm{F}, 3 J_{C-F}=6.5\right.$ $\mathrm{Hz}), 151.2,133.8,128.43,128.38,115.0$ (t, C-F, $1 J_{C-F}=236.8 \mathrm{~Hz}$ ), 42.9 (t, C-F, $2 J_{C-F}=23.4 \mathrm{~Hz}$ ), 29.0, 15.1. ${ }^{19} \mathrm{~F}$ NMR (376.5 MHz, $\mathrm{CDCl}_{3}$ ): (ppm) $\delta-116.8$ HRMS (ESI) m/z: $[\mathrm{M}+\mathrm{H}]^{+}$Calcd for $\mathrm{C}_{11} \mathrm{H}_{13} \mathrm{~F}_{2} \mathrm{O}$ 199.0929; Found 199.0930.<smiles>Cc1ccc(C(O)CC(F)F)cc1</smiles>

3,3-difluoro-1-(p-tolyl)propan-1-ol (4): The reaction was conducted on $0.45 \mathrm{mmol}$ scale. The product 4 (79.6 mg, 95\% yield) as colorless oil was purified by flash column chromatography (PE/EA = 5/1); ${ }^{1} \mathrm{H}$ NMR (400 MHz, CDCl 3 ): (ppm) $\delta 7.28(\mathrm{~d}, J=7.8 \mathrm{~Hz}, 2 \mathrm{H}), 7.22(\mathrm{~d}, J=8.0 \mathrm{~Hz}, 2 \mathrm{H}), 6.15-5.85(\mathrm{~m}$, 1H), 4.92 (dd, $J=9.6 \mathrm{~Hz}, 3.9 \mathrm{~Hz}, 1 \mathrm{H}), 2.39$ (s, 3H), 2.33-2.10 (m, 2H). ${ }^{13} \mathrm{C}$ NMR (100 MHz, $\mathrm{CDCl}_{3}$ ): (ppm) $\delta 140.1,138.1,129.5,125.6,116.1\left(\mathrm{t}, \mathrm{C}-\mathrm{F}, 1 J_{C-F}=236.9 \mathrm{~Hz}\right), 69.4\left(\mathrm{dd}, J_{C-F}=7.9 \mathrm{~Hz}, 5.5 \mathrm{~Hz}\right)$, $43.1\left(\mathrm{t}, \mathrm{C}-\mathrm{F}, 2 J_{C-F}=20.6 \mathrm{~Hz}\right), 21.1 .{ }^{19} \mathrm{~F}$ NMR $\left(376.5 \mathrm{MHz}, \mathrm{CDCl}_{3}\right):(\mathrm{ppm}) \delta-116.9(\mathrm{~d}, J=286.2 \mathrm{~Hz})$, $-117.8\left(\mathrm{~d}, J=286.2 \mathrm{~Hz}\right.$ ). HRMS (ESI) $\mathrm{m} / \mathrm{z}$ : $[\mathrm{M}+\mathrm{Na}]^{+}$Calcd for $\mathrm{C}_{10} \mathrm{H}_{12} \mathrm{~F}_{2} \mathrm{ONa}$ 209.0748; Found 209.0747 . 
<smiles>COC(C#N)(CC(F)F)c1ccc(C)cc1</smiles>

4,4-difluoro-2-(p-tolyl)-2-((trimethylsilyl)oxy)butanenitrile (5): The reaction was conducted on 0.2 mmol scale. The product $5(50.9 \mathrm{mg}, 90 \%$ yield $)$ as colorless oil was purified by flash column chromatography ( $\mathrm{PE} / \mathrm{DCM}=3 / 1) ;{ }^{1} \mathrm{H}$ NMR $\left(400 \mathrm{MHz}, \mathrm{CDCl}_{3}\right)$ : (ppm) $\delta 7.42(\mathrm{~d}, J=8.1 \mathrm{~Hz}, 2 \mathrm{H}), 7.23$ (d, $J=8.0 \mathrm{~Hz}, 2 \mathrm{H}), 5.94$ (tt, $J=55.7 \mathrm{~Hz}, 4.6 \mathrm{~Hz}, 1 \mathrm{H}), 2.63-2.41(\mathrm{~m}, 2 \mathrm{H}), 2.38(\mathrm{~s}, 3 \mathrm{H}), 0.14(\mathrm{~s}, 9 \mathrm{H}) .{ }^{13} \mathrm{C}$ NMR (100 MHz, $\left.\mathrm{CDCl}_{3}\right)$ : (ppm) $\delta 139.4,136.6,129.6,124.9,119.6,114.3\left(\mathrm{t}, \mathrm{C}-\mathrm{F}, 1 J_{C-F}=238.7 \mathrm{~Hz}\right)$, $71.3\left(\mathrm{dd}, J_{C-F}=7.8 \mathrm{~Hz}, 5.9 \mathrm{~Hz}\right), 49.4\left(\mathrm{t}, \mathrm{C}-\mathrm{F}, 2 J_{C-F}=22.2 \mathrm{~Hz}\right), 21.1,0.8 .{ }^{19} \mathrm{~F}$ NMR $(376.5 \mathrm{MHz}$, $\left.\mathrm{CDCl}_{3}\right)$ : (ppm) $\delta-114.6(\mathrm{~d}, J=293.6 \mathrm{~Hz}),-115.6(\mathrm{~d}, J=293.6 \mathrm{~Hz})$. HRMS $(\mathrm{ESI}) \mathrm{m} / \mathrm{z}:[\mathrm{M}+\mathrm{Na}]^{+} \mathrm{Calcd}$ for $\mathrm{C}_{14} \mathrm{H}_{19} \mathrm{~F}_{2} \mathrm{NNaOSi} 306.1096$; Found 306.1098.<smiles>COC(CCF)(CC(N)=O)c1ccc(C)cc1</smiles>

4,4-difluoro-2-(p-tolyl)-2-((trimethylsilyl)oxy)butanamide (6): The reaction was conducted on 0.2 mmol scale. The product $6(41.0 \mathrm{mg}, 68 \%$ yield) as colorless oil was purified by flash column chromatography (PE/EA = 2/1); ${ }^{1} \mathrm{H}$ NMR $\left(400 \mathrm{MHz}, \mathrm{CDCl}_{3}\right)$ : (ppm) $\delta 7.30(\mathrm{~d}, J=8.2 \mathrm{~Hz}, 2 \mathrm{H}), 7.17$ (d, $J=8.0 \mathrm{~Hz}, 2 \mathrm{H}), 7.00(\mathrm{~s}, 1 \mathrm{H}), 6.12-5.82(\mathrm{~m}, 2 \mathrm{H}), 3.10-2.98(\mathrm{~m}, 1 \mathrm{H}), 2.76-2.63(\mathrm{~m}, 1 \mathrm{H}), 2.34(\mathrm{~s}, 3 \mathrm{H})$, -0.02 (s, 9H). ${ }^{13} \mathrm{C} \mathrm{NMR}\left(100 \mathrm{MHz}, \mathrm{CDCl}_{3}\right)$ : (ppm) $\delta 176.1,138.7,137.8,129.3,125.9,124.8,115.9$ (t, $\left.\mathrm{C}-\mathrm{F}, 1 J_{C-F}=237.7 \mathrm{~Hz}\right), 41.2\left(\mathrm{t}, \mathrm{C}-\mathrm{F}, 2 J_{C-F}=21.6 \mathrm{~Hz}\right), 21.1,1.7 .{ }^{19} \mathrm{~F} \mathrm{NMR}\left(376.5 \mathrm{MHz}, \mathrm{CDCl}_{3}\right)$ : (ppm) $\delta-112.6,-112.9$.<smiles>COc1ccc2cc(C(=O)/C=C/Nc3ccccc3)ccc2c1</smiles>

(E)-1-(6-methoxynaphthalen-2-yl)-3-(phenylamino)prop-2-en-1-one (7): The reaction was conducted on $0.2 \mathrm{mmol}$ scale. The product 7 (47.9 mg, 79\% yield) as yellow solid was purified by flash column chromatography ( $\mathrm{PE} / \mathrm{DCM}=1 / 1) ;{ }^{1} \mathrm{H}$ NMR $\left(400 \mathrm{MHz}, \mathrm{CDCl}_{3}\right)$ : (ppm) $\delta 12.20$ (d, J=12.2 Hz, $1 \mathrm{H}), 8.39(\mathrm{~s}, 1 \mathrm{H}), 8.03-8.00(\mathrm{~m}, 1 \mathrm{H}), 7.85(\mathrm{~d}, J=8.8 \mathrm{~Hz}, 1 \mathrm{H}), 7.79(\mathrm{~d}, J=8.6 \mathrm{~Hz}, 1 \mathrm{H}), 7.56$ (dd, $J=$ $12.3 \mathrm{~Hz}, 7.8 \mathrm{~Hz}, 1 \mathrm{H}), 7.36$ (t, $J=8.0 \mathrm{~Hz}, 2 \mathrm{H}), 7.20-7.07$ (m, $5 \mathrm{H}), 6.17$ (d, $J=7.9 \mathrm{~Hz}, 1 \mathrm{H}), 3.95$ (s, 3H). ${ }^{13} \mathrm{C}$ NMR (100 MHz, $\mathrm{CDCl}_{3}$ ): (ppm) $\delta 190.8,159.2,144.6,140.3,136.5,134.5,131.0,129.8,128.2$, 128.1 127.0, 124.6, 123.6, 119.5, 116.3, 105.6, 93.9, 55.4. HRMS (ESI) $\mathrm{m} / \mathrm{z}:[\mathrm{M}+\mathrm{H}]^{+}$Calcd for $\mathrm{C}_{20} \mathrm{H}_{17} \mathrm{NO}_{2} \mathrm{Na} 326.1151$; Found 326.1153 . 


\section{0. ${ }^{1} \mathrm{H}$ NMR, ${ }^{13} \mathrm{C}$ NMR, ${ }^{19}$ F NMR Spectra, and HRMS}

3,3-difluoro-1-phenylpropan-1-one (3a): ${ }^{1} \mathrm{H} \mathrm{NMR}\left(400 \mathrm{MHz}, \mathrm{CDCl}_{3}\right)$

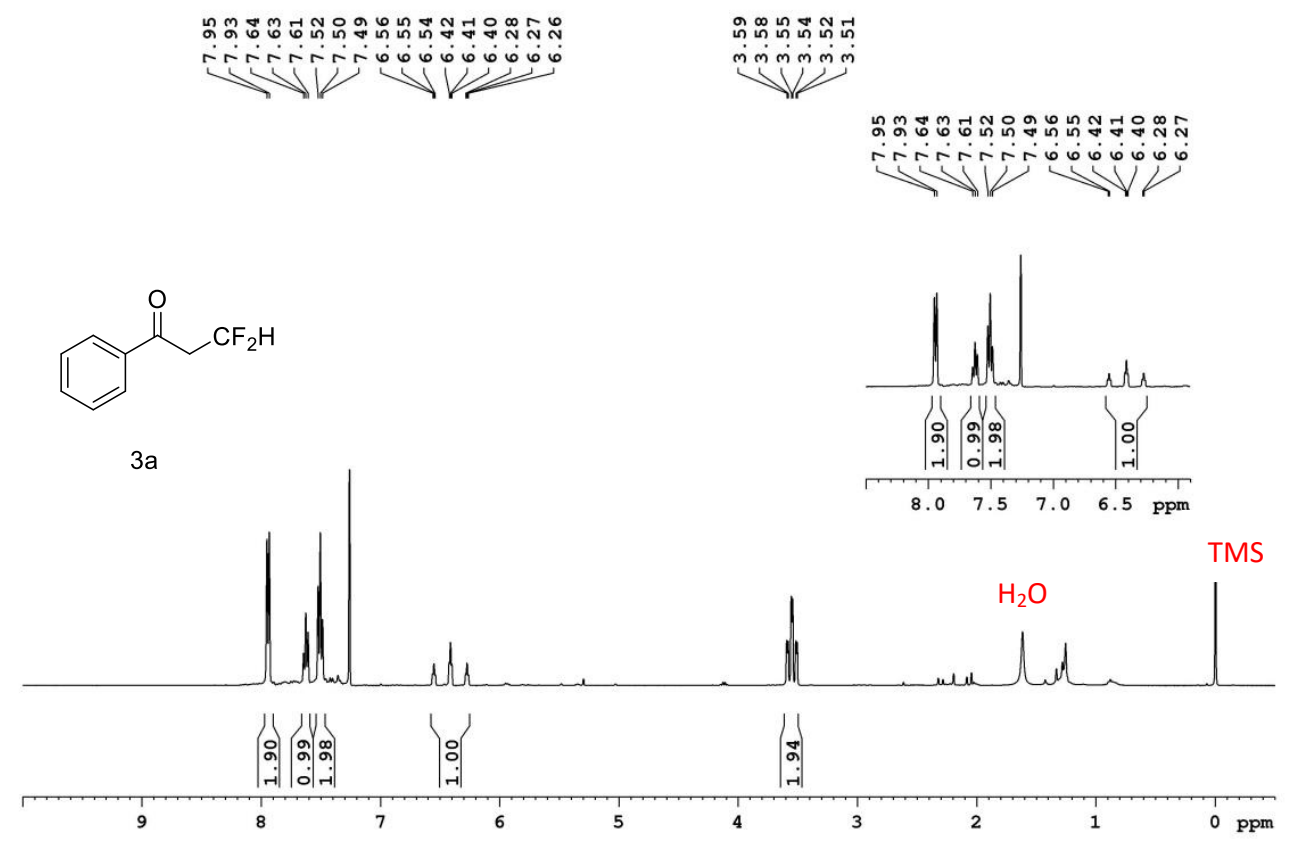

3,3-difluoro-1-phenylpropan-1-one (3a): ${ }^{13} \mathrm{C} \mathrm{NMR}\left(100 \mathrm{MHz}, \mathrm{CDCl}_{3}\right)$

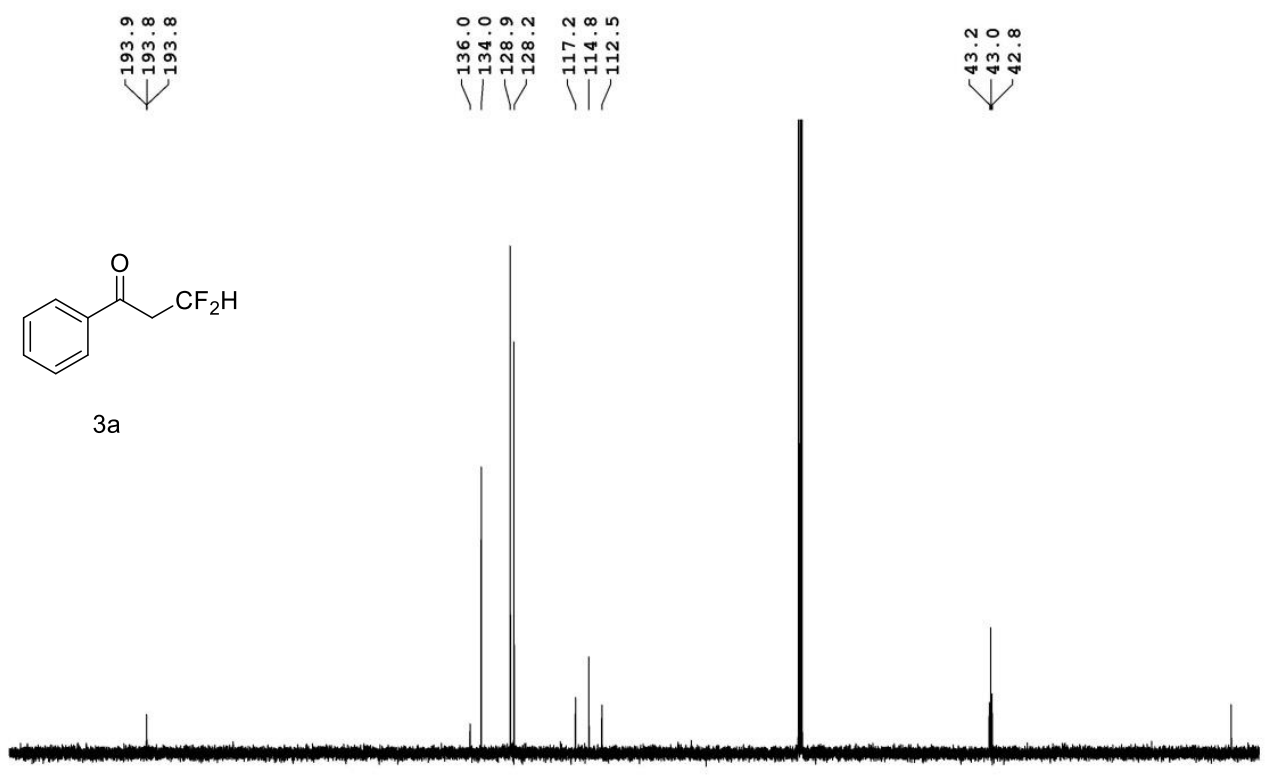

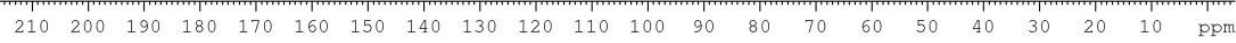


3,3-difluoro-1-phenylpropan-1-one (3a): ${ }^{19} \mathrm{~F} \mathrm{NMR} \mathrm{(376.5} \mathrm{MHz,} \mathrm{CDCl}_{3}$ )

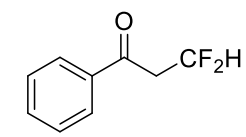

$3 a$

3,3-difluoro-1-(p-tolyl)propan-1-one (3b): ${ }^{1} \mathrm{H}$ NMR $\left(600 \mathrm{MHz}, \mathrm{CDCl}_{3}\right)$

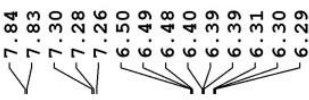

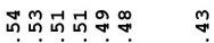
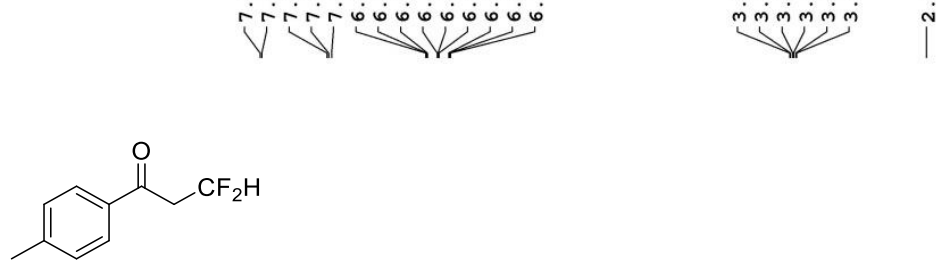

$3 b$

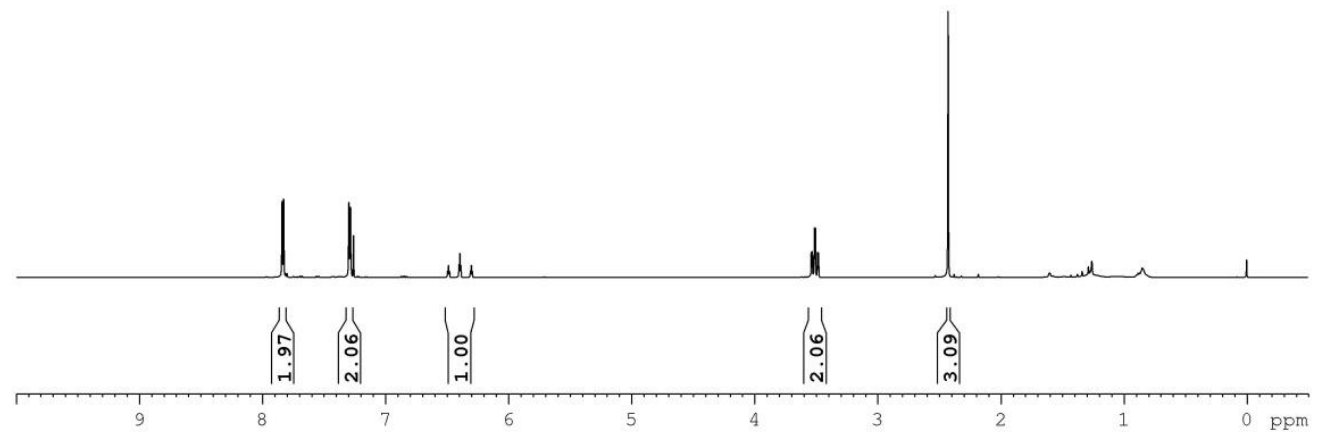


3,3-difluoro-1-(p-tolyl)propan-1-one (3b): ${ }^{13} \mathrm{C} \mathrm{NMR}\left(151 \mathrm{MHz}, \mathrm{CDCl}_{3}\right)$

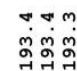

竞
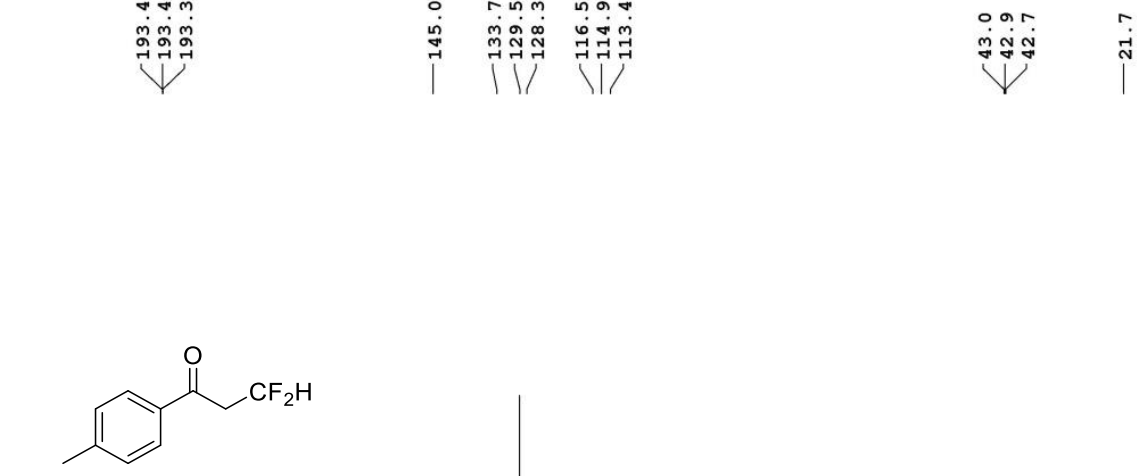

$3 b$

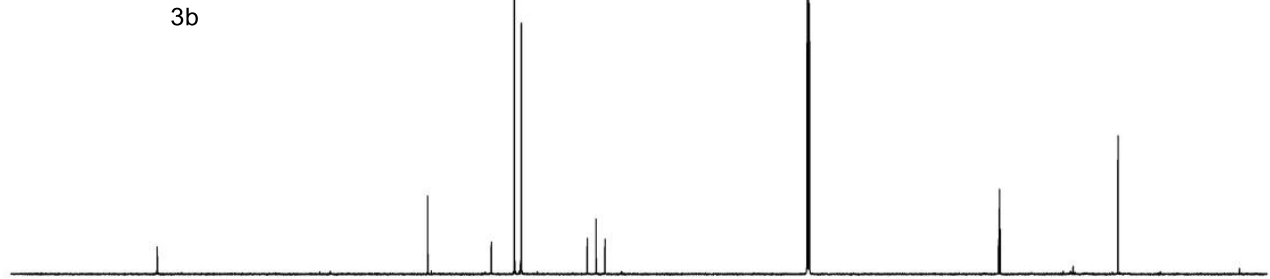

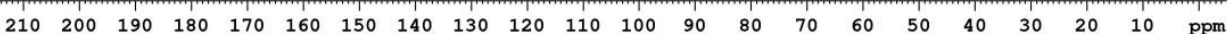

3,3-difluoro-1-(p-tolyl)propan-1-one (3b): ${ }^{19} \mathrm{~F}$ NMR (565 MHz, $\mathrm{CDCl}_{3}$ )

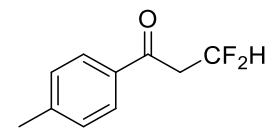

$3 b$ 


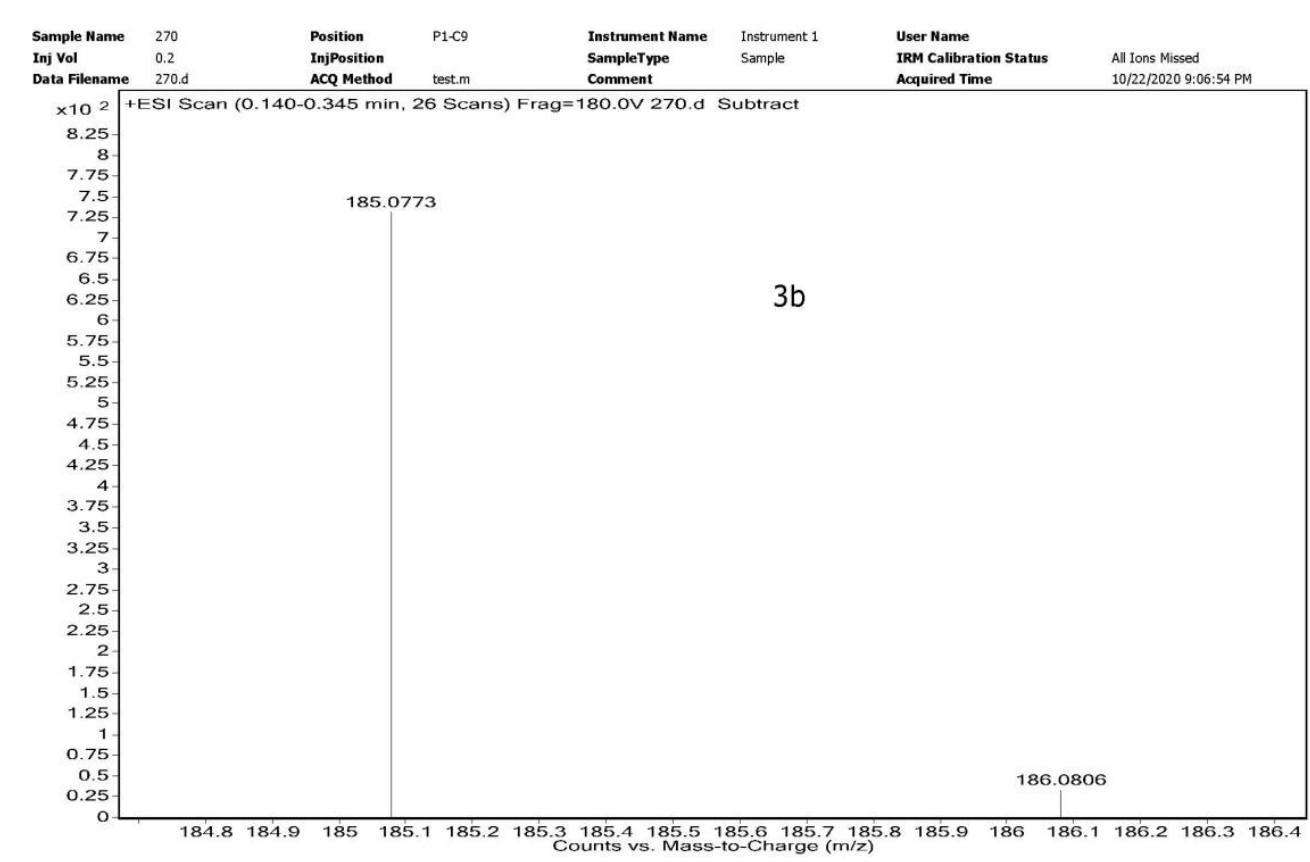

1-([1,1'-biphenyl]-4-yl)-3,3-difluoropropan-1-one (3c): ${ }^{1} \mathrm{H}$ NMR (400 MHz, $\left.\mathrm{CDCl}_{3}\right)$

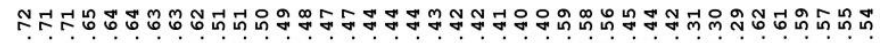

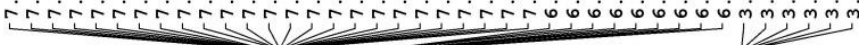<smiles>O=C(CC(F)F)c1ccc(-c2ccccc2)cc1</smiles>

$3 c$

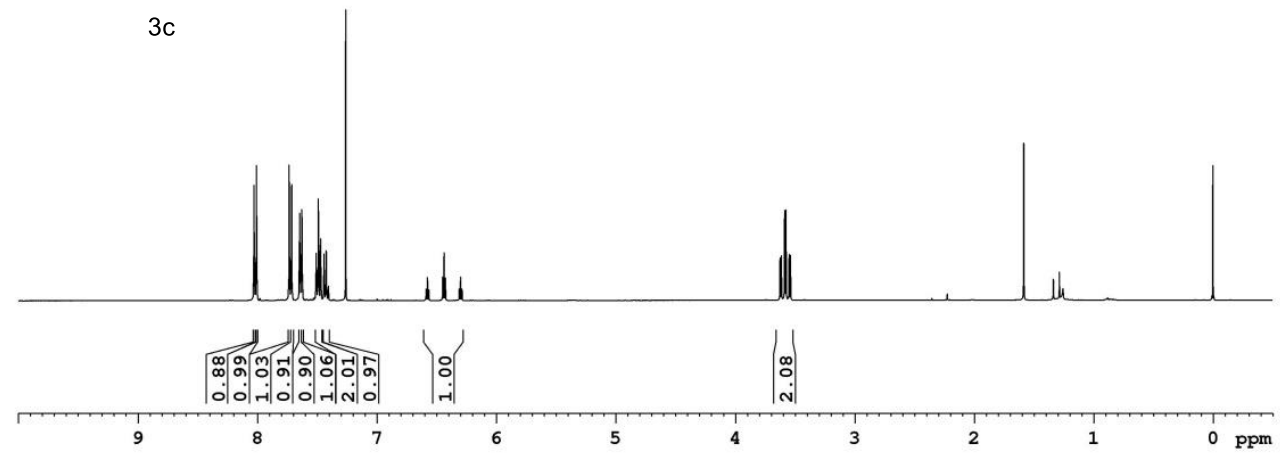


1-([1,1'-biphenyl]-4-yl)-3,3-difluoropropan-1-one (3c): ${ }^{13} \mathrm{C}$ NMR $\left(100 \mathrm{MHz}, \mathrm{CDCl}_{3}\right)$

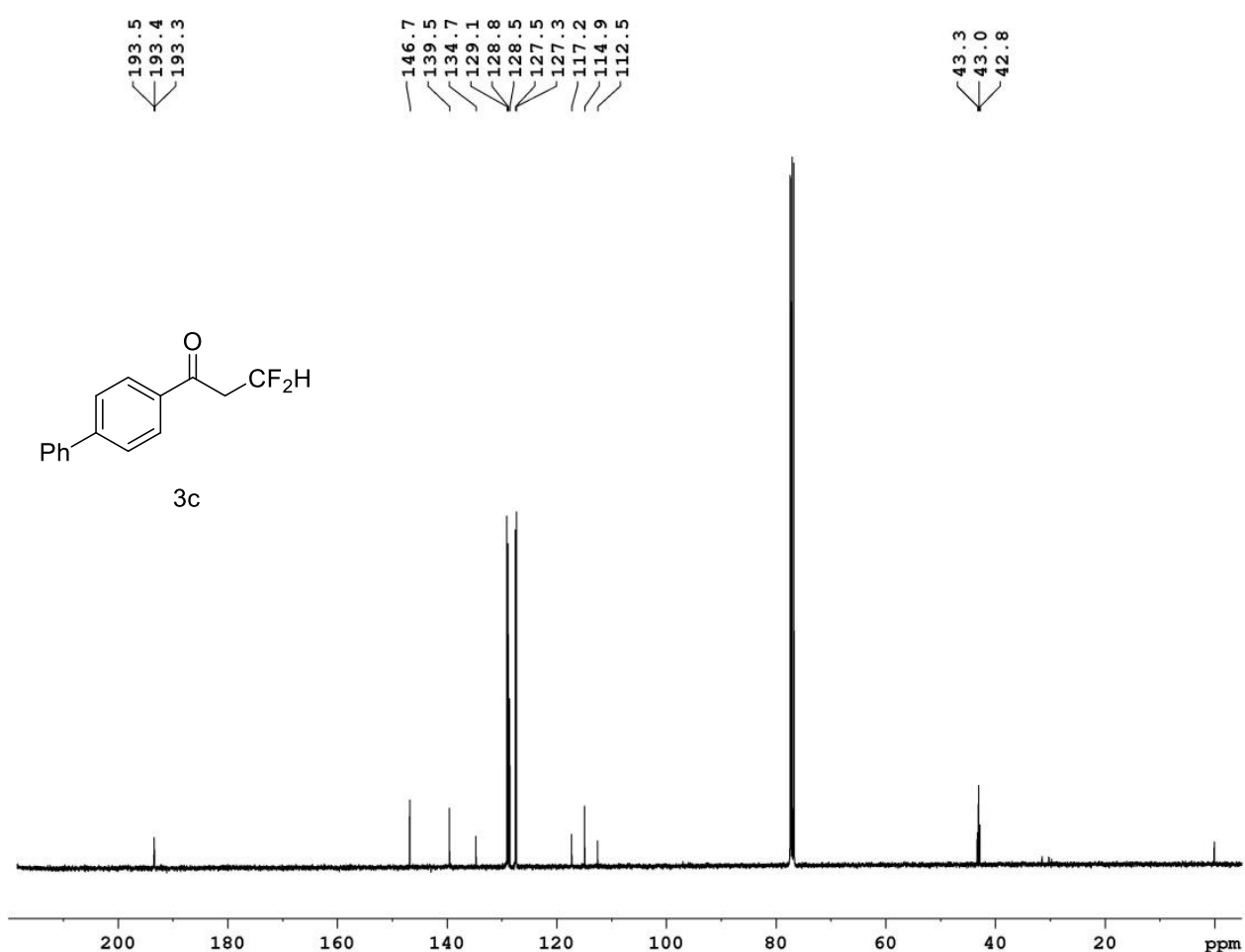

1-([1,1'-biphenyl]-4-yl)-3,3-difluoropropan-1-one (3c): ${ }^{19} \mathrm{~F} \mathrm{NMR}\left(376.5 \mathrm{MHz}, \mathrm{CDCl}_{3}\right)$<smiles>O=C(CC(F)F)c1ccc(-c2ccccc2)cc1</smiles>

$3 c$

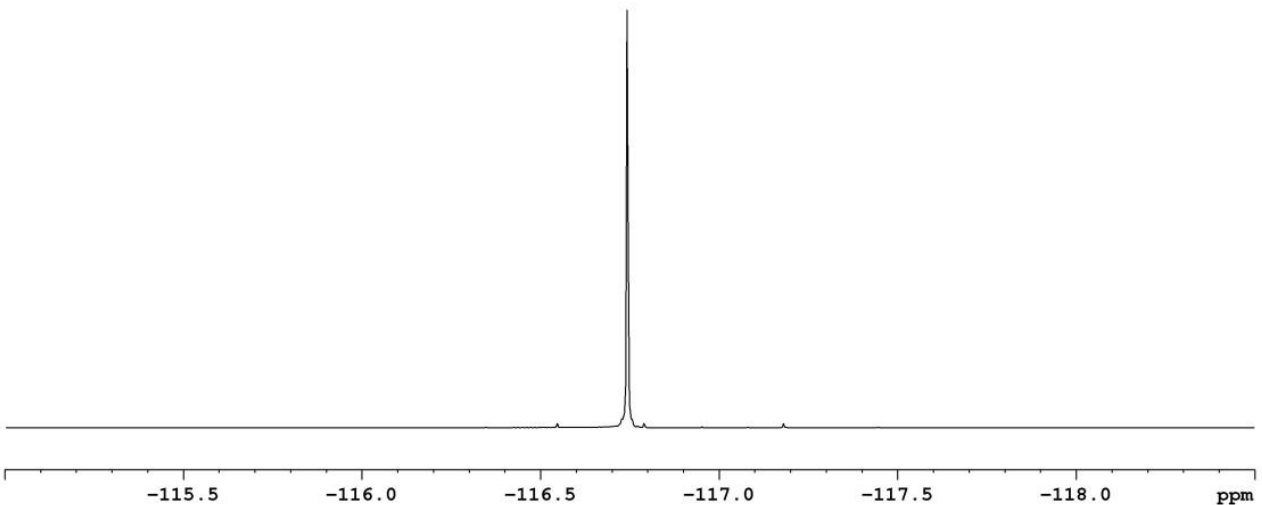


3,3-difluoro-1-(6-methoxynaphthalen-2-yl)propan-1-one (3d): ${ }^{1} \mathrm{H} \mathrm{NMR}\left(400 \mathrm{MHz}, \mathrm{CDCl}_{3}\right)$

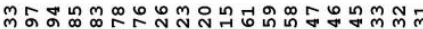

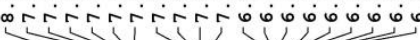

น็ํํํำํำ

imimim<smiles>COc1ccc(C(=O)CC(F)F)cc1</smiles>

$3 e$
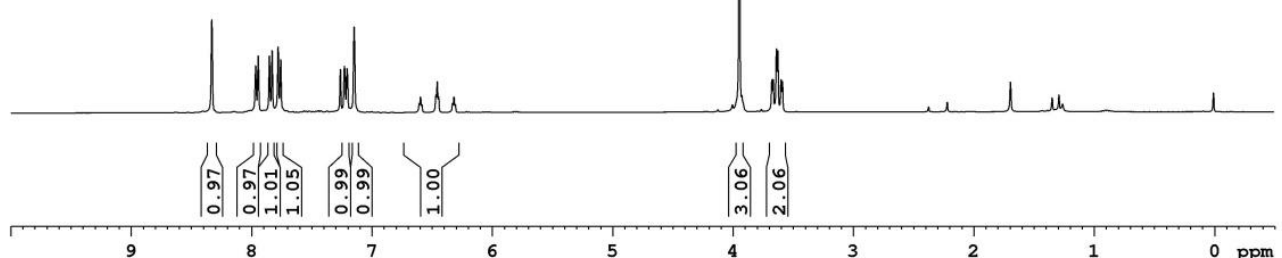

3,3-difluoro-1-(6-methoxynaphthalen-2-yl)propan-1-one (3d): ${ }^{13} \mathrm{C} \mathrm{NMR}\left(100 \mathrm{MHz}, \mathrm{CDCl}_{3}\right)$
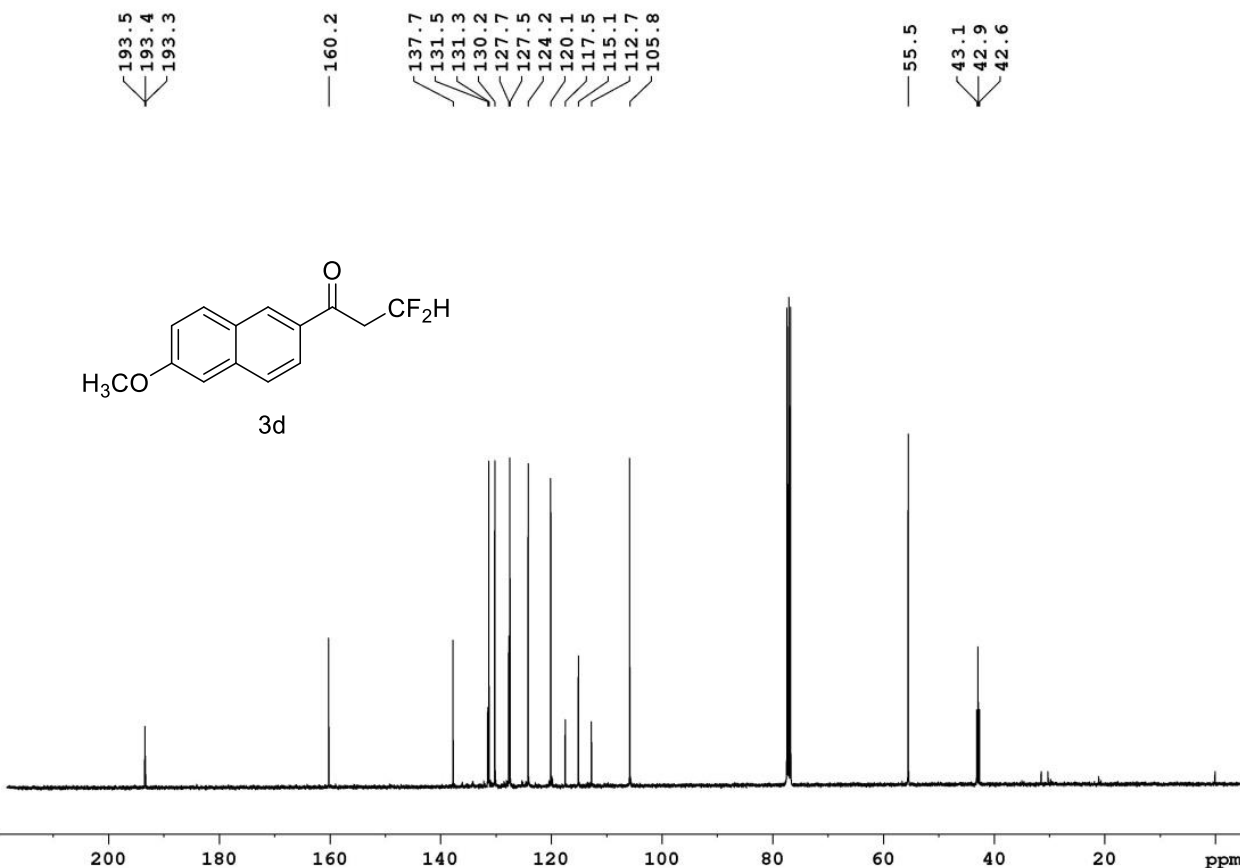
3,3-difluoro-1-(6-methoxynaphthalen-2-yl)propan-1-one (3d): ${ }^{19} \mathrm{~F}$ NMR (376.5 $\left.\mathrm{MHz}, \mathrm{CDCl}_{3}\right)$<smiles>COc1ccc2cc(C(=O)CC(F)F)ccc2c1</smiles>

$3 d$

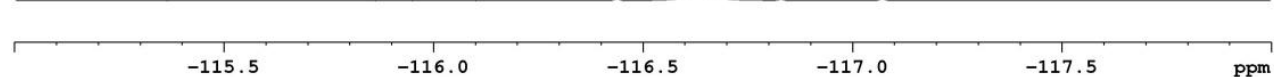

3,3-difluoro-1-(4-methoxyphenyl)propan-1-one (3e): ${ }^{1} \mathrm{H}$ NMR (400 $\left.\mathrm{MHz}, \mathrm{CDCl}_{3}\right)$

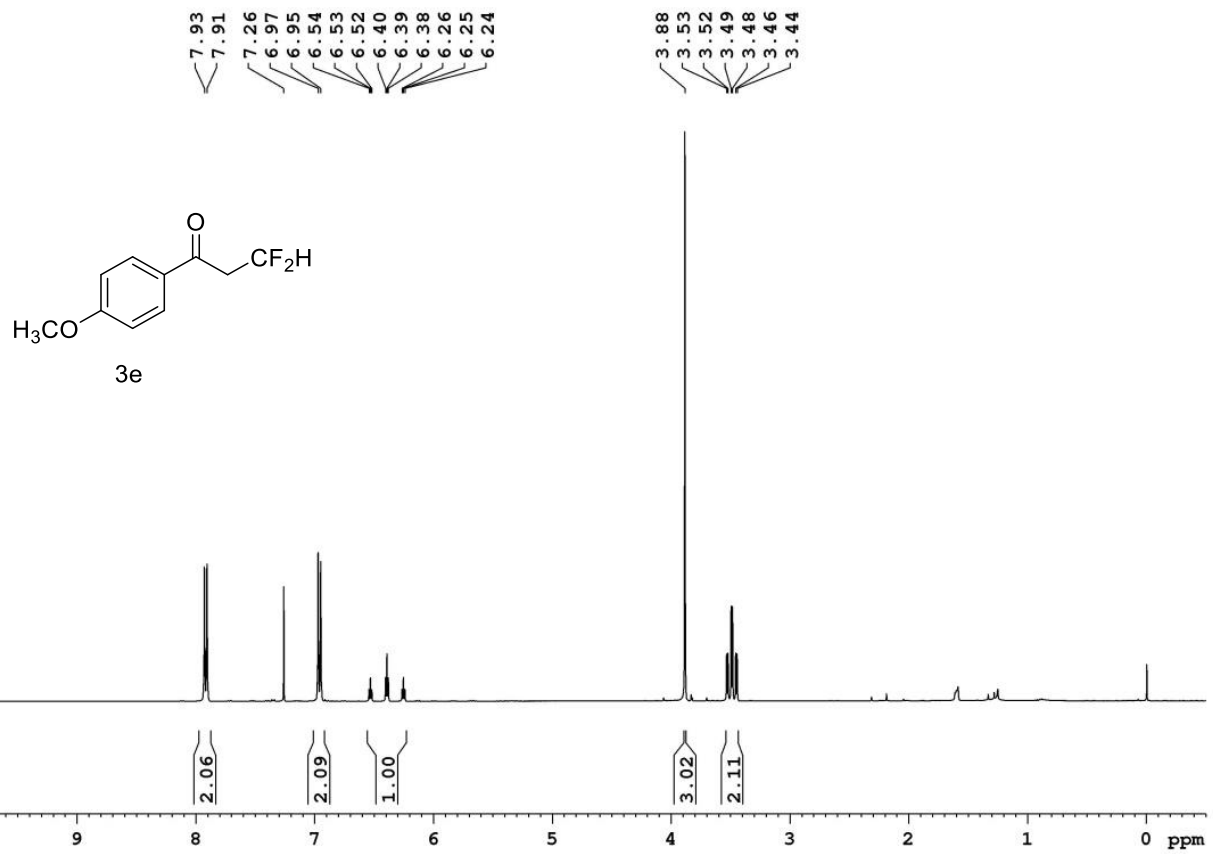


3,3-difluoro-1-(4-methoxyphenyl)propan-1-one (3e): ${ }^{13} \mathrm{C} \mathrm{NMR}\left(100 \mathrm{MHz}, \mathrm{CDCl}_{3}\right)$
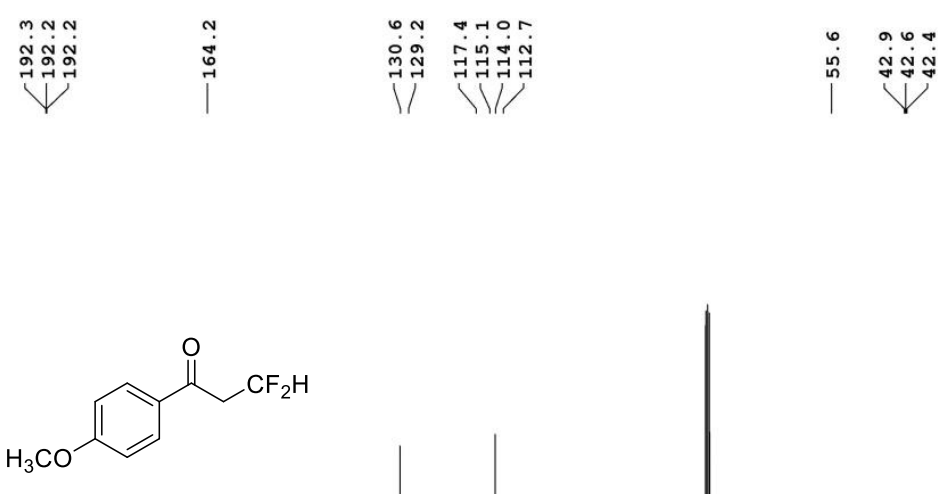

$3 e$

等

กู่

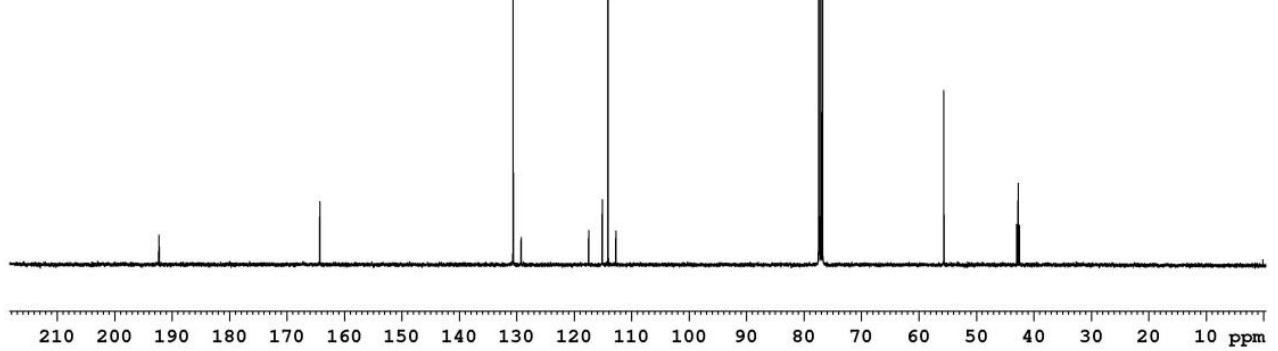

3,3-difluoro-1-(4-methoxyphenyl)propan-1-one (3e): ${ }^{19} \mathrm{~F}$ NMR (376.5 $\left.\mathrm{MHz}, \mathrm{CDCl}_{3}\right)$

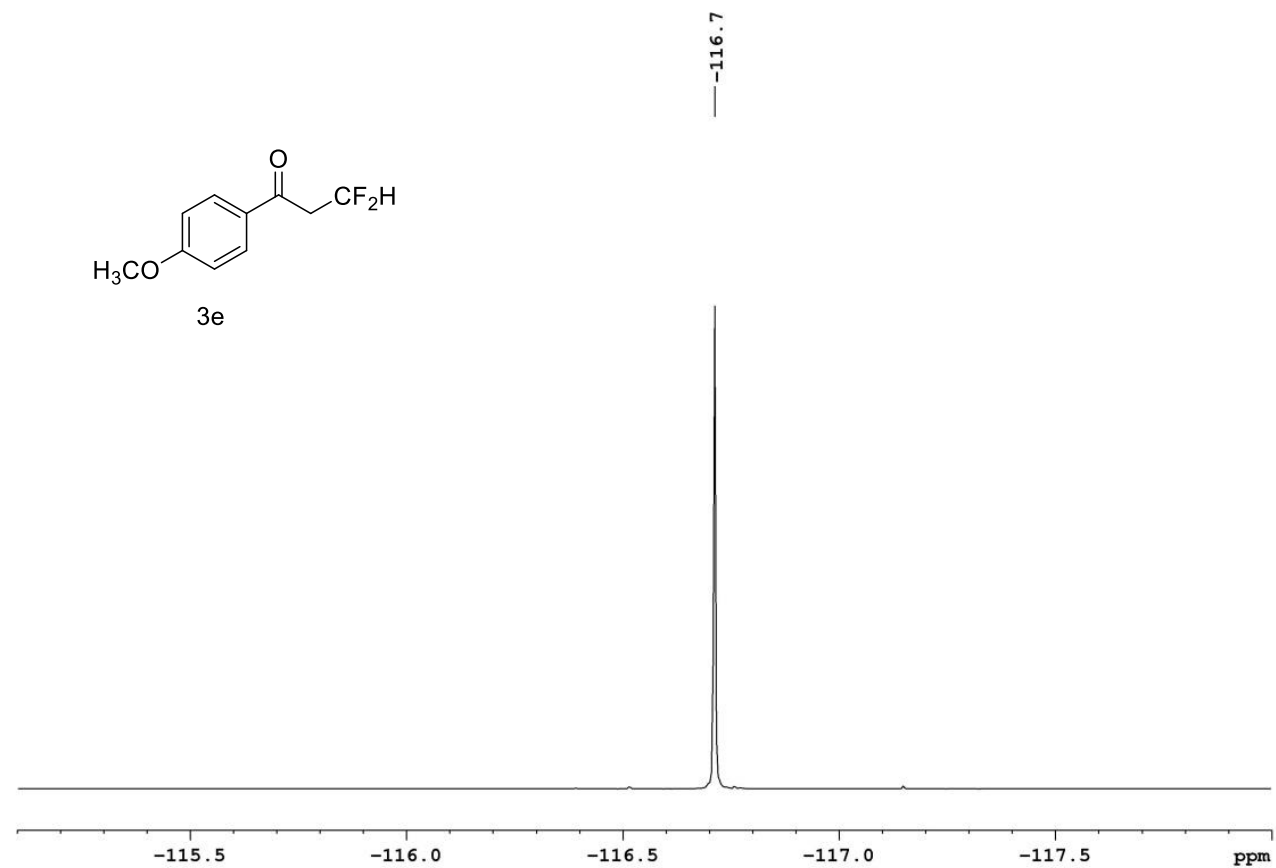


3,3-difluoro-1-(4-methoxyphenyl)propan-1-one (3e): (HRMS)

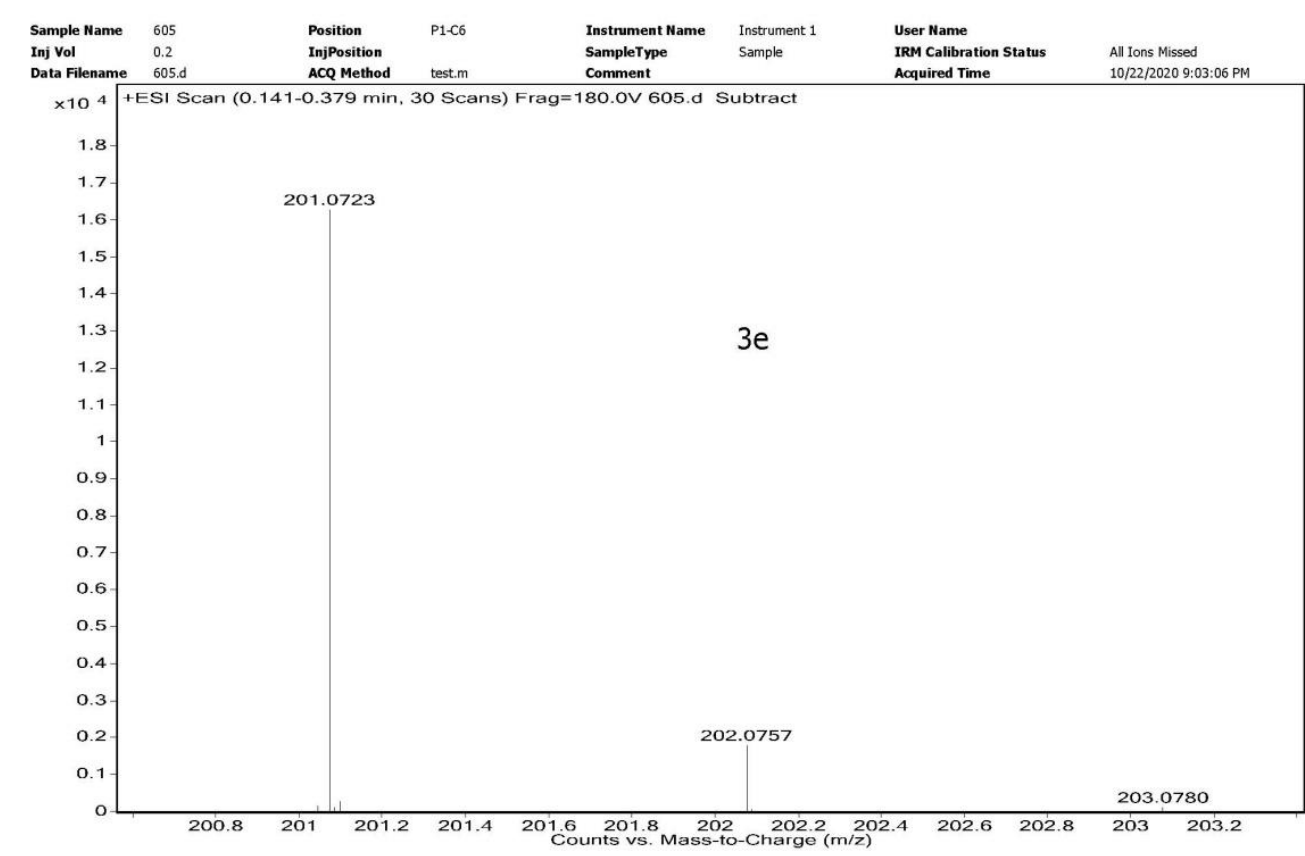

1-(4-(benzyloxy)phenyl)-3,3-difluoropropan-1-one (3f): ${ }^{1} \mathrm{H} \mathrm{NMR}\left(400 \mathrm{MHz}, \mathrm{CDCl}_{3}\right)$

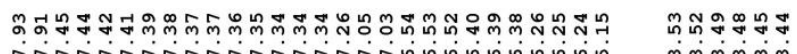

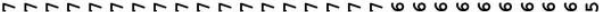

促<smiles>O=C(CC(F)F)c1ccc(OCc2ccccc2)cc1</smiles>

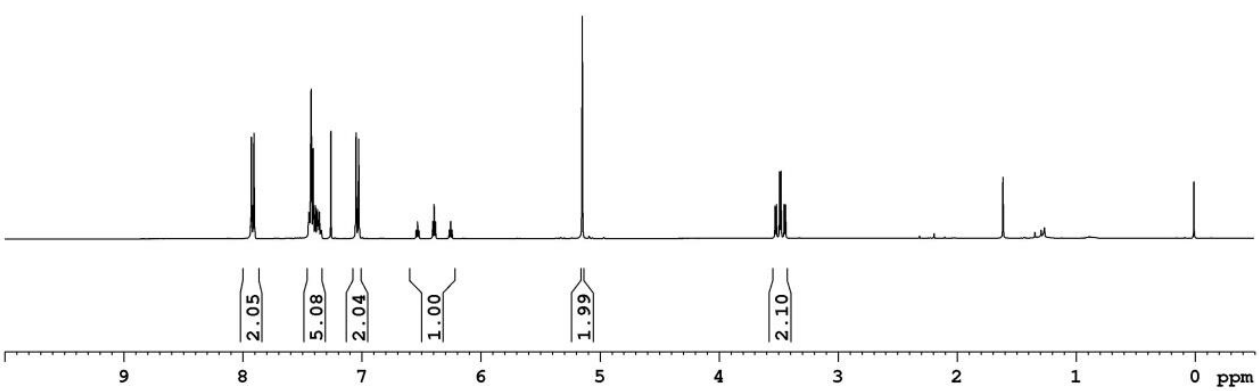


1-(4-(benzyloxy)phenyl)-3,3-difluoropropan-1-one (3f): $\left.{ }^{13} \mathrm{C} \mathrm{NMR} \mathrm{(100} \mathrm{MHz}, \mathrm{CDCl}_{3}\right)$
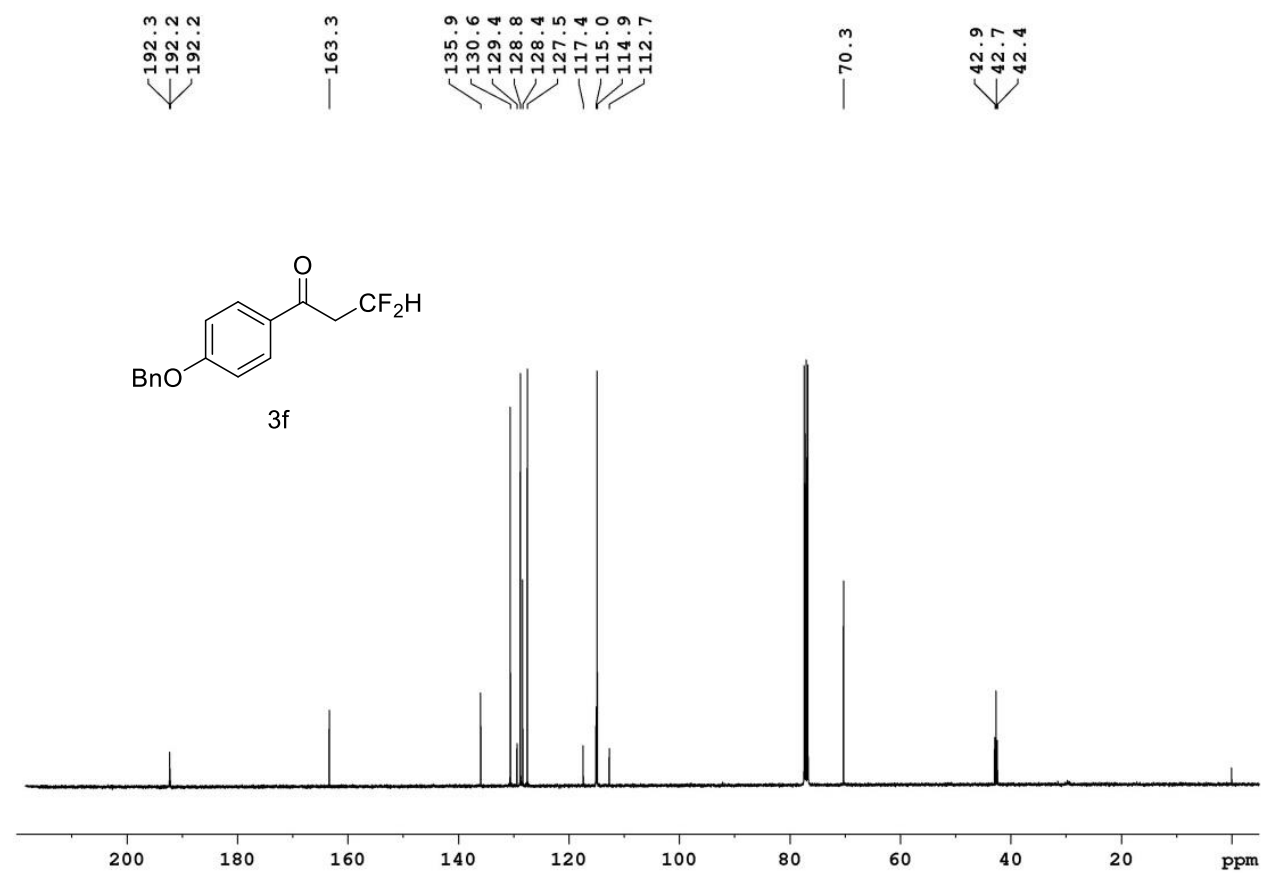

1-(4-(benzyloxy)phenyl)-3,3-difluoropropan-1-one (3f): ${ }^{19} \mathrm{~F}$ NMR (376.5 MHz, $\left.\mathrm{CDCl}_{3}\right)$<smiles>O=C(CC(F)F)c1ccc(OC(=O)c2ccccc2)cc1</smiles>

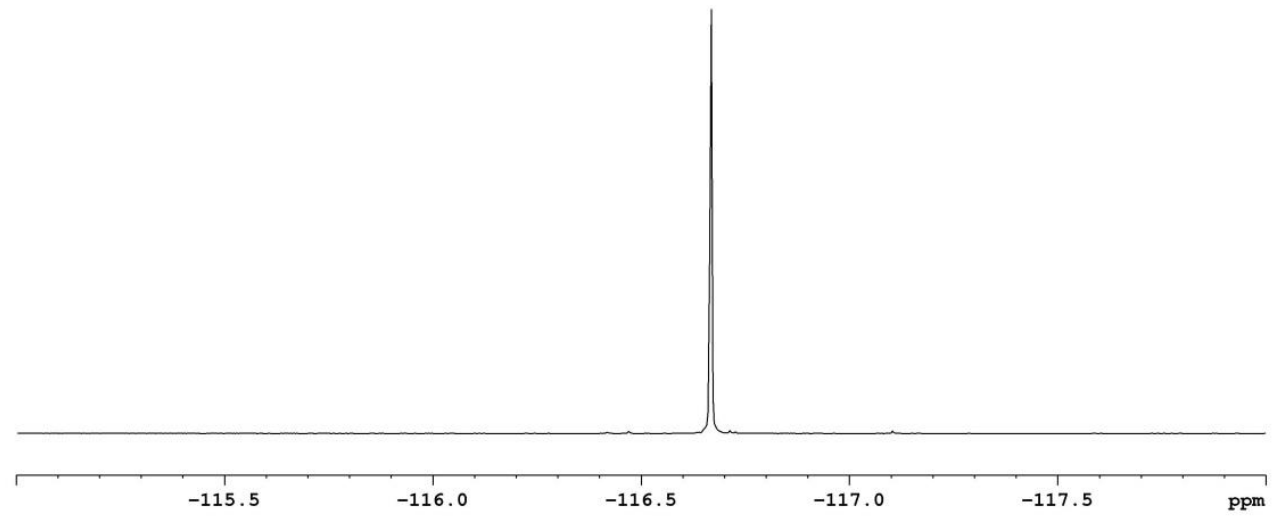


1-(3-(benzyloxy)phenyl)-3,3-difluoropropan-1-one (3g): ${ }^{1} \mathrm{H} \mathrm{NMR}\left(400 \mathrm{MHz}, \mathrm{CDCl}_{3}\right)$

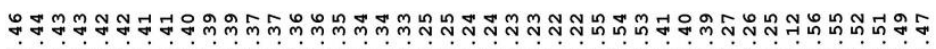

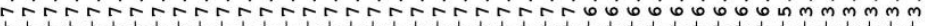<smiles>O=C(CC(F)F)c1cccc(OCc2ccccc2)c1</smiles>

$3 g$

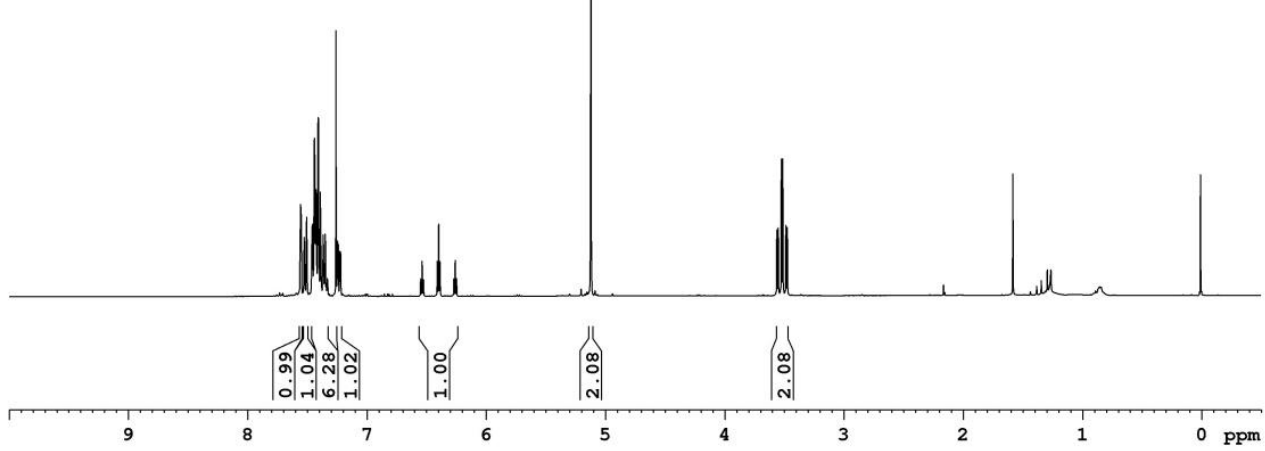

1-(3-(benzyloxy)phenyl)-3,3-difluoropropan-1-one (3g): $\left.{ }^{13} \mathrm{C} \mathrm{NMR} \mathrm{(100} \mathrm{MHz}, \mathrm{CDCl}_{3}\right)$

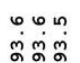

วัติ

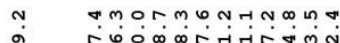

ดी

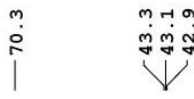<smiles>O=C(CC(F)F)c1cccc(OCc2ccccc2)c1</smiles>

$3 \mathrm{~g}$

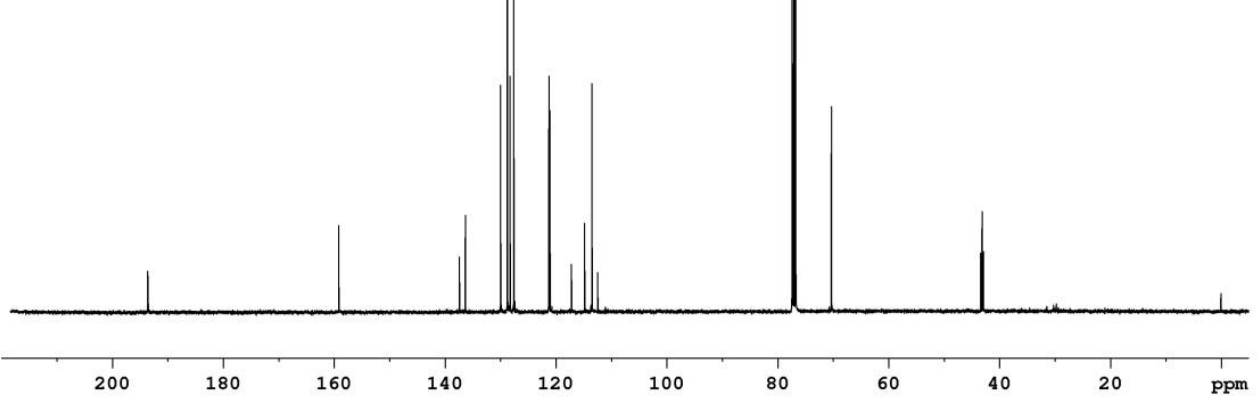


1-(3-(benzyloxy)phenyl)-3,3-difluoropropan-1-one (3g): ${ }^{19} \mathrm{~F}$ NMR (376.5 MHz, $\left.\mathrm{CDCl}_{3}\right)$<smiles>[Te]=CI</smiles><smiles>O=C(CC(F)F)c1cccc(OCc2ccccc2)c1</smiles>

$3 \mathrm{~g}$

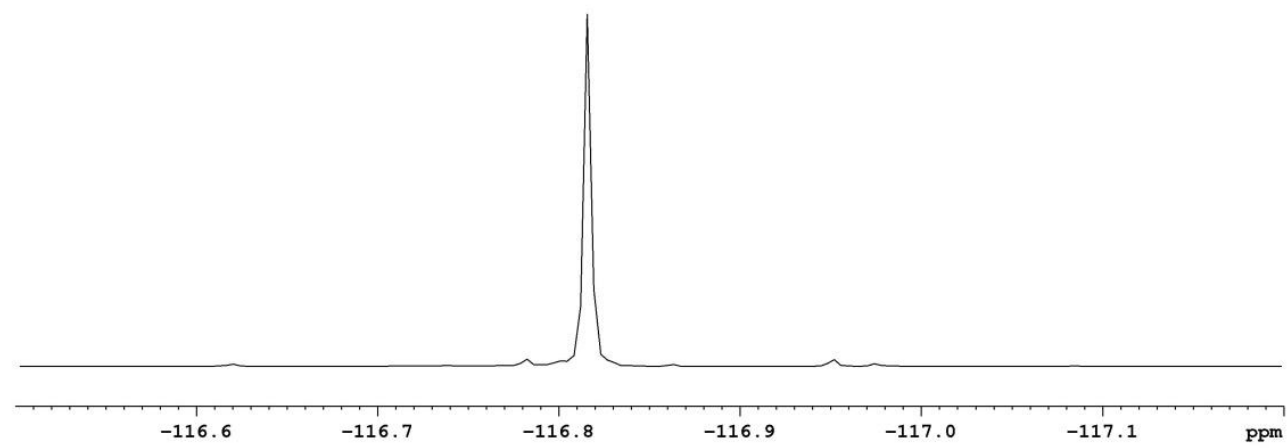

1-(3-(benzyloxy)phenyl)-3,3-difluoropropan-1-one (3g): (HRMS)

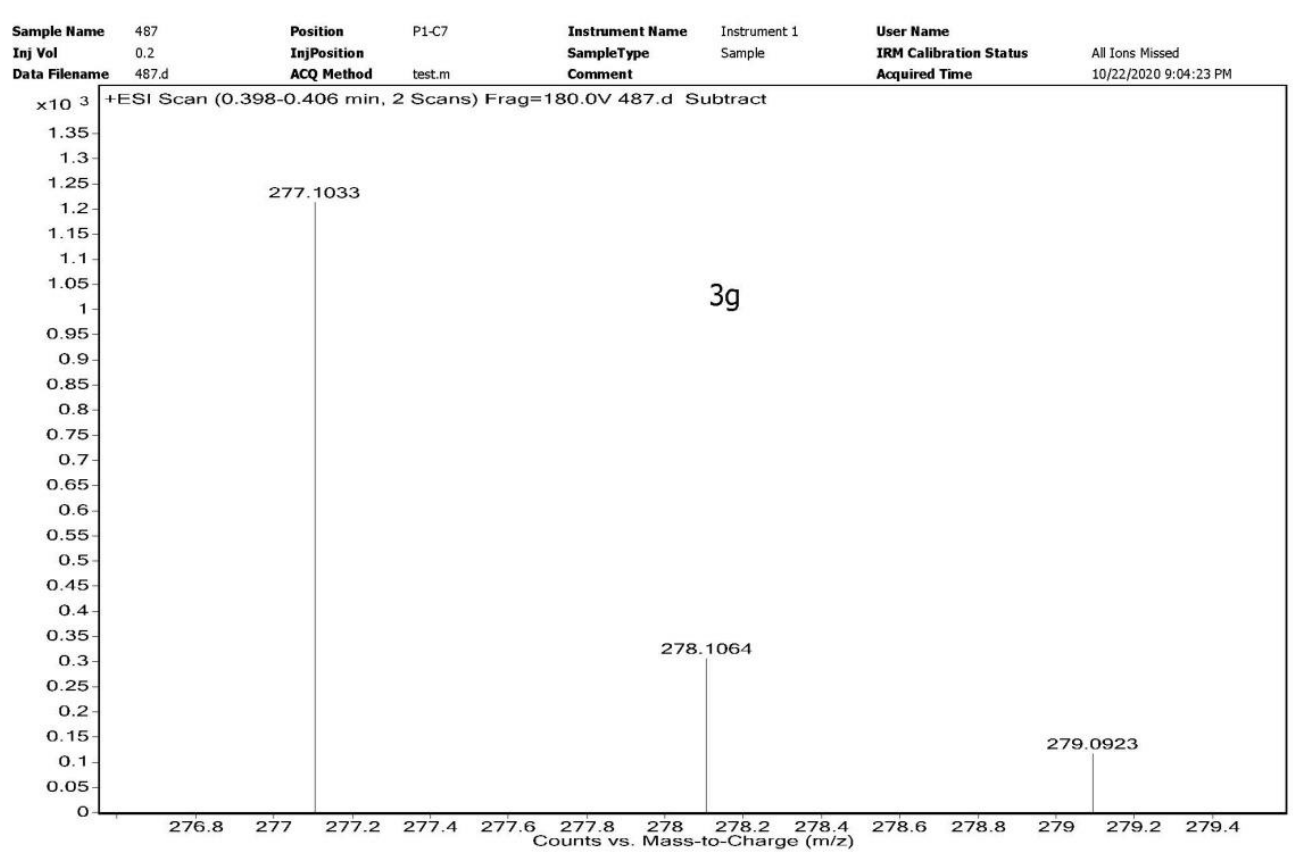


4-(3,3-difluoropropanoyl)phenyl acetate (3h): ${ }^{1} \mathrm{H}$ NMR $\left(400 \mathrm{MHz}, \mathrm{CDCl}_{3}\right)$

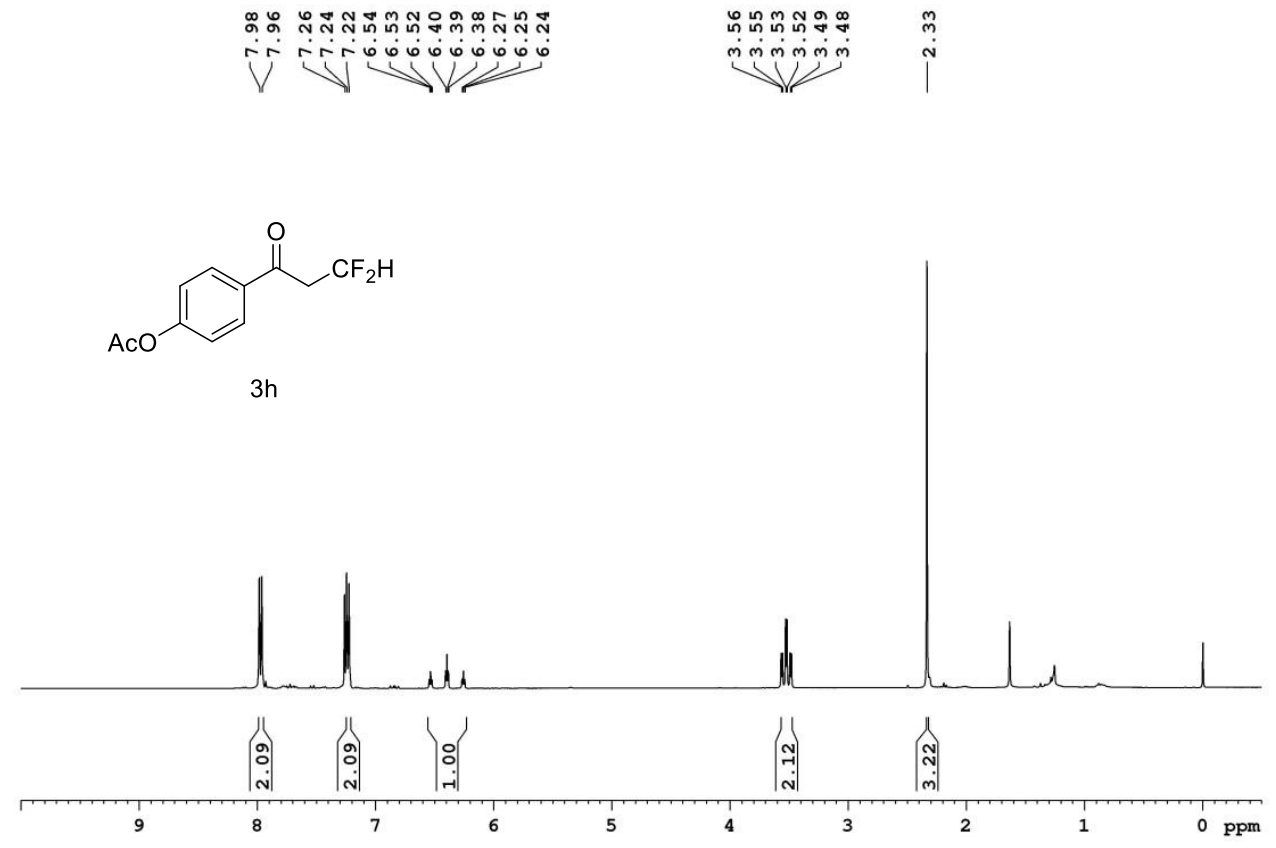

4-(3,3-difluoropropanoyl)phenyl acetate (3h): ${ }^{13} \mathrm{C}$ NMR $\left(100 \mathrm{MHz}, \mathrm{CDCl}_{3}\right)$
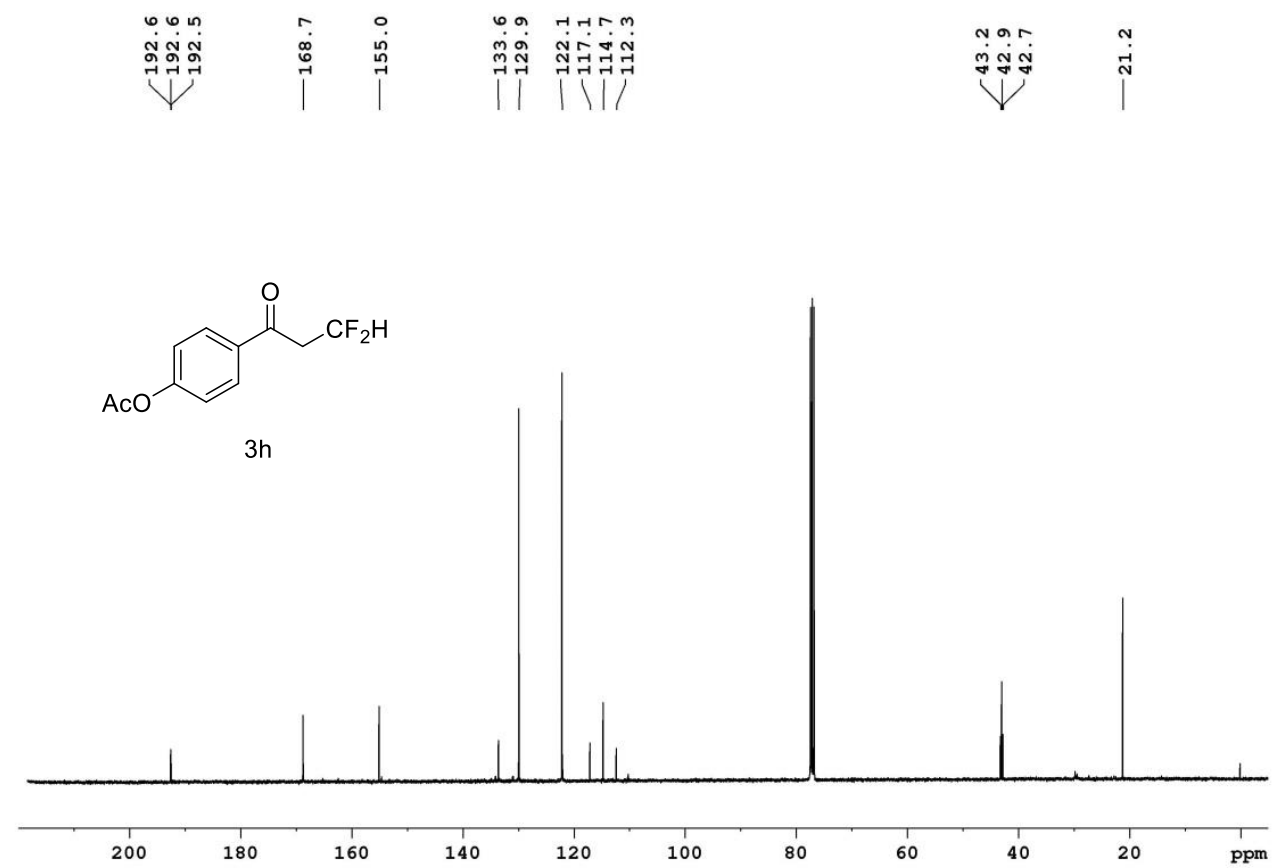
4-(3,3-difluoropropanoyl)phenyl acetate (3h): ${ }^{19} \mathrm{~F} \mathrm{NMR}\left(376.5 \mathrm{MHz}, \mathrm{CDCl}_{3}\right)$

$\infty$
0
0
1
$\mid 1$

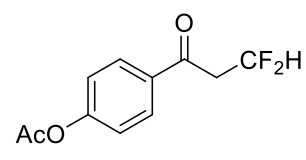

$3 \mathrm{~h}$

$-116.2$

$-116.4$

$-116.6$

$-116.8$

$-117.0$

$-117.2$

$-117.4 \mathrm{ppm}$

1-(4-(pentan-2-yl)phenyl)-3,3-difluoropropan-1-one (3i): ${ }^{1} \mathrm{H}$ NMR (400 MHz, $\left.\mathrm{CDCl}_{3}\right)$

œ

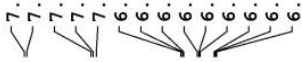

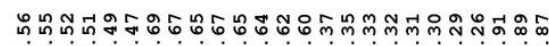

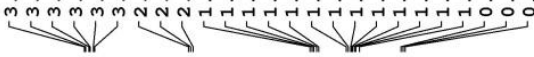

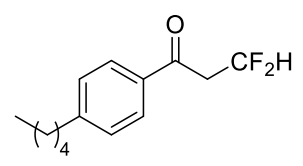

$3 i$

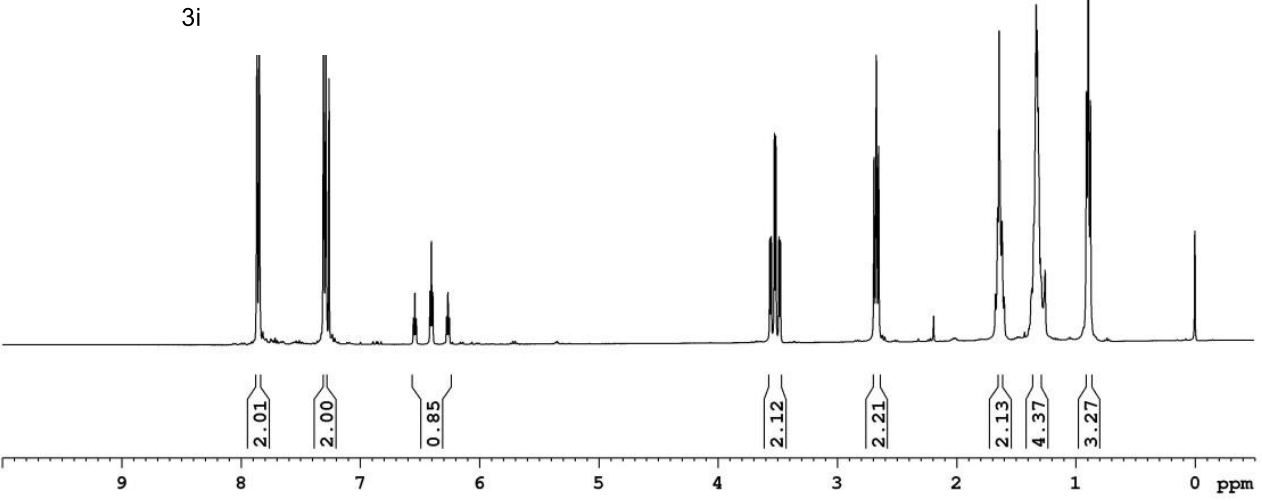


1-(4-(pentan-2-yl)phenyl)-3,3-difluoropropan-1-one (3i): $\left.{ }^{13} \mathrm{C} \mathrm{NMR} \mathrm{(100} \mathrm{MHz,} \mathrm{CDCl}_{3}\right)$

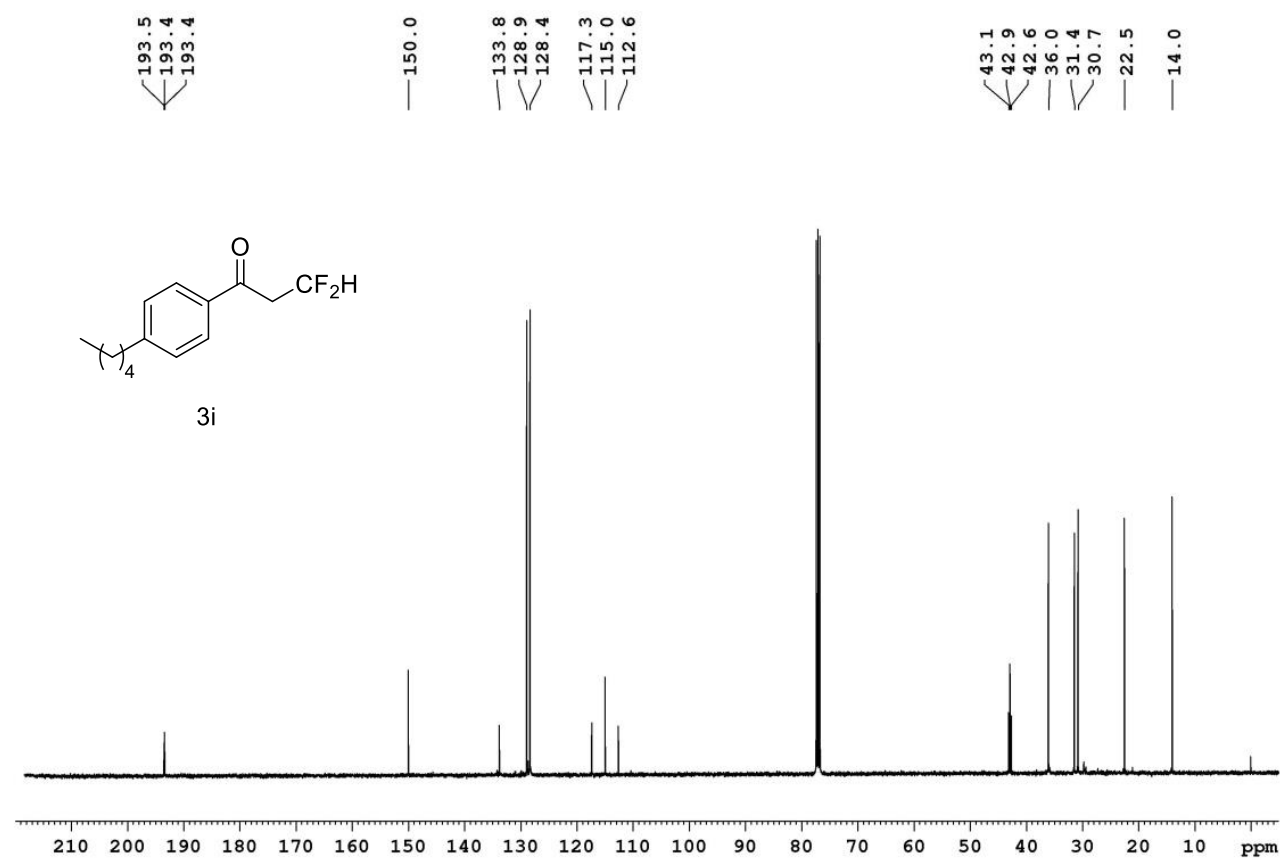

1-(4-(pentan-2-yl)phenyl)-3,3-difluoropropan-1-one (3i): ${ }^{19} \mathrm{~F}$ NMR $\left(376.5 \mathrm{MHz}, \mathrm{CDCl}_{3}\right)$

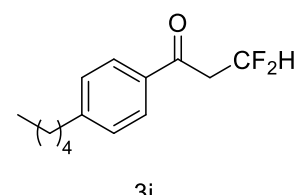

$3 \mathbf{i}$ 
1-(4-(pentan-2-yl)phenyl)-3,3-difluoropropan-1-one (3i): (HRMS)

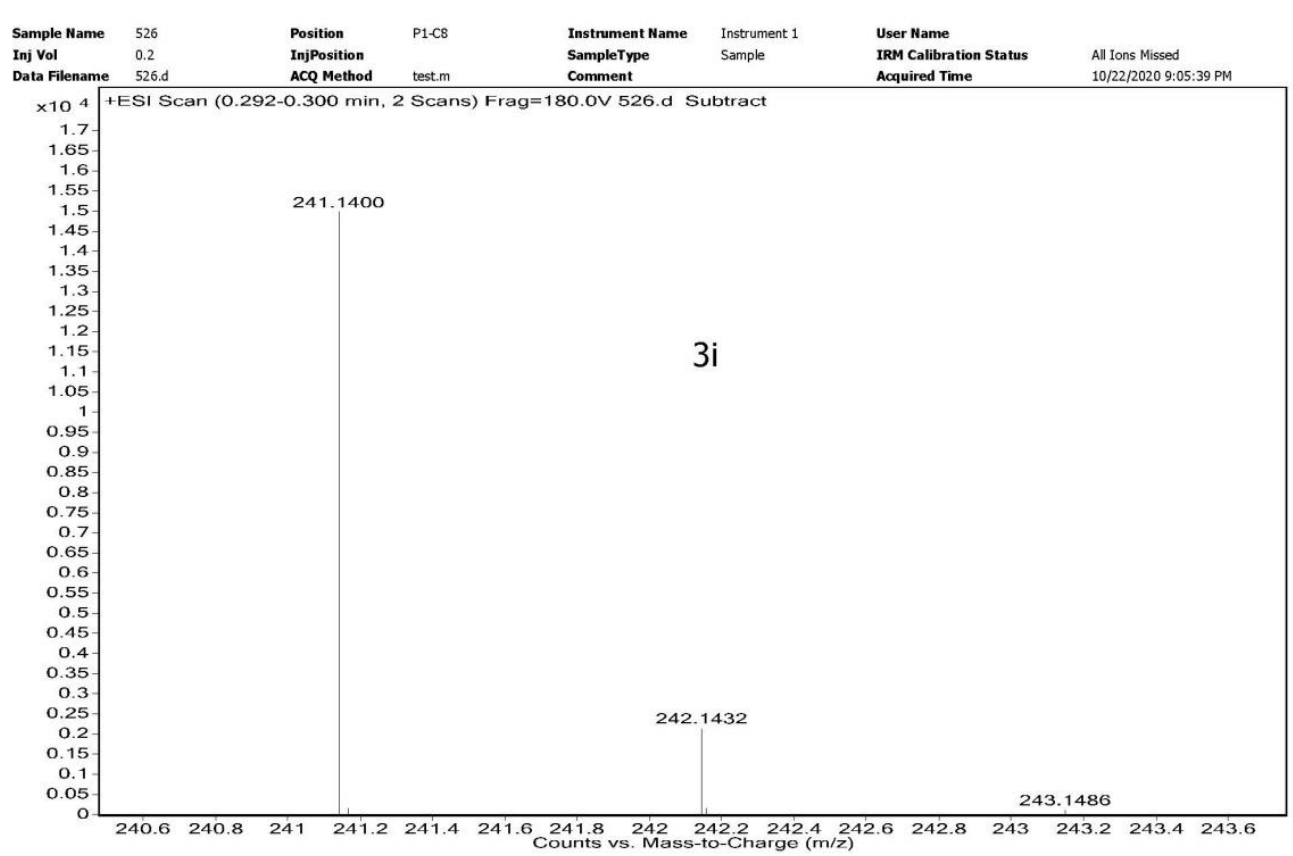

3,3-difluoro-1-(m-tolyl)propan-1-one (3j): ${ }^{1} \mathrm{H}$ NMR (400 MHz, $\mathrm{CDCl}_{3}$ )

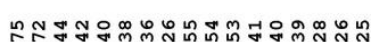

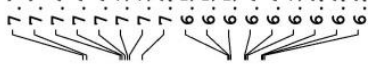<smiles>Cc1cccc(C(=O)CC(F)F)c1</smiles>

3j

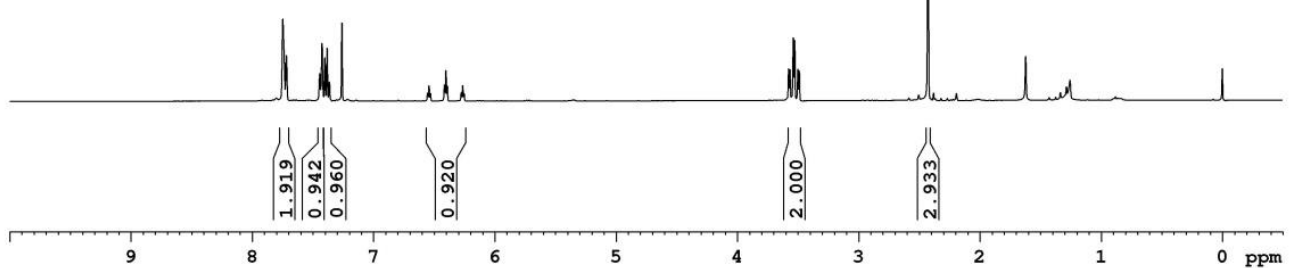


3,3-difluoro-1-(m-tolyl)propan-1-one (3j): ${ }^{13} \mathrm{C} \mathrm{NMR} \mathrm{(100} \mathrm{MHz,} \mathrm{CDCl}_{3}$ )

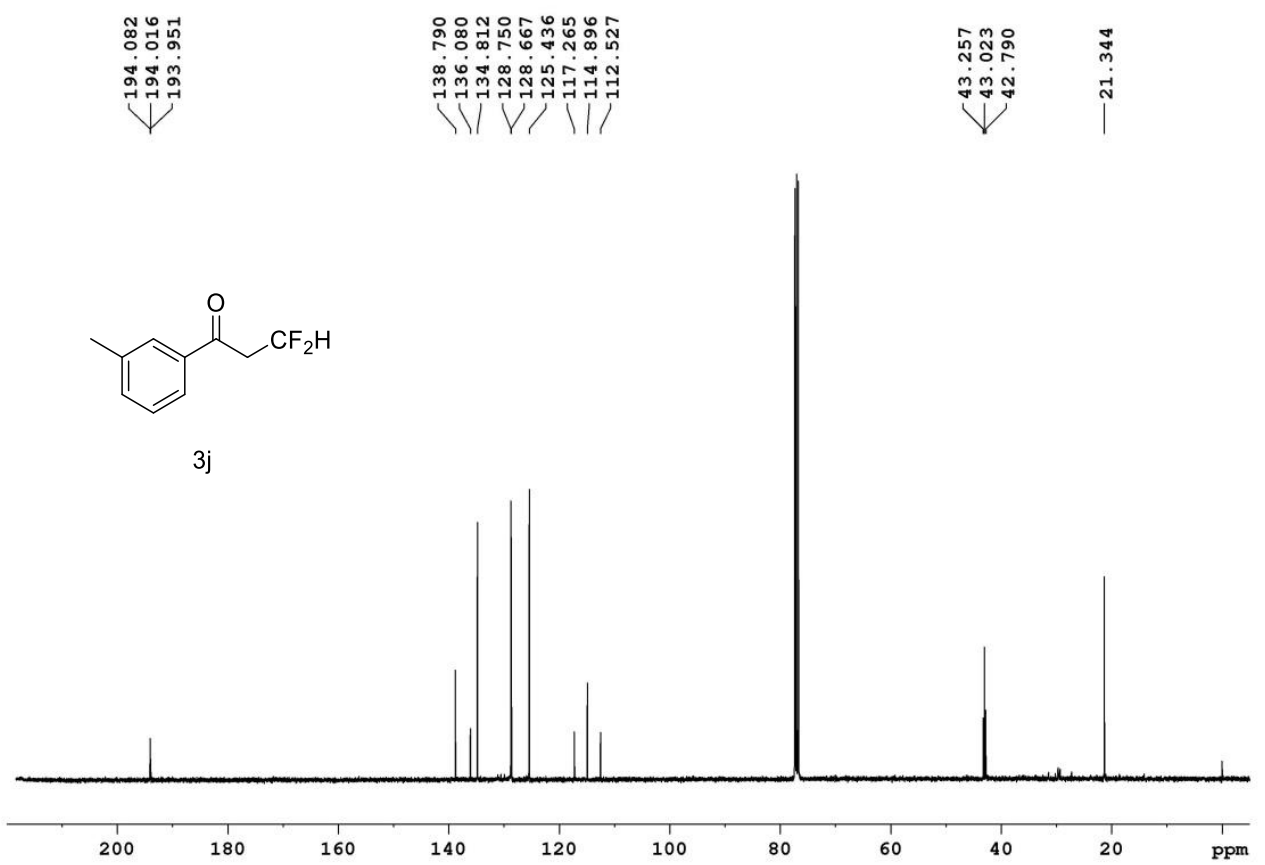

3,3-difluoro-1-(m-tolyl)propan-1-one (3j): ${ }^{19} \mathrm{~F}$ NMR (376.5 MHz, $\left.\mathrm{CDCl}_{3}\right)$

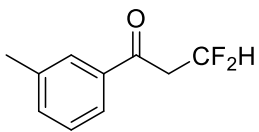

9
0
7
1

3j

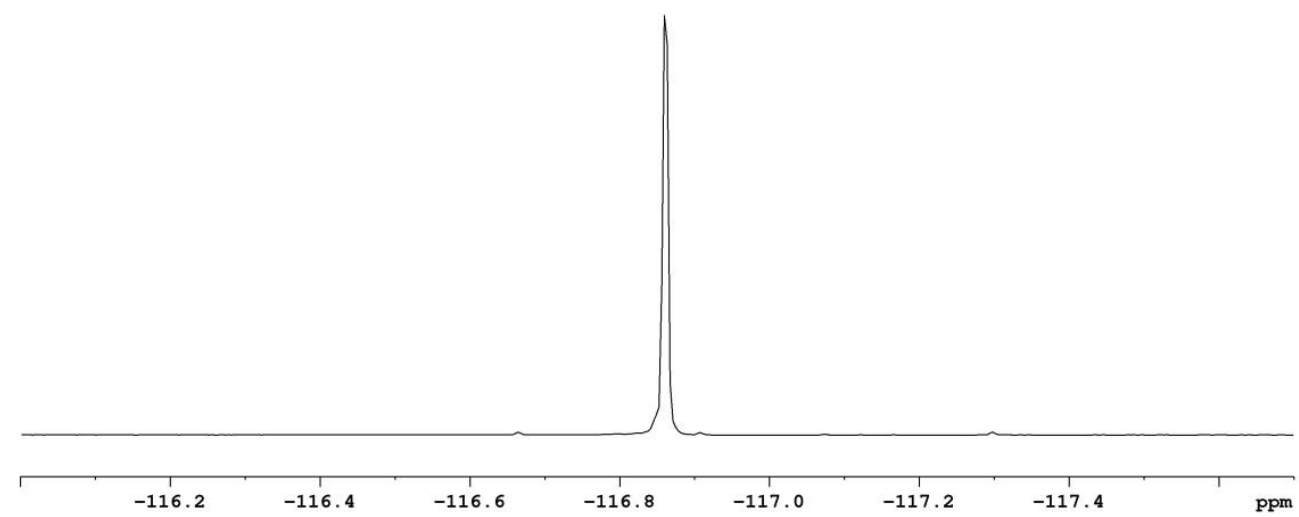


1-(4-(tert-butyl)phenyl)-3,3-difluoropropan-1-one (3k): ${ }^{1} \mathrm{H} \mathrm{NMR}\left(400 \mathrm{MHz}, \mathrm{CDCl}_{3}\right)$

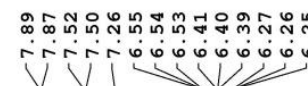

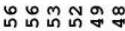

mimmim

$\stackrel{\stackrel{n}{m}}{i}$<smiles>CC(C)Cc1ccc(C(=O)CC(F)F)cc1</smiles>

$3 k$

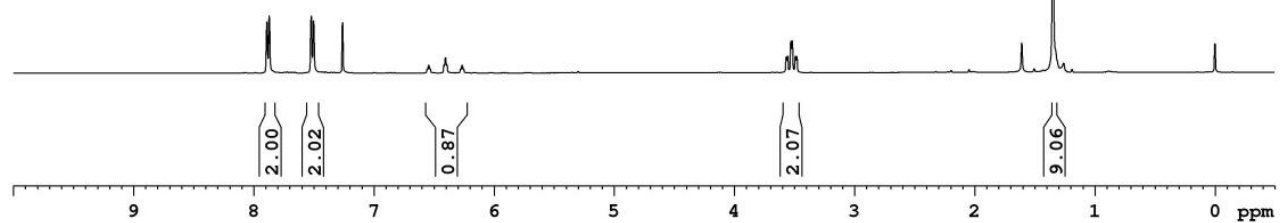

1-(4-(tert-butyl)phenyl)-3,3-difluoropropan-1-one (3k): ${ }^{13} \mathrm{C} \mathrm{NMR}\left(100 \mathrm{MHz}, \mathrm{CDCl}_{3}\right)$

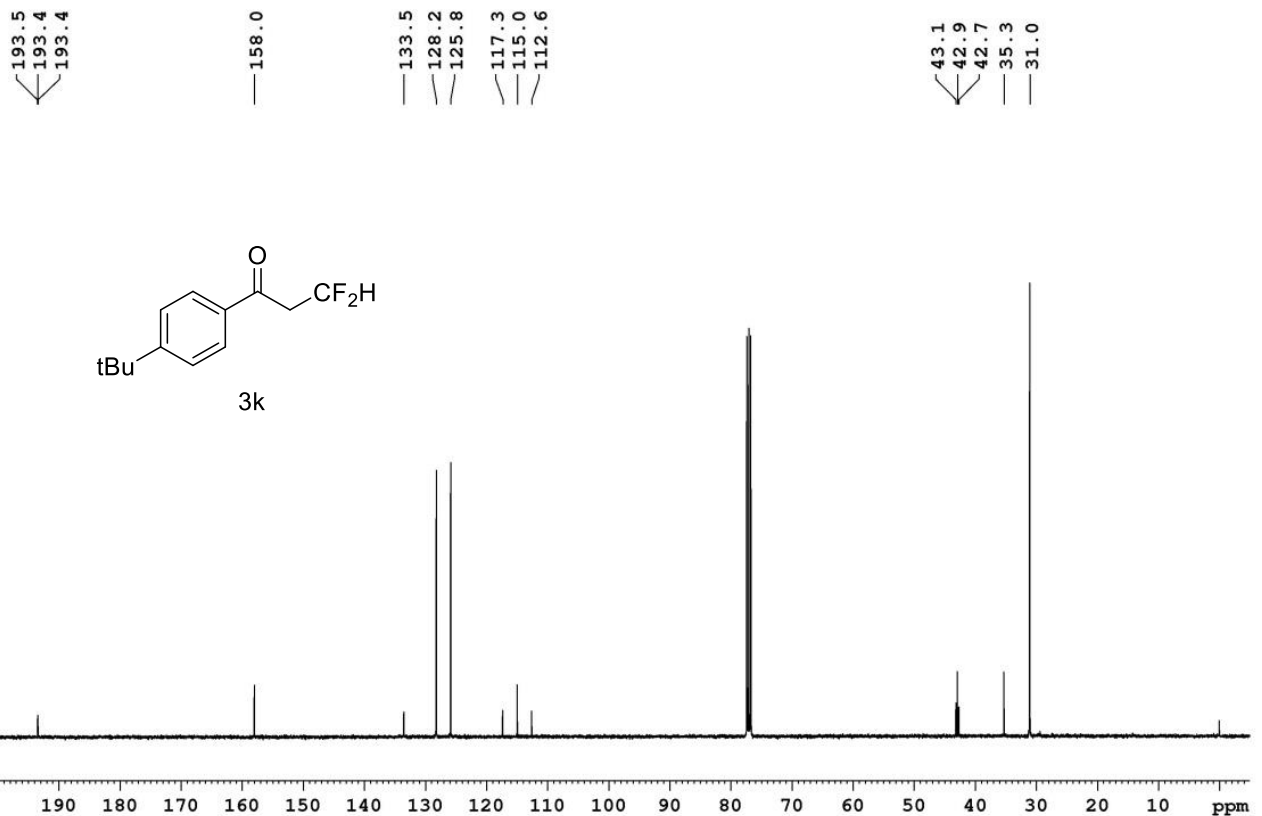


1-(4-(tert-butyl)phenyl)-3,3-difluoropropan-1-one (3k): ${ }^{19} \mathrm{~F}$ NMR (376.5 MHz, $\mathrm{CDCl}_{3}$ )

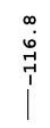<smiles>CC(C)(C)c1ccc(C(=O)CC(F)(F)F)cc1</smiles>

$3 \mathrm{k}$

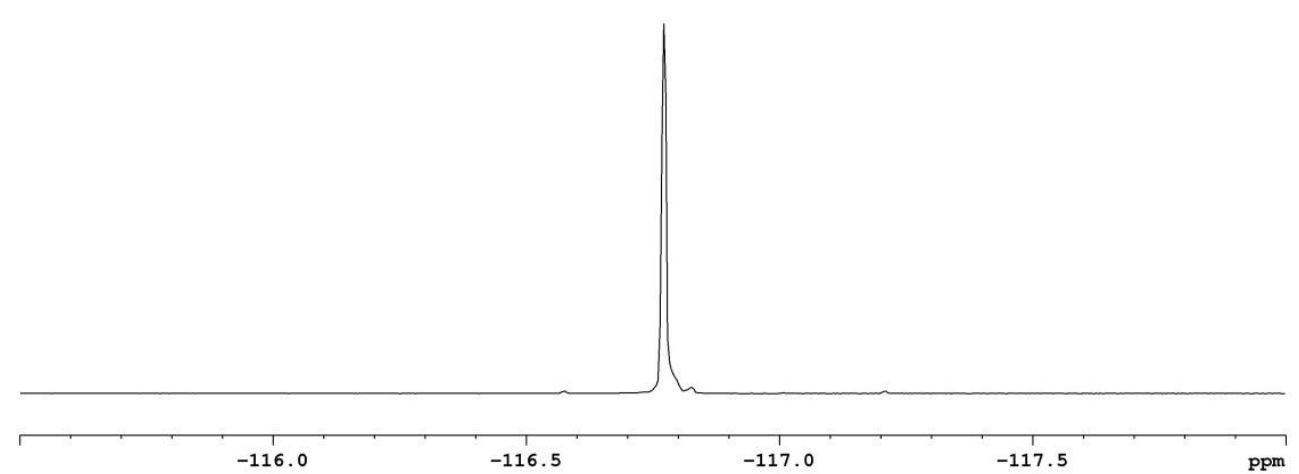

3,3-difluoro-1-(4-phenoxyphenyl)propan-1-one (3I): ${ }^{1} \mathrm{H} \mathrm{NMR}\left(400 \mathrm{MHz}, \mathrm{CDCl}_{3}\right)$

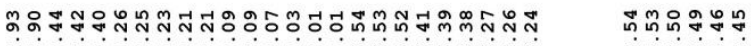

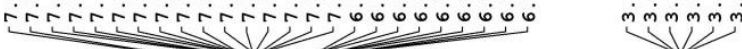

$\mathrm{CF}_{2} \mathrm{H}$

31

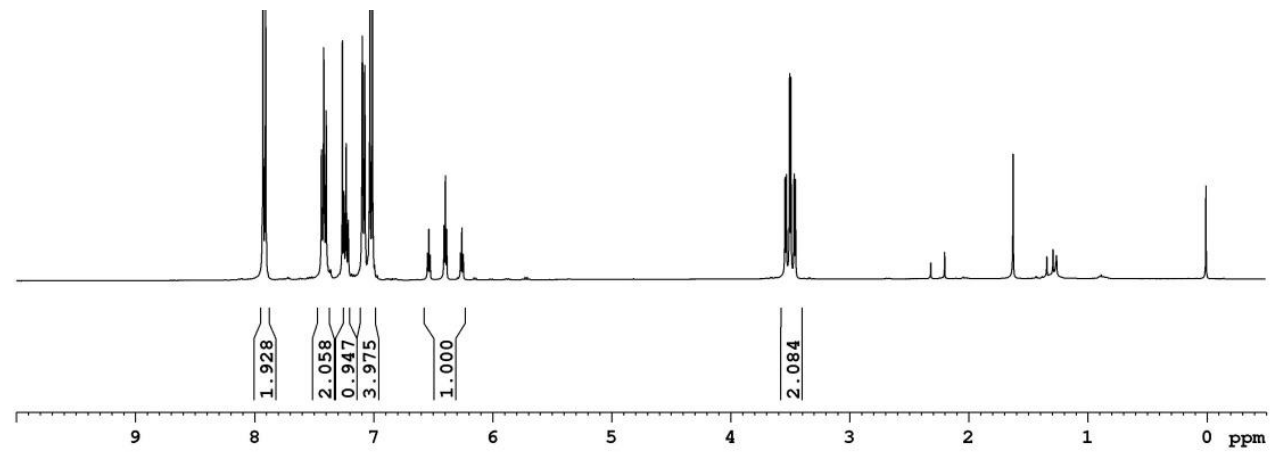


3,3-difluoro-1-(4-phenoxyphenyl)propan-1-one (31): $\left.{ }^{13} \mathrm{C} \mathrm{NMR} \mathrm{(100} \mathrm{MHz}, \mathrm{CDCl}_{3}\right)$

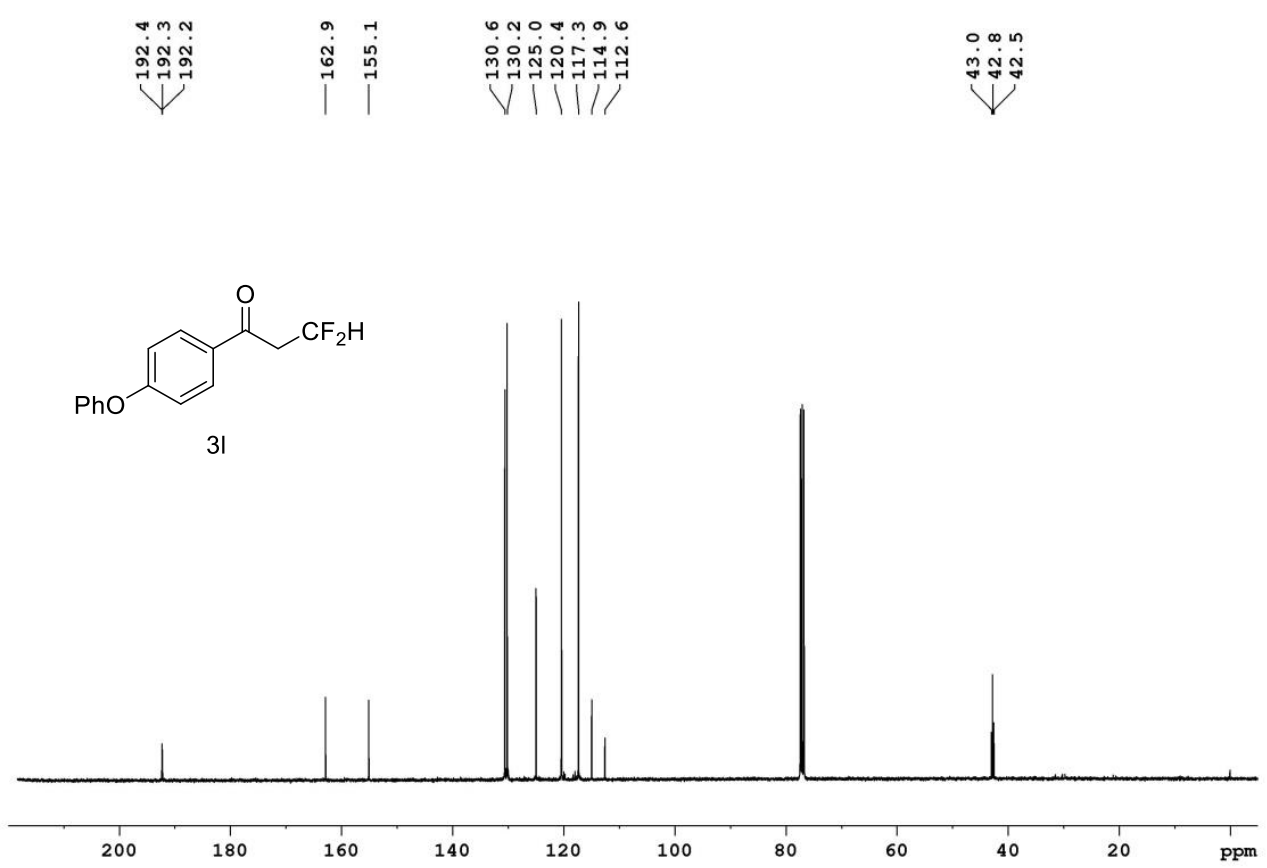

3,3-difluoro-1-(4-phenoxyphenyl)propan-1-one (3I): ${ }^{19} \mathrm{~F}$ NMR (376.5 MHz, $\left.\mathrm{CDCl}_{3}\right)$

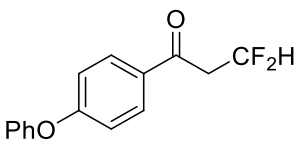

$\overrightarrow{0}$

31

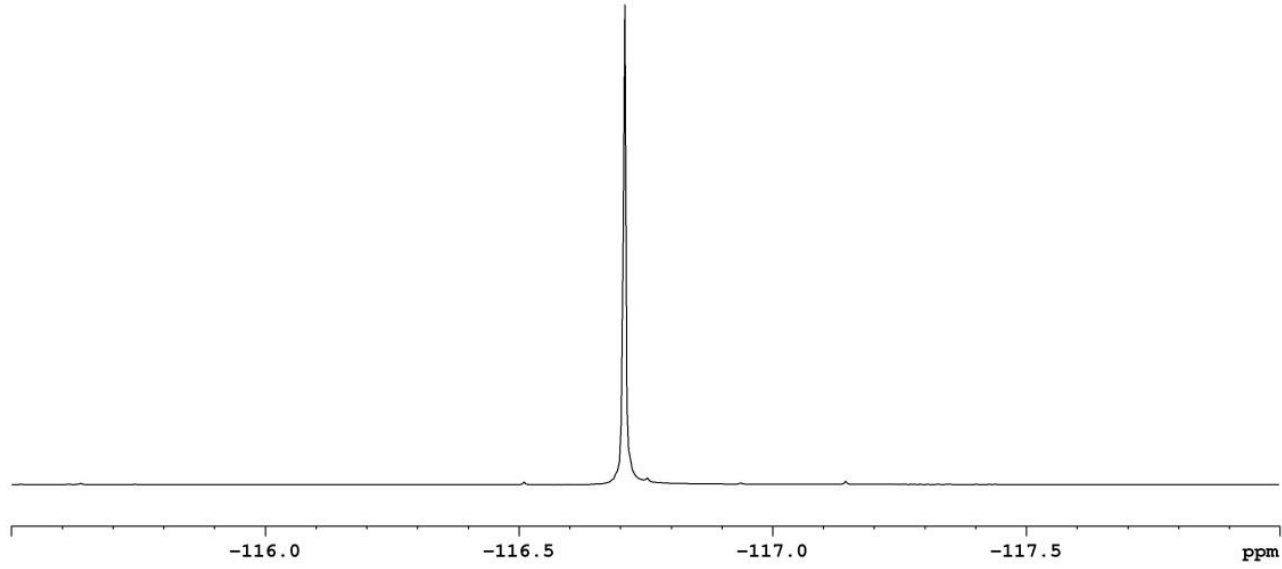


1-(3,4-dimethoxyphenyl)-3,3-difluoropropan-1-one (3m): ${ }^{1} \mathrm{H} \mathrm{NMR}\left(400 \mathrm{MHz}, \mathrm{CDCl}_{3}\right)$

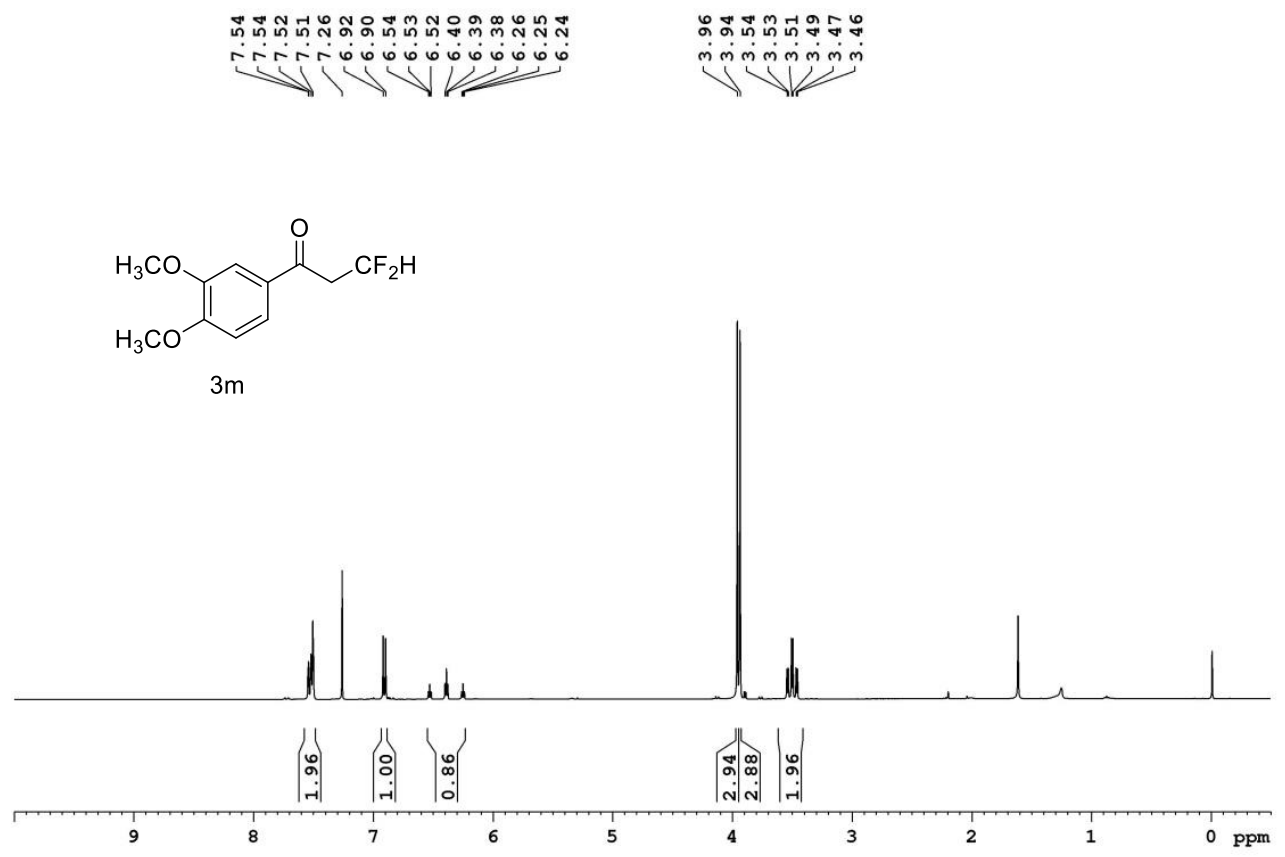

1-(3,4-dimethoxyphenyl)-3,3-difluoropropan-1-one (3m): $\left.{ }^{13} \mathrm{C} \mathrm{NMR} \mathrm{(100} \mathrm{MHz,} \mathrm{CDCl}_{3}\right)$

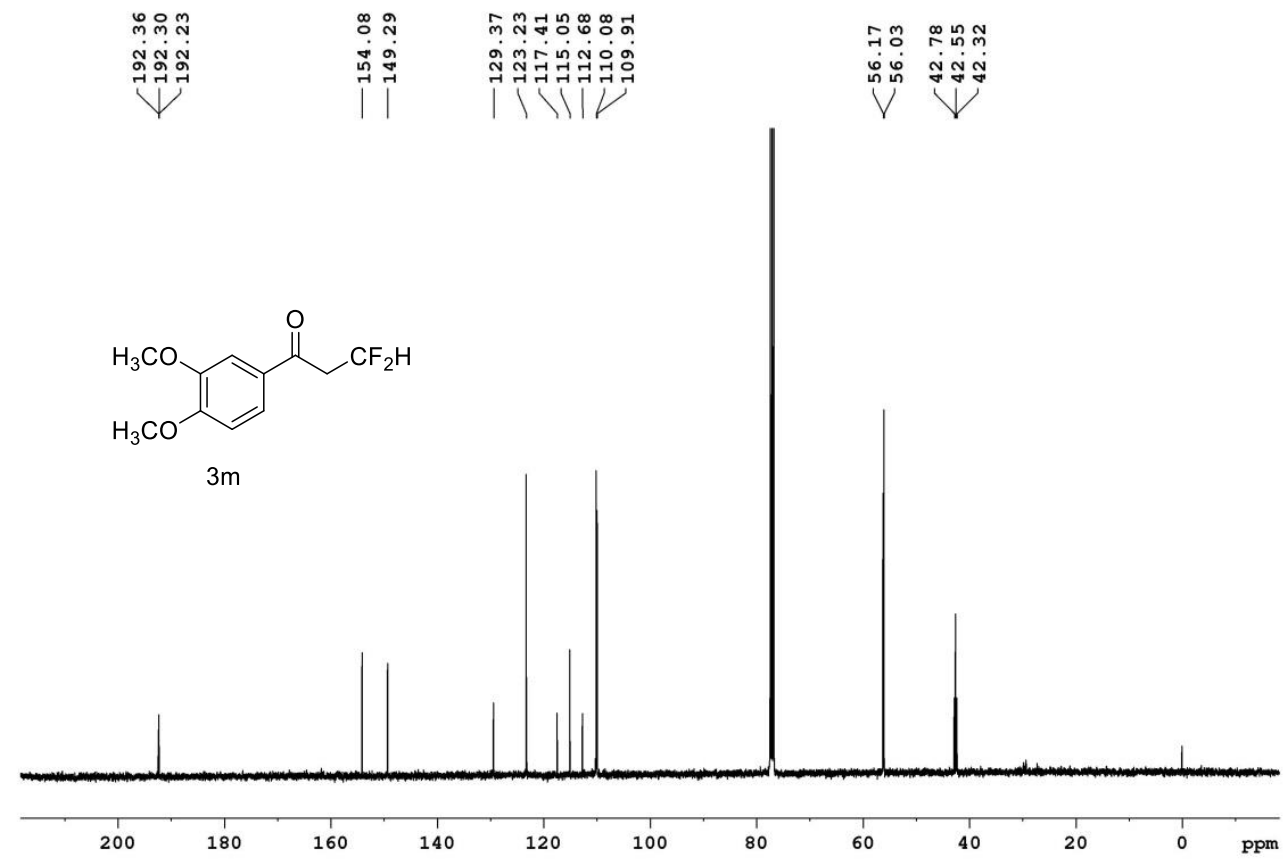


1-(3,4-dimethoxyphenyl)-3,3-difluoropropan-1-one (3m): ${ }^{19} \mathrm{~F}$ NMR (376.5 MHz, $\left.\mathrm{CDCl}_{3}\right)$

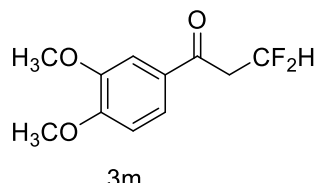

$3 m$

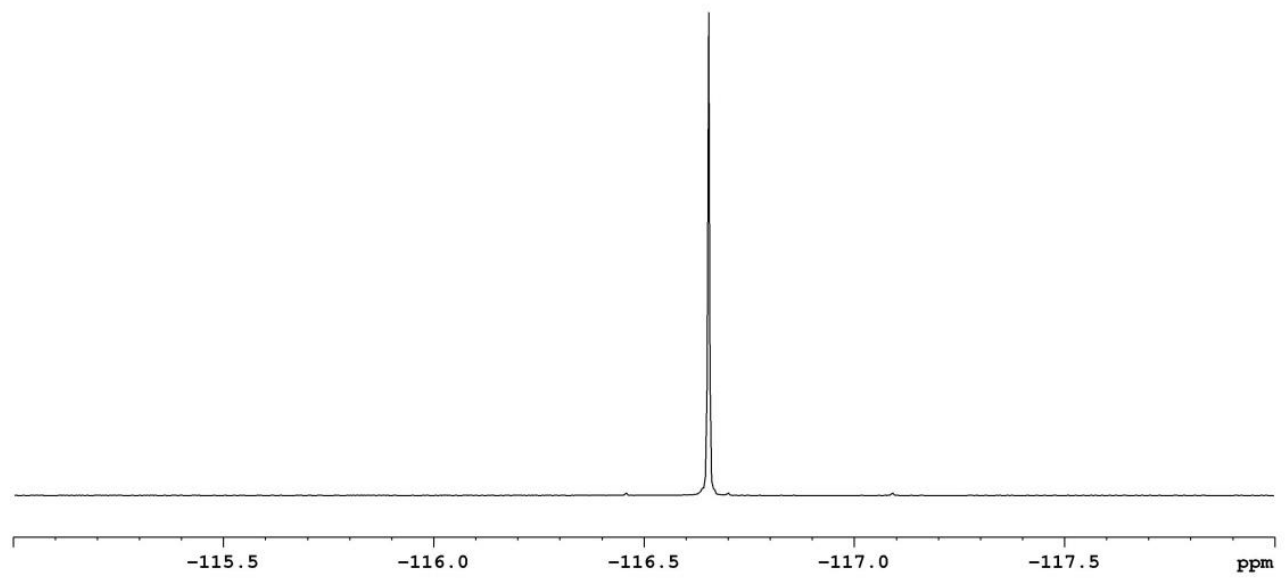

1-(3,4-dimethoxyphenyl)-3,3-difluoropropan-1-one (3m): (HRMS)

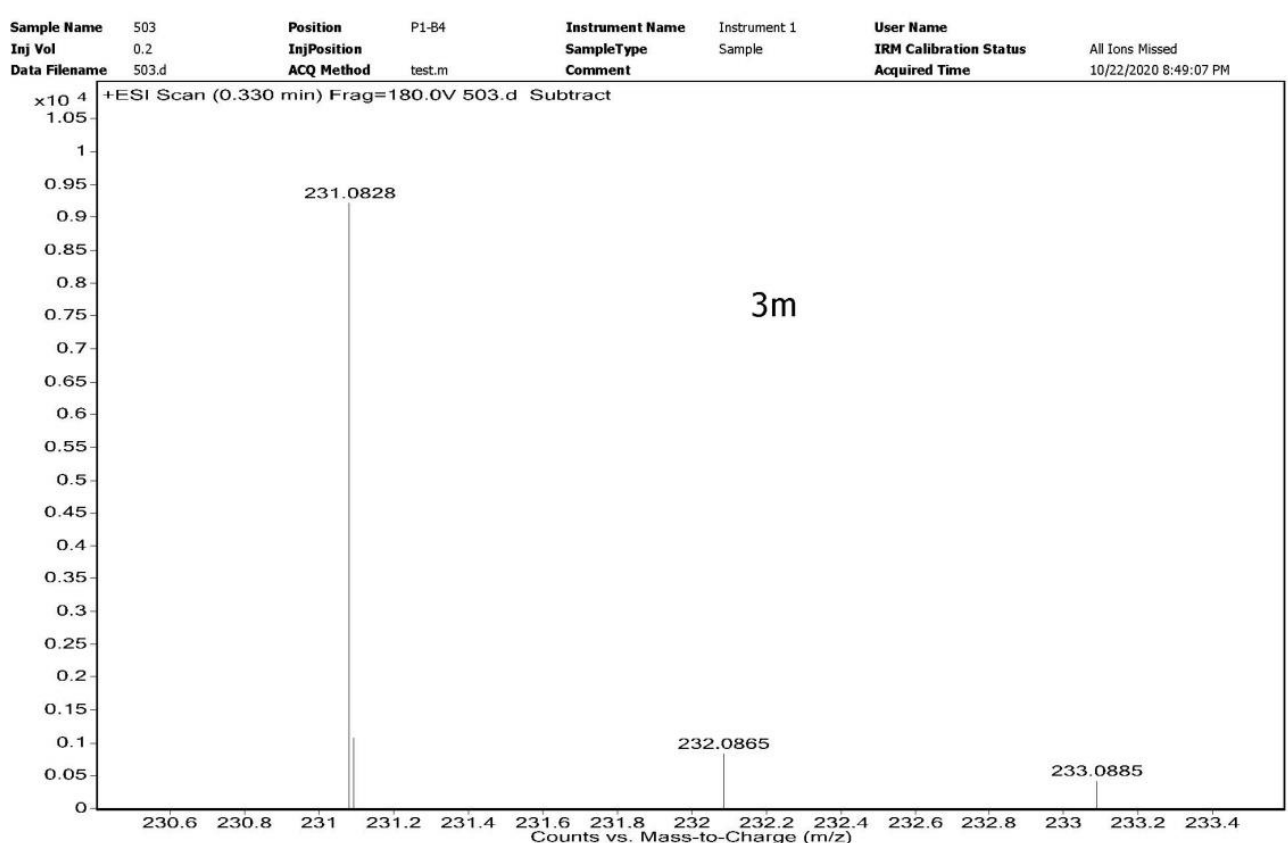


3,3-difluoro-1-(2-fluoro-4-methoxyphenyl)propan-1-one (3n): ${ }^{1} \mathrm{H} \mathrm{NMR}\left(400 \mathrm{MHz}, \mathrm{CDCl}_{3}\right)$

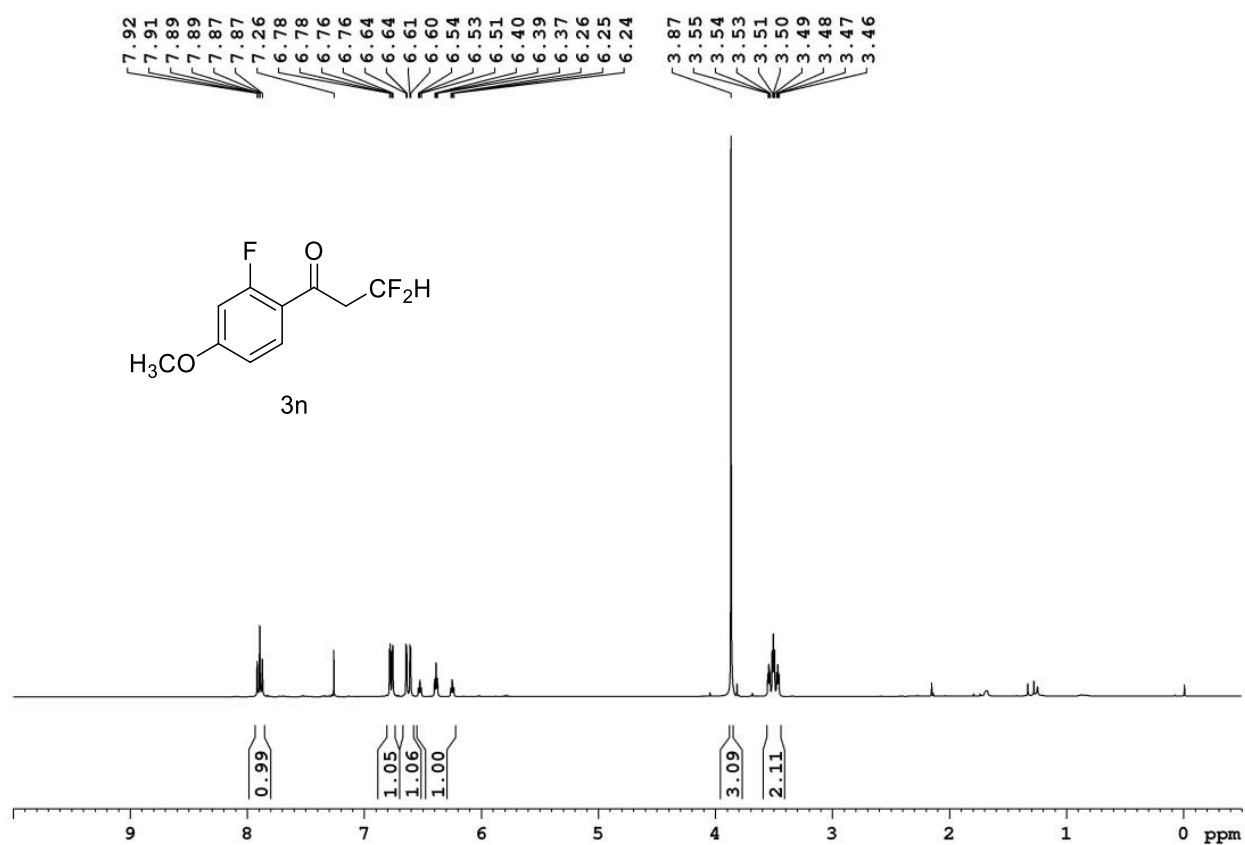

3,3-difluoro-1-(2-fluoro-4-methoxyphenyl)propan-1-one (3n): ${ }^{13} \mathrm{C} \mathrm{NMR}\left(100 \mathrm{MHz}, \mathrm{CDCl}_{3}\right)$
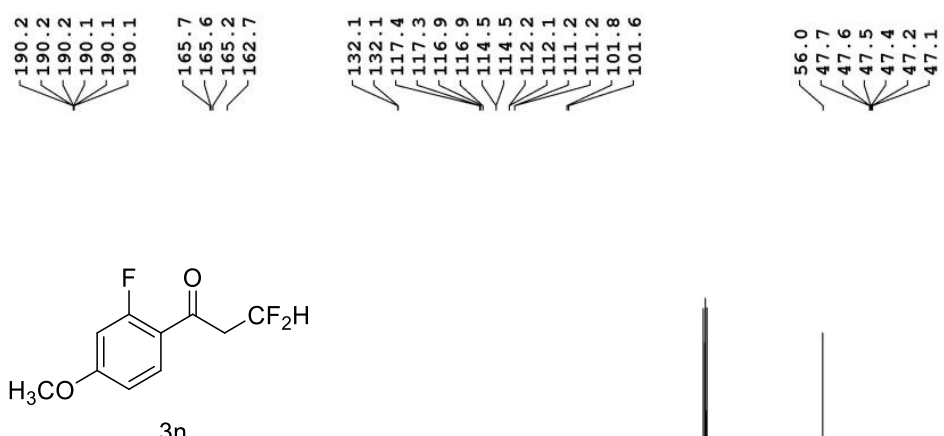

$3 n$

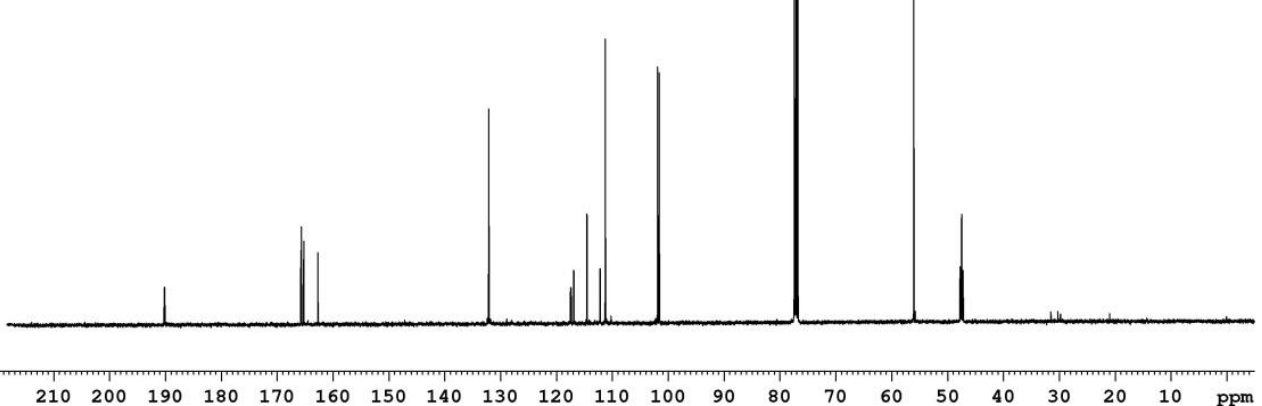


3,3-difluoro-1-(2-fluoro-4-methoxyphenyl)propan-1-one (3n): ${ }^{19} \mathrm{~F}$ NMR (376.5 MHz, $\left.\mathrm{CDCl}_{3}\right)$

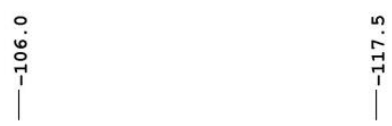

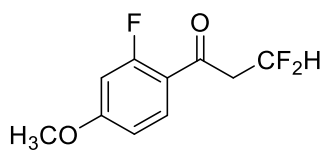

$3 n$

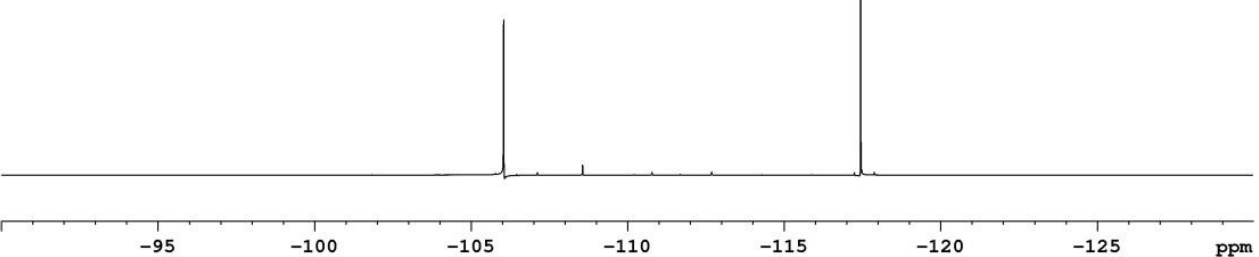

3,3-difluoro-1-(2-fluoro-4-methoxyphenyl)propan-1-one (3n): (HRMS)

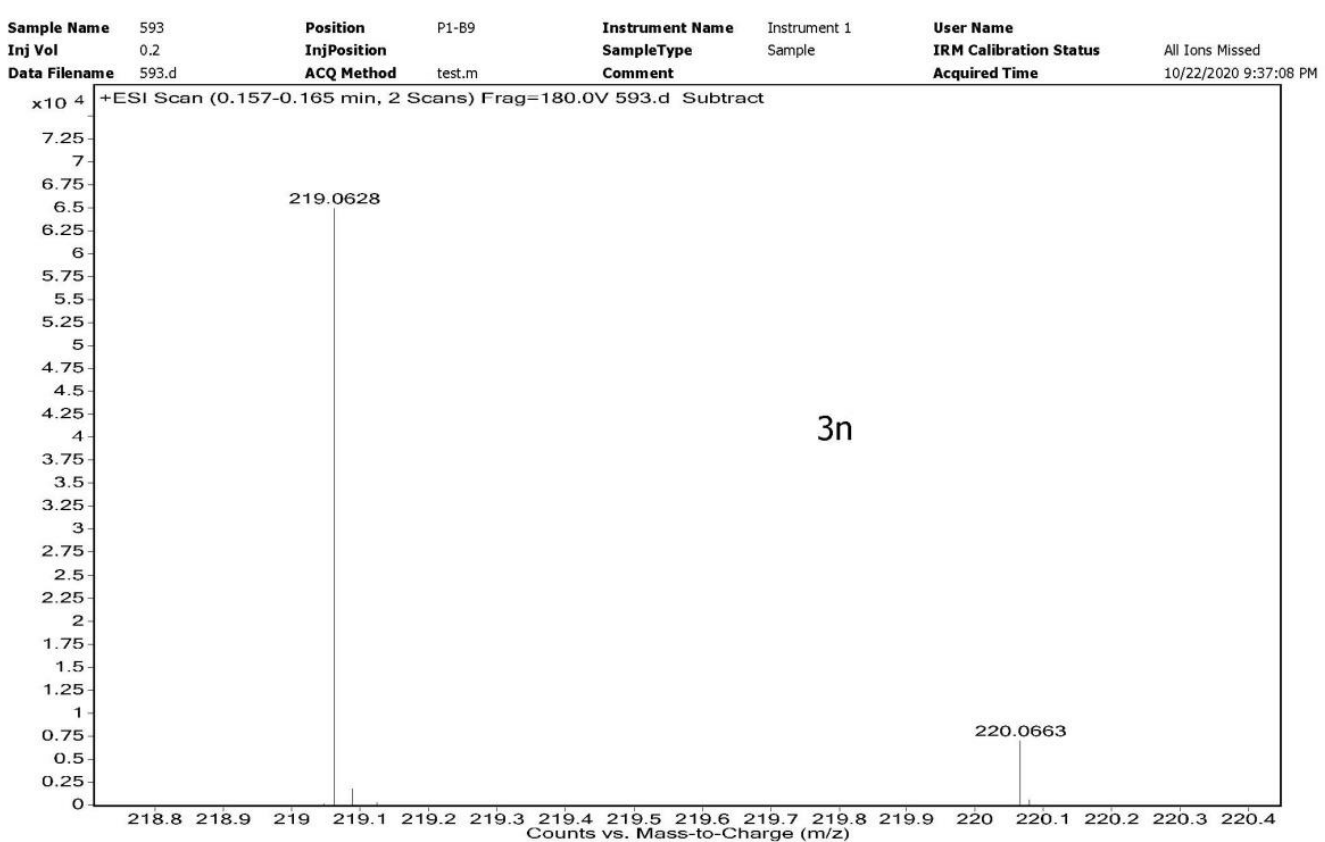


1-(3,4-dimethylphenyl)-3,3-difluoropropan-1-one (3o): ${ }^{1} \mathrm{HNMR}\left(400 \mathrm{MHz}, \mathrm{CDCl}_{3}\right)$

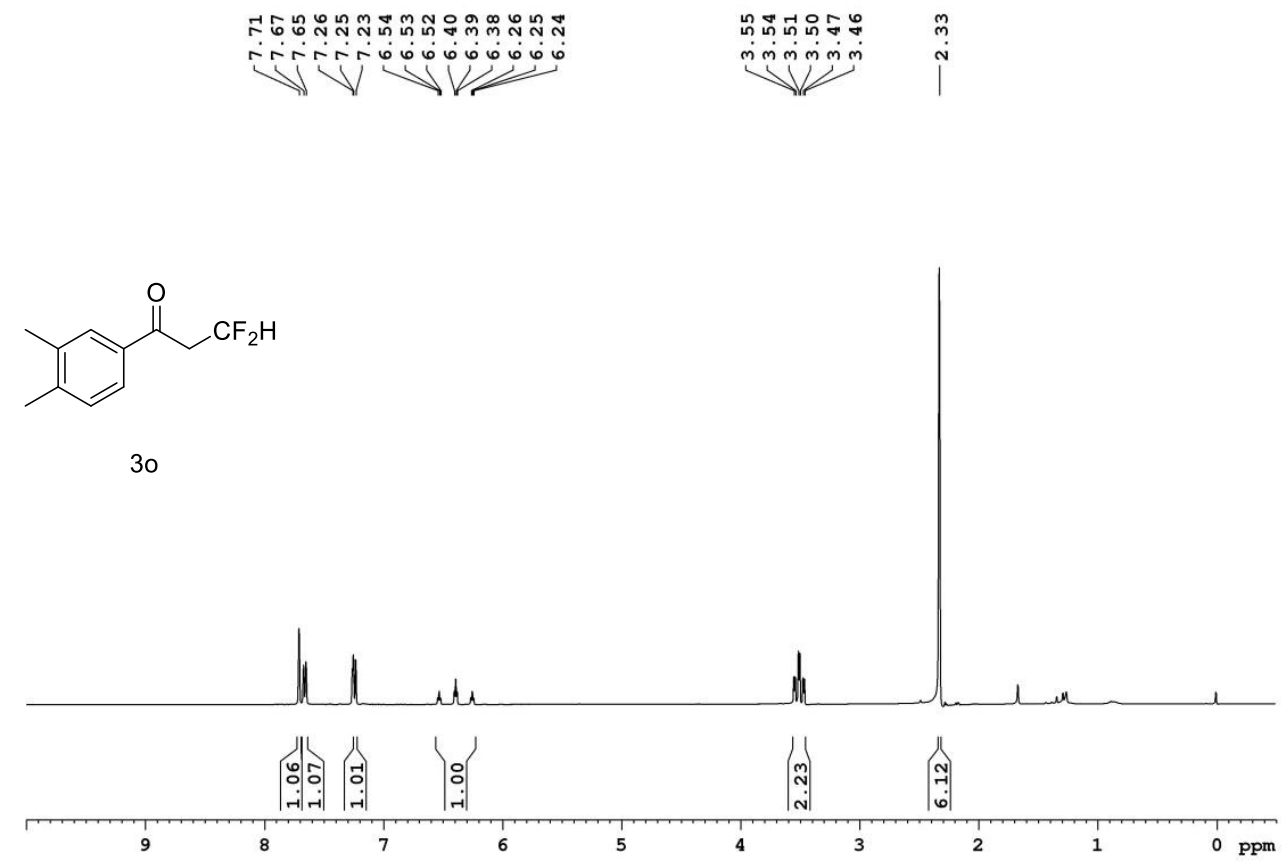

1-(3,4-dimethylphenyl)-3,3-difluoropropan-1-one (3o): ${ }^{13} \mathrm{C} \mathrm{NMR}\left(100 \mathrm{MHz}, \mathrm{CDCl}_{3}\right)$

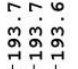

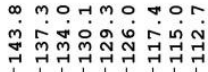

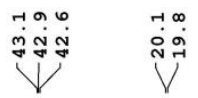

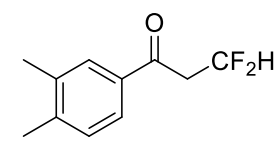

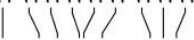

30

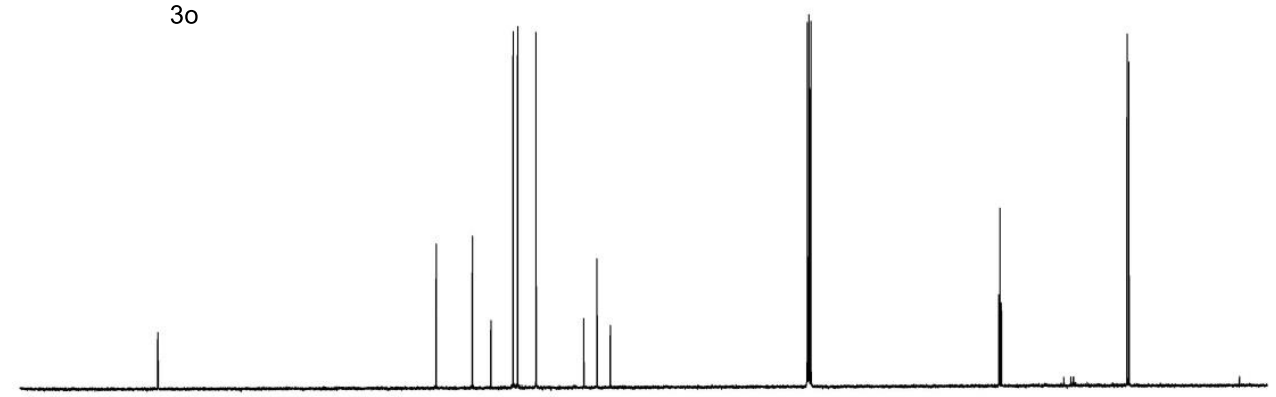

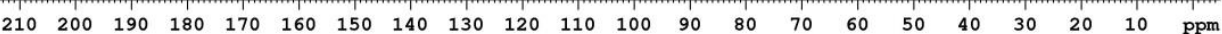


1-(3,4-dimethylphenyl)-3,3-difluoropropan-1-one (3o): ${ }^{19} \mathrm{~F} \mathrm{NMR}\left(376.5 \mathrm{MHz}, \mathrm{CDCl}_{3}\right)$

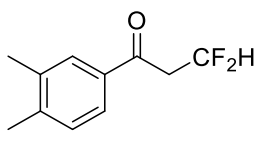

$\infty$
0
-1
7

30

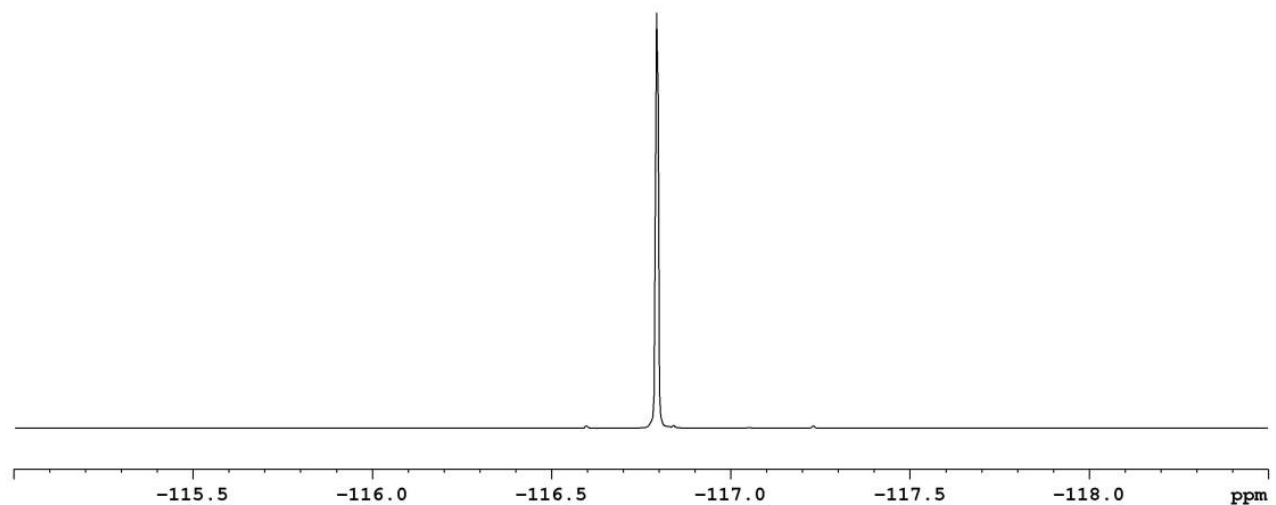

1-(3,4-dimethylphenyl)-3,3-difluoropropan-1-one (3o): (HRMS)

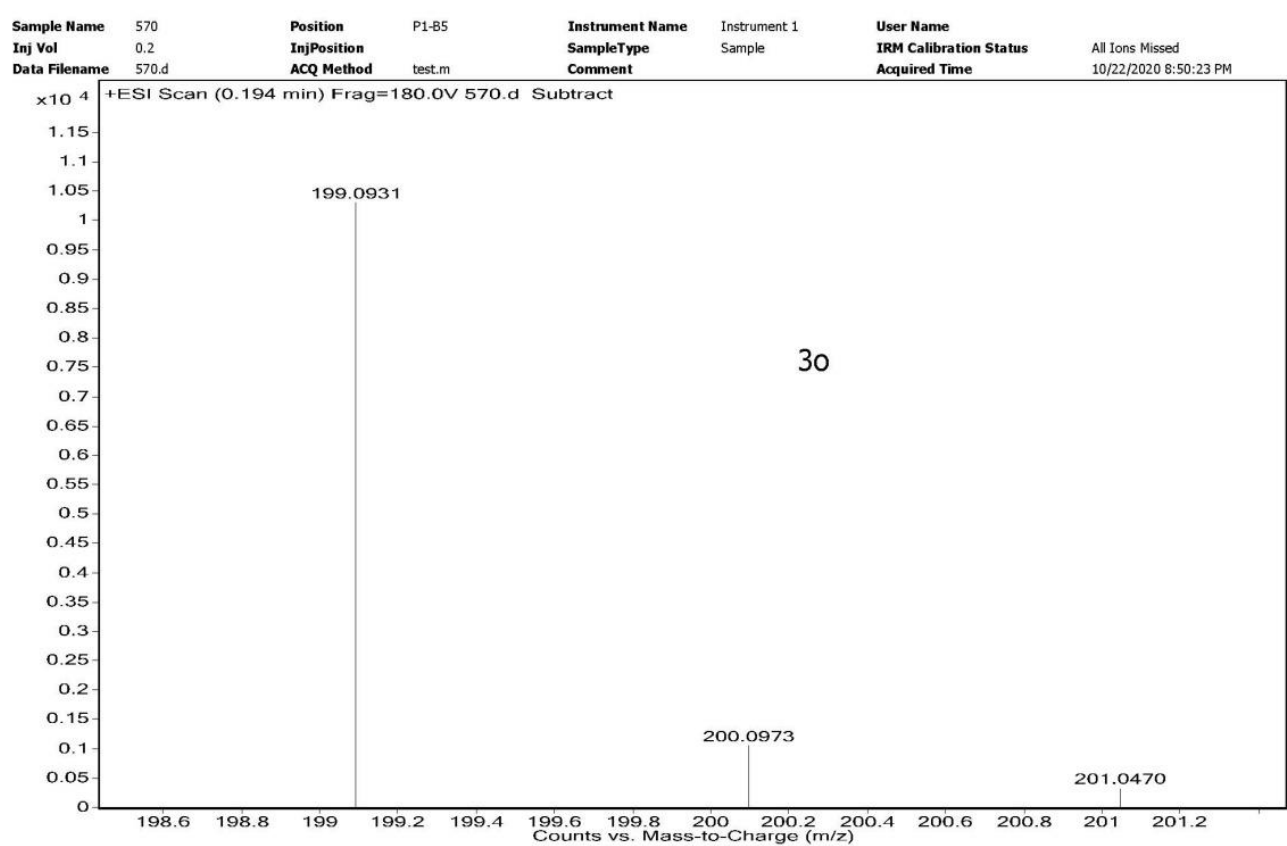


1-(9H-fluoren-2-yl)-3,3-difluoropropan-1-one (3p): ${ }^{1} \mathrm{H}$ NMR (400 MHz, $\left.\mathrm{CDCl}_{3}\right)$

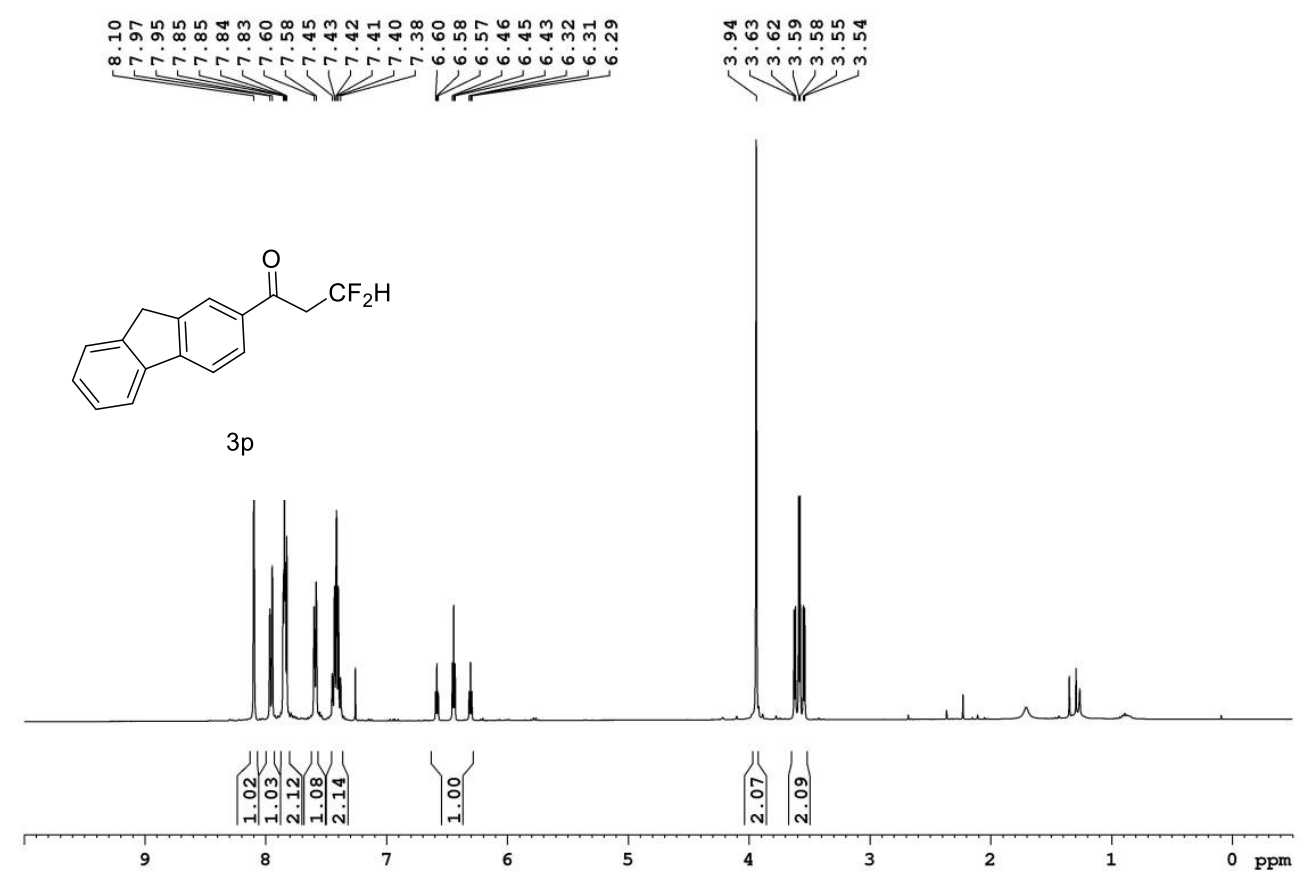

1-(9H-fluoren-2-yl)-3,3-difluoropropan-1-one (3p): $\left.{ }^{13} \mathrm{C} \mathrm{NMR} \mathrm{(100} \mathrm{MHz,} \mathrm{CDCl}_{3}\right)$
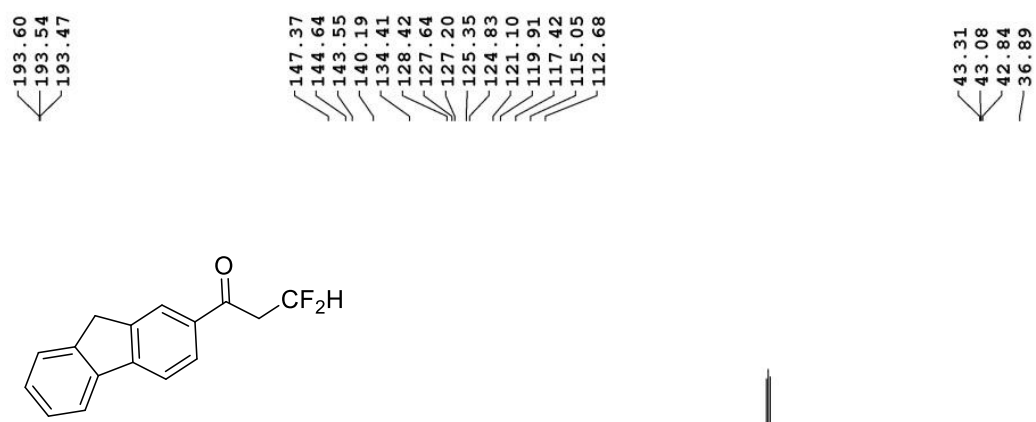

$3 p$

i

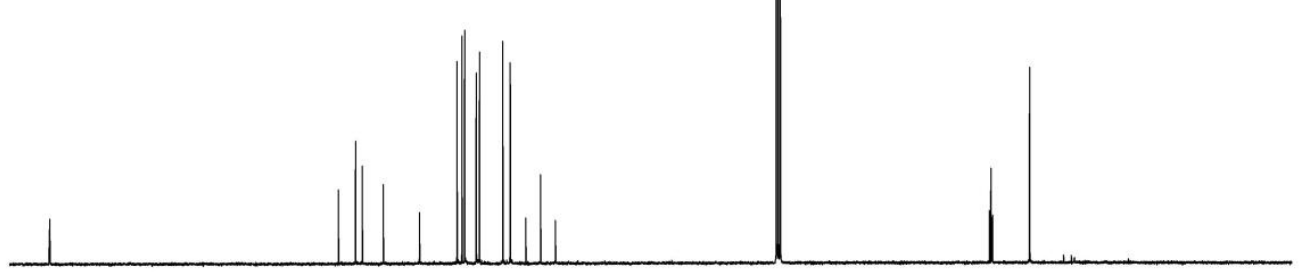

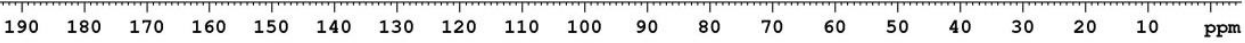


1-(9H-fluoren-2-yl)-3,3-difluoropropan-1-one (3p): $\left.{ }^{19} \mathrm{~F} \mathrm{NMR} \mathrm{(376.5} \mathrm{MHz}, \mathrm{CDCl}_{3}\right)$

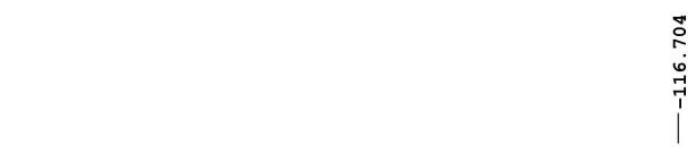

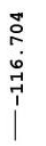

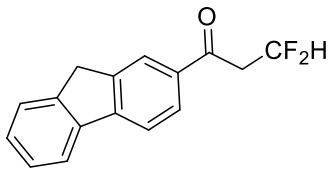

$3 p$

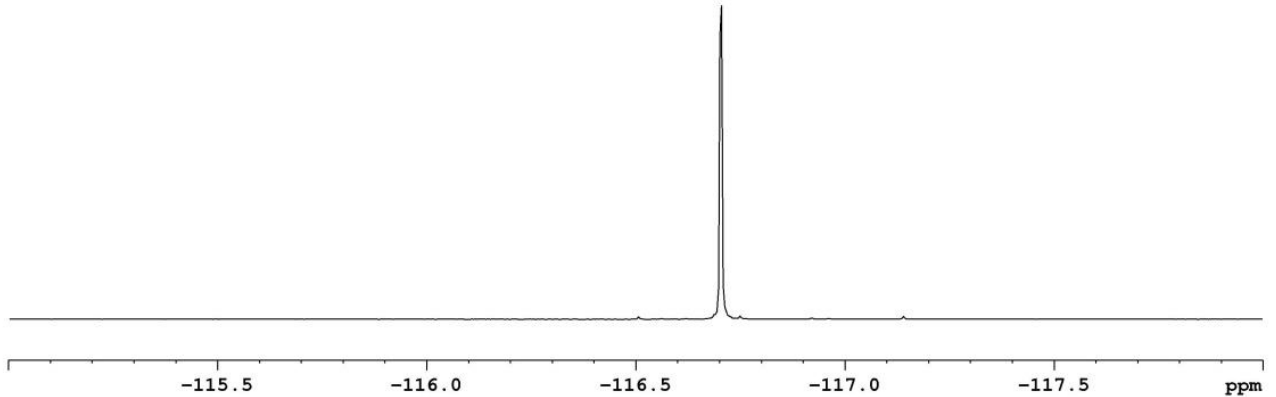

3,3-difluoro-1-(4-fluorophenyl)propan-1-one (3q): ${ }^{1} \mathrm{H}$ NMR (400 MHz, $\left.\mathrm{CDCl}_{3}\right)$

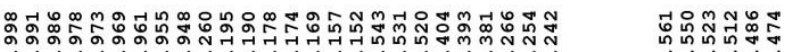

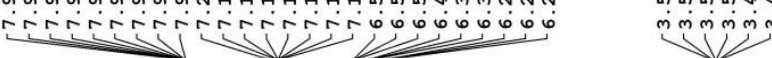

$\mathrm{CF}_{2} \mathrm{H}$

$3 q$

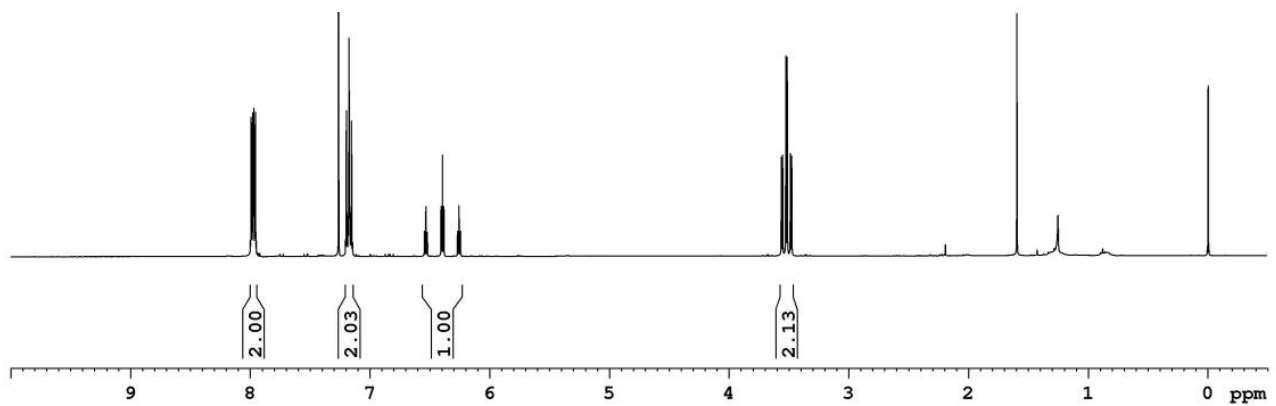


3,3-difluoro-1-(4-fluorophenyl)propan-1-one (3q): ${ }^{13} \mathrm{C} \mathrm{NMR}\left(100 \mathrm{MHz}, \mathrm{CDCl}_{3}\right)$

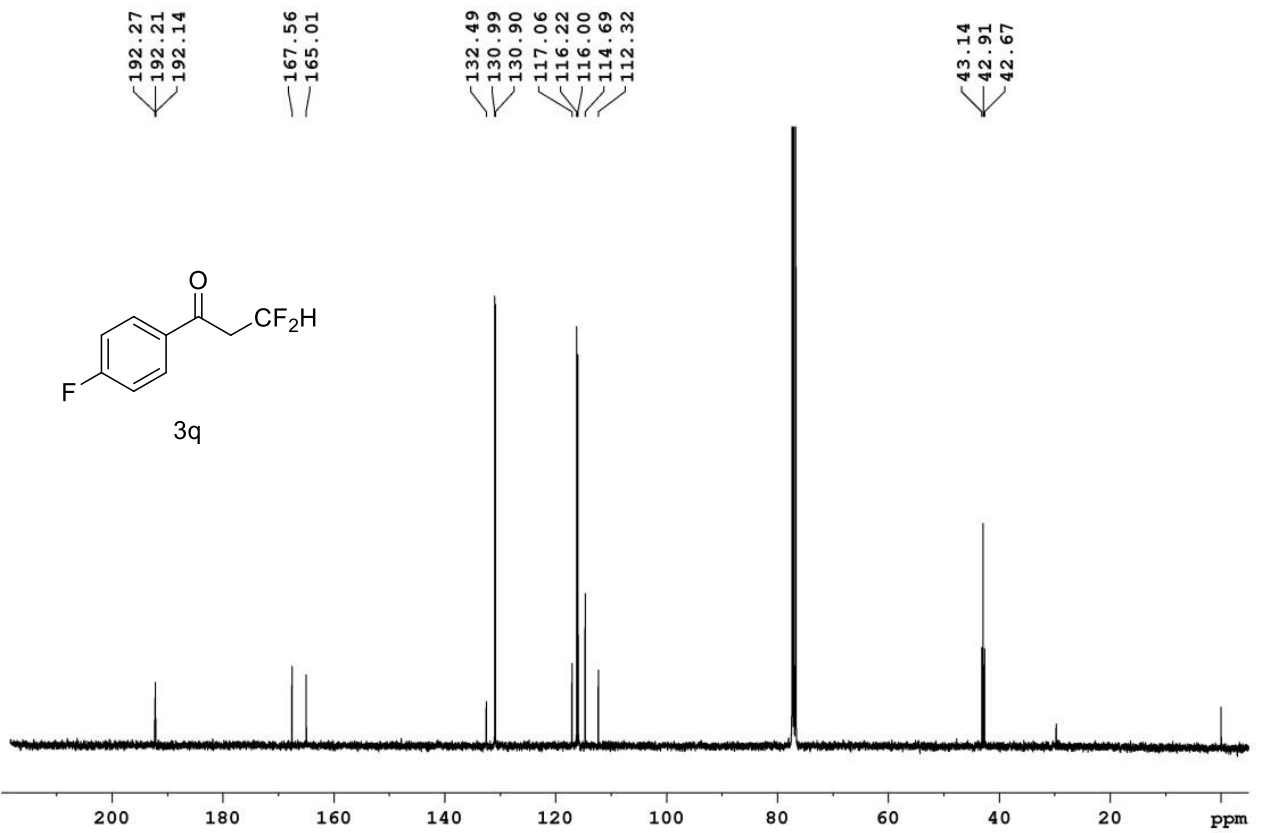

3,3-difluoro-1-(4-fluorophenyl)propan-1-one (3q): ${ }^{19} \mathrm{~F}$ NMR $\left(376.5 \mathrm{MHz}, \mathrm{CDCl}_{3}\right)$

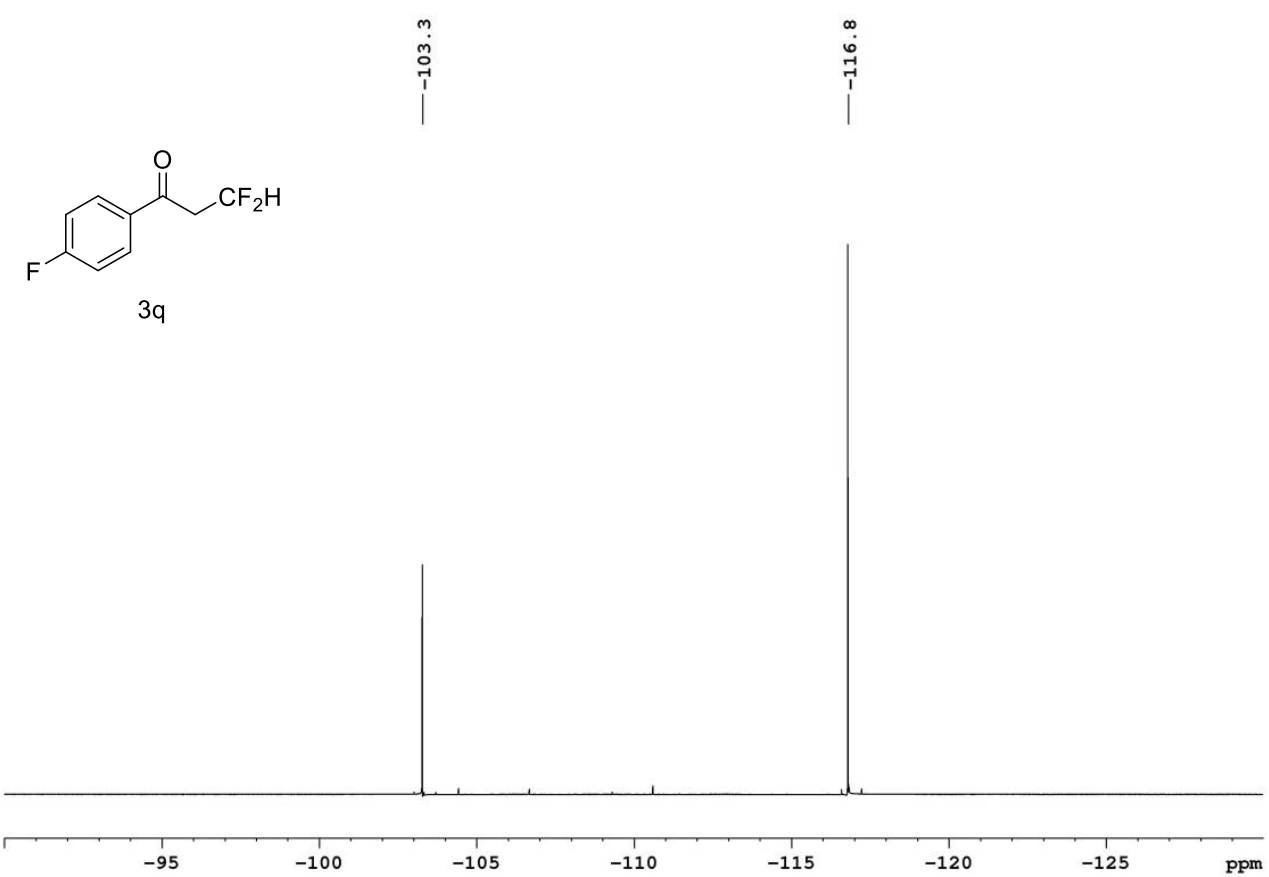


1-(4-chlorophenyl)-3,3-difluoropropan-1-one (3r): ${ }^{1} \mathrm{H}$ NMR (400 MHz, $\left.\mathrm{CDCl}_{3}\right)$

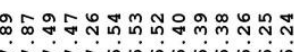

ítíngonomo

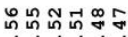

$\underbrace{m m m n}$

$\overbrace{3 r}^{C} C r_{2}$
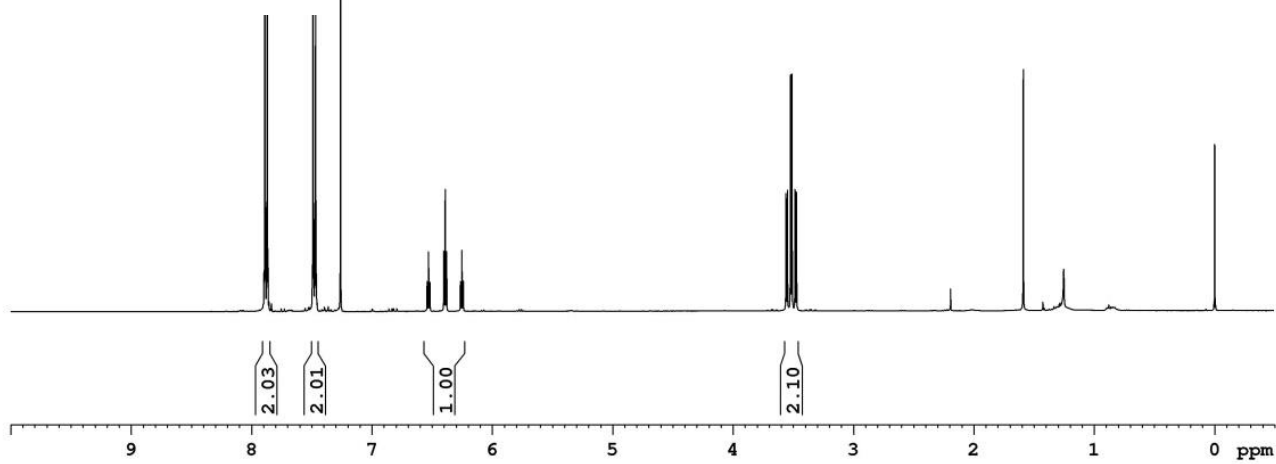

1-(4-chlorophenyl)-3,3-difluoropropan-1-one (3r): $\left.{ }^{13} \mathrm{C} \mathrm{NMR} \mathrm{(100} \mathrm{MHz}, \mathrm{CDCl}_{3}\right)$

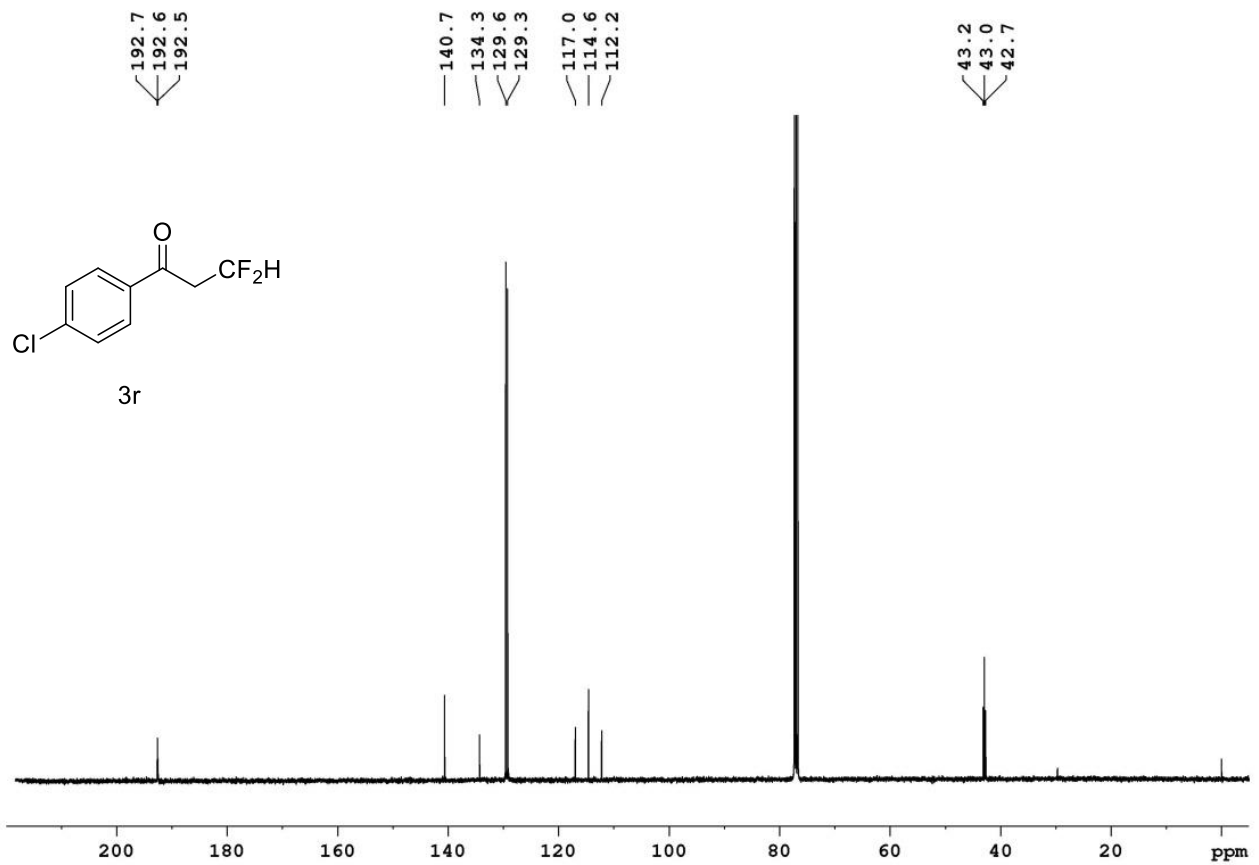


1-(4-chlorophenyl)-3,3-difluoropropan-1-one (3r): $\left.{ }^{19} \mathrm{~F} \mathrm{NMR} \mathrm{(376.5} \mathrm{MHz,} \mathrm{CDCl}_{3}\right)$

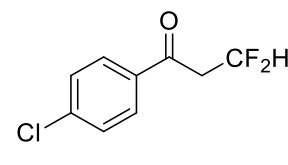

$3 r$

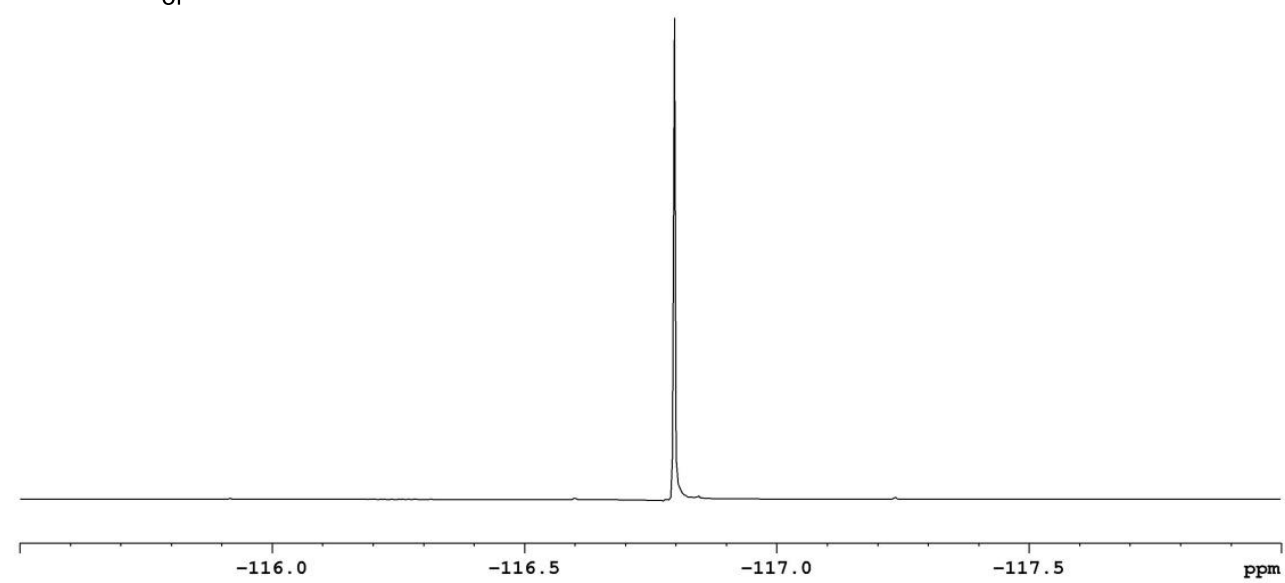

1-(4-bromophenyl)-3,3-difluoropropan-1-one (3s): ${ }^{1} \mathrm{H} \mathrm{NMR}\left(400 \mathrm{MHz}, \mathrm{CDCl}_{3}\right)$

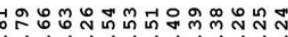

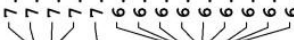

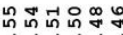

$\underbrace{m m m}$<smiles>O=C(CC(F)F)c1ccc(Br)cc1</smiles>

3s

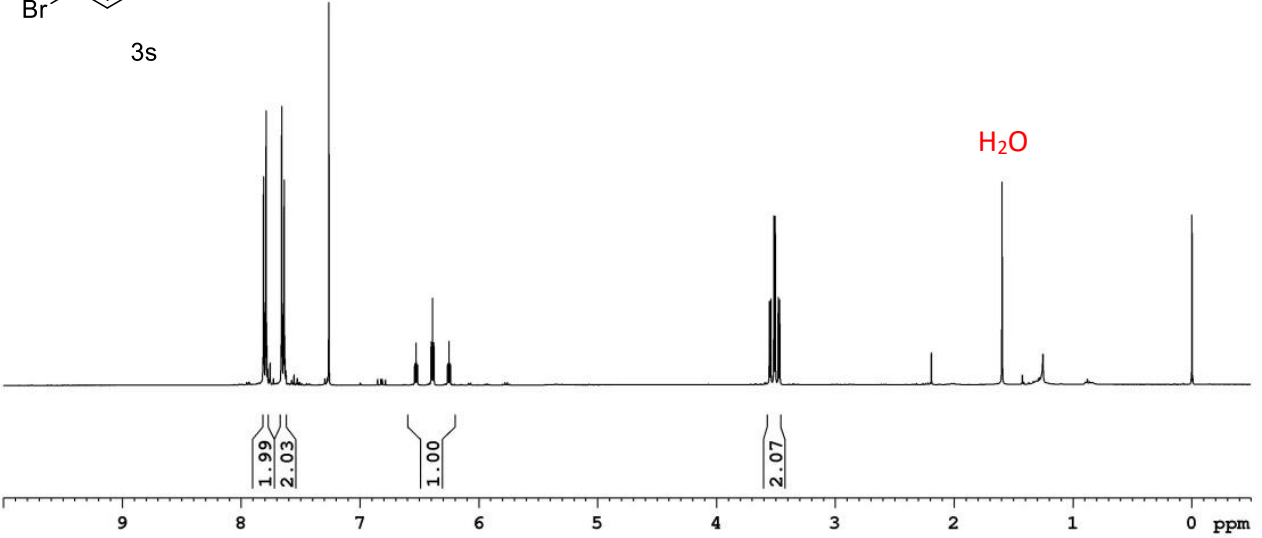

$\mathrm{H}_{2} \mathrm{O}$ 
1-(4-bromophenyl)-3,3-difluoropropan-1-one (3s): $\left.{ }^{13} \mathrm{C} \mathrm{NMR} \mathrm{(100} \mathrm{MHz,} \mathrm{CDCl}_{3}\right)$

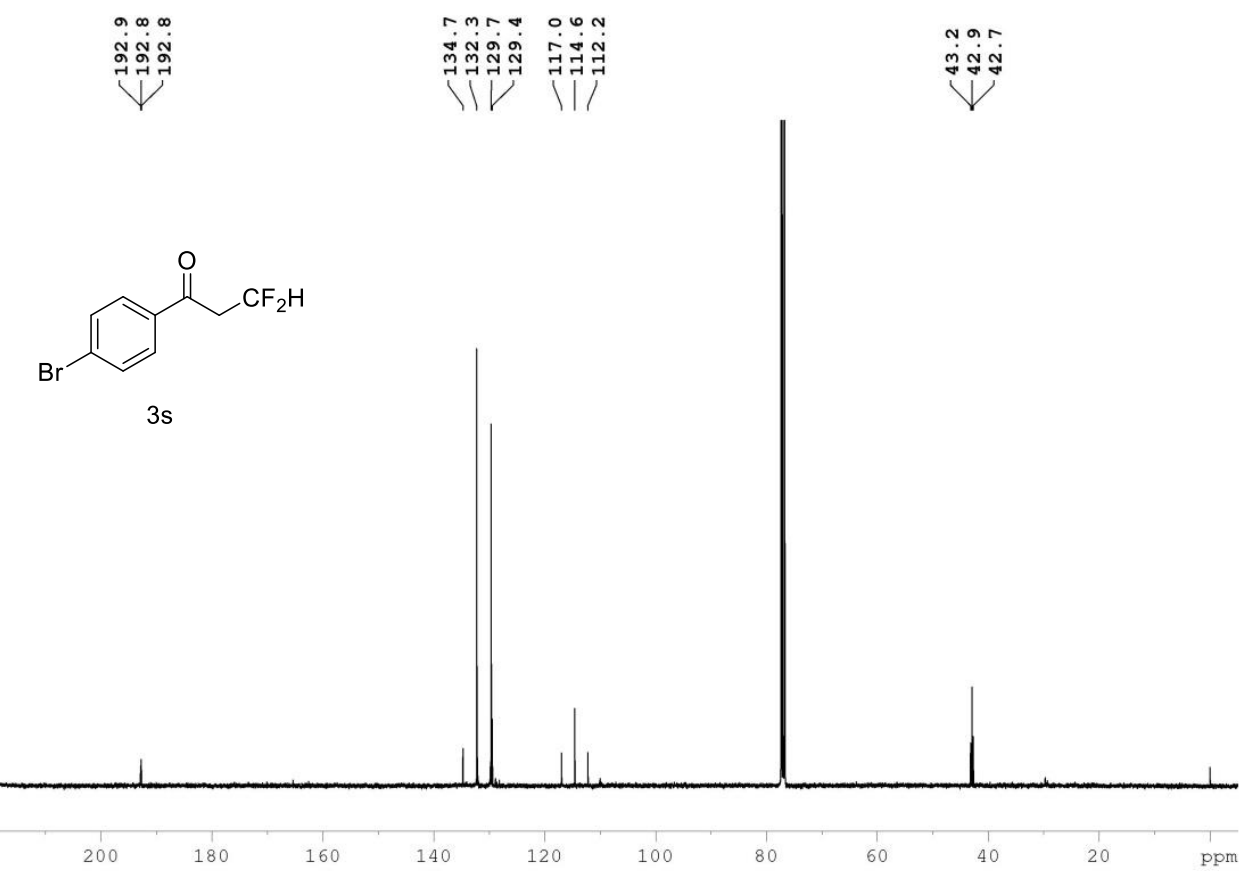

1-(4-bromophenyl)-3,3-difluoropropan-1-one (3s): $\left.{ }^{19} \mathrm{~F} \mathrm{NMR} \mathrm{(376.5} \mathrm{MHz}, \mathrm{CDCl}_{3}\right)$

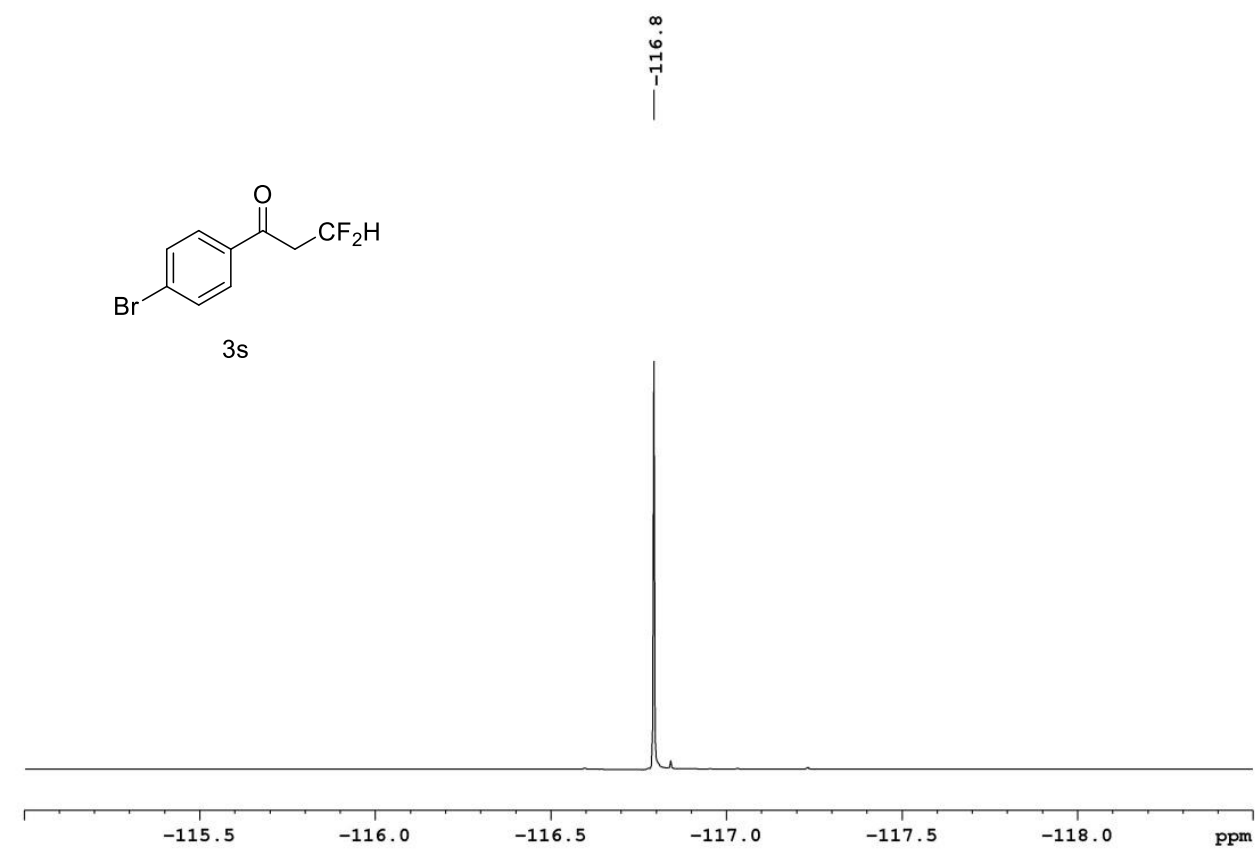


2-(difluoromethyl)-3,4-dihydronaphthalen-1(2H)-one (3u): ${ }^{1} \mathrm{H} \mathrm{NMR}\left(400 \mathrm{MHz}, \mathrm{CDCl}_{3}\right)$

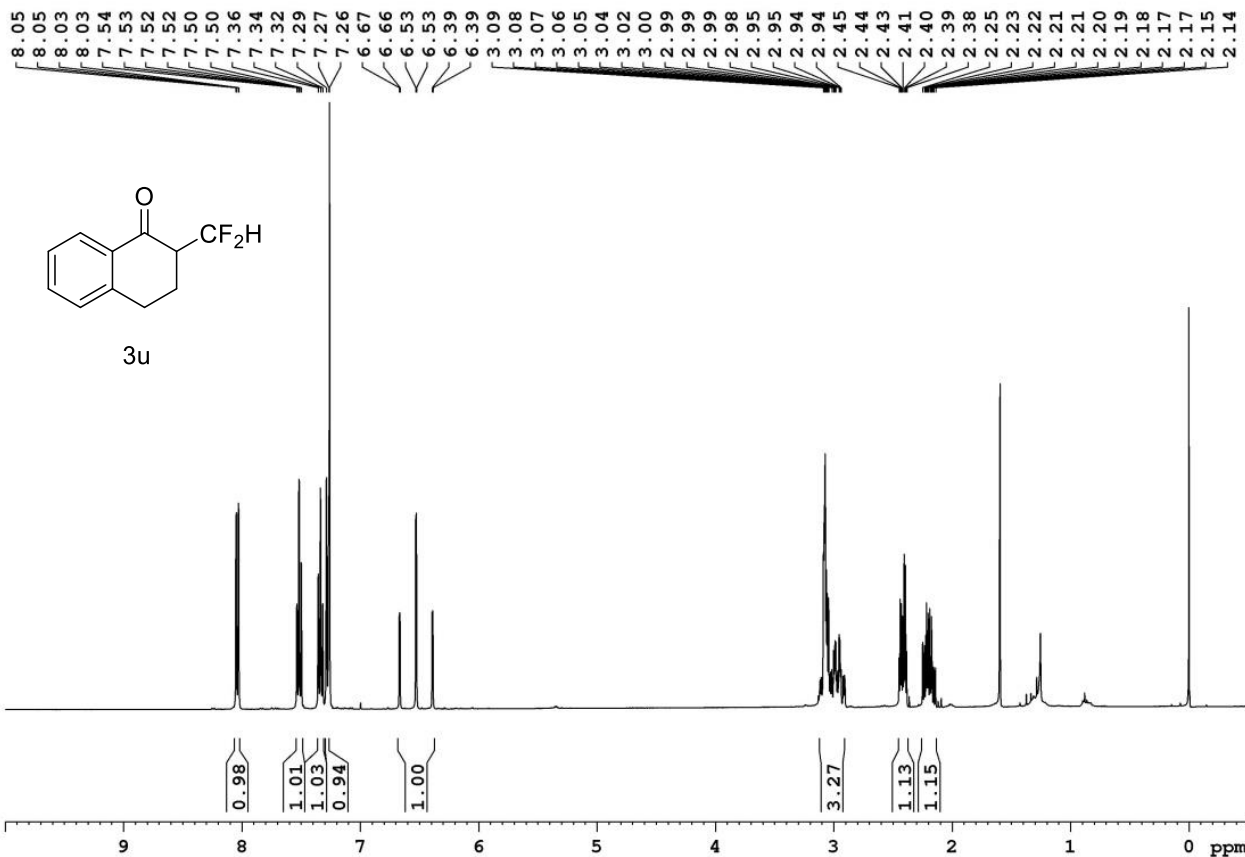

2-(difluoromethyl)-3,4-dihydronaphthalen-1(2H)-one (3u): ${ }^{13} \mathrm{C} \mathrm{NMR}\left(100 \mathrm{MHz}, \mathrm{CDCl}_{3}\right)$

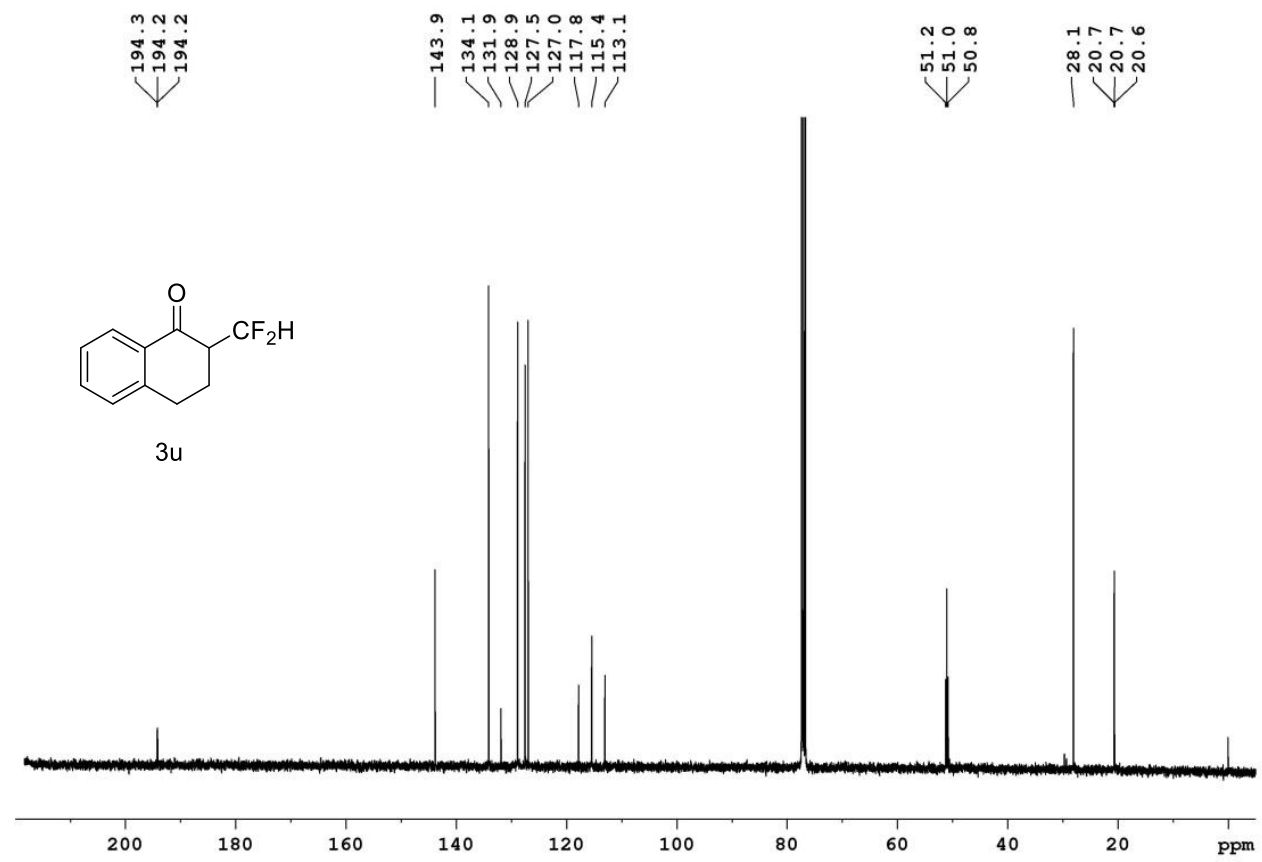


2-(difluoromethyl)-3,4-dihydronaphthalen-1(2H)-one (3u): ${ }^{19} \mathrm{~F} \mathrm{NMR}\left(376.5 \mathrm{MHz}, \mathrm{CDCl}_{3}\right)$
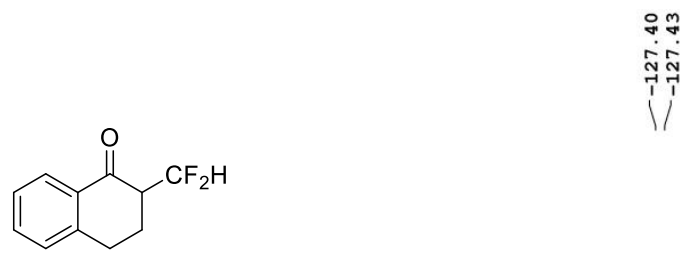

$3 u$

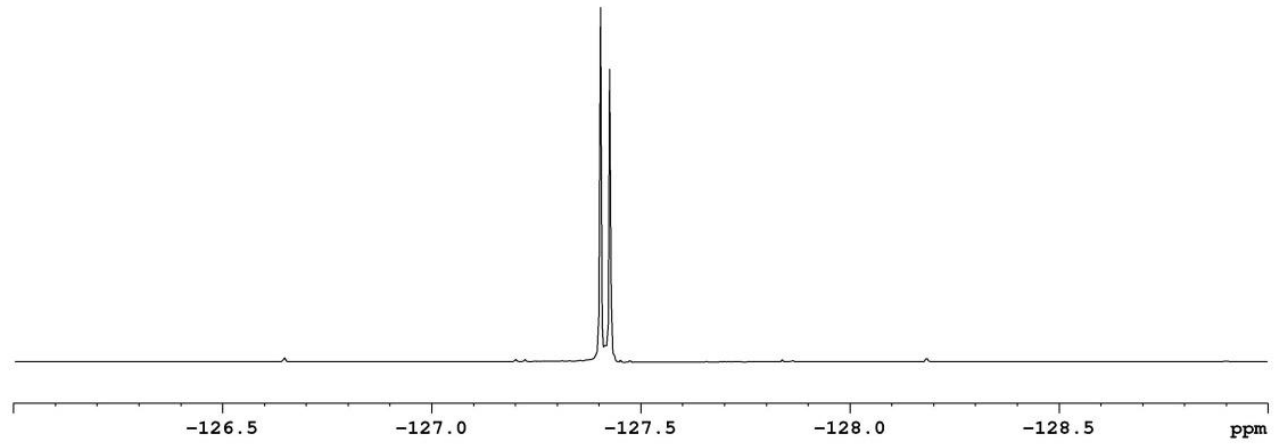

2-(difluoromethyl)-3,4-dihydronaphthalen-1(2H)-one (3u): (HRMS)

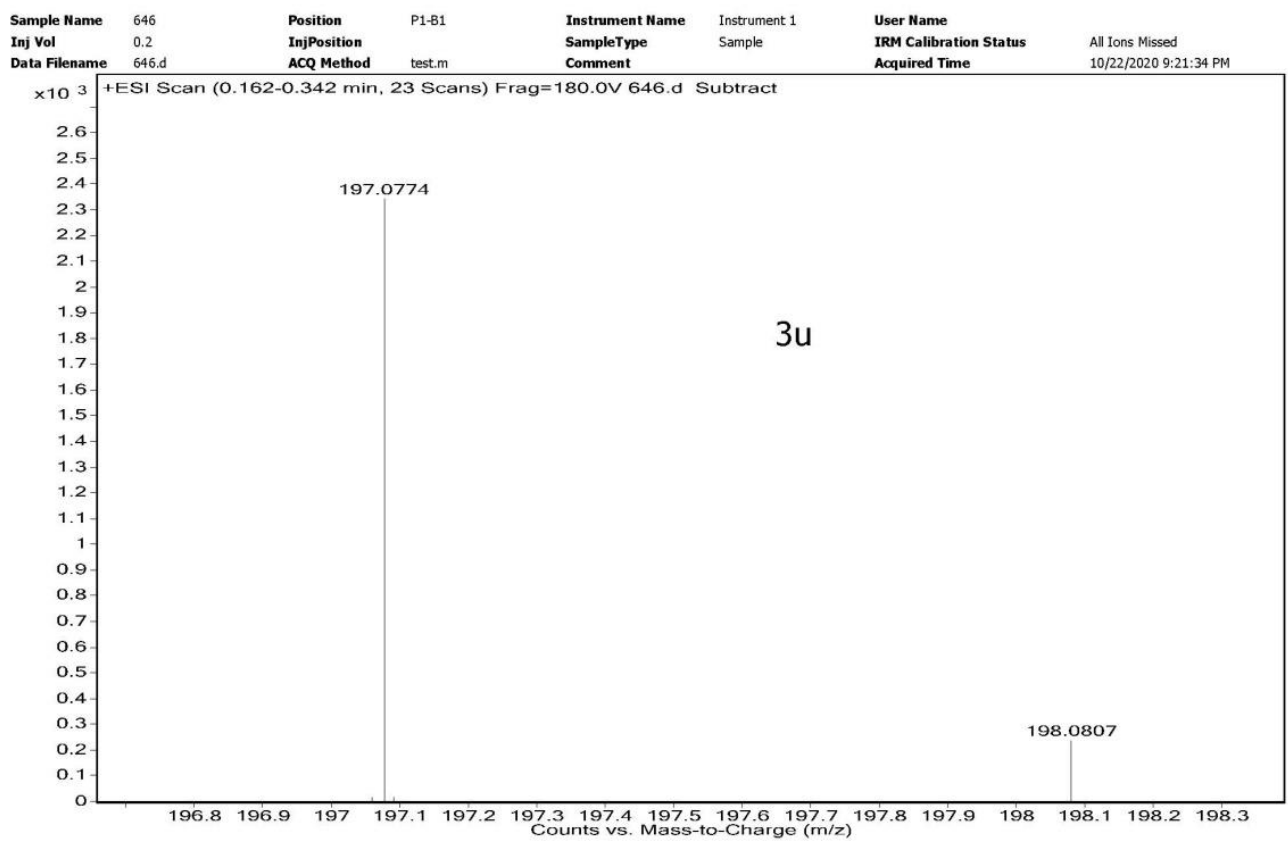


3,3-difluoro-2-methyl-1-(p-tolyl)propan-1-one (3v): ${ }^{1} \mathrm{H} \mathrm{NMR}\left(400 \mathrm{MHz}, \mathrm{CDCl}_{3}\right)$
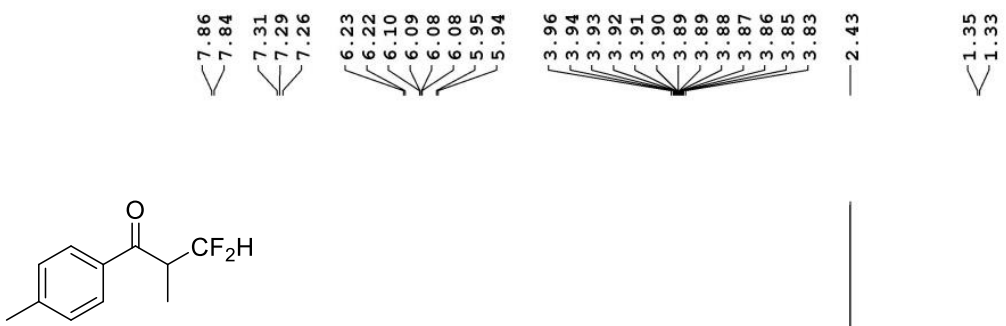

$3 v$

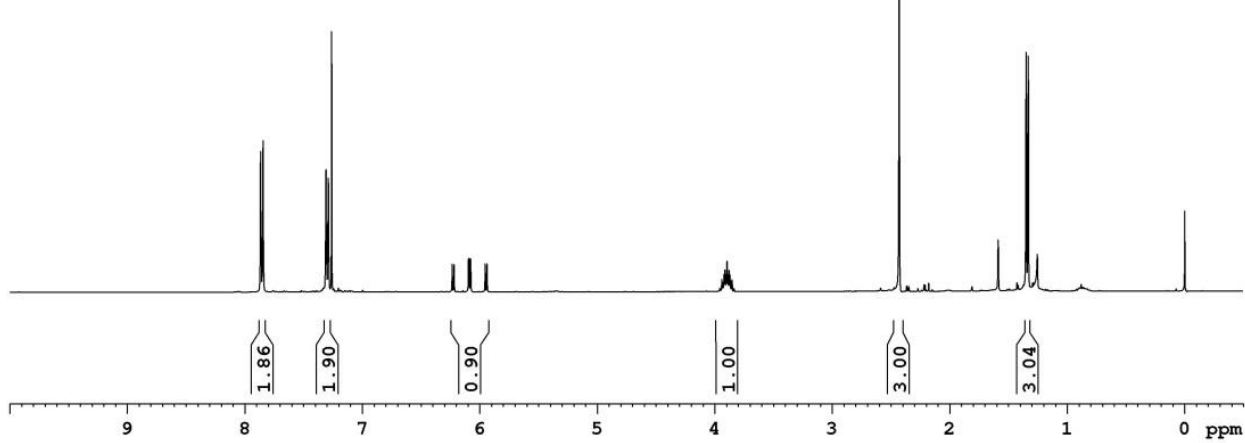

3,3-difluoro-2-methyl-1-(p-tolyl)propan-1-one (3v): ${ }^{13} \mathrm{C} \mathrm{NMR}\left(100 \mathrm{MHz}, \mathrm{CDCl}_{3}\right)$

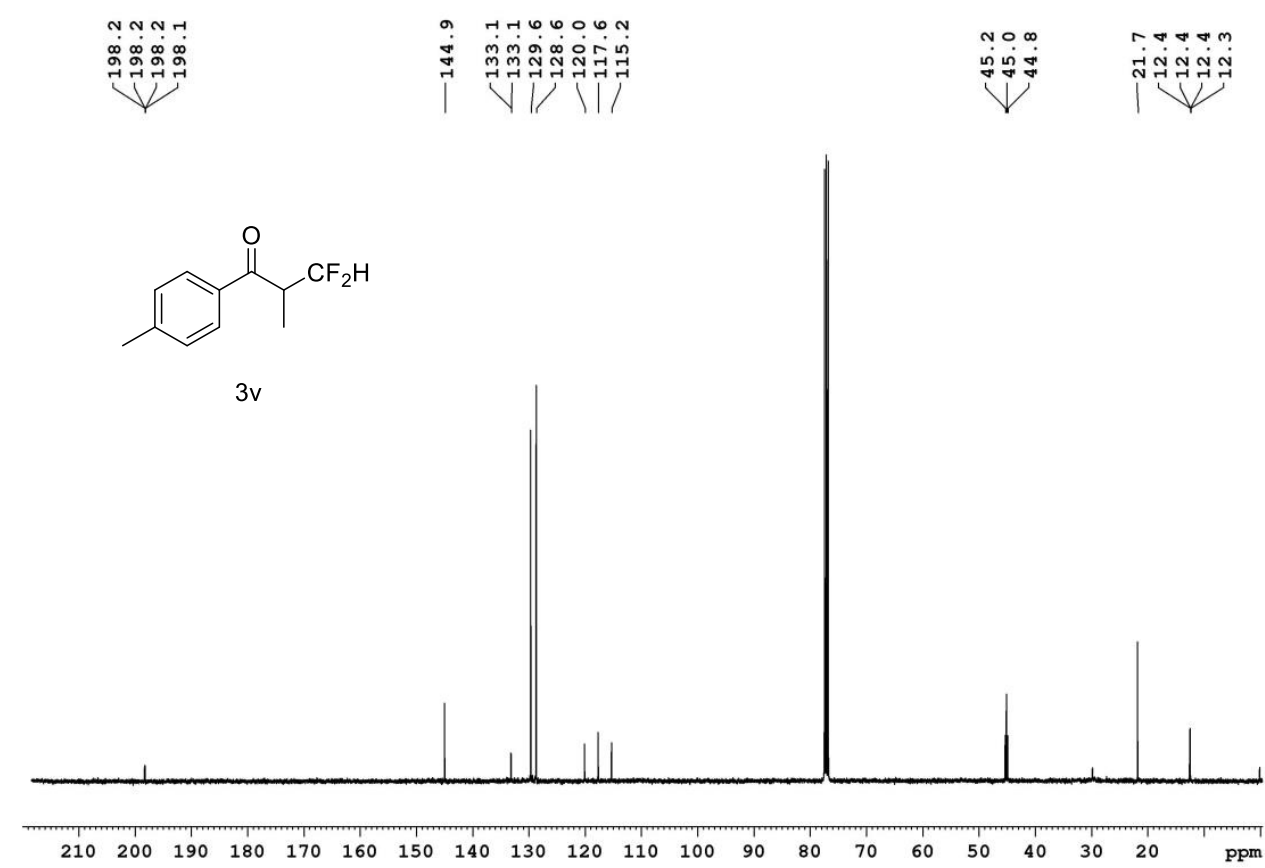


3,3-difluoro-2-methyl-1-(p-tolyl)propan-1-one (3v): ${ }^{19} \mathrm{~F} \mathrm{NMR}\left(376.5 \mathrm{MHz}, \mathrm{CDCl}_{3}\right)$
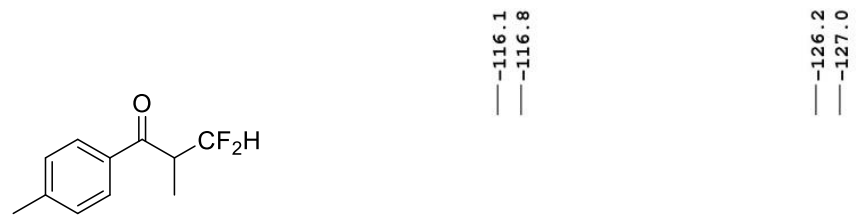

$3 v$

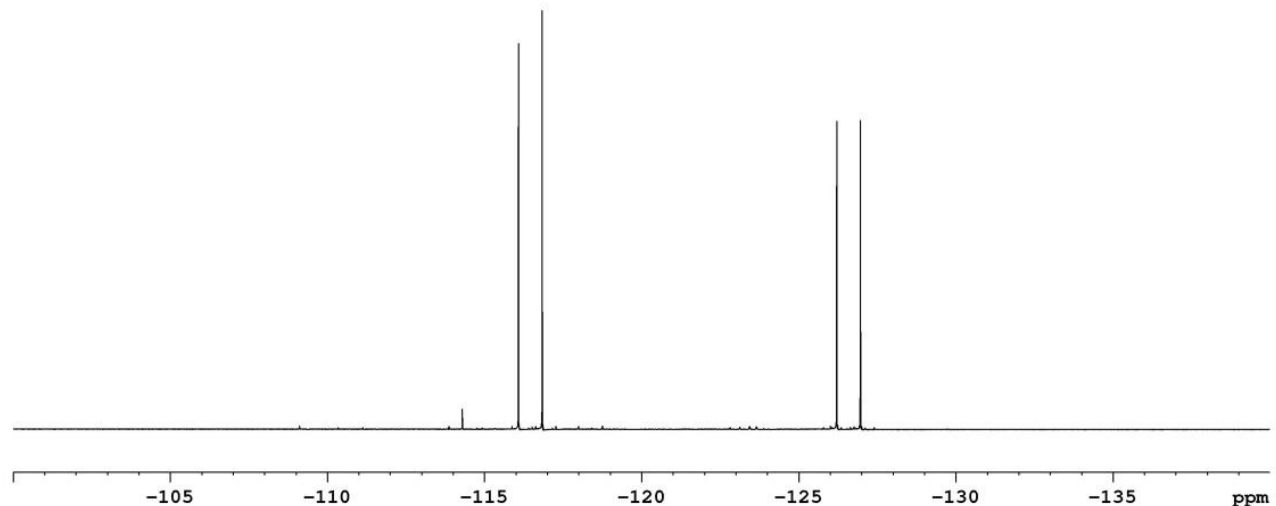

3,3-difluoro-2-methyl-1-(p-tolyl)propan-1-one (3v): (HRMS)

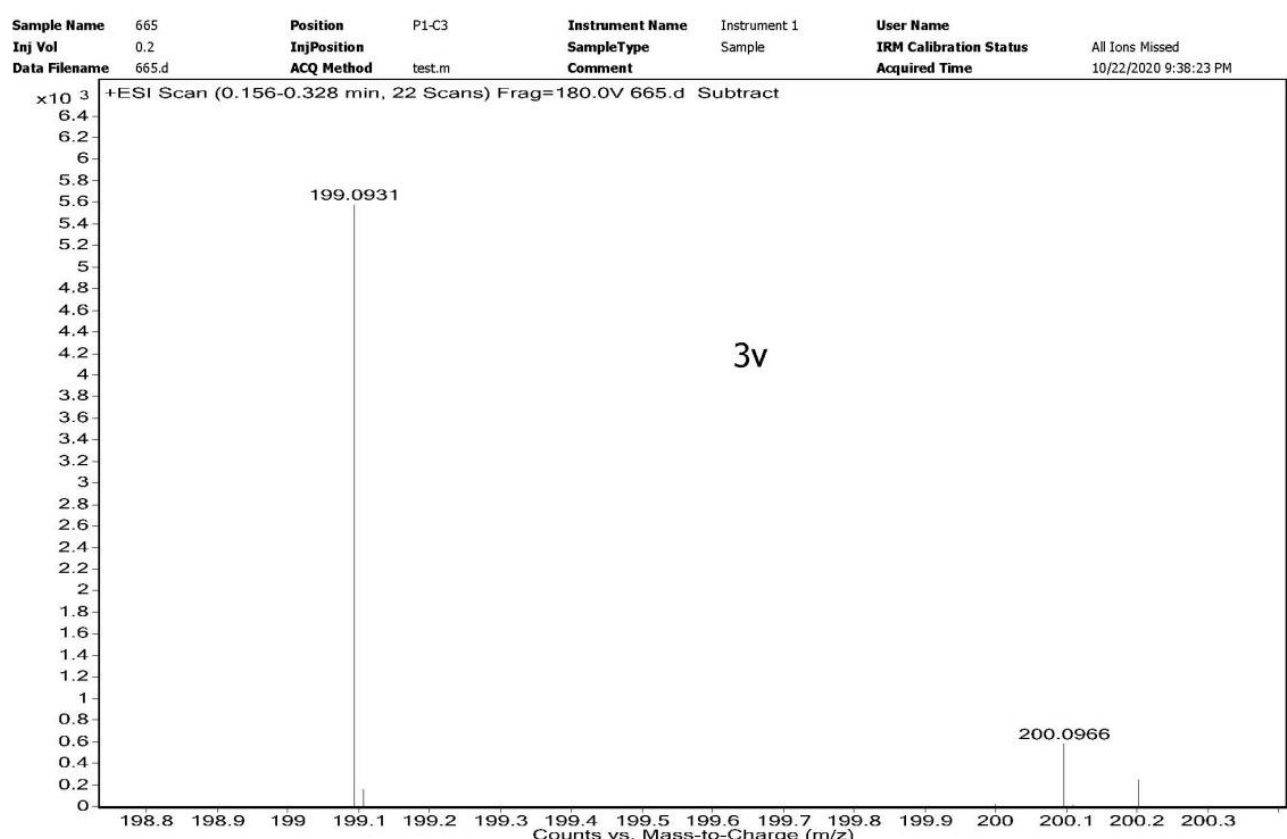


3,3-difluoro-1-(thiophen-3-yl)propan-1-one (3w): ${ }^{1} \mathrm{H}$ NMR $\left(400 \mathrm{MHz}, \mathrm{CDCl}_{3}\right)$

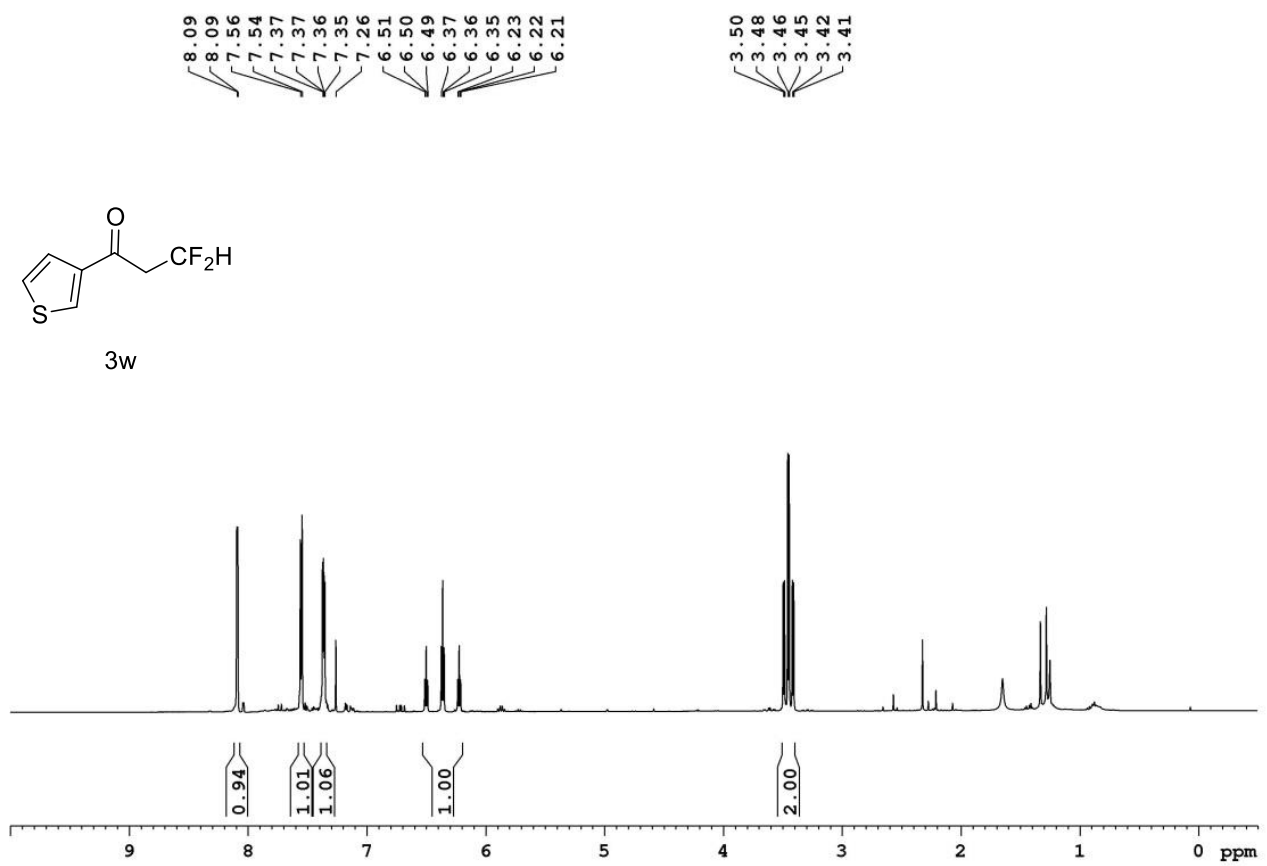

3,3-difluoro-1-(thiophen-3-yl)propan-1-one (3w): ${ }^{13} \mathrm{C}$ NMR (100 MHz, $\left.\mathrm{CDCl}_{3}\right)$

\begin{tabular}{|c|c|c|c|c|}
\hline$\underset{\infty}{\infty}$ & $\frac{n}{4}$ & 惫突芯 & 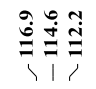 & 落点 \\
\hline
\end{tabular}

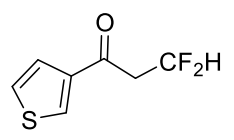

$3 w$

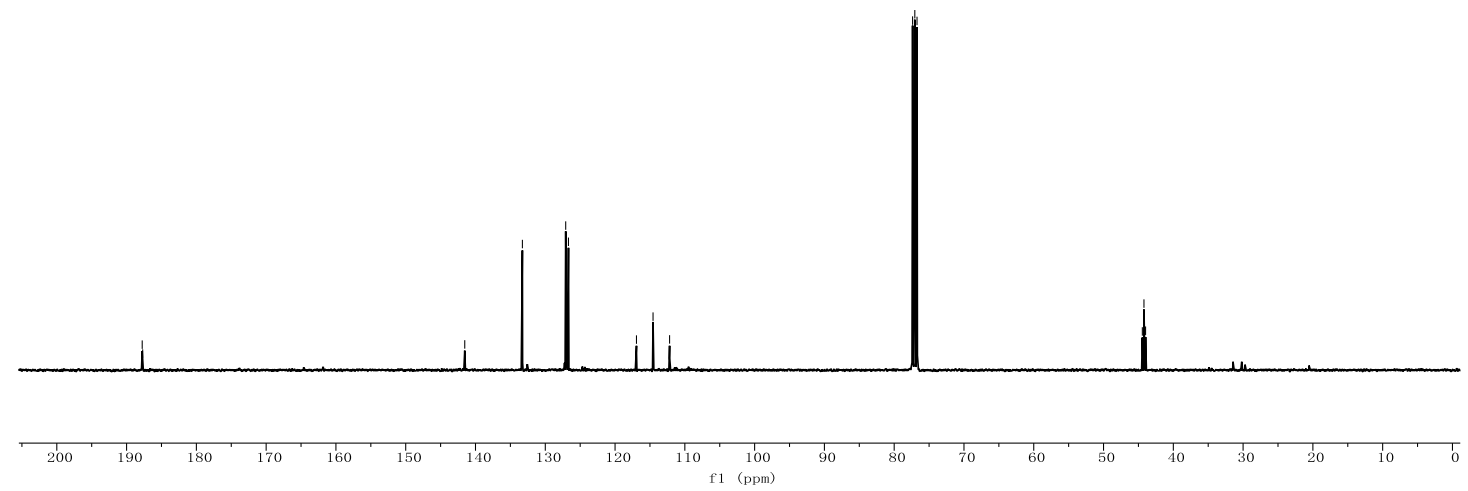


3,3-difluoro-1-(thiophen-3-yl)propan-1-one (3w): ${ }^{19} \mathrm{~F}$ NMR (376.5 MHz, $\left.\mathrm{CDCl}_{3}\right)$

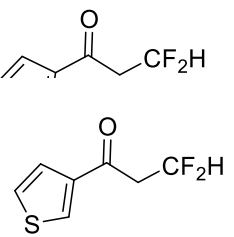

$3 w$

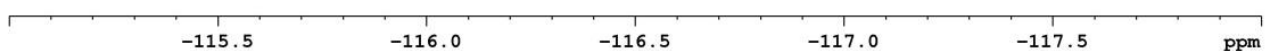

3,3-difluoro-1-(1-tosyl-1H-indol-3-yl)propan-1-one (3x): ${ }^{1} \mathrm{H} \mathrm{NMR}\left(400 \mathrm{MHz}, \mathrm{CDCl}_{3}\right)$

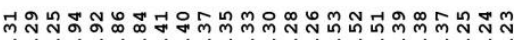
$\underbrace{\infty}$

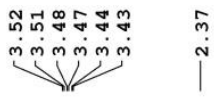<smiles>O=C(CC(F)F)c1cn([12F])c2ccccc12</smiles>

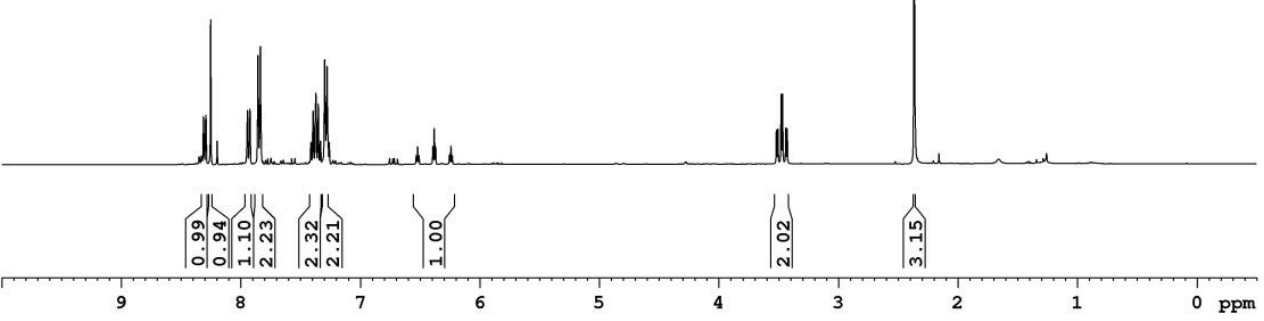


3,3-difluoro-1-(1-tosyl-1H-indol-3-yl)propan-1-one (3x): ${ }^{13} \mathrm{C}$ NMR (100 MHz, $\left.\mathrm{CDCl}_{3 \mathrm{e}}\right)$

\begin{tabular}{|c|c|c|c|}
\hline 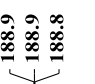 & 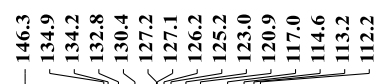 & 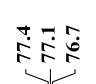 & 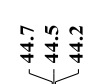 \\
\hline
\end{tabular}
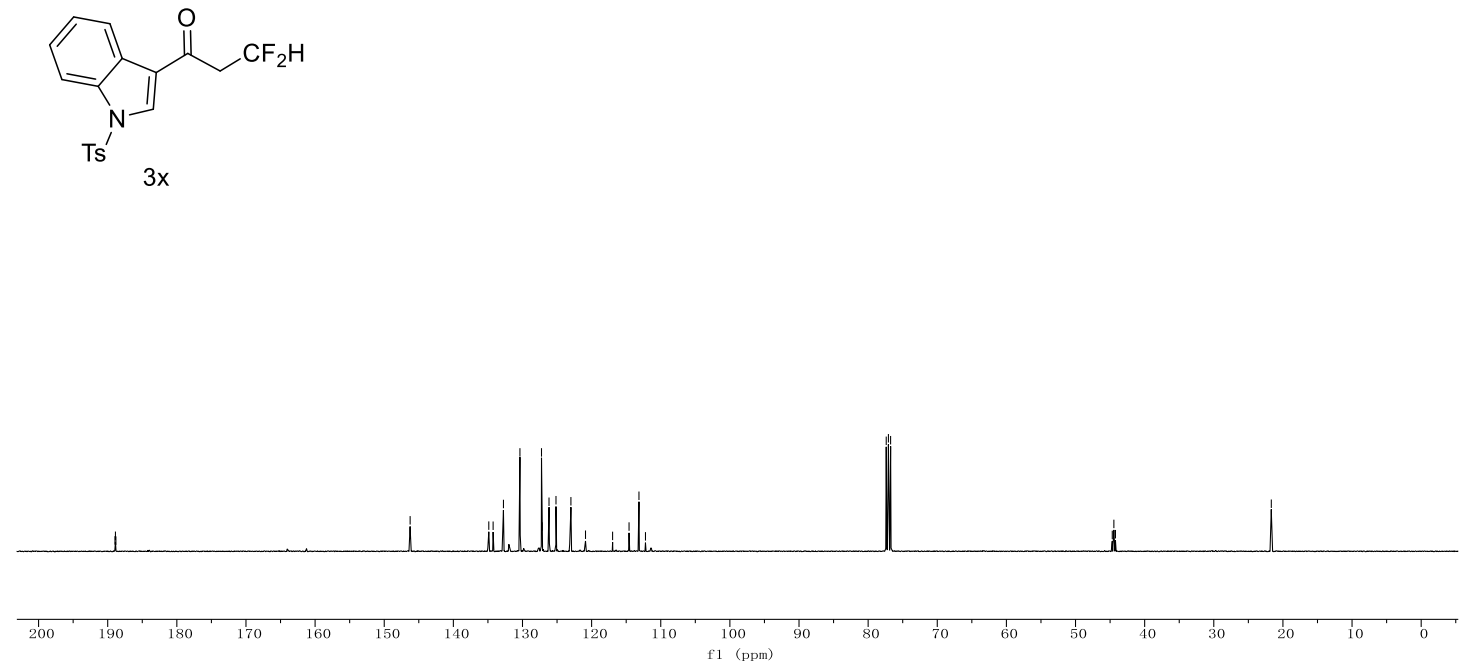

3,3-difluoro-1-(1-tosyl-1H-indol-3-yl)propan-1-one (3x): ${ }^{19} \mathrm{~F}$ NMR (376.5 MHz, $\left.\mathrm{CDCl}_{3}\right)$

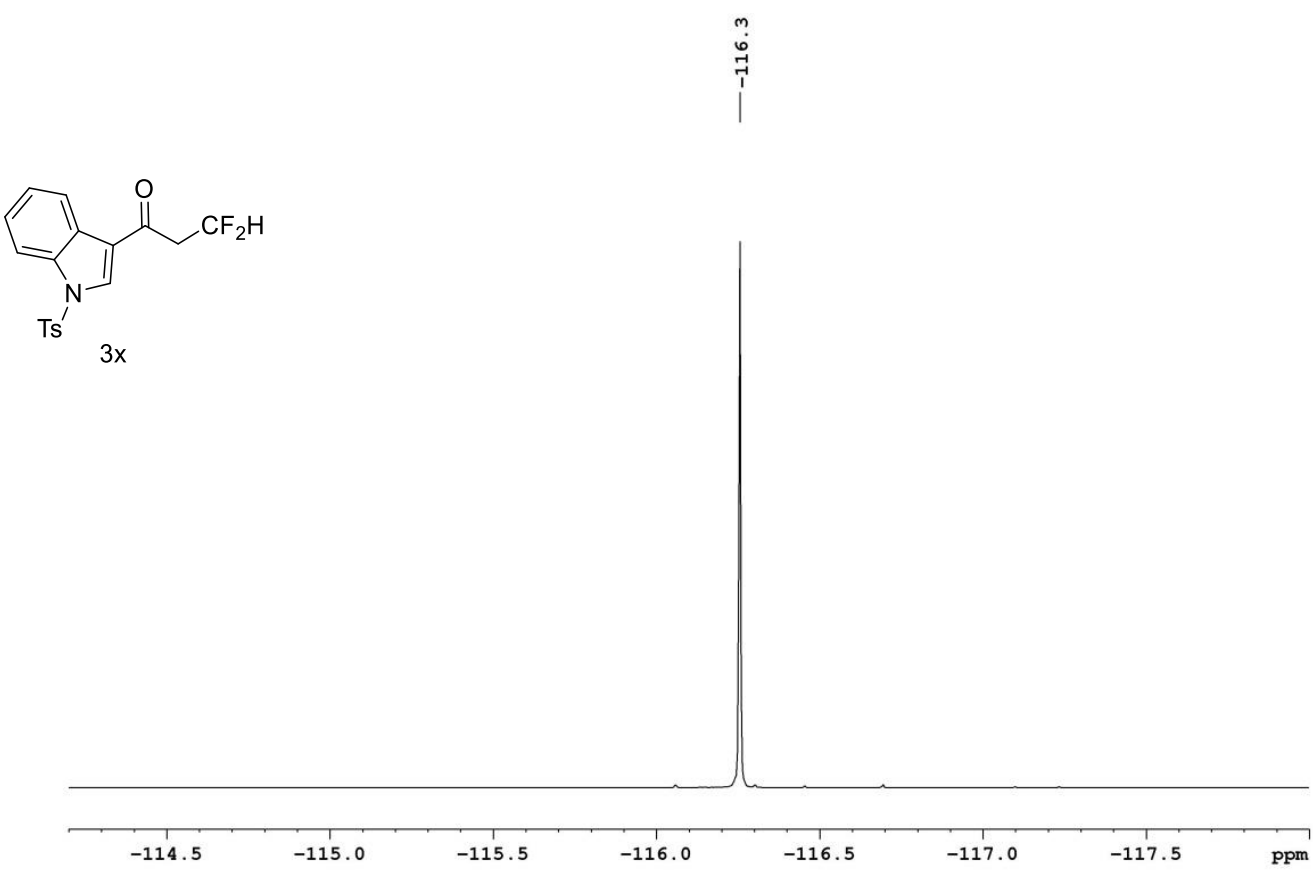


1-(4-ethylphenyl)-3,3-difluoropropan-1-one (3z): ${ }^{1} \mathrm{H} \mathrm{NMR}\left(400 \mathrm{MHz}, \mathrm{CDCl}_{3}\right)$

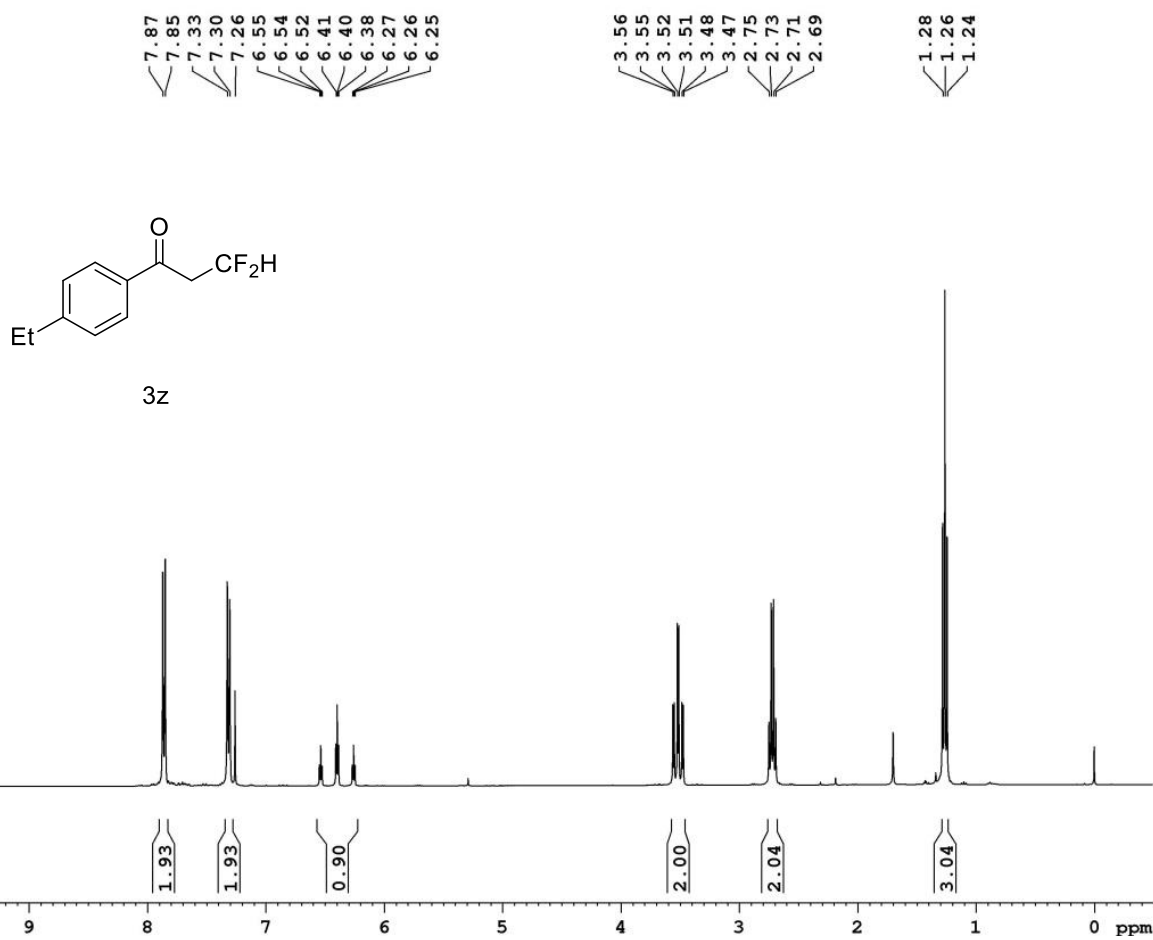

1-(4-ethylphenyl)-3,3-difluoropropan-1-one (3z): $\left.{ }^{13} \mathrm{C} \mathrm{NMR} \mathrm{(100} \mathrm{MHz}, \mathrm{CDCl}_{3}\right)$

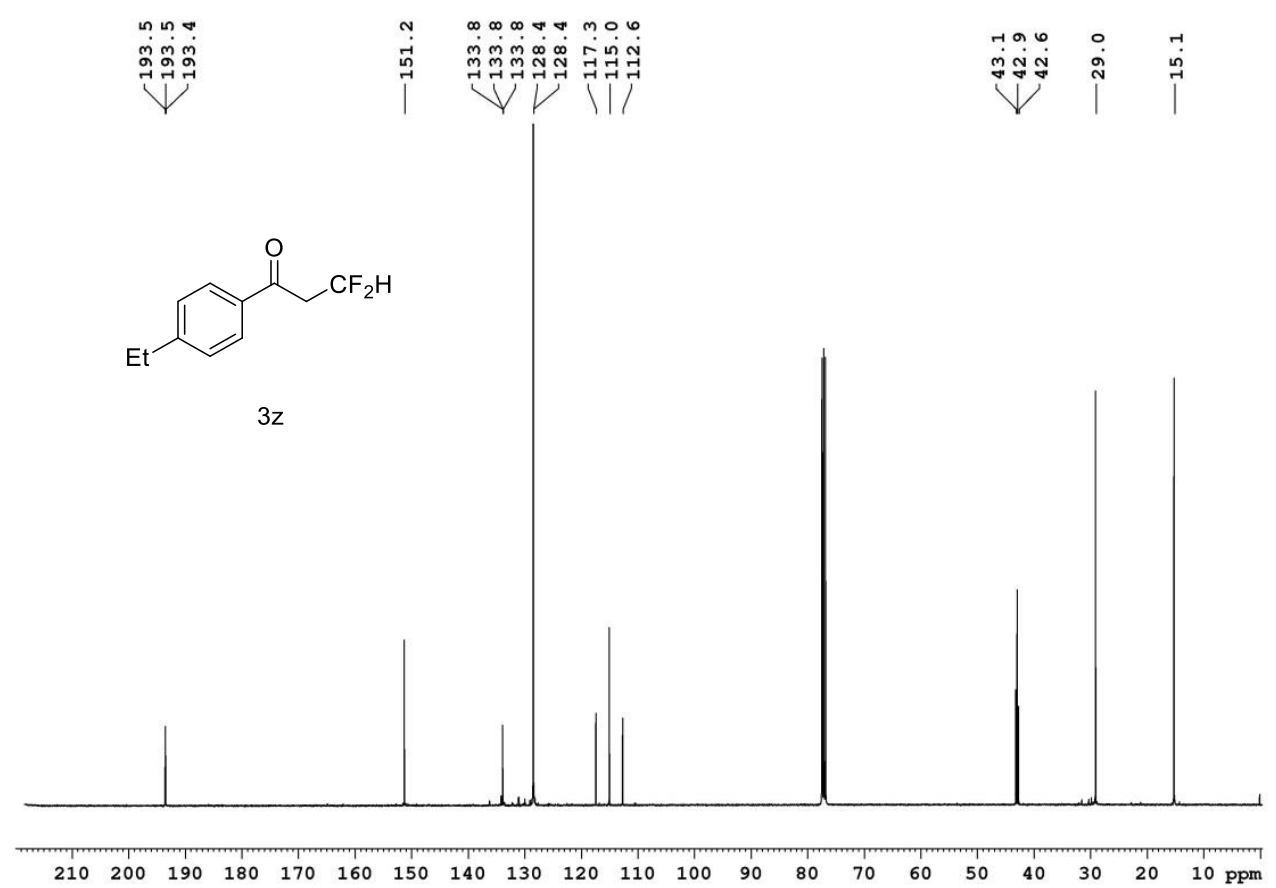


1-(4-ethylphenyl)-3,3-difluoropropan-1-one (3z): ${ }^{19} \mathrm{~F}$ NMR (376.5 MHz, $\left.\mathrm{CDCl}_{3}\right)$

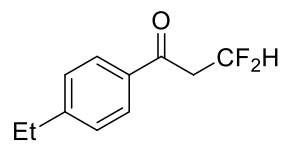

$3 z$

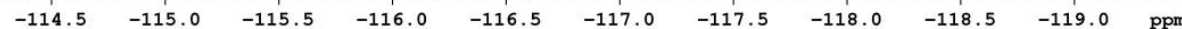

1-(4-ethylphenyl)-3,3-difluoropropan-1-one (3z): (HRMS)

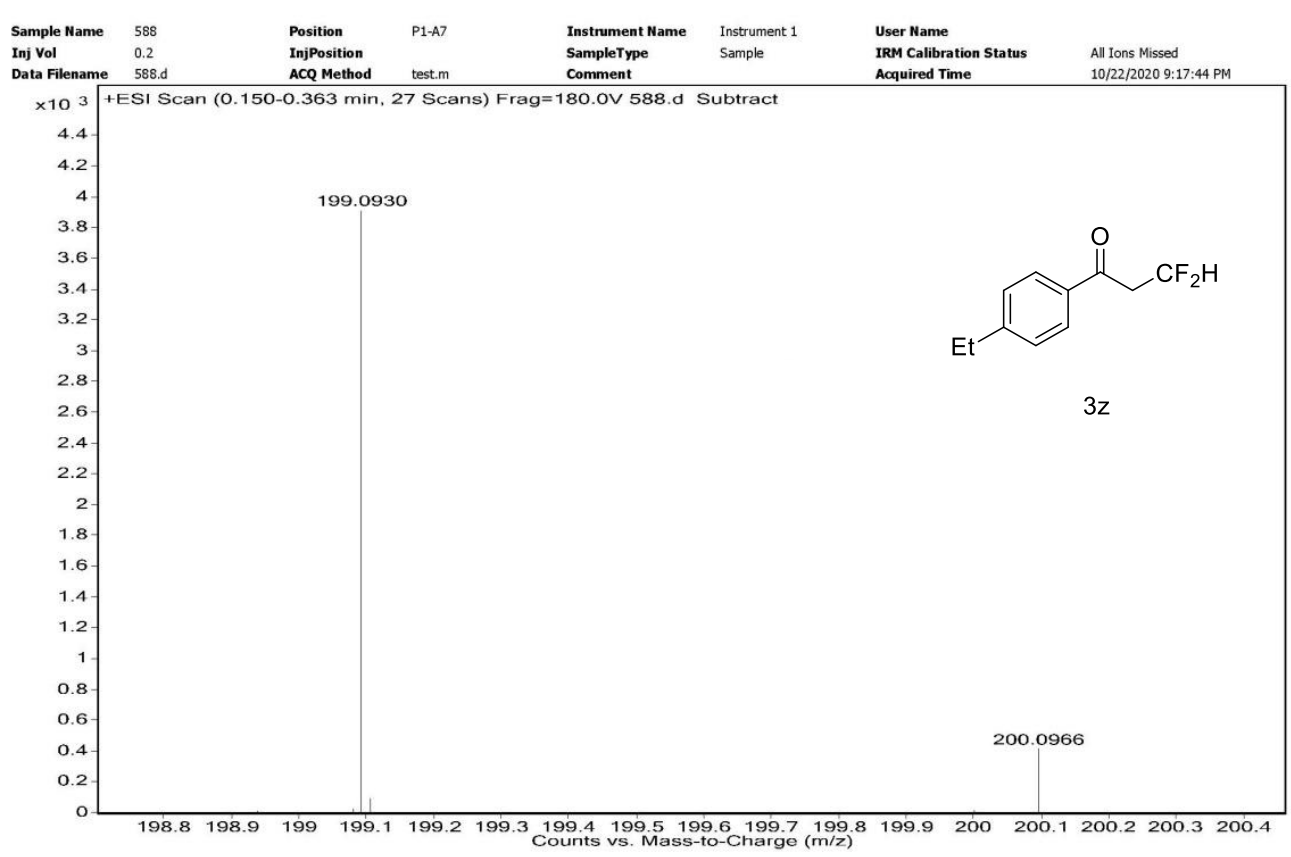


3,3-difluoro-1-(p-tolyl)propan-1-ol (4): ${ }^{1} \mathrm{H}$ NMR (400 MHz, $\mathrm{CDCl}_{3}$ )

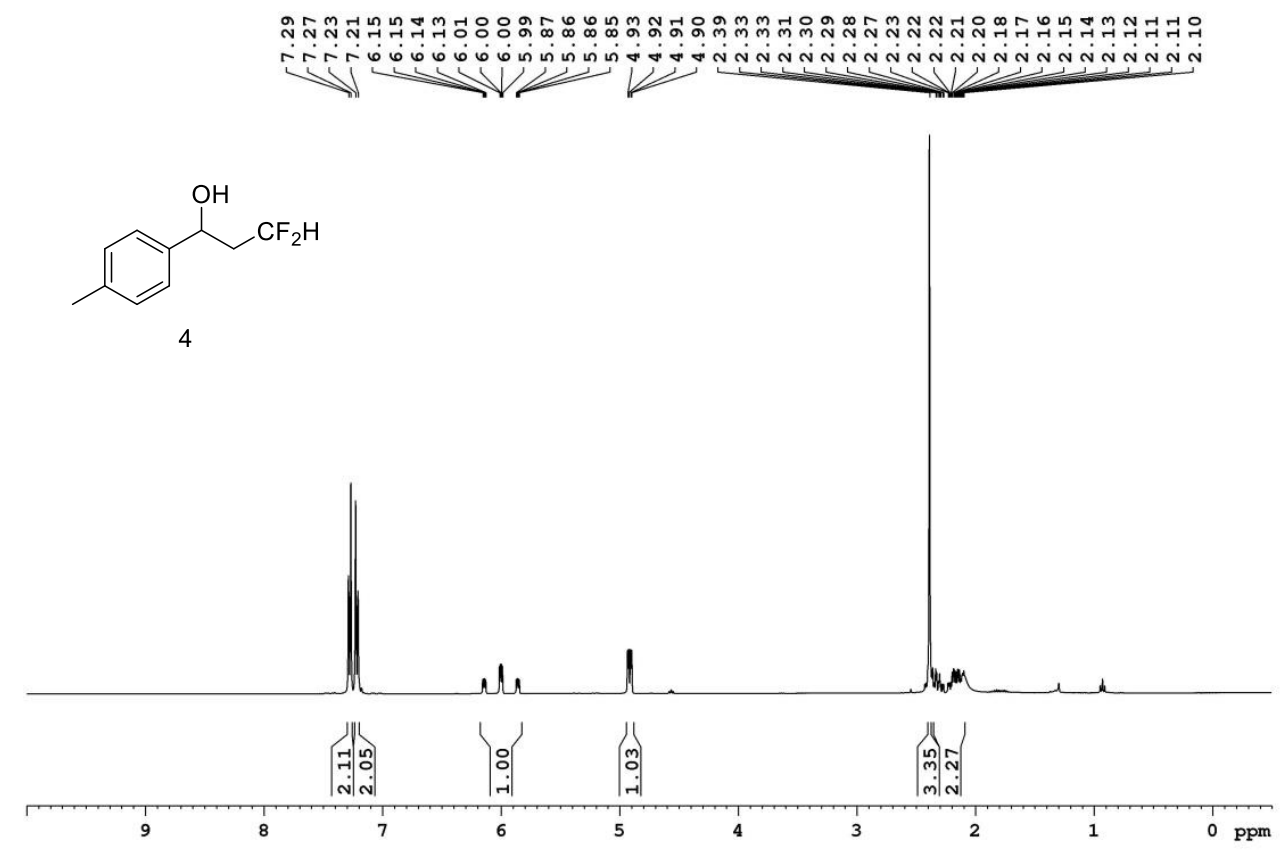

3,3-difluoro-1-(p-tolyl)propan-1-ol (4): ${ }^{13} \mathrm{C} \mathrm{NMR}\left(100 \mathrm{MHz}, \mathrm{CDCl}_{3}\right)$
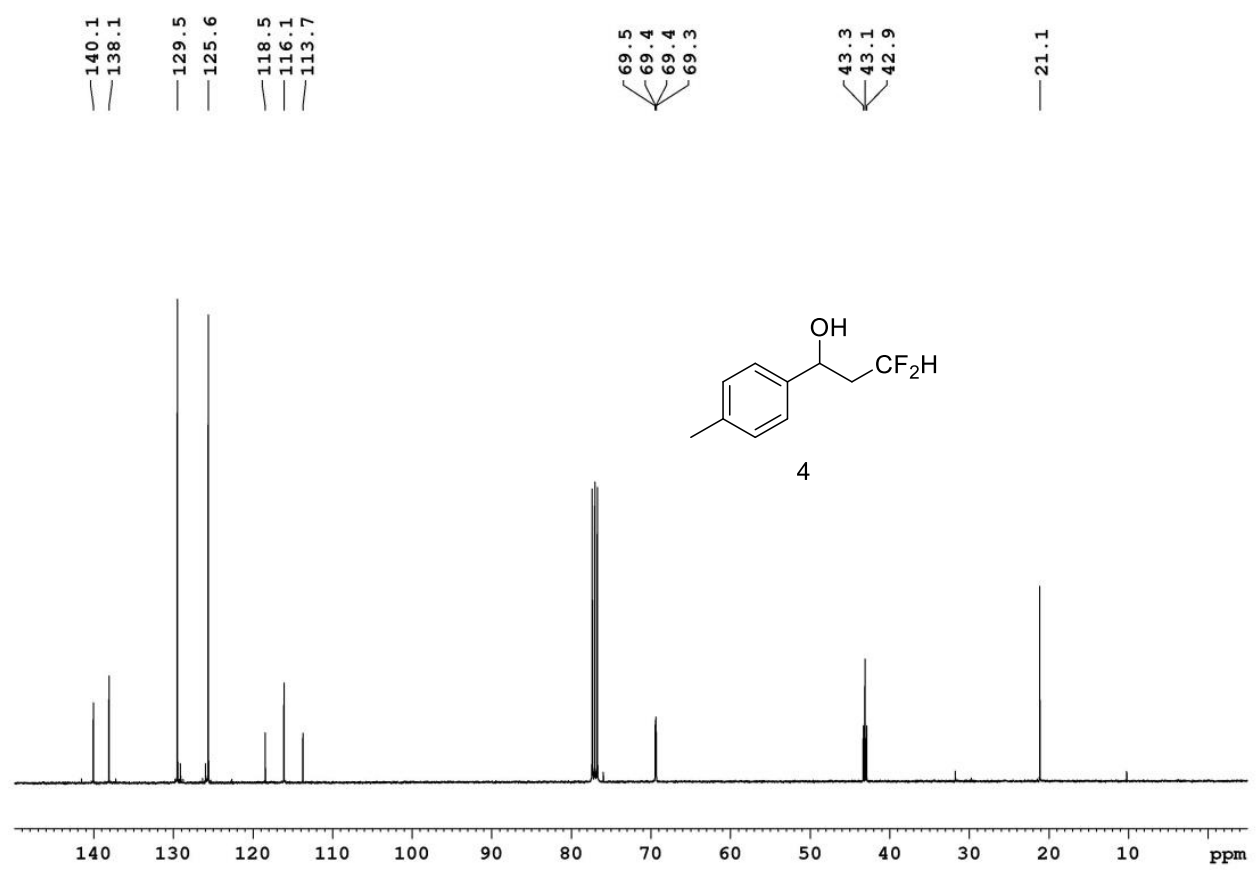
3,3-difluoro-1-(p-tolyl)propan-1-ol (4): ${ }^{19} \mathrm{~F} \mathrm{NMR} \mathrm{(376.5} \mathrm{MHz,} \mathrm{CDCl}_{3}$ )
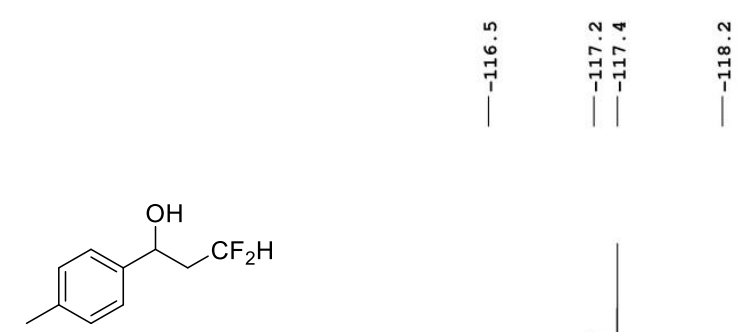

4

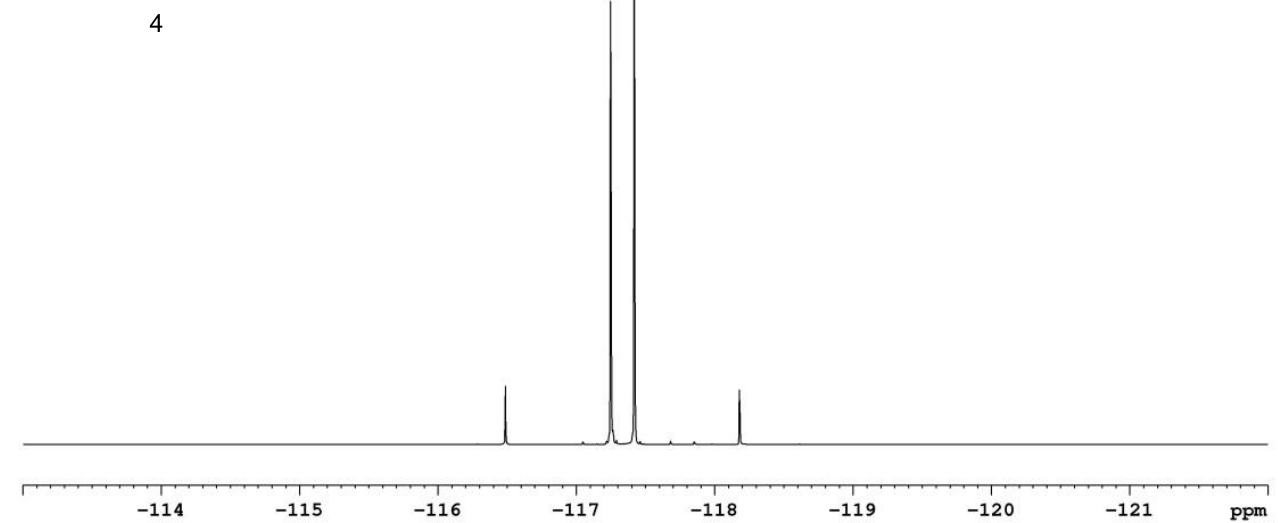

3,3-difluoro-1-(p-tolyl)propan-1-ol (4): (HRMS)

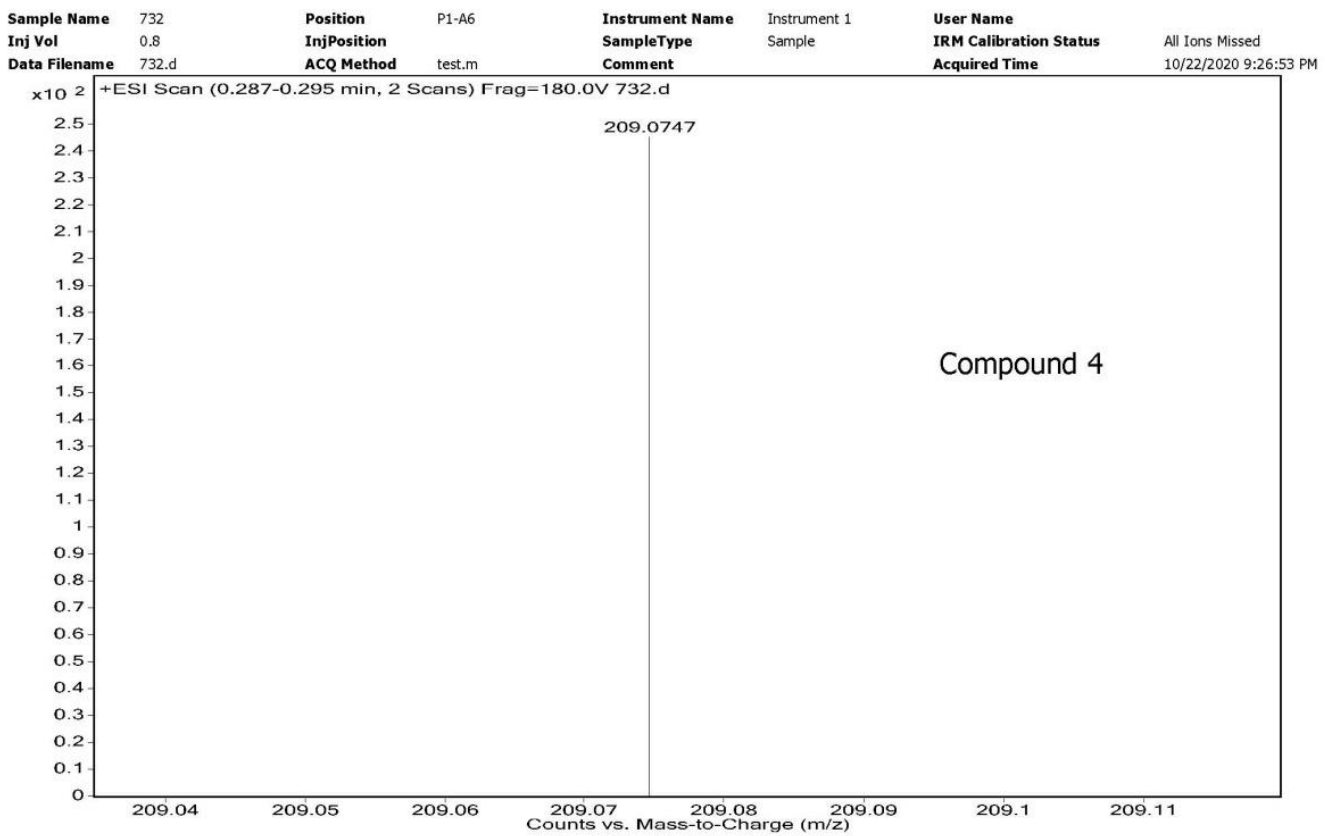


4,4-difluoro-2-(p-tolyl)-2-((trimethylsilyl)oxy)butanenitrile (5): ${ }^{1} \mathrm{H}$ NMR (400 MHz, $\left.\mathrm{CDCl}_{3}\right)$

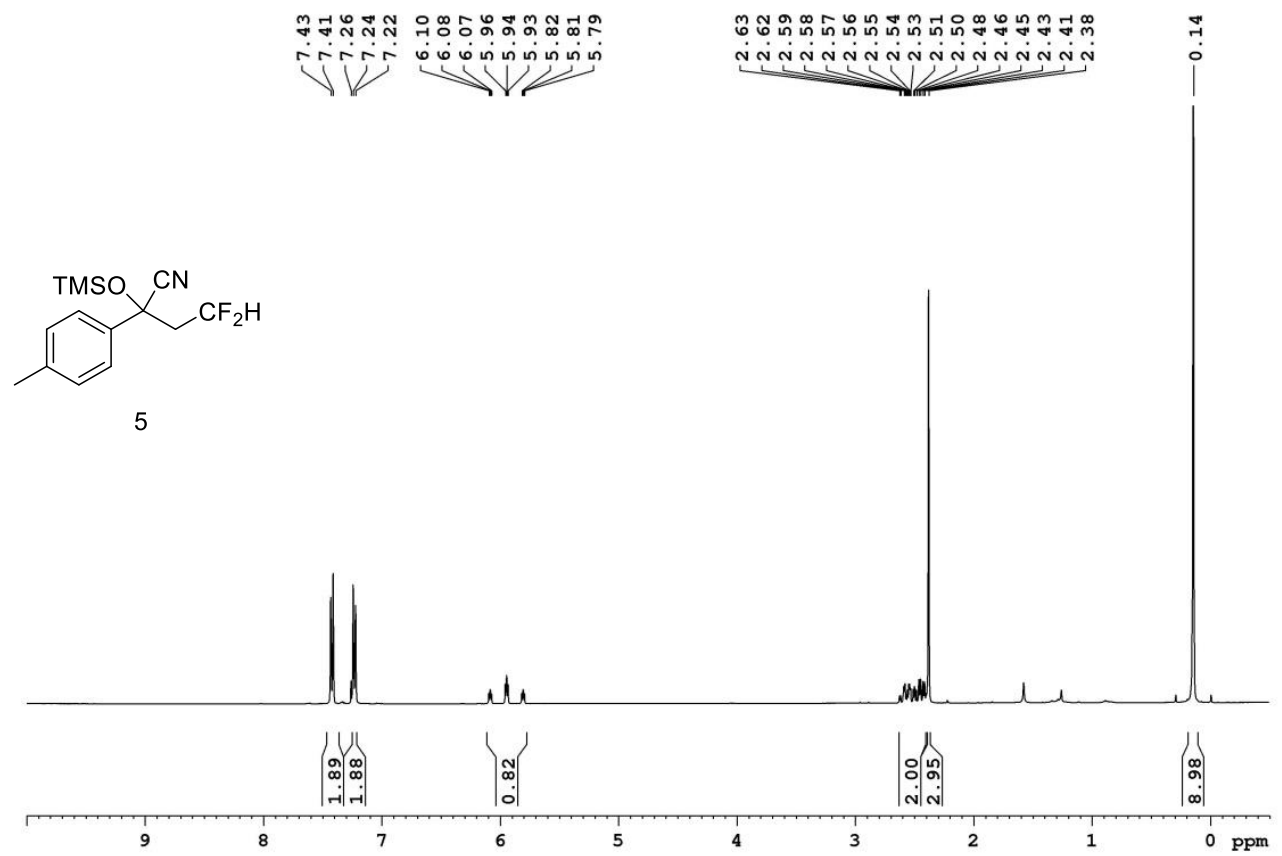

4,4-difluoro-2-(p-tolyl)-2-((trimethylsilyl)oxy)butanenitrile (5): ${ }^{13} \mathrm{C} \mathrm{NMR}\left(100 \mathrm{MHz}, \mathrm{CDCl}_{3}\right)$

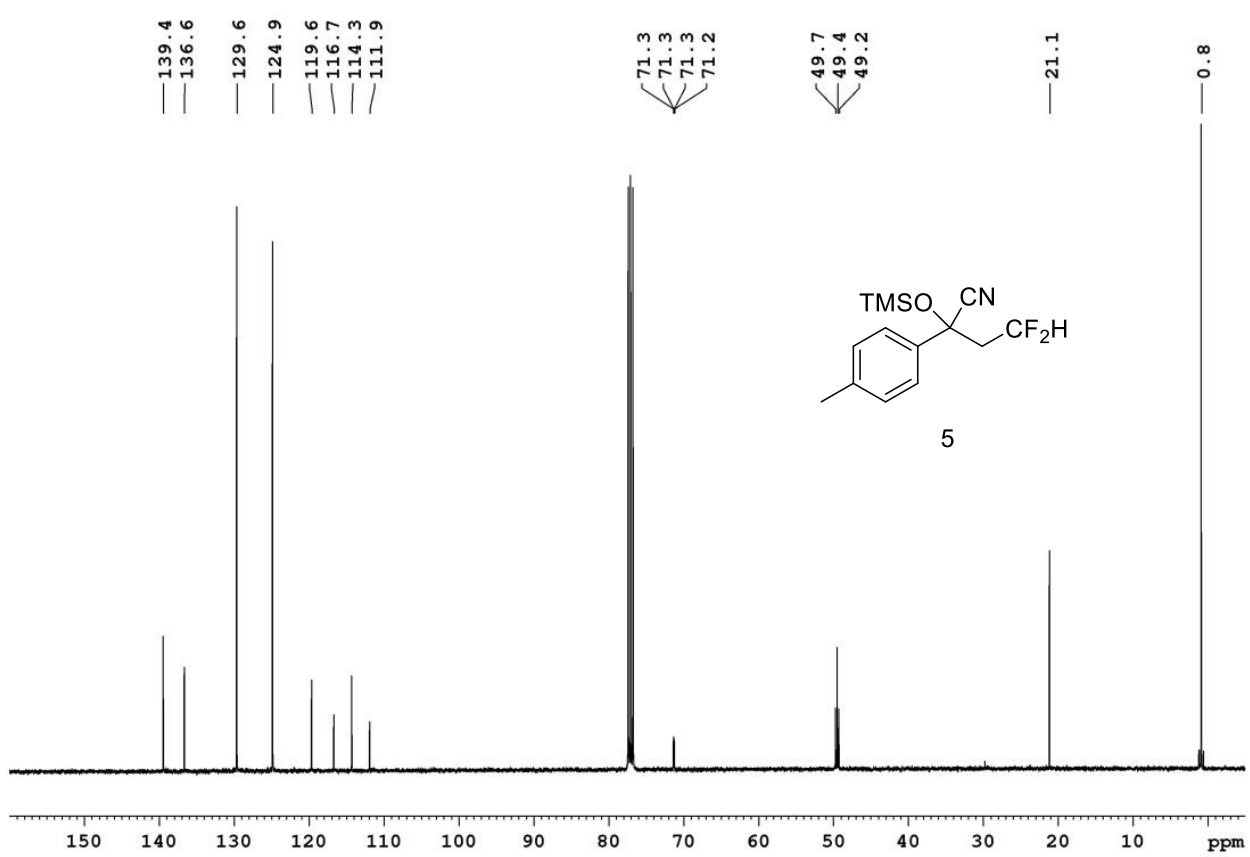



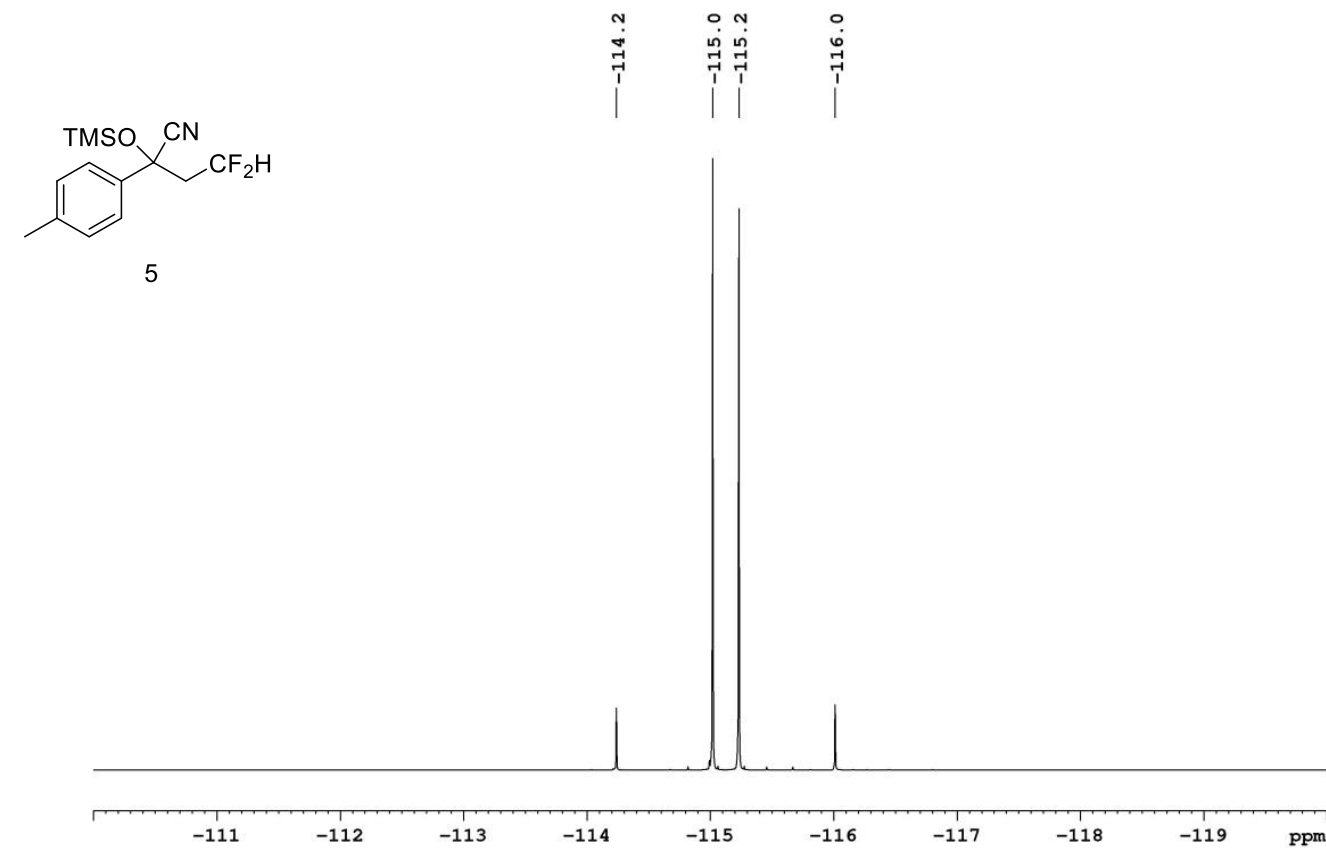

4,4-difluoro-2-(p-tolyl)-2-((trimethylsilyl)oxy)butanenitrile (5): (HRMS)

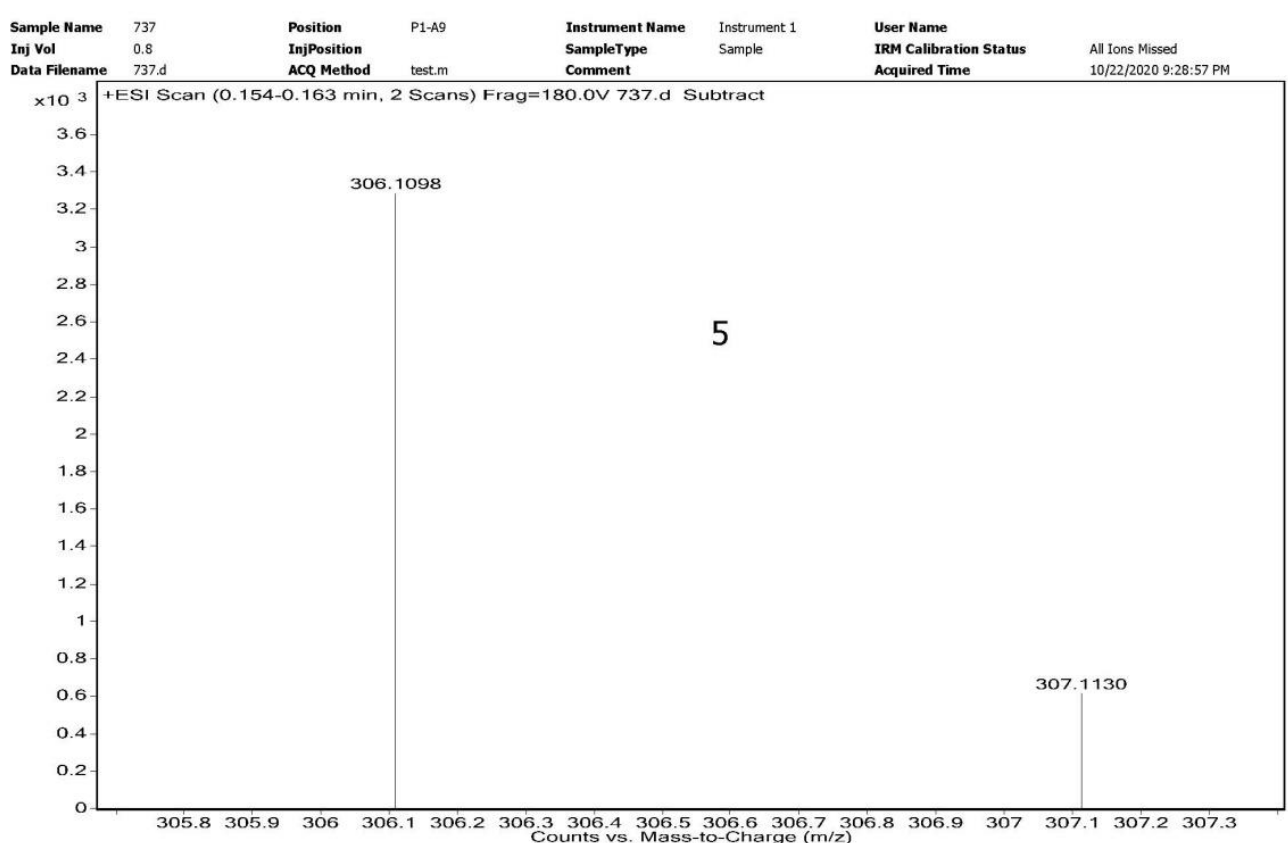


4,4-difluoro-2-(p-tolyl)-2-((trimethylsilyl)oxy)butanamide (6): ${ }^{1} \mathrm{H}$ NMR $\left(400 \mathrm{MHz}, \mathrm{CDCl}_{3}\right)$

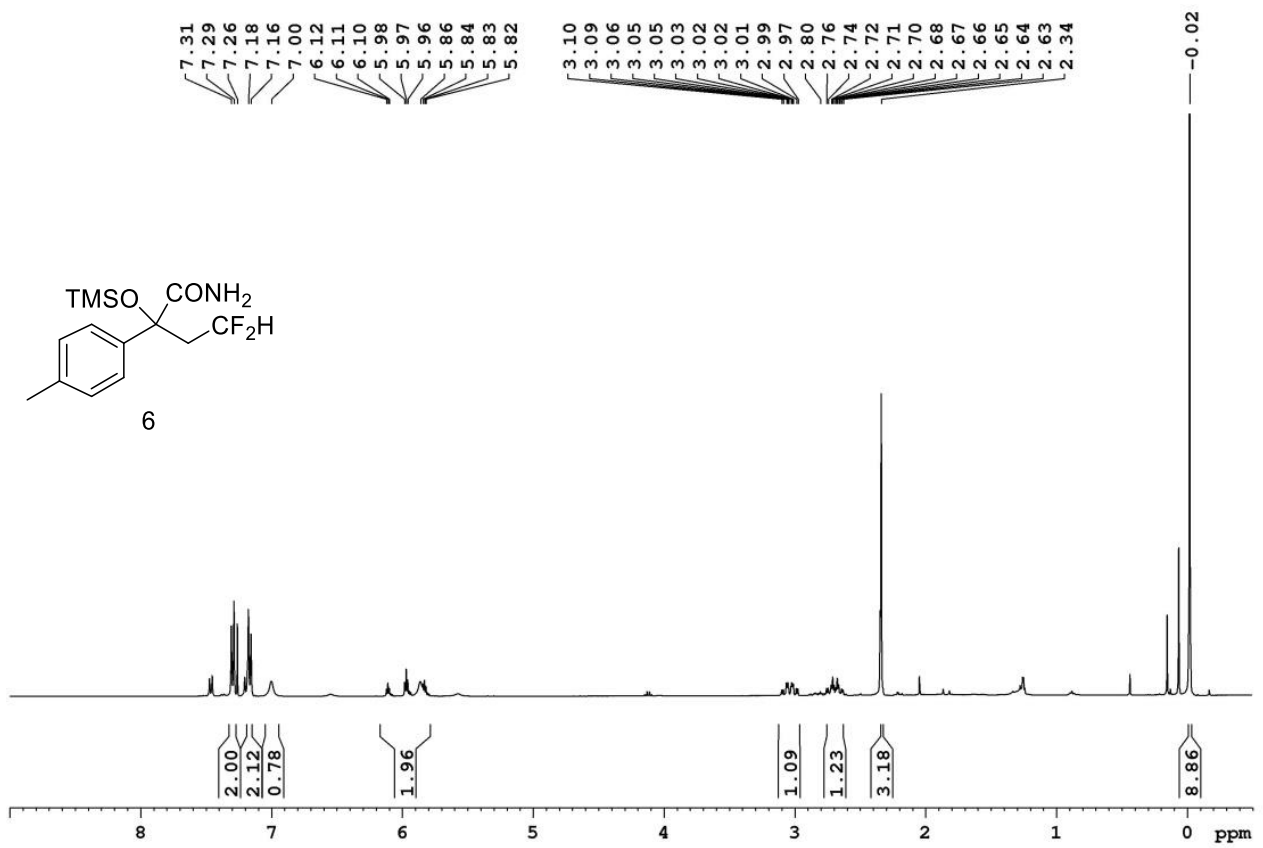

4,4-difluoro-2-(p-tolyl)-2-((trimethylsilyl)oxy)butanamide (6): ${ }^{13} \mathrm{C} \mathrm{NMR}\left(100 \mathrm{MHz}, \mathrm{CDCl}_{3}\right)$

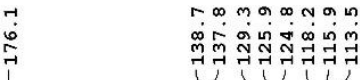

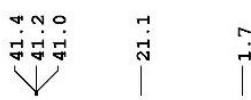

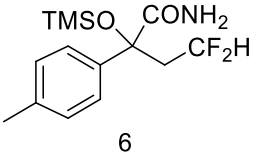

6 
4,4-difluoro-2-(p-tolyl)-2-((trimethylsilyl)oxy)butanamide (6): ${ }^{19} \mathrm{~F}$ NMR (376.5 $\left.\mathrm{MHz}, \mathrm{CDCl}_{3}\right)$

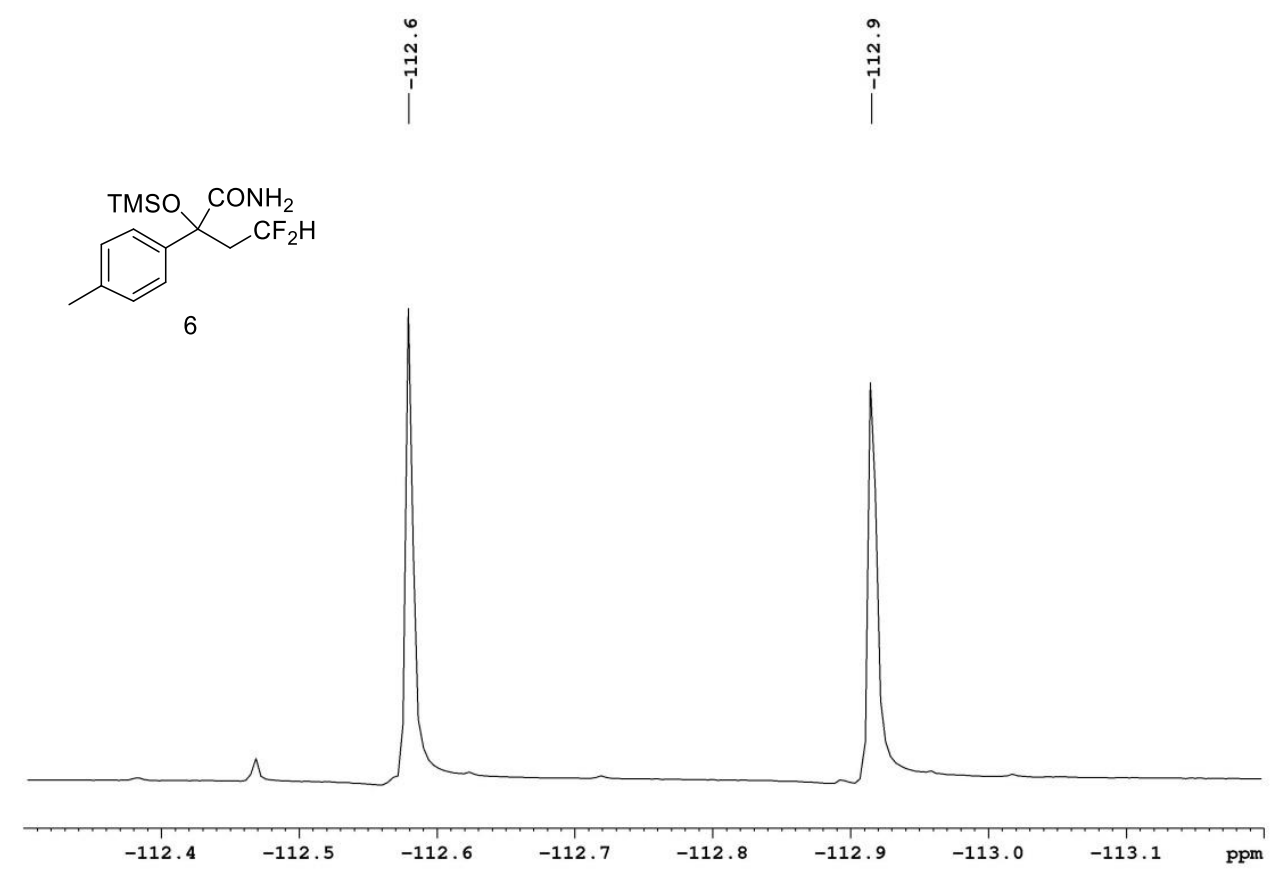

(E)-1-(6-methoxynaphthalen-2-yl)-3-(phenylamino)prop-2-en-1-one (7): ${ }^{1} \mathrm{H}$ NMR (400 MHz, $\left.\mathrm{CDCl}_{3}\right)$

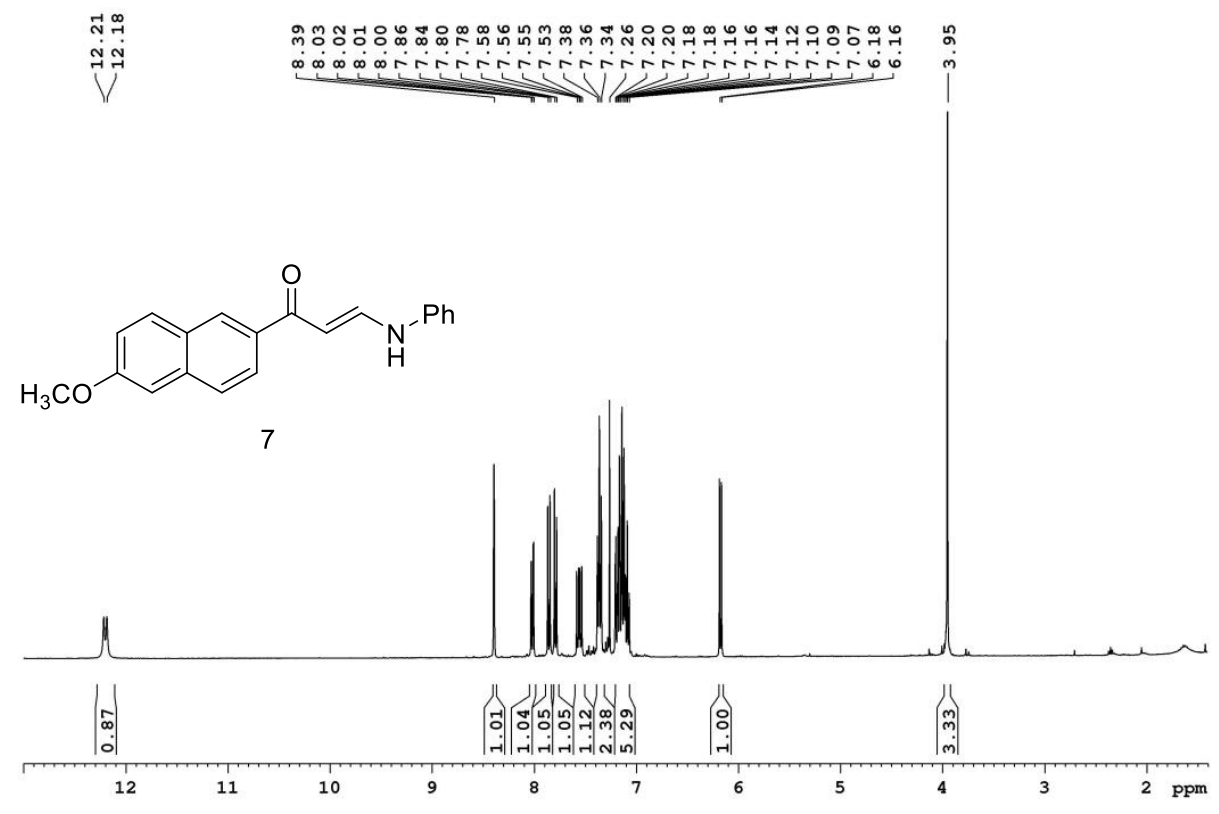


(E)-1-(6-methoxynaphthalen-2-yl)-3-(phenylamino)prop-2-en-1-one (7): ${ }^{13} \mathrm{C}$ NMR (100 MHz, $\left.\mathrm{CDCl}_{3}\right)$

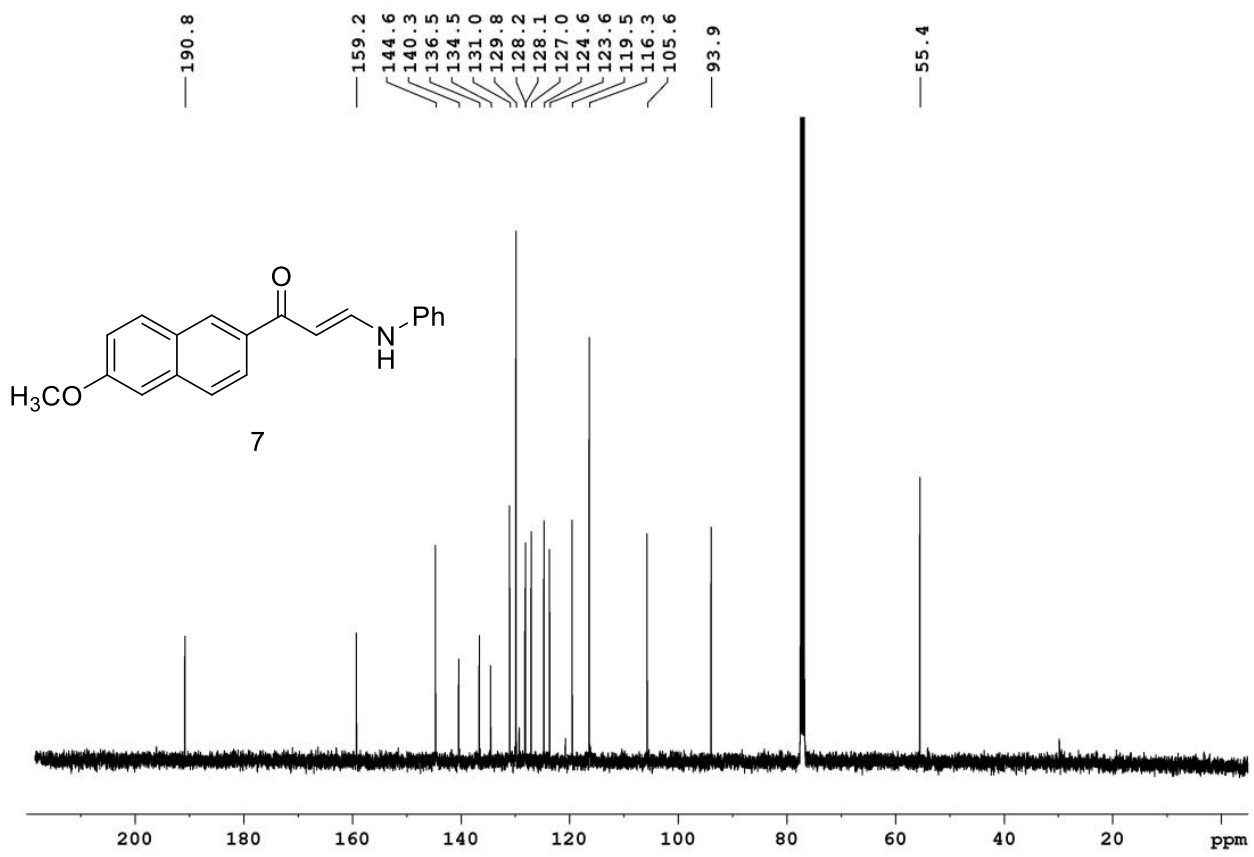

(E)-1-(6-methoxynaphthalen-2-yl)-3-(phenylamino)prop-2-en-1-one (7): HSQC

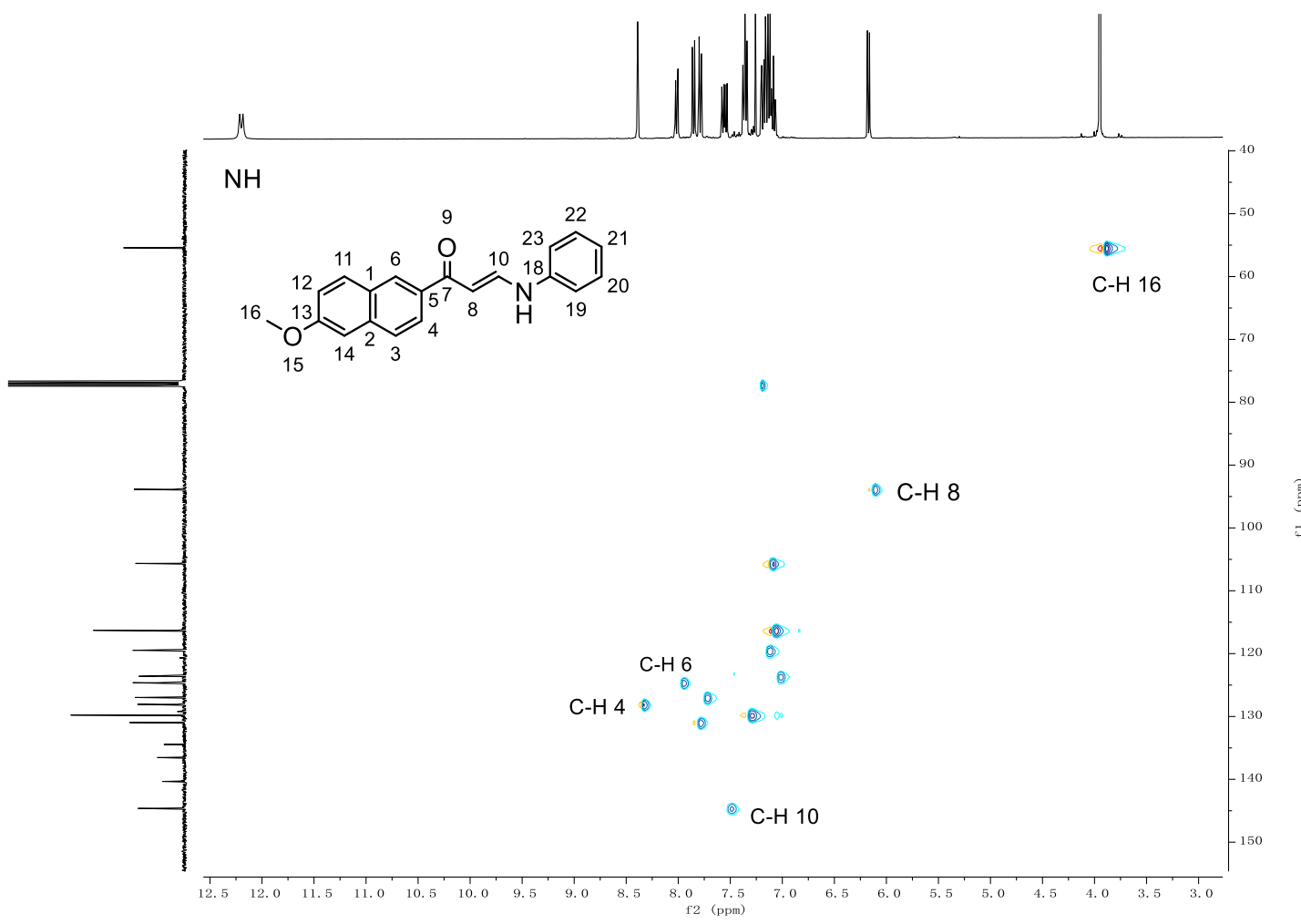


(E)-1-(6-methoxynaphthalen-2-yl)-3-(phenylamino)prop-2-en-1-one (7): ${ }^{1} \mathrm{H}-{ }^{1} \mathrm{H}$ COSY

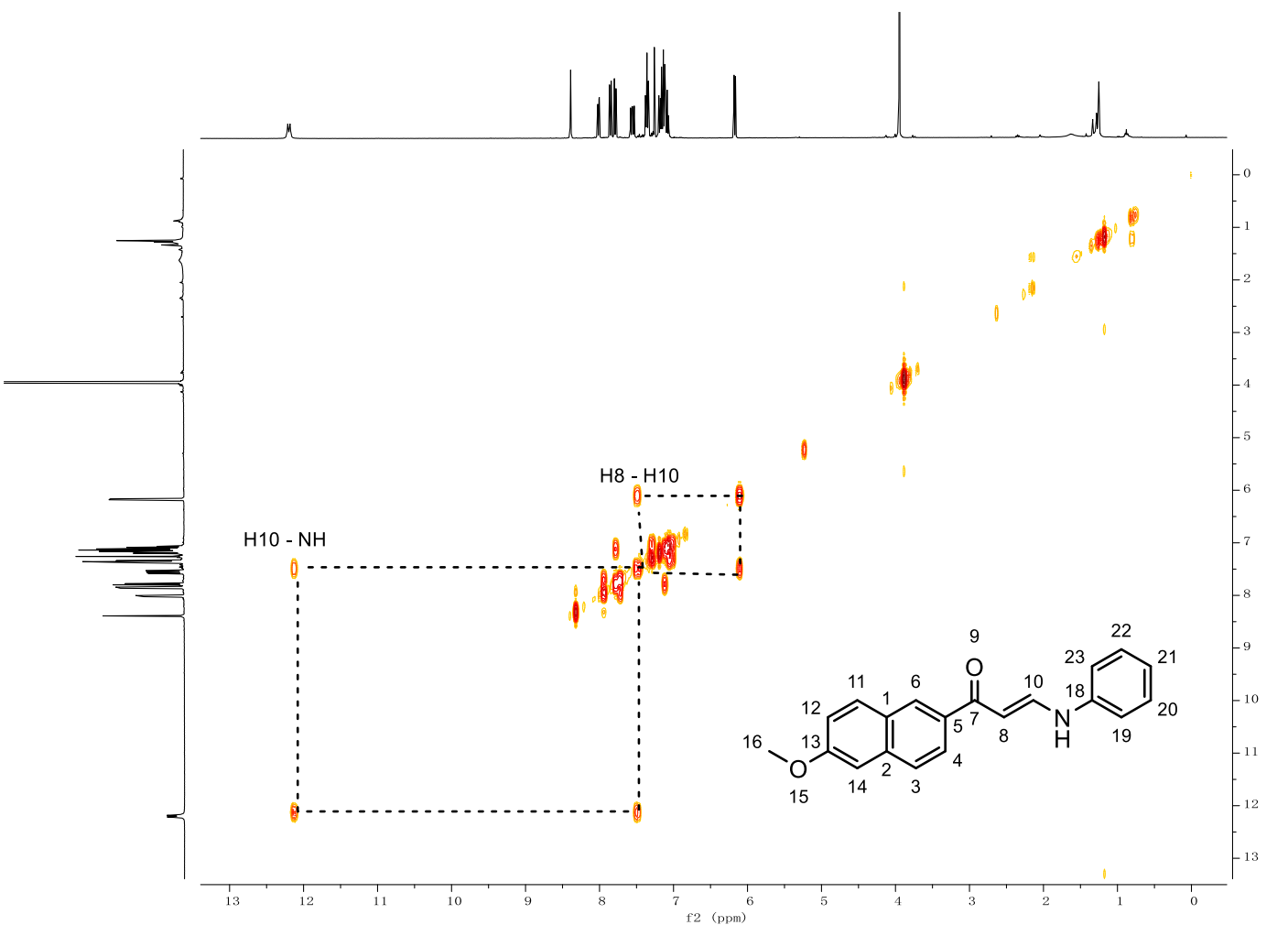

(E)-1-(6-methoxynaphthalen-2-yl)-3-(phenylamino)prop-2-en-1-one (7): NOE

Protons $(\mathrm{H} 8, \mathrm{H10}, \mathrm{NH})$ do not show any NOE effects

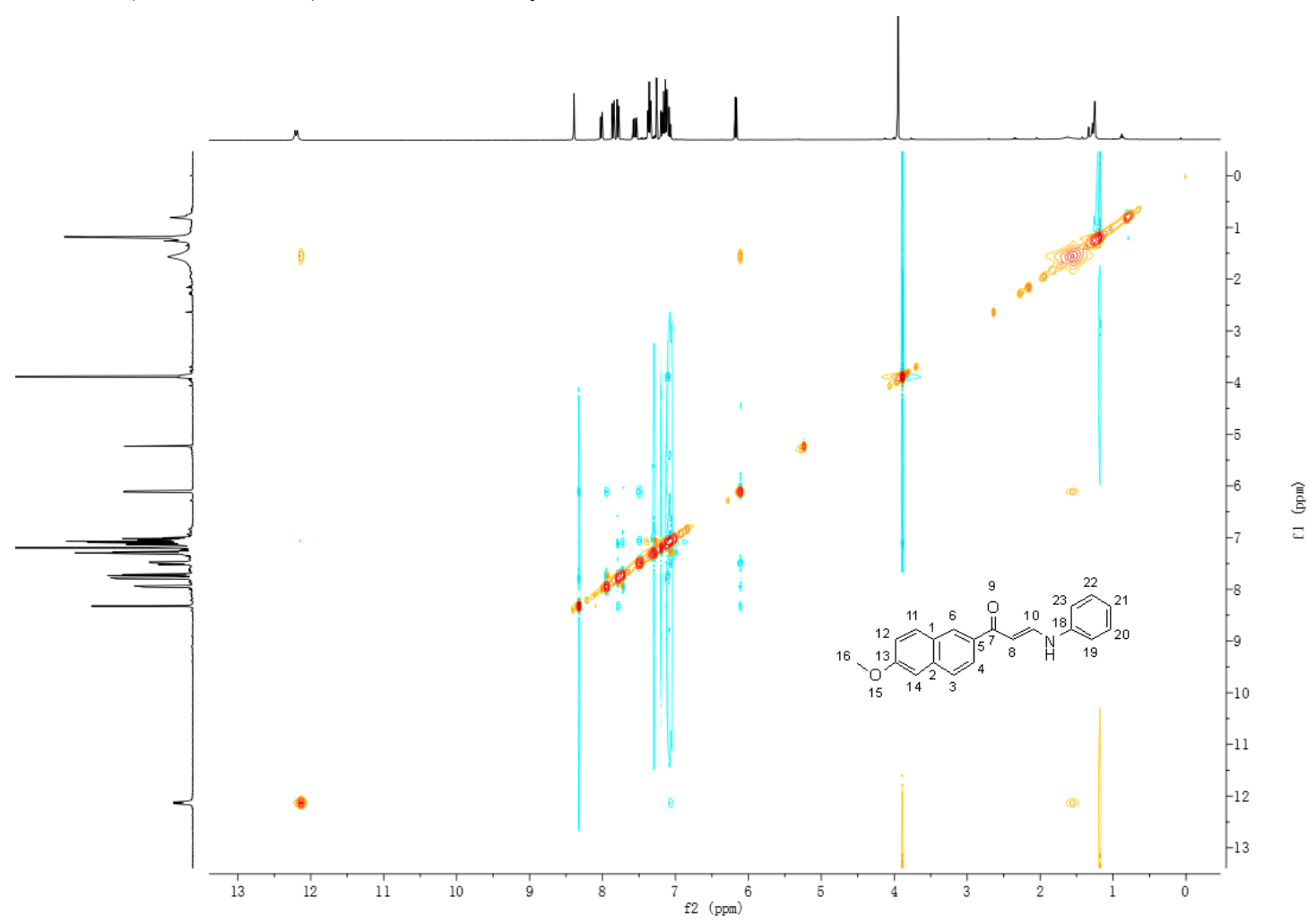


(E)-1-(6-methoxynaphthalen-2-yl)-3-(phenylamino)prop-2-en-1-one (7): HMBC

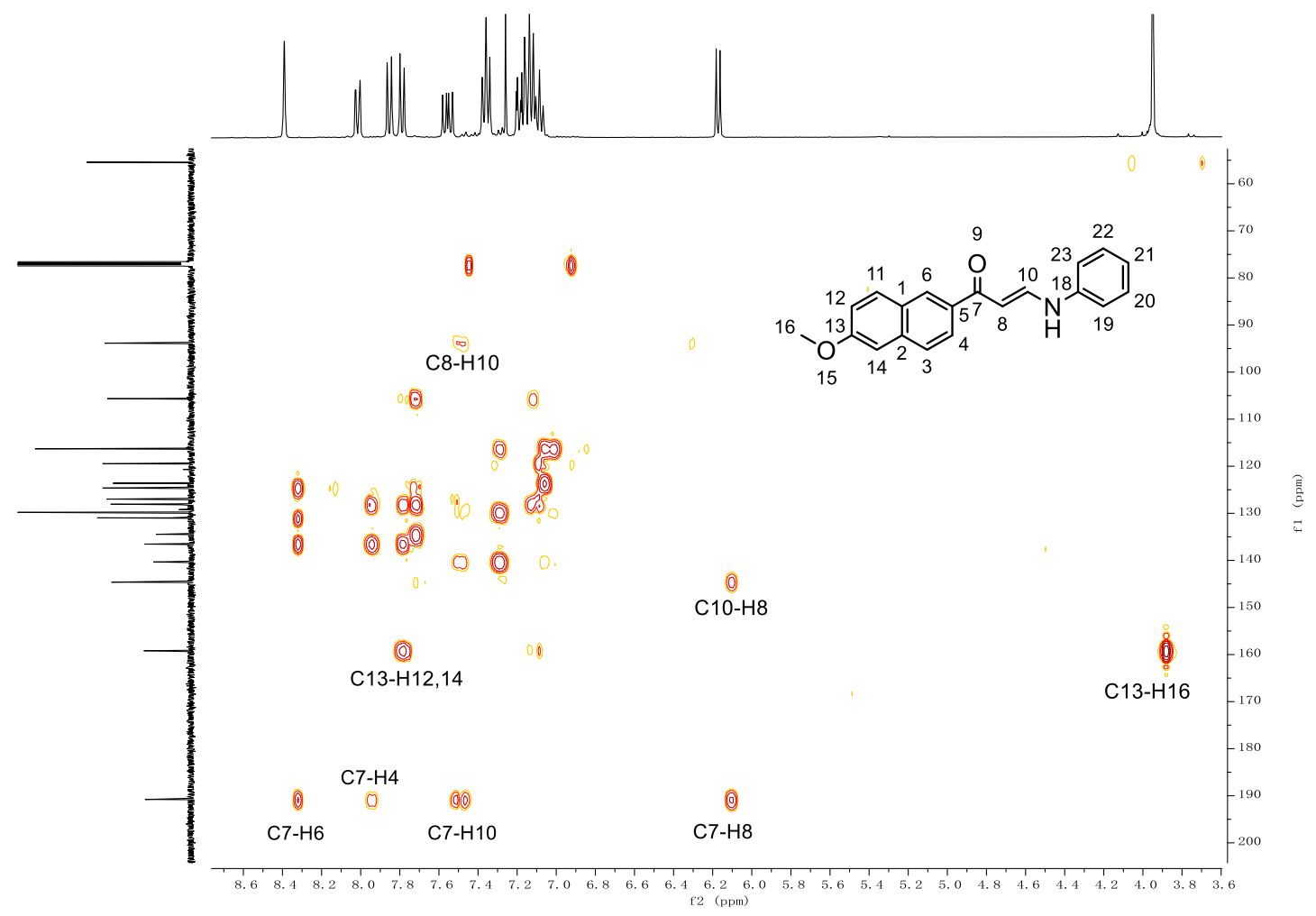

The coupling constant of product 7 is similar to the literature ${ }^{15}$. Based on the spectra and literature, we believe the structure of product 7 is transconfiguration.

(E)-1-(6-methoxynaphthalen-2-yl)-3-(phenylamino)prop-2-en-1-one (7): (HRMS)

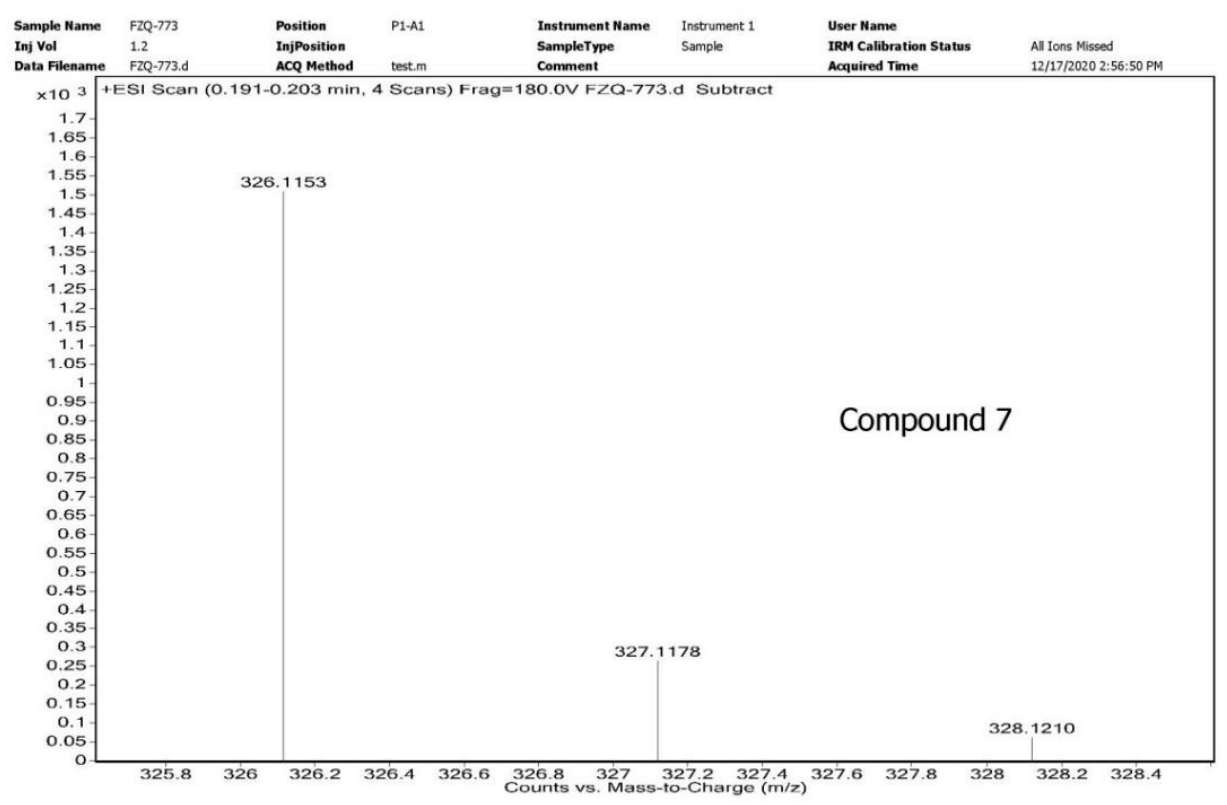




\section{References}

1. Berkessel, A.; Sebastian, L.; Müller, T. N. Lipase/Aluminum-Catalyzed Dynamic Kinetic Resolution of Secondary Alcohols. Angew. Chem. Int. Ed. 2006, 45, 6567-6570.

2. Gärtner, D.; Stein, A. L.; Grupe, S.; Arp, J.; von Wangelin, A. J. Iron-Catalyzed Cross-Coupling of Alkenyl Acetates. Angew. Chem. Int. Ed. 2015, 54, 10545-10549.

3. Yu, J.-Y.; Shimizu, R.; Kuwano, R. Selective Cine Substitution of 1-Arylethenyl Acetates with Arylboron Reagents and a Diene/Rhodium Catalyst. Angew. Chem. Int. Ed. 2010, 49, 6396-6399.

4. Song, C.-X.; Cai, G.-X.; Farrell, T. R.; Jiang, Z.-P.; Li, H.; Gan, L.-B.; Shi, Z.-J. Direct Functionalization of Benzylic C-Hs with Vinyl Acetates via Fe-Catalysis. Chem. Commun. 2009, 45, 6002-6004.

5. Lu, Y.; Li, Y.; Zhang, R.; Jin, K.; Duan, C. Highly efficient Cu(I)-Catalyzed Trifluoromethylation of Aryl(heteroaryl) Enol Acetates with $\mathrm{CF}_{3}$ Radicals Derived from $\mathrm{CF}_{3} \mathrm{SO}_{2} \mathrm{Na}$ and $\mathrm{TBHP}$ at Room Temperature. J. Fluorine Chem. 2014, 161, 128-133.

6. Geibel, I.; Dierks, A.; Müller T., Christoffers, J. Formation of $\delta$-Lactones with anti-BaeyerVilliger Regiochemistry: Investigations into the Mechanism of the Cerium-Catalyzed Aerobic Coupling of $\beta$-Oxoesters with Enol Acetates. Chem. Eur. J. 2017, 23, 7245-7254.

7. Noji, M.; Ohno, T.; Fuji, K.; Futaba, N.; Tajima, H.; Ishii, K. Secondary Benzylation Using Benzyl Alcohols Catalyzed by Lanthanoid, Scandium, and Hafnium Triflate. J. Org. Chem. 2003, $68,9340-9347$.

8. Zheng, J.; Cai, J.; Lin, J.-H.; Guo, Y.; Xiao, J.-C. Synthesis and Decarboxylative Wittig Reaction of Difluoromethylene Phosphobetaine. Chem. Commun. 2013, 49, 7513-7515.

9. Deng, Z.; Lin, J.-H.; Cai, J.; Xiao, J.-C. Direct Nucleophilic Difluoromethylation of Carbonyl Compounds. Org. Lett. 2016, 18, 3206-3209.

10. Tamayo, A. B.; Alleyne, B. D.; Djurovich, P. I.; Lamansky, S.; Tsyba, I.; Ho, N. N.; Bau, R.; Thompson, M. E. Synthesis and Characterization of Facial and Meridional Triscyclometalated Iridium(III) Complexes. J. Am. Chem. Soc. 2003, 125, 7377-7387.

11. Nakayama, Y.; Ando, G.; Abe, M.; Koike, T.; Akita, M. Keto-Difluoromethylation of Aromatic Alkenes by Photoredox Catalysis: Step-Economical Synthesis of $\alpha-\mathrm{CF}_{2} \mathrm{H}-\mathrm{Substituted} \mathrm{Ketones} \mathrm{in}$ Flow. ACS Catal. 2019, 9, 6555-6563.

12. Brown, H. C.; Krishnamurthy, S. Forty Years of Hydride Reductions. Tetrahedron 1979, 35, 567-607.

13. Belletire, J. L.; Howard, H.; Donahue, K. A Facile Synthesis of Phenylacetic Acids from Aryl Ketones. Synth. Commun. 1982, 12, 763-767.

14. Kim, E. S.; Lee, H. S.; Kim, S. H.; Kim, J. N. An Efficient $\mathrm{InCl}_{3}$-Catalyzed Hydration of Nitriles to Amides: Acetaldoxime as an Effective Water Surrogate. Tetrahedron Lett. 2010, 51, 1589-1591.

15. Haak, E. Ruthenium Complexes of Electronically Coupled Cyclopentadienone Ligands-Catalysts for Transformations of Propargyl Alcohols. Eur. J. Org. Chem. 2007, 2815-2824. 